\title{
Standing Waves On An Infinitely Deep Perfect Fluid Under Gravity
}

\author{
G. Iooss* P. I. Plotnikov ${ }^{\dagger}$ J. F. Toland ${ }^{\ddagger}$
}

\begin{abstract}
The existence of two-dimensional standing waves on the surface of an infinitely deep perfect fluid under gravity is established. When formulated as a second order equation for a real-valued function $w$ on the 2-torus and a positive parameter $\mu$, the problem is fully nonlinear (the highest order $x$-derivative appears in the nonlinear term but not in the linearization at 0 ) and completely resonant (there are infinitely many linearly independent eigenmodes of the linearization at 0 for all rational values of the parameter $\mu$ ). Moreover, for any prescribed order of accuracy there exists an explicit approximate solution of the nonlinear problem in the form of a trigonometric polynomial. Using a Nash-Moser method to seek solutions of the nonlinear problem as perturbations of the approximate solutions, the existence question can be reduced to one of estimating the inverses of linearized operators at non-zero points. After changing coordinates these operators become first order and non-local in space and second order in time. After further changes of variables the main parts become diagonal with constant coefficients and the remainder is regularizing, or quasi-one-dimensional in the sense of [22]. The operator can then be inverted for two reasons. First, the explicit formula for the approximate solution means that, restricted to the infinite-dimensional kernel of the linearization at zero, the inverse exists and can be estimated. Second, the small-divisor problems that arise on the complement of this kernel can be overcome by considering only particular parameter values selected according to their Diophantine properties. A parameter-dependent version of the Nash-Moser implicit function theorem now yields the existence of a set of unimodal standing waves on flows of infinite depth, corresponding to a set of values of the parameter $\mu>1$ which is dense at 1 . Unimodal means that the term of smallest order in the amplitude is $\cos x \cos t$, which is one of many eigenfunctions of the completely resonant linearized problem.
\end{abstract}

\footnotetext{
*Institut Universitaire de France, INLN, Université de Nice, 1361 rte des Lucioles, Sophia Antipolis, Valbonne 06560, France.

Gerard.Iooss@inln.cnrs.fr

${ }^{\dagger}$ Russian Academy of Sciences, Lavryentyev pr. 15, Novosibirsk 630090, Russia. plotnikov@hydro.nsc.ru

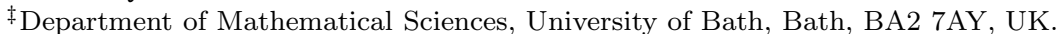
jft@maths.bath.ac.uk
} 


\section{Contents}

1 Introduction 4

1.1 Linear Theory . . . . . . . . . . . . . . . . . . . . 4

1.2 Nonlinear theories .................. 5

1.3 Method .................... 6

1.4 Nonlinear wave equations . . . . . . . . . . . . . . . 8

1.5 Summary ....................... 9

2 Basic Formulation $\quad 10$

3 Notation and Identities $\quad 10$

4 Main Equation $\quad \mathbf{1 3}$

4.1 Linearization at the origin . . . . . . . . . . . . . . . . . . . 14

4.2 Approximate solutions . . . . . . . . . . . . . . . . 17

4.3 An equation for standing waves . . . . . . . . . . . . . 18

5 Linearization at a Non-zero Point $\quad 19$

6 Reduction of Linearized Operator $\mathbf{2 5}$

6.1 Strategy . . . . . . . . . . . . . . . 25

6.2 Change of coordinates and smoothing operators . . . . . . . . . 27

6.3 New linearized equation . . . . . . . . . . . . . . . . 30

7 Normal Form of the Linearized Operator 33

7.1 Simplification of $(6.17) \ldots \ldots . \ldots . \ldots 33$

7.2 The coefficients in $(7.5) \ldots \ldots . \ldots . \ldots . \ldots 37$

7.3 Third change of variable - normalization . . . . . . . . . . 38

8 Inversion of the Linearized Operator $\quad 42$

8.1 The kernel and a compatibility condition . . . . . . . . . . . . . 42

8.2 Principal part of $\mathbb{P} \mathcal{A}^{(0)} \mathbb{P}$ when $\boldsymbol{w}=\boldsymbol{w}_{\boldsymbol{\varepsilon}}^{(\boldsymbol{N})}, \boldsymbol{N} \geq \mathbf{2} \ldots \ldots . . .43$

8.3 Structure of the linear operator . . . . . . . . . . . . . . . . 45

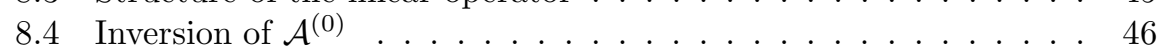

8.5 Inversion of $\Lambda(u, \varepsilon) \ldots \ldots \ldots$. . . . . . . . . . 49

9 Existence of Standing Waves $\quad 51$

A Proof of Lemma 3.1

B Calculation of Approximate Solutions $\quad 56$

$\begin{array}{ll}\text { C Proof of Proposition 5.2 } & 58\end{array}$

D Proof of Proposition 5.3 $\quad 58$

E Proof of Proposition 5.4 60 
F Proof of Lemma 5.1 Concluded $\quad 63$

G Changes of Variables $\quad 63$

H Proofs of Lemmas 6.3, 6.5 and Corollaries 65

I Proof of Lemma 6.8 66

$\begin{array}{lll}\text { J Proof of Corollary 6.10 } & 67\end{array}$

$\begin{array}{ll}\text { K Proof of Lemma } 7.1 & 71\end{array}$

$\begin{array}{lll}\text { L Estimates of } \mathcal{G}_{0} \text { in Lemma } 7.2 & 74\end{array}$

$\begin{array}{ll}\text { M Proof of Theorem 7.5 } & 80\end{array}$

$\begin{array}{ll}\text { N A Nash-Moser Theorem with a Parameter } & 89\end{array}$

$\begin{array}{ll}\text { O Small Divisors and Hypothesis (E) } & 103\end{array}$

O.1 Small divisors . . . . . . . . . . . . . . . . . . . . . . 104

O.2 An extension . . . . . . . . . . . . . . . . 106

O.3 Hypothesis (E) . . . . . . . . . . . . . . . . . . . . 107 


\section{Introduction}

This paper concerns the existence of non-trivial two-dimensional periodic standing waves on water of infinite depth. By a periodic standing wave we mean the two-dimensional motion under gravity of a perfect fluid of infinite depth with a free surface that is periodic in both space and time. The motion beneath the surface is required to be irrotational and, with the position of the free surface determined by the motion of its particles, the pressure there must be a constant, independent of spatial location at all instances of time. We consider only waves with a fixed line of spatial symmetry and motions that are even in time. Such waves are truely time-dependent in the sense that they are not stationary with respect to a moving reference frame. The particles on the surface can be thought of as a continuum of nonlinear oscillators coupled through the motion under gravity of the inviscid fluid below. At time $t$ they form the graph $\{(x, \eta(x, t): x \in \mathbb{R}\}$ of a function which has period $\lambda$ in $x$ and period $T$ in $t$. A fluid at rest filling a half-space beneath any horizontal line is a trivial solution of this classical problem. The conditions of symmetry in space and time adopted here simplify the mathematical formulation by removing degeneracies due to translational invariance in $x$ and $t$; they are not an intrinsic part of the standing-wave problem and one could imagine more general solutions, for example, 'travelling-standing-wave' solutions, of the free boundary problem. The standing-wave problem may also be formulated for flows with finite depth, where the water is contained above an impermeable horizontal bottom and, as we will see, the technical issues involved there are somewhat different.

\subsection{Linear Theory}

When the standing water-wave problem is linearized about a trivial solution, we find a linear boundary-value problem, first derived satisfactorily by Poisson in 1818, although Laplace in 1776 came very close (see [10]):

$$
\begin{aligned}
\frac{\partial^{2} \phi}{\partial x^{2}}+\frac{\partial^{2} \phi}{\partial y^{2}} & =0, & & x, t \in \mathbb{R}, \quad y<0, \\
\phi(x+\lambda, y, t) & =\phi(x, y, t)=\phi(x, y, t+T), & & x, t \in \mathbb{R}, \quad y<0, \\
\phi(-x, y, t) & =\phi(x, y, t)=-\phi(x, y,-t), & & x, t \in \mathbb{R}, \quad y<0, \\
\nabla \phi(x, y, t) & \rightarrow(0,0), & & y \rightarrow-\infty, \\
\frac{\partial^{2} \phi}{\partial t^{2}}+g \frac{\partial \phi}{\partial y} & =0, & & y=0 .
\end{aligned}
$$

The acceleration due to gravity is denoted by $g, \phi$ is the velocity potential of a 'linear standing wave' and $\eta$, the linear wave elevation, is given by $g \eta(x, t)=$ $-(\partial \phi / \partial t)(x, 0, t)$. When $\mu=g T^{2} / 2 \pi \lambda$ is irrational there are no solutions except for constants. But, when $\mu$ is rational, this problem is highly degenerate because every function of the form

$$
\phi(x, y, t)=\sin \left(\frac{2 n \pi t}{T}\right) \cos \left(\frac{2 m \pi x}{\lambda}\right) \exp \left(\frac{2 m \pi y}{\lambda}\right) \text { with } \frac{n^{2}}{m}=\mu
$$


is a solution of (1.1). Thus the set of 'eigenvalues' $\mu$ of the linearized problem is $\mathbb{Q}^{+}$, which is dense in $[0, \infty)$, and each eigenvalue has an infinite set of linearly independent eigenfunctions. We refer to this latter property as complete resonance, by analogy with the theory of nonlinear oscillators. In the case of finite depth the boundary condition (1.1d) must be replaced by

$$
\frac{\partial \phi}{\partial y}(x,-h, t)=0, \quad x, t \in \mathbb{R} .
$$

Then linear eigenvalues can have unique normalized eigenfunctions. In both cases the linearized problem can be re-formulated as an equation of the form

$$
u_{t t}+\mu A u=0,
$$

where $A$ is non-negative, constant-coefficients, self-adjoint non-local pseudodifferential operator acting on functions defined on $S^{1}, u$ maps the torus $\mathbb{T}^{2}$ into $\mathbb{R}$ and $\mu$ is a positive parameter. For both finite and infinite depth, the nonlinear existence theory is made difficult because of small-divisor problems that arise when linear operators of hyperbolic type, similar to (1.2) but with non-constant coefficients, are to be inverted at each step of the iteration. (Small divisor problems occur naturally in questions about periodic solutions of hyperbolic equations.) The infinite depth case which we deal with here has the combined problem of complete resonance and small divisors.

\subsection{Nonlinear theories}

In 1847 Stokes gave a nonlinear theory of travelling waves [29]. It was, we believe, Boussinesq in 1877 who was the first to deal with nonlinear standingwaves. On pages 332-335 and 348-353 of [7] he refers to 'le clapotis', meaning standing waves, and his treatment, which includes the cases of finite and infinite depth, is a nonlinear theory taken to second order in the amplitude. Seemingly unaware of this work, Lord Rayleigh [26] developed a third order theory that included travelling and standing waves on infinite depth as special cases. (Much later Tadjbaksh \& Keller [31] used a different expansion to obtain a third-order theory in the case of finite depth.) These theories deal with Eulerian descriptions of the flow. In 1947 Sekerkh-Zenkovich took the theory to fourth order using Lagrangian coordinates. (This paper cites works by E. Guyou and by M. Larras, but says that they are not rigorous. It is our view also that they are flawed.) In 1952 Penney \& Price took up the question of a highest standing wave (in 1880 Stokes had conjectured the existence of a highest travelling wave [30]) using a fifth-order nonlinear theory. Although he was motivated by suspicions about the basis of their theory, Taylor [32] reported on experiments which served to confirm the conclusions of Penney \& Price [21], and Okamura [20] has recently extended numerically these investigations. See Wehausen [35, page 587 and the references therein] for a literature survey up to 1965. Like Boussinesq, he refers to standing waves as 'clapotis'. In 1981, Schwartz \& Whitney considered the full nonlinear problem in Eulerian coordinates and, using conformal mappings, 
developed an algorithm for calculating the coefficients in a formal power-series expansion of a standing wave to any order, provided that a resonance problem did not occur in executing the $n^{2}$ step in the algorithm, for any integer $n$. Extensive numerical investigations in which they successfully calculated standing waves to order twenty-five convinced them of the validity of their approach. A proof [1] that problems with resonances never arise confirmed that the algorithm always gives a formal power series solution of the standing-wave problem on infinite depth. The question of convergence of this power series remained open, but the observation, (subsequently generalized in [13, 14]) plays a significant rôle in our existence theory. Here our treatment of the nonlinear problem on infinite depth is based on a formulation derived from a general Hamiltonian description of two-dimensional wave motion due to Dyachenko, Kuznetsov, Spector \& Zakharov [11] (see also [36] and [4, Appendix]). The standing-wave form of the theory is discussed in detail in [16] and recalled here in Section 2. This formulation is neither Eulerian nor Lagrangian, since the dependent variables are the $y$-coordinate (the wave height) and the velocity potential at the surface. A complete description of the underlying flow, in Eulerian or Lagrangian variables, can be inferred once a solution of the Hamiltonian system has been found. One obvious advantage of this formulation is that operators in the equation appear explicitly. In Lagrangian variables the nonlinear operator which gives $A$ in (1.2) as its linearization is a non-local Dirichlet-Neumann operator defined implicitly by an unknown domain and it is painful to contemplate the intricate calculations that must be taken to high order because of complete resonance in the infinite-depth problem. However a Lagrangian description was used in a successful proof [22] of the existence of standing waves, for certain values of the physical parameters in the problem of finite depth, when complete resonance is absent.

\subsection{Method}

We do not yet know how to exploit the Hamiltonian structure of (K) and (D) in Section 2 directly. So we adopt a 'partial-differential-equations' methods and, by eliminating the velocity-potential variable, reduce the system to a nonlinear integro-differential equation equation (4.1) for a periodic, real-valued function of two real variables, with a view to applying a fixed-point argument for existence. (In doing so we lose the variational structure.) To explain the proof of existence, suppose that the equation for standing waves is

$$
\mathcal{F}(w, \mu)=0, \quad w \text { not a constant, }
$$

where $w$ is in a neighbourhood of 0 in some Banach space $(X,\|\cdot\|), \mu>1$ is a real parameter and $\mathcal{F}(\cdot, \mu):(X,\|\cdot\|) \rightarrow(Y,|\cdot|)$. The actual expression for $\mathcal{F}$ is necessarily rather complicated and we will not identify $X$ and $Y$ at this stage. We expect to need the Nash-Moser method, an abstract version of a rapid-convergence iteration method proposed in $[2,19]$ for existence, because small-divisor problems are known to arise in a study of linear problems of the 
form (1.2). It has been realised for some time $[1,13,14,27]$ that there exists a formal power series solution of the standing problem (see Section 4.2) which gives approximate solutions $(w, \mu)=\left(w_{\varepsilon}^{(N)}, 1+\varepsilon^{2}\right)$ of $(1.3)$ :

$$
\left|\mathcal{F}\left(w_{\varepsilon}^{(N)}, 1+\varepsilon^{2}\right)\right| \leq \text { const }|\varepsilon|^{N+1}, \quad\left\|w_{\varepsilon}^{(N)}\right\| \leq \text { const } \varepsilon .
$$

By seeking solutions of (1.3) for $\varepsilon>0$ as perturbations $u$ of $w_{\varepsilon}^{(N)}$, for any prescribed order of accuracy, we obtain (see Section 4.3) an equation

$$
\Phi(u, \varepsilon)=0
$$

with an explicit closed-form approximate solution. More precisely, for $N \in \mathbb{N}$ with $N \geq 2$, equation (1.4) has an approximate solution $u=u_{\varepsilon}^{(N)}$, which can be expressed explicitly in terms of trigonometric functions and, for a constant in suitable Banach spaces,

$$
\left\|u_{\varepsilon}^{(N)}\right\| \leq \operatorname{const} \varepsilon, \quad\left|\Phi\left(\varepsilon, u_{\varepsilon}^{(N)}\right)\right| \leq \text { const }|\varepsilon|^{N+1} .
$$

It is known from $[13,14]$ that the complete resonance of the linear problem leads, for any fixed order $N>2$, to an infinite set of possible approximate solutions of the nonlinear problem. So we specialize our study by focusing on 'one-mode' solutions, by which we mean solutions that have only one mode, namely $\cos x \cos t$, in their leading term of order $\varepsilon$. Then choosing $N>2$ appears to be sufficient to solve the degeneracy of the linearized problem (and de facto eliminates the other possible solutions). The first step in a proof of existence is in Section 5, where the linearization $\Phi_{u}^{\prime}(u, \varepsilon)$ of $\Phi$ at an arbitrary point $(u, \varepsilon)$ is calculated and simplified. In Section 6 we show that $\Phi_{u}^{\prime}(u, \varepsilon)$ can be decomposed as a sum of linear operators

$$
\Phi_{u}^{\prime}(u, \varepsilon)=\Lambda(u, \varepsilon)+\Gamma(u, \varepsilon) \text { where } \Phi(u, \varepsilon)=0 \text { implies that } \Gamma(u, \varepsilon)=0 .
$$

To apply the version of the Mash-Moser Theorem in Appendix $\mathrm{N}$ it is sufficient to prove that $\Lambda(u, \varepsilon)$ is invertible and to estimate its inverse. At this stage we encounter two difficulties. The first is that $\Lambda$ is a rather complicated linear operator of hyperbolic type with non-constant coefficients (see equations (6.3) and (6.8)) and it is impossible to say straight away whether it is invertible, with suitable estimates, or not. In the remainder of Section 6 , a change of coordinates is introduced to eliminate the partial derivatives $\partial^{2} / \partial x \partial t$ and $\partial^{2} / \partial x^{2}$ in the expression for $\Lambda$. Then, in Section 7, a further change of coordinates is used to show that inverting $\Lambda$ is equivalent to inverting an operator (for now also called $\Lambda$ ) which is the sum of a 'main part' and a 'remainder', where the main part is of the type (1.2) with constant coefficients and the remainder is from the class of Q1D operators introduced in [22] for a similar purpose when dealing with the finite-depth problem. The constant coefficients in the main part are functions of the point of linearization. If the main part can be inverted, then a method of descent [22, Section 9] can be adapted to show that similar estimates hold when inverting the full operator $\Lambda(u, \varepsilon)$ (see Section 8). To calculate an 
inverse of the main part we first need to invert its restriction to the kernel of the linearization about zero, which is infinite-dimensional because of complete resonance. This needs precise and very explicit calculations, which here are humanly possible thanks to the choice of formulation (see the remark in the last paragraph of Section 1.2). Then inverting the main part on the complement of the kernel leads to small-divisor problems and produces estimates of the inverse which depend on Diophantine properties of the coefficients. The outcome is that our estimates of inverses depend on the point of linearization in a highly unstable way. Appendix $\mathrm{N}$ is devoted to a version of the Nash-Moser method in an abstract setting which was developed with these difficulties in mind. It deals the convergence of sequences of parametrized families of approximate solutions to a parametrized family of solutions of (1.4), rather than with the more usual convergence to solutions of sequences of approximate solutions. The domains of the parametrizations shrinks with each iteration, but their intersection is shown to be a non-trivial set upon which convergence to solutions occurs. Starting with parametrized family $\nu_{0}(\varepsilon), \varepsilon \in\left[0, \varepsilon_{0}\right]$, of approximate solutions of (1.4) in the sense of (1.5), the first step is to create a new parametrized family $\nu_{1}(\varepsilon)$, $\varepsilon \in \mathcal{E}_{1}$ of approximate solutions, where $\mathcal{E}_{1}$ is the subset of $\left[0, \varepsilon_{0}\right]$ at which the Diophantine properties of coefficients lead to useful estimates on the inverses of the operators $\Lambda\left(\nu_{0}(\varepsilon), \varepsilon\right)$. The next step is to do this again, creating a new family of approximate solutions $\nu_{2}(\varepsilon), \varepsilon \in \mathcal{E}_{2} \subset \mathcal{E}_{1}$, and so on. For this method to have any hope of yielding the existence of a solution of (1.4), it is obviously necessary that the set $\cap_{k \in \mathbb{N}} \mathcal{E}_{k}$ should have 0 as a limit point. This property is one of the abstract hypothesis of the Nash-Moser theorem (Theorem N.2) which leads to an iteration that converges to a solution of (1.4). Having adopted this strategy, the main part of the paper is devoted to the long calculations needed to verify the hypotheses of Theorem N.2 which yields the existence of standing waves for a set of parameter values $\mu$ with $1<\mu=g T^{2} / 2 \pi \lambda$ ( $T$ is the temporal period, $\lambda$ is the spatial period and $g$ is the acceleration due to gravity) which contains 1 in its closure. Note that we do not establish the existence of standing waves for all $\mu>1$ close to 1 , and the significance of the formal power series found by Schwartz \& Whitney remains uncertain. All that we know for sure is that for all values of $\mu>1$ in a set $E$ which is dense at 1 , there are solutions of the infinite-depth standing-wave problem close to the approximate solutions given by the Schwartz \& Whitney algorithm. (Here $E$ is dense at 1 means that $\lim _{r \backslash 0} r^{-1}$ meas $(E \cap[1,1+r])=1$.) Our main result on the existence of standing waves on deep water is stated precisely in Theorem 9.1, and a consequence due to scaling is explained in Remark 9.2.

\subsection{Nonlinear wave equations}

Rabinowitz's classical paper [25] led to a huge explosion of interest in the global variational theory of semilinear wave equations, and much of the early work is nicely surveyed by Brezis [8], who simplifies many proofs and gives extensive references to the original literature. But the standing-wave problem $(\mathrm{K})$ and (D) of Section 2 does not fit into such abstract theory for a variety of reasons 
and there is currently no global theory of standing waves. First it is not a partial differential equation. Even when linearised it has the form

$$
\ddot{w}-\mu \mathcal{H} w^{\prime}=0
$$

where $\mathcal{H}$ is the Hilbert transform on $2 \pi$-periodic functions and $w \mapsto-\mathcal{H} w^{\prime}$ is non-negative and self-adjoint. Second and more important, it is quasi-linear, the nonlinear term is non-local, and when reduced to a single equation in Section 4 the nonlinear term involves $w^{\prime \prime}$, the highest $x$ derivative, which does not appear in the linear term. If, as in this paper, attention is restricted to the question of bifurcation from the trivial solution we find that the preceding observations make it difficult to see how to apply recent local variational methods, such as those developed by Berti \& Bolle [5, 6] for problems with complete resonance. Also the local theory of Bambusi \& Paleari [3], which is closer in spirit to our approach, is for semi-linear problems. Close also to the spirit of the present work is that of Craig \& Wayne [9] and Wayne [33], which are nicely explained in $[34]$.

\subsection{Summary}

Since the analysis which follows involves some delicate changes of variables with consequent elaborate calculations and many estimates, it is worth bearing in mind the robust features of this approach to the standing-wave problem that lead to a satisfactory outcome.

1. An approximate solution is known in closed form to any required order of accuracy.

2. The Nash-Moser theorem used

(a) requires that only a part of the linearization of the operator equation needs to be inverted at each step, provided the remaining part vanishes at solutions of the equation;

(b) seeks convergence of parametrized families of approximate solutions to parametrized families of solutions.

3. The problem linearized at an arbitrary point can be reduced, by change of variables, to one which is a sum of a main part and a quasi-one-dimensional (Q1D) operator.

4. The method of descent and Q1D theory [22] means that inverting the main part is what matters.

5. The main part can be inverted in two steps:

(a) first, the restriction to the kernel of the linearization at zero can be inverted because we have a good grasp of the form of the operators involved; 
(b) second, the small divisors problem on the complementary space can be dealt with by analysing the size of the parameter set where elementary Diophantine analysis leads to estimates that ensure that the Nash-Moser theory applies.

Acknowledgements. The results of this paper were announced in [15]. John Toland acknowledges the warm hospitality of INLN, Université de Nice, where this work was begun and all three authors acknowledge the warm hospitality of Professor Mariolina Padula during a visit to Dipartimento di Matematica, University of Ferrara, where the work continued with many very fruitful discussions.

\section{Basic Formulation}

Following [11], the standing-wave problem is formulated in [16] as a system of two equations for functions $w$ and $\varphi$, and a positive parameter $\mu$ :

$$
\begin{gathered}
\mathcal{H} \varphi^{\prime}+w^{\prime} \mathcal{H} \dot{w}-\dot{w}\left(1+\mathcal{H} w^{\prime}\right)=0, \\
\mathcal{H}\left(w^{\prime} \dot{\varphi}-\dot{w} \varphi^{\prime}-\mu w w^{\prime}\right)+(\dot{\varphi}-\mu w)\left(1+\mathcal{H} w^{\prime}\right)-\varphi^{\prime} \mathcal{H} \dot{w}=0,
\end{gathered}
$$

where dot means the partial time $(t)$ derivative and prime means a partial space $(x)$ derivative. Both $w$ and $\varphi$ are functions of $(x, t)$ which are even and $2 \pi$-periodic in $x$, and $w$ and $\dot{\varphi}$ are even and $2 \pi$-periodic in $t$ as well. Here $\mathcal{H}$ is the $2 \pi$-periodic Hilbert transform, with respect to $x$, which is defined for locally square integrable functions by

$$
\begin{aligned}
& \mathcal{H}(\cos n x)=-\sin |n| x, \quad \mathcal{H}(1)=0, \\
& \mathcal{H}(\sin n x)=\operatorname{sgn}(n) \cos n x \quad(n \neq 0),
\end{aligned}
$$

A solution of these equations leads to the free surface of a standing wave given parametrically in physical coordinates $(\xi, \eta)$ by

$$
(\xi, \eta)=(x+\mathcal{H} w(x, t),-w(x, t)), \quad(x, t) \in \mathbb{R}^{2},
$$

where $\varphi(x, t)$ is the value of the velocity potential on the free surface and $\mu$, the bifurcation parameter, is

$$
\mu=\frac{g T^{2}}{2 \pi \lambda}
$$

where $T$ is the time period, $\lambda$ is the spatial period, and $g$ is the acceleration due to gravity. It is shown in [16] that the formulation (K) and (D) is equivalent to the classical standing-wave formulation, see, for example, [1].

\section{$3 \quad$ Notation and Identities}

We begin with the two basic function spaces

$$
L_{\natural}^{2}=L^{2}(\mathbb{R} / 2 \pi \mathbb{Z}) \text { and } L_{\text {吼 }}^{2}=L^{2}\left\{(\mathbb{R} / 2 \pi \mathbb{Z})^{2}\right\},
$$


with scalar products $\langle\cdot, \cdot\rangle$, and $\langle\langle\cdot, \cdot\rangle\rangle$, respectively. Let $H_{\natural}^{1}$ and $H_{\natural}^{1}$ denote, respectively, the spaces of functions which, with the components of their gradients, are in $L_{\natural}^{2}$ or in $L_{\text {घต }}^{2}$. Note that $\mathcal{H}$ is a bounded linear operator on $L_{\natural}^{2}$ with

$$
\mathcal{H}^{*}=-\mathcal{H},
$$

where $\mathcal{H}^{*}$ denotes the adjoint of $\mathcal{H}$. In fact, for $v \in L_{\natural}^{2}, \mathcal{H}$ is given by the Cauchy principle value integral

$$
\mathcal{H} v(x)=-\frac{p \cdot v \cdot}{2 \pi} \int_{-\pi}^{\pi} \frac{v(s) d s}{\tan \frac{1}{2}(x-s)}
$$

which exists almost everywhere if $v$ is merely integrable. (However, while it is defined almost everywhere, $\mathcal{H} v$ is not necessarily integrable in this case.) This formula leads to Privalov's Theorem [37] which implies that $\mathcal{H}$ is continuous from the space $C^{k, \alpha}$ of $2 \pi$-periodic functions which, with their first $k$ derivatives, are Hölder continuous with exponent $\alpha$, into itself. In particular, the Hilbert transform of a smooth function is smooth. For $f$ and $g \in L_{\natural}^{2}$ or $L_{\text {吅 }}^{2}$ let

$$
\begin{gathered}
{[f, g]=f \mathcal{H} g-g \mathcal{H} f \text { and } J(f, g)=f \mathcal{H} g+\mathcal{H}(f g) .} \\
\pi_{0} f=\frac{1}{2 \pi} \int_{-\pi}^{\pi} f(x) d x \text { and } \Pi f=f-\pi_{0} f .
\end{gathered}
$$

We then have immediately for any $f, g$ in $L_{\natural}^{2}$

$$
[\mathcal{H} f, \mathcal{H} g]=[\Pi f, \Pi g], \quad \mathcal{H}^{2} f=-\Pi f, \quad \mathcal{H}(\Pi f)=\mathcal{H} f
$$

and

$$
\mathcal{H}(f \mathcal{H} g)+\mathcal{H}(g \mathcal{H} f)-(\mathcal{H} f)(\mathcal{H} g)+f g=\left(\pi_{0} f\right)\left(\pi_{0} g\right)
$$

Now suppose that $w \in L_{\text {如 }}^{2}$ is sufficiently smooth and, for any $f \in L_{\text {如 }}^{2}$, let

$$
\begin{aligned}
L_{w^{\prime}} f & =f+\left[f, w^{\prime}\right], \\
M_{w^{\prime}} f & =f+J\left(f, w^{\prime}\right), \\
\widetilde{L}_{w^{\prime}} f & =f+f \mathcal{H} w^{\prime}+w^{\prime} \mathcal{H} f, \\
\widetilde{M}_{w^{\prime}} f & =f+f \mathcal{H} w^{\prime}-\mathcal{H}\left(w^{\prime} f\right) .
\end{aligned}
$$

Lemma 3.1. Suppose that $w \in L_{\text {如 }}^{2}$ is smooth and $\left|\mathcal{H} w^{\prime}\right|<1$ on $\mathbb{R} \times \mathbb{R}$. Let

$$
D \stackrel{\text { def }}{=}\left(1+\mathcal{H} w^{\prime}\right)^{2}+w^{\prime 2} \neq 0 \text { on }[0,2 \pi] \times[0,2 \pi] .
$$

Then the $t$-dependent bounded linear operators $L_{w^{\prime}}, M_{w^{\prime}}, \widetilde{L}_{w^{\prime}}, \widetilde{M}_{w^{\prime}}$ on $L_{\natural}^{2}$ are smooth functions of $t$. Moreover we have the following.

i) For any fixed $t$,

$$
L_{w^{\prime}}^{*}=M_{w^{\prime}}, \widetilde{L}_{w^{\prime}}^{*}=\widetilde{M}_{w^{\prime}} \quad \text { in } \mathcal{L}\left(L_{\natural}^{2}\right)
$$


and for sufficiently smooth functions $f, g, u$

$$
\begin{aligned}
\langle[f, g], u\rangle & =\langle f, J(u, g)\rangle=-\langle g, J(u, f)\rangle \\
\mathcal{H} \widetilde{L}_{w^{\prime}} f & =\widetilde{L}_{w^{\prime}} \mathcal{H} f-w^{\prime} \pi_{0} f \\
\mathcal{H} \widetilde{M}_{w^{\prime}} f & =\widetilde{M}_{w^{\prime}} \mathcal{H} f-\pi_{0}\left(w^{\prime} f\right) .
\end{aligned}
$$

ii) $L_{w^{\prime}}$ has a bounded inverse in $L_{\sharp}^{2}$ which is given by

$$
L_{w^{\prime}}^{-1} f=\widetilde{L}_{w^{\prime}}\left(\frac{f}{D}\right)
$$

and, for $f \in L_{\natural}^{2}$,

$$
\begin{aligned}
\widetilde{L}_{w^{\prime}}^{-1} f & =\frac{1}{D} L_{w^{\prime}} f, \\
\mathcal{H}\left(\frac{1}{D} L_{w^{\prime}} f\right) & =\frac{1}{D} L_{w^{\prime}} \mathcal{H} f+\frac{w^{\prime}}{D} \pi_{0} f .
\end{aligned}
$$

iii) $M_{w^{\prime}}$ has a bounded inverse on $L_{\natural}^{2}$ and

$$
\begin{aligned}
& M_{w^{\prime}}^{-1} f=\frac{1}{D} \widetilde{M}_{w^{\prime}} f, \quad \widetilde{M}_{w^{\prime}}^{-1} f=M_{w^{\prime}}\left(\frac{f}{D}\right), \\
& M_{w^{\prime}}\left(\frac{1}{D} \mathcal{H} f\right)=\mathcal{H} M_{w^{\prime}}\left(\frac{f}{D}\right)-\pi_{0}\left(\frac{w^{\prime}}{D} f\right) .
\end{aligned}
$$

In addition, for any $(f, g) \in L_{\natural}^{2} \times H_{\natural}^{1}$ or $H_{\natural}^{1} \times L_{\natural}^{2}$

$$
M_{w^{\prime}}^{-1} J(f, g)=\frac{1}{D} \mathcal{H}\left(f L_{w^{\prime}} g\right)+f \mathcal{H}\left(\frac{1}{D} L_{w^{\prime}} g\right)
$$

and, $f$ in $L_{\natural}^{2}$,

$$
\left(L_{w^{\prime}}^{-1}-M_{w^{\prime}}^{-1}\right) f=w^{\prime} \mathcal{H}\left(\frac{f}{D}\right)+\frac{1}{D} \mathcal{H}\left(f w^{\prime}\right)=M_{w^{\prime}}^{-1} J\left(w^{\prime}, L_{w^{\prime}}^{-1} f\right) .
$$

Proof. See Appendix A.

Lemma 3.2. For any $f, g \in H_{\natural}^{k} k \geq 2$,

$$
[g, f]-\left(L_{w^{\prime}} g\right) \mathcal{H}\left(\frac{L_{w^{\prime}} f}{D}\right)+\left(L_{w^{\prime}} f\right) \mathcal{H}\left(\frac{L_{w^{\prime}} g}{D}\right)=0,
$$

in other words,

$$
\frac{[g, f]}{D}=\left[\frac{L_{w^{\prime}} g}{D}, \frac{L_{w^{\prime}} f}{D}\right] .
$$


Proof. From (3.8) we know that

$$
\mathcal{H}\left(\frac{L_{w^{\prime}} f}{D}\right)=\frac{1}{D} L_{w^{\prime}} \mathcal{H} f+\frac{w^{\prime}}{D} \pi_{0} f
$$

and hence

$$
\begin{aligned}
& -\left(L_{w^{\prime}} g\right) \mathcal{H}\left(\frac{L_{w^{\prime}} f}{D}\right)+\left(L_{w^{\prime}} f\right) \mathcal{H}\left(\frac{L_{w^{\prime}} g}{D}\right) \\
& =\frac{L_{w^{\prime}} f}{D}\left\{\left(1+\mathcal{H} w^{\prime}\right) \mathcal{H} g+w^{\prime} g\right\}-\frac{L_{w^{\prime}} g}{D}\left\{\left(1+\mathcal{H} w^{\prime}\right) \mathcal{H} f+w^{\prime} f\right\} \\
& =\frac{1}{D}\left(\left(1+\mathcal{H} w^{\prime}\right)^{2}+w^{\prime 2}\right)[f, g]=-[g, f],
\end{aligned}
$$

and the lemma is proved.

\section{Main Equation}

In this notation the system (K) and (D) can be re-written

$$
\begin{aligned}
\dot{w}+\left[\dot{w}, w^{\prime}\right]-\mathcal{H} \varphi^{\prime} & =0, \\
\dot{\varphi}-\mu w+J\left(\dot{\varphi}-\mu w, w^{\prime}\right)-J\left(\varphi^{\prime}, \dot{w}\right) & =0,
\end{aligned}
$$

and then as

$$
\begin{aligned}
L_{w^{\prime}} \dot{w}-\mathcal{H} \varphi^{\prime} & =0, \\
M_{w^{\prime}}(\dot{\varphi}-\mu w)-J\left(\varphi^{\prime}, \dot{w}\right) & =0 .
\end{aligned}
$$

As a consequence of Lemma 3.1 and $(\mathrm{k}), \varphi$ can be eliminated from (d) to yield a single second order nonlocal PDE for the function $w$ :

$$
\partial_{t}\left(L_{w^{\prime}} \dot{w}\right)-\mu \mathcal{H} w^{\prime}+\mathcal{H} \partial_{x} M_{w^{\prime}}^{-1} J\left(\mathcal{H} L_{w^{\prime}} \dot{w}, \dot{w}\right)=0 .
$$

To see that nothing has been lost in this manoeuvre, suppose $w$ satisfies (4.1). Then

$$
\pi_{0}\left(\partial_{t}\left(L_{w^{\prime}} \dot{w}\right)\right)=0
$$

and, since $\pi_{0}\left(L_{w^{\prime}} \dot{w}\right)$ is odd in $t$ and has zero derivative, we have

$$
\pi_{0}\left(L_{w^{\prime}} \dot{w}\right)=0
$$

and there exists $\varphi(x, t)$ (periodic in $x$, and defined up to an arbitrary function of $t$ ) such that

$$
L_{w^{\prime}} \dot{w}=\mathcal{H} \varphi^{\prime} .
$$

Substituting this into (4.1) yields

$$
\partial_{t}\left(\mathcal{H} \varphi^{\prime}\right)-\mu \mathcal{H} w^{\prime}-\mathcal{H} \partial_{x} M_{w^{\prime}}^{-1} J\left(\varphi^{\prime}, \dot{w}\right)=0,
$$


and hence

$$
\partial_{t} \varphi-\mu w-M_{w^{\prime}}^{-1} J\left(\varphi^{\prime}, \dot{w}\right)=: c(t) .
$$

Define $d(t)$ such that $\dot{d}=-c$, then

$$
\partial_{t}(\varphi+d)-\mu w-M_{w^{\prime}}^{-1} J\left((\varphi+d)^{\prime}, \dot{w}\right)=0,
$$

which shows that $\partial_{t}(\varphi+d)$ is periodic in $t$ and $x$. Finally

$$
\begin{aligned}
M_{w^{\prime}}\left(\partial_{t}(\varphi+d)-\mu w\right)-J\left((\varphi+d)^{\prime}, \dot{w}\right) & =0, \\
L_{w^{\prime}} \dot{w}-\mathcal{H}(\varphi+d)^{\prime} & =0,
\end{aligned}
$$

which shows that $(\mathrm{k})$ and $(\mathrm{d})$ are satisfied by $(w, \varphi+d)$. Hence nothing is lost in considering equation (4.1).

Remark 4.1. Care must be exercised because any constant can be added to a solution of (4.1) to obtain another solution. In particular, any constant is a solution. To find non-constant solutions we work in a subspace, to be determined later, and in a neighbourhood of the non-constant approximate solution introduced in Section 4.2.

\subsection{Linearization at the origin}

When (4.1) is written as

$$
\mathcal{F}(w, \mu)=0 \text { where } \mathcal{F}(w, \mu)=\mathcal{L}_{\mu}(w)+\mathcal{N}(w),
$$

the linearization of $\mathcal{F}(\cdot, \mu)$ at the origin is given by

$$
\mathcal{L}_{\mu} u \stackrel{\text { def }}{=} D_{u} \mathcal{F}(0, \mu) u=\ddot{u}-\mu \mathcal{H} u^{\prime} .
$$

For $j \geq 2$ let $\mathcal{N}_{j}$ be given by

$$
\mathcal{N}_{j}(u, \cdots, u)=\frac{1}{j !} \frac{\partial^{j} \mathcal{F}(\cdot, \mu)}{\partial u^{j}}(0)(u, \cdots, u)=\frac{1}{j !} \frac{\partial^{j} \mathcal{N}}{\partial u^{j}}(0)(u, \cdots, u) .
$$

Then the quadratic and cubic terms in the Taylor expansion of $\mathcal{F}(\cdot, \mu)$ at 0 follow easily from (3.10) (see Appendix (B.1) for the terms of order four):

$$
\begin{aligned}
\mathcal{N}_{2}(u, u)= & \partial_{t}\left[\dot{u}, u^{\prime}\right]+\frac{1}{2} \mathcal{H} \partial_{x}\left\{3(\mathcal{H} \dot{u})^{2}-\dot{u}^{2}\right\} \\
= & \partial_{t}\left[\dot{u}, u^{\prime}\right]+\partial_{x}\left(\mathcal{H}\left(\dot{u}^{2}\right)-3 \dot{u} \mathcal{H} \dot{u}\right), \\
\mathcal{N}_{3}(u, u, u)= & \mathcal{H} \partial_{x}\left\{-\left(\mathcal{H} u^{\prime}\right)\left((\mathcal{H} \dot{u})^{2}-\dot{u}^{2}+\left(\pi_{0} \dot{u}\right)^{2}\right)-2(\mathcal{H} \dot{u}) \mathcal{H}\left(\dot{u} \mathcal{H} u^{\prime}\right)\right. \\
& \left.+3(\mathcal{H} \dot{u}) \mathcal{H}\left[\dot{u}, u^{\prime}\right]-\dot{u}\left[\dot{u}, u^{\prime}\right]\right\} \\
= & -\mathcal{H} \partial_{x}\left\{4(\mathcal{H} \dot{u})\left(\mathcal{H}\left(u^{\prime} \mathcal{H} \dot{u}\right)\right\}+u^{\prime \prime}\left(\pi_{0} \dot{u}\right)^{2},\right.
\end{aligned}
$$


The following are suitable spaces in which to study our nonlinear system. For any $s \in \mathbb{N}_{0}$ we denote by $H_{\text {比 }}^{s}$ the Hilbert space of $2 \pi$-periodic functions $u$ : $\mathbb{R}^{2} \rightarrow \mathbb{R}$ with norm

$$
\|u\|_{s}=\left\{\sum_{(m, n) \in \mathbb{Z}^{2}}(1+|(m, n)|)^{2 s}|\mathfrak{F} u(m, n)|^{2}\right\}^{1 / 2}
$$

where the Fourier transform of $u$ is

$$
\mathfrak{F} u(m, n)=\frac{1}{4 \pi^{2}} \int_{-\pi}^{\pi} \int_{-\pi}^{\pi} \exp (-i(m, n) \cdot(x, t)) u(x, t) d x d t, \quad(m, n) \in \mathbb{Z}^{2} .
$$

For $m \geq 0$ let

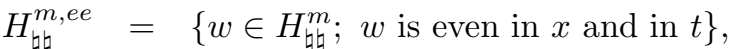

$$
\begin{aligned}
& H_{\text {如 }}^{m, e o}=\left\{\varphi \in H_{\text {弘 }}^{m} ; \varphi \text { is even in } x \text { and odd in } t\right\} \text {, }
\end{aligned}
$$

and so on, depending on evenness or oddness with respect to $x$ and to $t$. Then, for $m \geq 4, \mathcal{F}$ is an analytic map from $H_{\text {如 }}^{m, e e} \times \mathbb{R}$ to $H_{\text {响 }}^{m-2, e e}$, the linear operator $\mathcal{L}_{\mu}$ is bounded from $H_{\text {响 }}^{m, e e}$ to $H_{\text {响 }}^{m-2, e e}$, for $m \geq 2$, and the nonlinear operator $\mathcal{N}$ is analytic from $H_{\text {如 }}^{m, e e}$ to $H_{\text {吅 }}^{m-2 \text {,ee }}$ for $m \geq 3$. This last property comes from the fact that $H_{\text {如 }}^{m}$ is an algebra for $m \geq 2$ (we are working in two dimensions). However, this choice of spaces is not optimal for the linear operator, since $w^{\prime \prime}$ appears in the nonlinear, but not in the linear term. Hence we cannot expect that an inverse of $\mathcal{L}_{\mu}$, applied to the nonlinear term, regains this loss of differentiability. In fact the situation is even worse because of a small divisor problem; see Remark 4.4. Consider (4.2) linearized at the origin:

$$
\mathcal{L}_{\mu} u=f \text { for } f \in H_{\text {如 }}^{m, e e}, m \geq 0 .
$$

Let $u_{n}, n \geq 0$, denote the Fourier cosine coefficients with respect to $x$ of $u$. Then (4.4) implies that

$$
\ddot{u}_{n}+\mu n u_{n}=f_{n},
$$

where

$$
\begin{aligned}
& u_{n}(t)=\frac{1}{\pi} \int_{-\pi}^{\pi} u(x, t) \cos n x d x, n \geq 1 \\
& u_{0}(t)=\left(\pi_{0} u\right)(t)=\frac{1}{2 \pi} \int_{-\pi}^{\pi} u(x, t) d x
\end{aligned}
$$

Let

$$
u_{n}^{(q)}=\frac{1}{\pi} \int_{-\pi}^{\pi} u_{n}(t) \cos q t d t, q \geq 1,
$$

the Fourier cosine coefficients of $u_{n}$ with respect to $t$. Then, after taking the Fourier coefficients in $t$, we have

$$
\left(n \mu-q^{2}\right) u_{n}^{(q)}=f_{n}^{(q)},
$$

This leads to the following observation on the spectrum of the linearized problem. 
Lemma 4.2. In $H_{\natural \text { ma }}^{m, e e}$, the kernel of $\mathcal{L}_{\mu}$ consists of constants when $\mu \notin \mathbb{Q}$. On the other hand, in $H_{\natural \natural}^{m, e e}$ the kernel of $\mathcal{L}_{1}$ is $E_{0} \cap H_{\natural \natural}^{m, e e}$, where

$$
E_{0}=\operatorname{span}\left\{A_{q} \cos q^{2} x \cos q t: A_{q} \in \mathbb{R}, \quad q \in \mathbb{N} \cup\{0\}\right\} .
$$

For all other $\mu \in \mathbb{Q}$, the kernel of $\mathcal{L}_{\mu}$ is infinite dimensional (and is easily deduced from $E_{0}$ ).

Proof. Note that any element of $\mathbb{Q}$ can be written as $q^{2} / n$. From (4.7) we find that if $\mu \neq q^{2} / n$, the kernel is trivial For $\mu=1$, the kernel is obtained by considering all $n, q$ with $n=q^{2}$. (For rational values of $\mu$ of the form $q^{2} / n$, the calculation of the kernel is left to the reader.)

Now we focus on values of $\mu$ where the kernel is non-trivial. In fact we concentrate on the case $\mu=1$, which is not a restriction since for general $\mu=q^{2} / n$ it suffices to rescale space and time to modify $\mu$ and replace $q^{2} / n$ by 1. Note that $n=0$ and $\mu=1$ in (4.5), leads to the compatibility condition

$$
\int_{0}^{2 \pi} f_{0}(t) d t=0
$$

and $u_{0}(t)$ with $\int_{0}^{2 \pi} u_{0}(t) d t=0$ is then given by

$$
u_{0}(t)=\int_{0}^{t} \int_{0}^{s} f_{0}(\tau) d \tau d s-\frac{1}{2 \pi} \int_{0}^{2 \pi}\left(\int_{0}^{t} \int_{0}^{s} f_{0}(\tau) d \tau d s\right) d t .
$$

For other values of $n \neq q^{2}, q \in \mathbb{N} \cup\{0\}$ and $\mu=1$,

$$
\begin{aligned}
u_{n}(t)= & \frac{1}{2 \sqrt{n} \sin \pi \sqrt{n}} \int_{0}^{2 \pi} f_{n}(s) \cos \sqrt{n}(\pi+t-s) d s+ \\
& +\frac{1}{\sqrt{n}} \int_{0}^{t} f_{n}(s) \sin \sqrt{n}(t-s) d s .
\end{aligned}
$$

For $n=q^{2} \neq 0$, the compatibility condition $f_{q^{2}}^{(q)}=0$ must be satisfied and we have

$$
u_{q^{2}}(t)=\frac{1}{q} \int_{0}^{t} f_{q^{2}}(s) \sin q(t-s) d s .
$$

In $L_{\text {घ中 }}^{2}$ the orthogonal projection $\widetilde{P}_{0}$ onto the kernel of $\mathcal{L}_{1}$ is defined by

$$
\widetilde{P}_{0} u=\sum_{q \in \mathbb{N} \cup\{0\}} u_{q^{2}}^{(q)} \cos q^{2} x \cos q t \text { for } u(x, t)=\sum_{n, q \in \mathbb{N} \cup\{0\}} u_{n}^{(q)} \cos n x \cos q t .
$$

Lemma 4.3. For any $f \in H_{\text {如 }}^{m \text { ee }}$ with $\widetilde{P}_{0} f=0$ there is a unique $u$ which satisfies

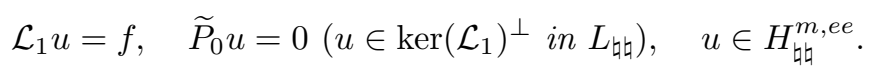


Proof. We use the fact [13, Remark 4.2] that for any $n \in \mathbb{N} \cup\{0\}$ with $n \neq q^{2}$ for all $q$,

$$
\sqrt{n}|\sin \pi \sqrt{n}|>\pi / 2-\varepsilon_{n}, \quad 0<\varepsilon_{n}=O\left(n^{-1 / 2}\right) \text { as } n \rightarrow \infty .
$$

It then follows from the above formulae that, for $n \neq q^{2}$,

$$
\begin{aligned}
\left\|u_{n}\right\|_{L^{2}} & \leq C|| f_{n} \|_{L^{2}}, \\
\left\|\dot{u}_{n}\left(\cdot+\frac{\pi}{2 \sqrt{n}}\right)+\sqrt{n} u_{n}\right\|_{L^{2}} & \leq C|| f_{n} \|_{L^{2}},
\end{aligned}
$$

and, for $n=q^{2}$,

$$
\begin{aligned}
|q|\left\|u_{q^{2}}\right\|_{L^{2}}+\left\|u_{q^{2}}\right\|_{1} & \leq C\left\|f_{q^{2}}\right\|_{L^{2}}, \quad q \neq 0, \\
\left\|u_{0}\right\|_{2} & \leq C\left\|f_{0}\right\|_{L^{2}}, \quad q=0,
\end{aligned}
$$

and the result of the lemma follows.

Remark 4.4. Observe that in this lemma we do not gain enough regularity to compensate for the loss of regularity due to the nonlinear terms (second order derivatives in $x$ and in $x t$ ). As a consequence, we resort to the Nash-Moser implicit function theorem in dealing with the bifurcation problem.

\subsection{Approximate solutions}

Solutions of the standing-wave problem as a formal power series in $\epsilon$, where $\mu=1+\varepsilon^{2} / 4$, are known to exist and all the coefficients can be calculated in closed form by an explicit algorithm [1, 14]. Although the convergence of the resulting power series is unknown, this observation leads to approximate solutions of the standing-wave problem, formally accurate up to arbitrary power of $\varepsilon>0$ sufficiently small. Here we recall the details of this calculation in the context of equation (4.1).

Lemma 4.5. An approximate solution $w$ of (4.1) is given by

$$
w_{\varepsilon}^{(N)}=\sum_{1 \leq n \leq N} \varepsilon^{n} w^{(n)}, \quad \mu-1=\varepsilon^{2} / 4,
$$

where all the $w^{(n)}$ can be calculated explicitly,

$$
\begin{aligned}
& w^{(1)}=\cos x \cos t \\
& w^{(2)}=\frac{1}{4} \cos 2 t-\frac{1}{2} \cos 2 x(1+\cos 2 t) \\
& w^{(3)}=-\frac{37}{32} \cos x \cos t-\frac{11}{32} \cos x \cos 3 t+\frac{3}{8} \cos 3 x(3 \cos t+\cos 3 t)
\end{aligned}
$$

and, if equation (4.1) is re-written as (4.2), then

$$
\mathcal{F}\left(w_{\varepsilon}^{(N)}, 1+\varepsilon^{2} / 4\right)=: \varepsilon^{N+1} Q_{\varepsilon},
$$

where $Q_{\varepsilon}$ is bounded in $H_{\text {吅 }}^{m, e e}$, for all $m$, as $\varepsilon \rightarrow 0$. 
Proof. Equation (4.1) may be written as

$$
\mathcal{L}_{1} w-\nu \mathcal{H} w^{\prime}+\mathcal{N}_{2}(w, w)+\mathcal{N}_{3}(w, w, w)+\ldots=0,
$$

where $\mu=1+\nu$. We know from [1] that there exists, for any $N \in \mathbb{N}$, an approximate solution of the form

$$
w_{\varepsilon}^{(N)}=\sum_{0 \leq n \leq N} \varepsilon^{n} w^{(n)}, \quad \nu=\varepsilon^{2} / 4 \text { with } w^{(1)}=\cos x \cos t .
$$

The results is that there exists a formal expansion of a solution of (4.8) in the form

$$
w=\sum_{n \geq 1} \varepsilon^{n} w^{(n)}, \quad \mu=1+\varepsilon^{2} / 4,
$$

where

$$
\begin{aligned}
w^{(2 m)} & =\sum_{0 \leq p, q \leq m} w_{p, q}^{(2 m)} \cos 2 q t \cos 2 p x, \quad w_{0,0}^{(2 m)}=0, \\
w^{(2 m+1)} & =\sum_{0 \leq p, q \leq m} w_{p, q}^{(2 m+1)} \cos (2 q+1) t \cos (2 p+1) x,
\end{aligned}
$$

which gives the structure of approximate solutions up to any fixed order $\varepsilon^{N}$. The calculation of $w^{(2)}, w^{(3)}$, and a sketch calculation for $w^{(4)}$ and $w^{(5)}$, are given in Appendix B.

Remark. The existence of an exact formal power series for a standing wave was originally given for an equivalent formulation of the problem in [1], and for a more general type of standing waves in [14]. It is worth noting that, for the proofs in this paper, we need only the coefficients of the approximate solution $w$ of $\mathcal{F}(w, \mu)=0$ with $N=2$ and that the coefficient of $\cos x \cos t$ in $w_{\varepsilon}^{(3)}$ only influences $\mathcal{F}$ at orders $\varepsilon^{4}$ and above. Computations made in Appendix B are sufficient for our purpose.

\subsection{An equation for standing waves}

Fixed $N_{0} \in \mathbb{N}$. Then define $\Phi$ on $H_{\text {如 }}^{m, e e} \times \mathbb{R}$ by

$$
\Phi(u, \varepsilon)=\frac{1}{\varepsilon^{N_{0}}} \mathcal{F}\left(w_{\varepsilon}^{\left(N_{0}\right)}+\varepsilon^{N_{0}} u, 1+\frac{\varepsilon^{2}}{4}\right), \quad \varepsilon \neq 0, \quad \Phi(u, 0)=0
$$

and seek solutions of

$$
\Phi(u, \varepsilon)=0, \quad \int_{0}^{2 \pi} \int_{0}^{2 \pi} u(x, t) d x d t=0 .
$$

To do so we use the Nash-Moser theorem in Appendix N restricted to functions with zero mean in $H_{\text {好 }}^{r, e e}$, for some $r$. Note that $w_{\varepsilon}^{(n)}$ has zero mean and, for $N \in \mathbb{N}$,

$$
w_{\varepsilon}^{\left(N_{0}+N\right)}=w_{\varepsilon}^{\left(N_{0}\right)}+\varepsilon^{N_{0}}\left(\varepsilon \sum_{n=N_{0}+1}^{N_{0}+N} \varepsilon^{n-N_{0}-1} w^{(n)}\right)=: w_{\varepsilon}^{\left(N_{0}\right)}+\varepsilon^{N_{0}}\left(u_{\varepsilon}^{(N)}\right),
$$


(this is the definition of $u_{\varepsilon}^{(N)}$ ),

$$
\left\|u_{\varepsilon}^{(N)}\right\|_{m} \leq \text { const }|\varepsilon| \text { and }\left\|\Phi\left(u_{\varepsilon}^{(N)}, \varepsilon\right)\right\|_{m} \leq \text { const } \varepsilon^{N+1},
$$

where the constants depend on $m$ and $N$. This shows that $\Phi$ satisfies hypothesis (N.3) and (N.4) and (see Remark 4.1) solutions $u \neq 0, \varepsilon \neq 0$ sufficiently small, give solutions of the standing-wave problem. It is straightforward to see that when $\mu$ and $\|w\|_{3}$ are bounded, by $M_{3}$ say, then, for all $l \in \mathbb{N}_{0}$ (where $\mathbb{N}_{0}$ denotes the set of integers $\geq 0)$, there is a constant $c_{l}\left(M_{3}\right)$ such that

$$
\|\mathcal{F}(w, \mu)\|_{l+1} \leq c_{l}\left(M_{3}\right)\|w\|_{l+3} .
$$

This is enough to ensure that $\Phi$ satisfies (N.1a) with $\rho=r-3 \geq 1$. In the notation of Appendix $\mathrm{N}$ the derivative of $\Phi$ in the direction $\underline{u}$ at an arbitrary point $(u, \varepsilon)$ is given by

$$
\Phi_{u}^{\prime}(u, \varepsilon) \underline{u}=\partial_{w} \mathcal{F}\left(w_{\varepsilon}^{\left(N_{0}\right)}+\varepsilon^{N_{0}} u, 1+\frac{\varepsilon^{2}}{4}\right) \underline{u}
$$

and to apply the Nash-Moser implicit function theorem to (4.10) we need to invert an operator $\Lambda(u, \varepsilon)$ that approximates the operator (4.12) at an arbitrary point $(u, \varepsilon)$ in the sense of (N.1d) and (N.1e). In particular $\Lambda(u, \varepsilon)$ and $\Phi_{u}^{\prime}(u, \varepsilon)$ coincide when $(u, \varepsilon)$ satisfies (4.10). For more detail of how this is achieved, see Sections 6.1 and 8.1.

\section{$5 \quad$ Linearization at a Non-zero Point}

The analysis of this section leads to an operator $\Lambda$, given explicitly by (6.3) below, which coincides with $\Phi_{u}^{\prime}$ at solutions of (4.10) and which we eventually show has all the properties required in Appendix N. To find estimates on the inverse of $\Lambda(u, \varepsilon)$ at arbitrary points we will see it is equivalent to estimate the inverse of $\mathcal{L}\left(w, 1+\varepsilon^{2} / 4\right)$, given by (6.2), at any point $w \neq 0$. So consider the linear problem for a function $\delta w$,

$$
\partial_{w} \mathcal{F}(w, \mu) \delta w=f
$$

where $\mathcal{F}$ is defined when (4.1) is written as (4.2). Note that $\mathcal{F}$ maps $C_{\text {如 }}^{m, e e}$ to

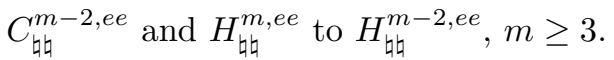

Lemma 5.1. In (5.1) suppose that $w \in H_{\text {如 }}^{\text {,ee }} \backslash\{0\}, m \geq 4$, and $\left|\mathcal{H} w^{\prime}\right|<1$. Let $v=L_{w^{\prime}}(\delta w)$. Then

$$
\ddot{v}-\mu \mathcal{H} v^{\prime}+\mathcal{H} \partial_{x}\left(\mathcal{Q} \dot{v}+\mathcal{R} v^{\prime}+\mathcal{T}_{\mu} v\right)=f .
$$

Here $\mathcal{Q}, \mathcal{R}, \mathcal{T}_{\mu}$ are the bounded linear operators from $H_{\text {婚 }}^{k}$ to itself, $k \leq m-2$, 
which depend smoothly on $t$, given by

$$
\begin{aligned}
\mathcal{Q} g= & J(a, g)+\left(\frac{\pi_{0}(g)}{D}-\pi_{0}\left(\frac{g}{D}\right)\right) \pi_{0}\left(L_{w^{\prime}} \dot{w}\right) \\
= & a \mathcal{H} g+\mathcal{H}(a g)+\left(\frac{\pi_{0}(g)}{D}-\pi_{0}\left(\frac{g}{D}\right)\right) \int_{0}^{t} \pi_{0}(\mathcal{F}(w, \mu))(s) d s, \\
\mathcal{R} g= & -a \mathcal{H}(a g)+\left(a \pi_{0}\left(\frac{g}{D}\right)-\frac{\pi_{0}(a g)}{D}\right)\left(\int_{0}^{t} \pi_{0}(\mathcal{F}(w, \mu))(s) d s\right) \\
& -\frac{1}{D} \mathcal{H}\left(\frac{g}{D}\right)\left(\int_{0}^{t} \pi_{0}(\mathcal{F}(w, \mu))(s) d s\right)^{2}, \\
\partial_{x} \mathcal{H} \mathcal{T}_{\mu} g= & \partial_{x} \mathcal{H}\left\{-a \mathcal{H}\left(a^{\prime} g\right)+\mathcal{H}(\dot{a} g)-\mathcal{H} \mathcal{F}(w, \mu) \mathcal{H}(g / D)+b g\right. \\
& -\frac{1}{D} \pi_{0}\left(a^{\prime} g\right) \int_{0}^{t} \pi_{0}(\mathcal{F}(w, \mu))(s) d s \\
- & \left.\frac{1}{D} \mathcal{H}\left(\left(\frac{1}{D}\right)^{\prime} g\right)\left(\int_{0}^{t} \pi_{0}(\mathcal{F}(w, \mu))(s) d s\right)^{2}\right\}
\end{aligned}
$$

where $\mathcal{H}(\mathcal{F}(w, \mu)) \in H_{\text {吅 }}^{m-2, o e}$,

$$
\begin{aligned}
a= & J\left(\frac{1}{D}, L_{w^{\prime}} \dot{w}\right)=2 M_{w^{\prime}}^{-1} \mathcal{H} \dot{w}=\mathcal{H}\left(\frac{1}{D} L_{w^{\prime}} \dot{w}\right)+\frac{1}{D} \mathcal{H}\left(L_{w^{\prime}} \dot{w}\right) \in H_{\text {如 }}^{m-1, o o}, \\
b= & D^{-1}\left\{a^{2} L_{w^{\prime}} w^{\prime \prime}-2 a L_{w^{\prime}} \dot{w}^{\prime}+L_{w^{\prime}} \ddot{w}+\mu\left(D-1-\mathcal{H} w^{\prime}\right)\right\} \\
& +\frac{L_{w^{\prime}} w^{\prime \prime}}{D^{3}}\left(\pi_{0} L_{w^{\prime}} \dot{w}\right)^{2} \in H_{\text {如 }}^{m-2, e e},
\end{aligned}
$$

and

$$
\mathcal{Q}^{*}=-\mathcal{Q}, \mathcal{R}^{*}=-\mathcal{R}
$$

If $|\mu|+\|w\|_{3} \leq M_{3}$, there exist constants $c_{l}\left(M_{3}\right), l \in \mathbb{N}_{0}$, such that

$$
\|a\|_{l+2} \leq c_{l}\left(M_{3}\right)\|w\|_{l+3}, \quad\|b\|_{l+2} \leq c_{l}\left(M_{3}\right)\|w\|_{l+4} .
$$

Moreover, if $w$ is an approximate solution of (4.1) (or equivalently (4.2)), at order $\varepsilon^{2}$, and $\mu=1+\varepsilon^{2} / 4$, then for any integer $m,\|\mathcal{F}(w, \mu)\|_{m}=O\left(\varepsilon^{3}\right)$ as $\varepsilon \rightarrow 0$ and

$$
\begin{aligned}
a & =2 \varepsilon \sin x \sin t-\varepsilon^{2} \sin 2 x \sin 2 t+O\left(\varepsilon^{3}\right), \\
b & =-2 \varepsilon \cos x \cos t+\varepsilon^{2}(-1+\cos 2 x(1+\cos 2 t))+O\left(\varepsilon^{3}\right) .
\end{aligned}
$$

Remark. Note that the linear operator $\mathcal{Q} \dot{v}+\mathcal{R} v^{\prime}-a \mathcal{H}\left(a^{\prime} v\right)+\mathcal{H}(\dot{a} v)+b v$ is

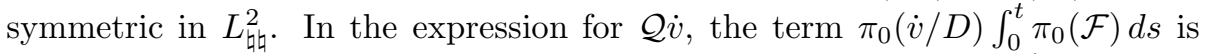
independent of $x$ and so is in the kernel of $\mathcal{H} \partial_{x}$. Similarly in $\mathcal{R} v^{\prime}$, the term 
$a \pi_{0}\left(v^{\prime} / D\right) \int_{0}^{t} \pi_{0}(\mathcal{F}) d s$ is zero since $v^{\prime} / D$ is odd in $x$. Thus

$$
\begin{aligned}
\partial_{x} \mathcal{H}\left\{\mathcal{Q} \dot{v}+\mathcal{R} v^{\prime}\right. & \left.+\mathcal{T}_{\mu} v\right\}=\partial_{x} \mathcal{H}\left\{a \mathcal{H}\left\{\dot{v}-\partial_{x}(a v)\right\}+\mathcal{H} \partial_{t}(a v)+b v\right. \\
& +\frac{1}{D}\left(\pi_{0} \dot{v}\right) \int_{0}^{t} \pi_{0}(\mathcal{F}(w, \mu)) d s-\mathcal{H}(\mathcal{F}(w, \mu)) \mathcal{H}(v / D) \\
& \left.-\frac{1}{D} \mathcal{H} \partial_{x}(v / D)\left\{\int_{0}^{t} \pi_{0}(\mathcal{F}(w, \mu)) d s\right\}^{2}\right\}
\end{aligned}
$$

Therefore when $w$ is a solution of (4.1), the linearised problem is simply

$$
\ddot{v}-\mu \mathcal{H} v^{\prime}+\partial_{x} \mathcal{H}\left\{a \mathcal{H}\left\{\dot{v}-\partial_{x}(a v)\right\}+\mathcal{H} \partial_{t}(a v)+b v\right\}=f .
$$

Proof. To simplify notation we introduce an intermediate variable $\phi$ defined by

$$
\phi^{\prime}=-\mathcal{H} L_{w^{\prime}} \dot{w}, \quad \phi(0, t)=0,
$$

and, denote by $\psi$ the increment in $\phi$ due to an increment $\delta w$ in $w$. Then

$$
\psi^{\prime}=-\mathcal{H}\left(L_{w^{\prime}} \dot{\delta} w+\left[\dot{w}, \delta w^{\prime}\right]\right) .
$$

In (5.7) we make a change of variables

$$
v=L_{w^{\prime}}(\delta w), \quad \theta=\psi+\mathcal{H}[\dot{w}, \delta w]
$$

which yields

$$
\mathcal{H} \dot{v}+\theta^{\prime}=0, \quad \dot{\psi}=\dot{\theta}+\mathcal{H} \partial_{t}\left[L_{w^{\prime}}^{-1} v, \dot{w}\right] \text { and } \psi^{\prime}=-\mathcal{H} \dot{v}+\mathcal{H} \partial_{x}\left[L_{w^{\prime}}^{-1} v, \dot{w}\right] .
$$

Note, from the first identity, that

$$
\dot{v}=\mathcal{H} \theta^{\prime}+\partial_{t} \pi_{0}\left(\delta w\left(1+2 \mathcal{H} w^{\prime}\right)\right)
$$

and that $L_{w^{\prime}} \dot{w}=\pi_{0}\left(L_{w^{\prime}} \dot{w}\right)+\mathcal{H} \phi^{\prime}$ where

$$
\pi_{0}\left(L_{w^{\prime}} \dot{w}\right)=\frac{1}{2 \pi} \int_{0}^{2 \pi}\left(\dot{w}+\dot{w} \mathcal{H} w^{\prime}-w^{\prime} \mathcal{H} \dot{w}\right) d x=\frac{d}{d t}\left(\frac{1}{2 \pi} \int_{0}^{2 \pi} w\left(1+\mathcal{H} w^{\prime}\right) d x\right) .
$$

In this notation (4.1) can be written as

$$
\mathcal{H} \partial_{x} \mathcal{G}(w, \mu)+\partial_{t t} \pi_{0}\left(w\left(1+\mathcal{H} w^{\prime}\right)\right)=0
$$

where the operator $\mathcal{G}$ is given by

$$
\mathcal{G}(w, \mu)=\dot{\phi}-\mu w-M_{w^{\prime}}^{-1} J\left(\phi^{\prime}, \dot{w}\right) .
$$

The linearization of $\partial_{t t} \pi_{0}\left(w\left(1+\mathcal{H} w^{\prime}\right)\right)$ at $w$ in the direction $\delta w$ is

$$
\partial_{t t} \pi_{0}\left(\delta w\left(1+2 \mathcal{H} w^{\prime}\right)\right) .
$$


The linearization, $\partial_{w} \mathcal{G}(w, \mu) \delta w$ of $\mathcal{G}$ in the direction $\delta w$, is given by

$$
\dot{\psi}-\mu \delta w-M_{w^{\prime}}^{-1} J\left(\psi^{\prime}, \dot{w}\right)-M_{w^{\prime}}^{-1} J\left(\phi^{\prime}, \dot{\delta} \dot{w}\right)+M_{w^{\prime}}^{-1} J\left(M_{w^{\prime}}^{-1} J\left(\phi^{\prime}, \dot{w}\right), \delta w^{\prime}\right),
$$

where $\dot{\delta w}$ and $\delta w^{\prime}$ denote derivatives of $\delta w$ and we have used that

$$
\begin{aligned}
\left(\partial_{w} M_{w^{\prime}}[\delta w]\right) g & =J\left(g, \delta w^{\prime}\right), \\
\left(\partial_{w}\left(M_{w^{\prime}}^{-1}\right)[\delta w]\right) g & =-M_{w^{\prime}}^{-1} J\left(M_{w^{\prime}}^{-1} g, \delta w^{\prime}\right) .
\end{aligned}
$$

Thus in terms of the new variables $(v, \theta)$,

$$
\begin{aligned}
\partial_{w} \mathcal{G}(w, \mu) \delta w=\dot{\theta} & -\mu L_{w^{\prime}}^{-1} v+\partial_{t} \mathcal{H}\left[L_{w^{\prime}}^{-1} v, \dot{w}\right]-M_{w^{\prime}}^{-1} J\left(\phi^{\prime}, \partial_{t}\left(L_{w^{\prime}}^{-1} v\right)\right) \\
+ & M_{w^{\prime}}^{-1} J(\mathcal{H} \dot{v}, \dot{w})+M_{w^{\prime}}^{-1} J\left(M_{w^{\prime}}^{-1} J\left(\phi^{\prime}, \dot{w}\right), \partial_{x}\left(L_{w^{\prime}}^{-1} v\right)\right) \\
& -M_{w^{\prime}}^{-1} J\left(\mathcal{H} \partial_{x}\left[L_{w^{\prime}}^{-1} v, \dot{w}\right], \dot{w}\right) .
\end{aligned}
$$

Now we identify the operators in the linearization that act on $\dot{v}, v^{\prime}$ and $v$. Define bounded operators $\mathcal{Q}, \mathcal{R}, \mathcal{T}_{\mu}$ acting on a function $g$ by

$$
\begin{aligned}
\mathcal{Q} g & =\mathcal{H}\left[L_{w^{\prime}}^{-1} g, \dot{w}\right]+M_{w^{\prime}}^{-1} J(\mathcal{H} g, \dot{w})-M_{w^{\prime}}^{-1} J\left(\phi^{\prime}, L_{w^{\prime}}^{-1} g\right), \\
\mathcal{R} g & =M_{w^{\prime}}^{-1} J\left(M_{w^{\prime}}^{-1} J\left(\phi^{\prime}, \dot{w}\right), L_{w^{\prime}}^{-1} g\right)-M_{w^{\prime}}^{-1} J\left(\mathcal{H}\left[L_{w^{\prime}}^{-1} g, \dot{w}\right], \dot{w}\right), \\
\mathcal{T}_{\mu} g & =\mathcal{T}_{0} g+\mu \mathcal{T}^{(1)} g \\
\mathcal{T}_{0} g & =-\mathcal{Q}\left[L_{w^{\prime}}^{-1} g, \dot{w}^{\prime}\right]-\mathcal{R}\left[L_{w^{\prime}}^{-1} g, w^{\prime \prime}\right]+\mathcal{H}\left[L_{w^{\prime}}^{-1} g, \ddot{w}\right] \\
\mathcal{T}^{(1)} g & =\left(g-L_{w^{\prime}}^{-1} g\right) .
\end{aligned}
$$

Then (5.11) becomes

$$
\partial_{w} \mathcal{G}(w, \mu) \delta w=\dot{\theta}-\mu v+\mathcal{Q} \dot{v}+\mathcal{R} v^{\prime}+\mathcal{T}_{\mu} v,
$$

where we have used that

$$
L_{w^{\prime}}\left(\partial_{t} L_{w^{\prime}}^{-1}\right) g=-\left[L_{w^{\prime}}^{-1} g, \dot{w}^{\prime}\right] \text { and } L_{w^{\prime}}\left(\partial_{x} L_{w^{\prime}}^{-1}\right) g=-\left[L_{w^{\prime}}^{-1} g, w^{\prime \prime}\right] .
$$

In the light of (5.8), (5.9), (5.10) and (5.15), the linearization of (4.1) leads to a linear non-local second order PDE (5.2) for $v$ :

$$
\ddot{v}-\mu \mathcal{H} v^{\prime}+\mathcal{H} \partial_{x}\left(\mathcal{Q} \dot{v}+\mathcal{R} v^{\prime}+\mathcal{T}_{\mu} v\right)=f .
$$

Observe from the symmetry property in Lemma 3.1 (i) that

$$
\mathcal{Q}^{*}=-\mathcal{Q} \text { and } \mathcal{R}^{*}=-\mathcal{R} \text {. }
$$

The following two propositions complete the description of $\mathcal{Q}$ and $\mathcal{R}$.

Proposition 5.2. For any $w$ in $H_{\text {如 }}^{m, e e}$ and $g \in L_{\text {㭃, }}^{2}$,

$$
\begin{aligned}
\mathcal{Q} g & =J(a, g)+\left(\frac{\pi_{0}(g)}{D}-\pi_{0}\left(\frac{g}{D}\right)\right) \pi_{0}\left(L_{w^{\prime}} \dot{w}\right) \\
& =a \mathcal{H} g+\mathcal{H}(a g)+\left(\frac{\pi_{0}(g)}{D}-\pi_{0}\left(\frac{g}{D}\right)\right) \int_{0}^{t} \pi_{0}(\mathcal{F}(w, \mu))(\tau) d \tau
\end{aligned}
$$


where

$$
\begin{aligned}
a & =J\left(\frac{1}{D}, L_{w^{\prime}} \dot{w}\right)=2 M_{w^{\prime}}^{-1} \mathcal{H} \dot{w} \in H_{\text {如 }}^{m-1, o o}, \\
& =\mathcal{H}\left(\frac{1}{D} L_{w^{\prime}} \dot{w}\right)+\frac{1}{D} \mathcal{H}\left(L_{w^{\prime}} \dot{w}\right) \text { is odd in } x \text { and } t .
\end{aligned}
$$

Proof. The proof of this proposition is given in Appendix C.

Proposition 5.3. For any $w$ in $H_{\natural \natural}^{m, e e}$ and $g \in L_{\text {吅, }}^{2}$,

$$
\begin{aligned}
\mathcal{R} g= & -a \mathcal{H}(a g)+\left(a \pi_{0}\left(\frac{g}{D}\right)-\frac{\pi_{0}(a g)}{D}\right)\left(\int_{0}^{t} \pi_{0}(\mathcal{F}(w, \mu))(\tau) d \tau\right) \\
& -\frac{1}{D} \mathcal{H}\left(\frac{g}{D}\right)\left(\int_{0}^{t} \pi_{0}(\mathcal{F}(w, \mu))(\tau) d \tau\right)^{2},
\end{aligned}
$$

where $a \in H_{\mathrm{\natural}}^{m-1, o o}$ is defined in the previous proposition.

Proof. The proof is in Appendix D.

Proof of Lemma 5.1 concluded. Next we give an explicit formula for $\partial_{x} \mathcal{H} \mathcal{T}_{\mu}(v)$. We have, from (5.14) and the above expressions for $\mathcal{Q}$ and $\mathcal{R}$,

$$
\begin{aligned}
\partial_{x} \mathcal{H} \mathcal{T}_{\mu}(v) & =\partial_{x} \mathcal{H}\left\{-a \mathcal{H}\left[L_{w^{\prime}}^{-1} v, \dot{w}^{\prime}\right]+a \mathcal{H}\left(a\left[L_{w^{\prime}}^{-1} v, w^{\prime \prime}\right]\right)+\mathcal{H}\left[L_{w^{\prime}}^{-1} v, \ddot{w}\right]\right. \\
& -\mathcal{H}\left(a\left[L_{w^{\prime}}^{-1} v, \dot{w}^{\prime}\right]\right)+\mu\left(v-L_{w^{\prime}}^{-1} v\right) \\
& +\frac{1}{D}\left\{\pi_{0}\left(a\left[L_{w^{\prime}}^{-1} v, w^{\prime \prime}\right]\right)-\pi_{0}\left[L_{w^{\prime}}^{-1} v, \dot{w}^{\prime}\right]\right\} \pi_{0}\left(L_{w^{\prime}} \dot{w}\right) \\
& \left.+\frac{\left(\pi_{0} L_{w^{\prime}} \dot{w}\right)^{2}}{D} \mathcal{H}\left(\frac{1}{D}\left[L_{w^{\prime}}^{-1} v, w^{\prime \prime}\right]\right)\right\},
\end{aligned}
$$

since

$\partial_{x} \mathcal{H}\left(\pi_{0}\left(D^{-1}\left[L_{w^{\prime}}^{-1} f, \dot{w}^{\prime}\right]\right) \pi_{0}\left(L_{w^{\prime}} \dot{w}\right)\right)=0$ and $\left.\pi_{0}\left(D^{-1}\left[L_{w^{\prime}}^{-1} v, w^{\prime \prime}\right]\right)\left(\pi_{0} L_{w^{\prime}} \dot{w}\right)\right)=0$.

The second observation follows because $v$ and $w$ are even in $x$, which implies that $D^{-1}\left[L_{w^{\prime}}^{-1} v, w^{\prime \prime}\right]$ is odd in $x$. From (C.1) with $u=\dot{w}^{\prime}, w^{\prime \prime}$ we observe, after integrations by parts, that

$$
\pi_{0}\left(a\left[L_{w^{\prime}}^{-1} v, w^{\prime \prime}\right]\right)-\pi_{0}\left[L_{w^{\prime}}^{-1} v, \dot{w}^{\prime}\right]=\pi_{0}\left(a_{1} v\right)
$$

where $a_{1}$ is defined in (5.17). Again from (C.1) with $u=w^{\prime \prime}$ and (3.2)

$$
\mathcal{H}\left(\frac{1}{D}\left[L_{w^{\prime}}^{-1} v, w^{\prime \prime}\right]\right)=-\mathcal{H}\left\{v\left(\frac{1}{D}\right)^{\prime}\right\}+\frac{D}{2}\left(\frac{1}{D}\right)^{\prime} \mathcal{H}\left(\frac{v}{D}\right)+\frac{v L_{w^{\prime}} w^{\prime \prime}}{D^{2}}
$$

where, as noted at the beginning of Appendix E,

$$
\left(\frac{1}{D}\right)^{\prime}=-\frac{2}{D} \mathcal{H}\left(\frac{L_{w^{\prime}} w^{\prime \prime}}{D}\right)
$$


and, from (A.1), $\pi_{0}\left(\left(L_{w^{\prime}} w^{\prime \prime}\right) / D\right)=\pi_{0}\left(w^{\prime \prime}\right)=0$. From Lemma 3.2, it follows that

$$
\left[L_{w^{\prime}}^{-1} v, u\right]=v \mathcal{H}\left(\frac{L_{w^{\prime}} u}{D}\right)-\mathcal{H}\left(\frac{v}{D}\right) L_{w^{\prime}} u
$$

and

$$
\begin{aligned}
\mathcal{H}\left(\mathcal{H}\left(\frac{v}{D}\right)\right. & \left.L_{w^{\prime}} u\right) \\
& =-\mathcal{H}\left(\frac{v}{D} \mathcal{H} L_{w^{\prime}} u\right)+\mathcal{H}\left(\frac{v}{D}\right) \mathcal{H}\left(L_{w^{\prime}} u\right)-\frac{v}{D} L_{w^{\prime}} u+\pi_{0}\left(\frac{v}{D}\right) \pi_{0}\left(L_{w^{\prime}} u\right) .
\end{aligned}
$$

Hence, from (3.7) and these observations with $u=\dot{w}^{\prime}, w^{\prime \prime}, \ddot{w}$, we finally obtain

$$
\begin{aligned}
\partial_{x} \mathcal{H} \mathcal{T}_{\mu} v= & \partial_{x} \mathcal{H}\left\{a \mathcal{H}\left(a_{1} v\right)+\mathcal{H}\left(a_{2} v\right)+a_{3} \mathcal{H}(v / D)+b v\right. \\
& \left.+\frac{1}{D}\left(\pi_{0} L_{w^{\prime}} \dot{w}\right) \pi_{0}\left(a_{1} v\right)-\frac{\left(\pi_{0} L_{w^{\prime}} \dot{w}\right)^{2}}{D} \mathcal{H}\left(v\left(\frac{1}{D}\right)^{\prime}\right)\right\}
\end{aligned}
$$

where, since $\pi_{0}\left(L_{w^{\prime}} \dot{w}^{\prime}\right)=\pi_{0}\left(a L_{w^{\prime}} w^{\prime \prime}\right)=0$ because $L_{w^{\prime}} \dot{w}^{\prime}$ and $a L_{w^{\prime}} w^{\prime \prime}$ are odd, and since

$$
\partial_{x} \mathcal{H}\left\{\pi_{0}\left(a L_{w^{\prime}} \dot{w}^{\prime}-L_{w^{\prime}} \ddot{w}\right) \pi_{0}(v / D)\right\}=0
$$

$$
\begin{aligned}
a_{1}= & a \mathcal{H}\left(L_{w^{\prime}} w^{\prime \prime} / D\right)-\mathcal{H}\left(L_{w^{\prime}} \dot{w}^{\prime} / D\right)-D^{-1} \mathcal{H}\left(L_{w^{\prime}} \dot{w}^{\prime}-a L_{w^{\prime}} w^{\prime \prime}\right), \\
a_{2}= & \mathcal{H}\left(L_{w^{\prime}} \ddot{w} / D\right)-a \mathcal{H}\left(L_{w^{\prime}} \dot{w}^{\prime} / D\right)-D^{-1} \mathcal{H}\left(a L_{w^{\prime}} \dot{w}^{\prime}-L_{w^{\prime}} \ddot{w}\right), \\
a_{3}= & a \mathcal{H}\left(L_{w^{\prime}} \dot{w}^{\prime}-a L_{w^{\prime}} w^{\prime \prime}\right)+\mathcal{H}\left(a L_{w^{\prime}} \dot{w}^{\prime}-L_{w^{\prime}} \ddot{w}\right)-\mu w^{\prime}+ \\
& +\left(\pi_{0}\left(L_{w^{\prime}} \dot{w}\right)\right)^{2}\left(\frac{1}{2 D}\right)^{\prime}, \\
b= & D^{-1}\left\{a^{2} L_{w^{\prime}} w^{\prime \prime}-2 a L_{w^{\prime}} \dot{w}^{\prime}+L_{w^{\prime}} \ddot{w}+\mu\left(D-1-\mathcal{H} w^{\prime}\right)\right\}+ \\
& +\frac{L_{w^{\prime}} w^{\prime \prime}}{D^{3}}\left(\pi_{0}\left(L_{w^{\prime}} \dot{w}\right)\right)^{2} .
\end{aligned}
$$

The estimates (5.3) follow easily from the formulae for $a$ and $b$ and from the general inequality, which is valid for any $f$ and $g$ in $H_{\text {比 }}^{l}, l \geq 2$

$$
\|f g\|_{l} \leq c_{l}\left(\|f\|_{2}\|g\|_{l}+\|f\|_{l}\|g\|_{2}\right)
$$

It remains to calculate $a_{1}, a_{2}$ and $a_{3}$.

Proposition 5.4. For any $w \in H_{\text {吅 }}^{m, e e}, m \geq 4$, we have $a \in H_{\text {响 }}^{m-1, o o}, \mathcal{F} \in$ $H_{\text {如 }}^{m-2, e e}$ and

$$
a_{1}=-a^{\prime}, \quad a_{2}=\dot{a}, \quad a_{3}=-\mathcal{H}(\mathcal{F}(w, \mu))
$$

Proof. The proof is in Appendix E. 
This completes the first part of the proof of Lemma 5.1. Now assuming that $w$ is an approximate solution at order $\varepsilon^{2}$ of (4.1), where $\mu=1+\varepsilon^{2} / 4$ (see Section 4.2), we can then compute these coefficients up to order $\varepsilon^{2}$. This is done in Appendix F, and Lemma 5.1 is proven.

Lemma 5.5. The linear operator defined in (5.2) has the function

$$
v_{0}=1+\mathcal{H} w^{\prime} \in H_{\text {如 }}^{m-1, e e}
$$

in its kernel and its range is orthogonal (in $L_{\text {如 }}^{2}$ ) to constants.

Proof. As was observed in Remark 4.1,

$$
\mathcal{F}(w+a, \mu)=\mathcal{F}(w, \mu)
$$

for any real constant $a$ and sufficiently smooth function $w$, where $\mathcal{F}$ is defined by (4.1) written as (4.2). Therefore

$$
\partial_{w} \mathcal{F}(w, \mu) a=0
$$

for a sufficiently smooth $w$ and any constant $a$. This means that $1+\mathcal{H} w^{\prime}=$ $L_{w^{\prime}}(1)$ belongs to the kernel of the linear operator defined in (5.2). The orthogonality of the range to constant functions is trivial, since the operator is a sum of derivatives $\partial_{x}$ and $\partial_{t}$. This orthogonality holds when $w \in H_{\text {响 }}^{2, e e}$.

\section{Reduction of Linearized Operator}

\subsection{Strategy}

Observe that (5.2) with $v=L_{w^{\prime}}(\delta w)$ may be written, using (5.6), as

$$
\begin{aligned}
\partial_{t}\left(\dot{v}-\partial_{x}(a v)\right)+\mathcal{H} \partial_{x}\left\{a \mathcal{H}\left(\dot{v}-\partial_{x}(a v)\right)\right\} & \\
& -\mathcal{H} \partial_{x}\{(\mu-b) v\}+\Gamma(\mathcal{F}(w, \mu), v)=f
\end{aligned}
$$

where, with $\mathcal{F}, v$ even in $x$,

$$
\begin{aligned}
\Gamma(\mathcal{F}, v)= & -\mathcal{H} \partial_{x}\{\mathcal{H}(\mathcal{F}) \mathcal{H}(v / D)\}+\mathcal{H} \partial_{x}(1 / D) \pi_{0}(\dot{v}) \int_{0}^{t} \pi_{0}(\mathcal{F}) d s \\
& -\mathcal{H} \partial_{x}\left(\frac{1}{D} \mathcal{H} \partial_{x}(v / D)\right)\left(\int_{0}^{t} \pi_{0}(\mathcal{F}) d s\right)^{2}
\end{aligned}
$$

Note that the range of the linear operator $\Gamma(\mathcal{F}, \cdot)$ is orthogonal in $L_{\text {如 }}^{2}$ to constants. Now define a new operator $\mathcal{L}(w, \mu)$ by

$$
\mathcal{L}(w, \mu)=\partial_{w} \mathcal{F}(w, \mu)-\Gamma\left(\mathcal{F}(w, \mu), L_{w^{\prime}}(\cdot)\right),
$$

and let $\Lambda(u, \varepsilon)$ be given by

$$
\Lambda(u, \varepsilon)=\mathcal{L}\left(w_{\varepsilon}^{\left(N_{0}\right)}+\varepsilon^{N_{0}} u, 1+\frac{\varepsilon^{2}}{4}\right) .
$$


The ranges of $\Lambda(u, \varepsilon)$ and $\mathcal{L}(u, \varepsilon)$ are orthogonal to constants and, from (4.12),

$$
\Phi_{u}^{\prime}(u, \varepsilon)-\Lambda(u, \varepsilon)=\Gamma\left(\mathcal{F}\left(w_{\varepsilon}^{\left(N_{0}\right)}+\varepsilon^{N_{0}} u, 1+\frac{\varepsilon^{2}}{4}\right), L_{\left\{w_{\varepsilon}^{\left(N_{0}\right)}+\varepsilon^{N_{0}} u\right\}^{\prime}}(\cdot)\right),
$$

which is zero when (4.10) is satisfied, and estimating the inverse of $\Lambda(u, \varepsilon)$ is equivalent to estimating inverses of $\mathcal{L}(w, \mu)$. This is a good place to record the obvious estimates that lead to the verification of (N.1e) when $r-\rho=3$ for $\rho \geq 1$. Suppose that $|\mu|+\|w\|_{k} \leq M_{k}, k=3,4$. Then there exist constants $c_{l}\left(M_{k}\right), l \in \mathbb{N}$, such that

$$
\left\|L_{w^{\prime}}(\delta w)\right\|_{l} \leq c_{l}\left(M_{3}\right)\left\{\|w\|_{l+1}\|\delta w\|_{2}+\|\delta w\|_{l}\right\}
$$

and hence

$$
\begin{aligned}
\|\Gamma(\mathcal{F}(w, \mu), v)\|_{l+1} \leq & c_{l}\left(M_{4}\right)\left\{\|\mathcal{F}(w, \mu)\|_{2}\|v\|_{l+3}\right. \\
& \left.+\|\mathcal{F}(w, \mu)\|_{l+2}\|v\|_{3}+\|w\|_{l+4}\|\mathcal{F}(w, \mu)\|_{2}\|v\|_{3}\right\} .
\end{aligned}
$$

Therefore

$$
\begin{aligned}
\left\|\Gamma\left(\mathcal{F}(w, \mu), L_{w^{\prime}}(\delta w)\right)\right\|_{l+1} \leq c_{l}\left(M_{4}\right)\left\{\|\mathcal{F}(w, \mu)\|_{2}\|\delta w\|_{l+3}\right. \\
\left.+\|\mathcal{F}(w, \mu)\|_{l+2}\|\delta w\|_{3} \quad+\|w\|_{l+4}\|\mathcal{F}(w, \mu)\|_{2}\|\delta w\|_{3}\right\} .
\end{aligned}
$$

To verify the hypotheses of the Nash-Moser theory we need to show that for carefully chosen positive $\varepsilon$ which can be arbitrarily small, and for $u$ sufficiently close to 0 , the equation

$$
\Lambda(u, \varepsilon) \underline{u}=\underline{f}
$$

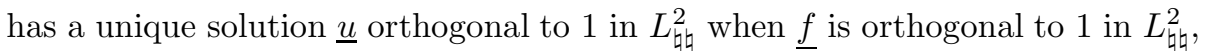
and to establish bounds on $\underline{u}$ relative to $f$ in Sobolev norms. It will suffice to prove that for carefully chosen $\mu>1$ with $\bar{\mu}$ close to 1 , and $w$ in a set determined by the proposed asymptotic form of $w$ close to zero, the equation

$$
\mathcal{L}(w, \mu) \underline{u}=\underline{f}
$$

has a unique solution $\underline{u}$ orthogonal to 1 when $f$ is orthogonal to 1 , with suitable bounds on $\underline{u}$ in terms of $\underline{f}$. Note from (6.1) that

$$
\begin{gathered}
\mathcal{L}(w, \mu)=\mathcal{A}(w, \mu) \circ L_{w^{\prime}} \text { where } \\
\mathcal{A}(w, \mu) v=\partial_{t}\left(\dot{v}-\partial_{x}(a v)\right)+\mathcal{H} \partial_{x}\left\{a \mathcal{H}\left(\dot{v}-\partial_{x}(a v)\right)\right\}-\mathcal{H} \partial_{x}\{(\mu-b) v\} .
\end{gathered}
$$

The aim is to prove Theorem 8.5. In what follows we show that the operator $\mathcal{A}(w, \mu)$ has one-dimensional kernel, spanned by $v^{*}$, say, where $v^{*}$ is close to 1 , that its range is the space orthogonal to 1 , and establish good estimates on its inverse restricted to these codimension- 1 spaces. To see that this is sufficient, suppose that $\underline{f}$ is orthogonal to 1 and that $\mathcal{A}(w, \mu) \underline{v}=\underline{f}$ where $\underline{v}$ is orthogonal to $v^{*}$. Let $\underline{u}=L_{w^{\prime}}^{-1}\left(\underline{v}+\alpha v^{*}\right)$. Then

$$
\mathcal{L}(w, \mu) \underline{u}=\underline{f}
$$


and $\underline{u}$ is orthogonal to 1 if and only if

$$
\int_{0}^{2 \pi} \int_{0}^{2 \pi} L_{w^{\prime}}^{-1}\left(\underline{v}+\alpha v^{*}\right) d x d t=0,
$$

which uniquely determines $\alpha$, because $v^{*}$ is close to 1 and $L_{w^{\prime}}$ is close to the identity. This solution is clearly unique in the class of functions orthogonal to 1 , and estimates on $\underline{u}$ in terms of $f$ follow once similar estimates of $\underline{v}$ in terms of $f$ have been established.

\subsection{Change of coordinates and smoothing operators}

Thus we have reduced the problem to one of estimate the inverse of $\mathcal{A}(w, \mu)$. To do so we seek a change of coordinates to remove terms which contain second order derivatives in $x x$ and in $x t$ in $\mathcal{A}(w, \mu)$. Such a change of coordinates is defined by

$$
y=x+d(x, t) \stackrel{\text { def }}{=} \mathcal{U}_{t}(x),
$$

where $d$ satisfies the linear PDE

$$
\begin{aligned}
\partial_{t} d & =a\left(1+\partial_{x} d\right) \\
\left.d\right|_{t=0} & =0 .
\end{aligned}
$$

It follows from the method of characteristics, because of the periodicity and oddness of $a(x, t)$, that there exists a solution $d$ in $C^{k}$ when $a \in C^{k}$. In particular if $w \in H_{\text {吅 }}^{m, e e}$ then $a \in H_{\text {吅 }}^{m-1, o o} \subset C_{\text {吅 }}^{m-3, o o}$ and the existence of a solution $d \in C_{\text {吅 }}^{m-3, o e} \subset H_{\text {唢 }}^{m-3, o e}$ follows. Moreover, equating of powers of $\varepsilon$ yields that in $C_{\text {她 }}^{m-3, o e}$, as $\varepsilon \rightarrow 0$,

$$
d(x, t)=2 \varepsilon(1-\cos t) \sin x+\varepsilon^{2} \sin 2 x(1-2 \cos t+\cos 2 t)+O\left(\varepsilon^{3}\right) .
$$

For the method of characteristics and estimates of $d$ and $\tilde{d}$, see Appendix G.

Remark 6.1. In principle, the initial value $\left.d\right|_{t=0}$ might be chosen as an arbitrary odd periodic function of $x$. We choose zero initial data for the moment, but a further change of variables will amount to re-introducing a non-zero initial value for $d(x, t)$ (see $d_{1}(x, t)$ in $\left.(7.12)\right)$.

From now on a tilde $(\sim)$ will indicate a function of $(x, t)$ expressed as a function of $(y, t)$ via the formula

$$
\tilde{u}(y, t)=u\left(\mathcal{U}_{t}^{-1}(y), t\right),
$$

and a hat $\left({ }^{\wedge}\right)$ will indicate a function of $(y, t)$ expressed as a function of $(x, t)$ via the formula

$$
\widehat{v}(x, t)=v\left(\mathcal{U}_{t}(x), t\right),
$$




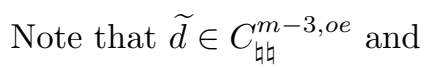

$$
\begin{aligned}
\mathcal{U}_{t}(x)=x+d(x, t) & =x+\tilde{d}\left(\mathcal{U}_{t}(x), t\right), \\
\mathcal{U}_{t}^{-1}(y) & =y-\widetilde{d}(y, t), \\
1+\partial_{x} d\left(\mathcal{U}_{t}^{-1}(y), t\right) & =\frac{1}{1-\partial_{y} \widetilde{d}(y, t)}, \\
\left\{\partial_{t} v-\partial_{x}(a v)\right\}\left(\mathcal{U}_{t}^{-1}(y), t\right) & =\frac{\partial_{t}(p \widetilde{v})(y, t)}{p(y, t)},
\end{aligned}
$$

where

$$
p(y, t)=\exp \left\{-\int_{0}^{t} \partial_{x} a\left(\mathcal{U}_{s}^{-1}(y), s\right) d s\right\} .
$$

Proposition 6.2. For any $w \in H_{\text {仲 }}^{m, e e}$ and $m \geq 4, p \in H_{\text {如 }}^{m-3, e e}, \widetilde{d} \in C_{\text {仲 }}^{m-3, o e}$, and

$$
p(y, t)=1-\partial_{y} \widetilde{d}(y, t) .
$$

Proof. By construction,

$$
\frac{\partial_{t} p(y, t)}{p(y, t)}=-\partial_{x} a\left(\mathcal{U}_{t}^{-1}(y), t\right)=\frac{-\partial_{y} \widetilde{a}(y, t)}{1-\partial_{y} \widetilde{d}(y, t)} .
$$

Also, from the definition of $\widetilde{d}$ and (6.9),

$$
\left(\partial_{t} \widetilde{d}\right)\left(\mathcal{U}_{t}(x), t\right)+\left(\partial_{y} \widetilde{d}\right)\left(\mathcal{U}_{t}(x), t\right) \partial_{t} d(x, t)=\partial_{t} d(x, t) .
$$

Therefore we obtain from (6.10) and (6.14) that $\partial_{t} \widetilde{d}=\widetilde{a}$, and so that

$$
\frac{\partial_{t}\left(1-\partial_{y} \widetilde{d}\right)}{1-\partial_{y} \widetilde{d}}=\frac{-\partial_{y} \widetilde{a}}{1-\partial_{y} \widetilde{d}}=\frac{\partial_{t} p}{p}
$$

Since $\left.p\right|_{t=0}=\left.\left(1-\partial_{y} \widetilde{d}\right)\right|_{t=0}=1$, the result of the proposition follows from the uniqueness theory of initial-value problems.

To continue the study of the linear operator (5.2), or equivalently of (6.1) we examine the smoothing properties of two families of operators $\mathcal{S}_{\omega}$ and $\mathcal{S}$.

Lemma 6.3. For a smooth $2 \pi$-periodic function $\omega$, define the linear operator $\mathcal{S}_{\omega}$ on $L_{\natural}^{2}$ by

$$
\mathcal{S}_{\omega} f=\mathcal{H}(\omega f)-\omega \mathcal{H} f, \quad f \in L_{\sharp}^{2} .
$$

Then, for $\omega \in H_{\natural}^{m}, \mathcal{S}_{\omega}$ is bounded from $L_{\natural}^{2}$ into $H_{\natural}^{m-2}$ with, for any $m \geq 2$,

$$
\left\|\mathcal{S}_{\omega}\right\|_{\mathcal{L}\left(L_{\natural}^{2}, H_{\natural}^{m-2}\right)} \leq c_{m}\|\omega\|_{H_{\natural}^{m}} .
$$

Proof. The proof is in Appendix H. 
Q1D Operators. In what follows the notion of a Q1D (quasi-1-dimensional) operator is from [22, Definition 7.6]. Recall the notation of the Fourier transform from (4.3). For any real $\alpha, \beta$ denote by $G_{\alpha, \beta}$ the operator on $H^{s}$ defined by

$$
\mathfrak{F}\left(G_{\alpha, \beta} u\right)(m, n)=\mathfrak{F}(m, n)(1+|n|)^{-\alpha}(1+|m|)^{\beta}, \quad(m, n) \in \mathbb{Z}^{2} .
$$

Definition 6.4. For any non-negative exponents $\alpha, \beta$, a linear operator $R$ is quasi-one-dimensional (Q1D) of order $(\alpha, \beta)$ on $H^{s}$ if

$$
\|R\|\left\|_{\alpha, \beta, s}:=\sup _{\|u\|_{s}=1}\right\| G_{\alpha, \beta} R u \|_{s}<\infty .
$$

It follows from this definition that if $R$ is a Q1D operator of order $(\alpha, \beta)$ in $H^{s}$ then, for any $\alpha^{\prime} \geq \alpha$ and $\beta^{\prime} \leq \beta$, the operator $R$ has order $\left(\alpha^{\prime}, \beta^{\prime}\right)$ and $\||| R \mid\|_{\alpha^{\prime}, \beta^{\prime}, s} \leq\|\| R \|_{\alpha, \beta, s}$. Note that ||$\left|D_{1}^{p} D_{2}^{q} R\left\|_{\alpha+p, \beta-q, s} \leq\right\|\right| \mid R \|_{\alpha, \beta, s}$, for any $p, q \geq 0$. Roughly speaking, a Q1D operator of order $(\alpha, \beta)$ can lose up to $\alpha$ derivatives in $t$ while gaining $\beta$ derivatives in $x$. Examples of Q1D operators are the identity $I$ and the Hilbert transform $\mathcal{H}$, which are Q1D of order $(\alpha, 0)$ for any $\alpha \geq 0$. Also [22, Proposition 7.8] gives examples of Q1D integral operators.

Lemma 6.5. If $\omega \in C_{\text {吅 }}^{m}, m \geq 0$, then the linear operator $\mathcal{S}_{\omega}$, defined by

$$
\mathcal{S}_{\omega} f=\mathcal{H}(\omega f)-\omega \mathcal{H} f
$$

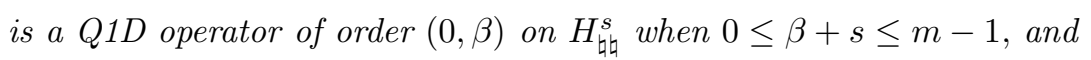

$$
\left\|G_{0, \beta} \mathcal{S}_{\omega} f\right\|_{s} \leq c_{\beta, s}\left(\|\omega\|_{C^{\beta+s+1}}\|f\|_{0}+\|\omega\|_{C^{\beta+1}}\|f\|_{s}\right) .
$$

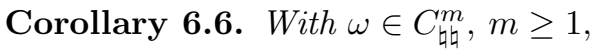

$$
\mathcal{S}_{\omega}\left(\partial_{t} f\right)=\partial_{t}\left(\mathcal{S}_{\omega} f\right)-\mathcal{S}_{\dot{\omega}} f \quad \text { and } \mathcal{S}_{\omega}\left(\partial_{x} f\right)=\partial_{x}\left(\mathcal{S}_{\omega} f\right)-\mathcal{S}_{\omega^{\prime}} f,
$$

where

$$
\left\|G_{0, \beta} \mathcal{S}_{\dot{\omega}} f\right\|_{s}+\left\|G_{0, \beta} \mathcal{S}_{\omega}\left(\partial_{x} f\right)\right\|_{s} \leq c_{\beta, s}\left(\|\omega\|_{C^{\beta+s+2}}\|f\|_{0}+\|\omega\|_{C^{\beta+2}}\|f\|_{s}\right) .
$$

Corollary 6.7. Let $w \in H_{\text {吅 }}^{m}, m \geq 4,\|w\|_{4} \leq M_{4}$ and $\omega \in C_{\text {吅 }}^{l}$ be such that

$$
\|\omega\|_{C^{l}} \leq c_{l}\left(M_{4}\right)\|w\|_{l+r}, \quad l \geq 0, \quad r \geq 3 .
$$

Then

$$
\left\|G_{0, \beta}\left(\mathcal{S}_{\omega} f\right) \sim\right\|_{s} \leq c_{\beta, s}\left(M_{4}\right)\left(\|w\|_{\beta+s+r+1}\|f\|_{0}+\|w\|_{\beta+r+1}\|f\|_{s}\right)
$$

for $0 \leq \beta+s \leq m-r-1$ and, for $0 \leq \beta+s \leq m-r-2$,

$$
\begin{aligned}
\left\|G_{0, \beta}\left(\mathcal{S}_{\dot{\omega}} f\right)^{\sim}\right\|_{s} & \leq c_{\beta, s}\left(M_{4}\right)\left(\|w\|_{\beta+s+r+2}\|f\|_{0}+\|w\|_{\beta+r+2}\|f\|_{s}\right), \\
\left\|G_{0, \beta}\left(\mathcal{S}_{\omega} \partial_{x} f\right)^{\sim}\right\|_{s} & \leq c_{\beta, s}\left(M_{4}\right)\left(\|w\|_{\beta+s+r+2}\|f\|_{0}+\|w\|_{\beta+r+2}\|f\|_{s}\right) .
\end{aligned}
$$

Note that $r=3$ when $\omega=a$ and $r=4$ when $\omega=q$ or $\omega=\dot{a}$. 
Proof. The proof is in Appendix H.

Lemma 6.8. Suppose that $w \in H_{\natural \natural}^{m, e e} \backslash\{0\}, m \geq 5$ and $\|w\|_{4} \leq M_{4}$ sufficiently small. Then $\widetilde{d}$ and $d$ are in $C_{\text {如 }}^{m-3, o e}$ and the linear operator $\mathcal{S}$ defined for $u \in L_{\text {如 }}^{2}$ by

$$
\mathcal{S} u(y, t)=(\mathcal{H} \widehat{u})^{\sim}(y, t)-(\mathcal{H} u)(y, t)
$$

satisfies the estimate

$$
\left\|G_{0, \beta} \mathcal{S} u\right\|_{s} \leq c_{\beta, s}\left(M_{4}\right)\left(\|w\|_{\beta+s+5}\|u\|_{0}+\|w\|_{\beta+5}\|u\|_{s}\right) .
$$

Hence $\mathcal{S}$ is a $Q 1 D$ operator of order $(0, \beta)$ on $H_{\text {如 }}^{s}$ if $0 \leq \beta+s \leq m-5$.

Remark. Observe that these operators are smoothing in $x$.

Proof. The proof is in Appendix I.

Remark. When $w \in H_{\natural \natural}^{m, e e}$, then $d \in C^{m-3}$ and, by [18, Theorem 3.1.5], the change of coordinate $x \mapsto y=\mathcal{U}_{t}(x)$ defined by (6.9), is such that if $f \in H_{\natural \natural}^{k, e e}$, $k \leq m-3$, then $\tilde{f} \in H_{\natural \natural}^{k, e e}$ and the linear map $f \mapsto \tilde{f}$ is bounded. Similarly, $g \rightarrow \widehat{g}$ is bounded in the same setting. Therefore both are Q1D operators on $H_{\text {如 }}^{s}$ of order $(\alpha, 0)$ for all $\alpha \geq 0$ and all $0 \leq s \leq m-3$. When $q \in C_{\text {t女 }}^{m-4}$ the multiplication operator $\varphi \mapsto q \varphi$ is a Q1D operator of order $(\alpha, 0)$ for all $\alpha \geq 0$ on $H^{s}$ if $0 \leq s \leq m-4$. See also Appendix G for estimates of changing variables.

\subsection{New linearized equation}

Now we show how the change of coordinates $(x, t) \mapsto(y, t)=\left(\mathcal{U}_{t}(x), t\right)$ leads to a linear equation in which the $x x$ and $x t$ derivatives are absent but terms involving first order $t$-derivative remain. The next theorem develops the result of Lemma 5.1.

Theorem 6.9. For $w \in H_{\text {姉 }}^{m, e e} \backslash\{0\}, m \geq 6$, let $\mathcal{A}(w, \mu)$ be defined by (6.8). Suppose that $\mathcal{A}(w, \mu) v=f$ and let

$$
\varphi(y, t)=\tilde{v}(y, t) p(y, t)
$$

where $p \in C_{\text {吼 }}^{m-4, e e}$ is defined by (6.16). Then $\varphi \in H_{\text {㕷 }}^{k, e e}, k \leq m-3$, satisfies the linear equation

$$
\partial_{t t} \varphi-\partial_{y}\{q \mathcal{H} \varphi\}+\mathcal{G}(\varphi)=p \tilde{f}
$$

where

$$
\begin{gathered}
q=(\mu-\widetilde{b}) / p \in C_{\text {如 }}^{m-4, e e}, \\
\mathcal{G}(\varphi)=-\partial_{y}\left\{\mathcal{S}(q \varphi)+\mathcal{S}_{q} \varphi+\left(\mathcal{H}_{a}\left(\widehat{\partial_{t} \varphi / p}\right)\right)^{\sim}\right\} .
\end{gathered}
$$


When $\|w\|_{4} \leq M_{4}$, the coefficients $q$ above and $p$ from Proposition 6.2 satisfy the estimates

$$
\|p-1\|_{C^{l}}+\|q-\mu\|_{C^{l}} \leq c_{l}\left(M_{4}\right)\|w\|_{l+4},
$$

and the operator $\mathcal{G}$ is $Q 1 D$ on $H^{s}$ of order $(1, \beta), 0 \leq \beta+s \leq m-6$ with

$$
\left\|G_{1, \beta} \mathcal{G}(\varphi)\right\|_{s} \leq c_{\beta, s}\left(M_{5}\right)\left\{\|w\|_{\beta+s+6}\|\varphi\|_{0}+\|w\|_{\beta+6}\|\varphi\|_{s}\right\} .
$$

Moreover, the range of the linear operator defined by (6.17) is orthogonal in $L_{\text {如 }}^{2}$ to constants.

Proof. The linear equation $\mathcal{A}(w, \mu) v=f$ can be re-written in terms of the smoothing operator $\mathcal{S}_{a}$ defined in Lemma 6.8 as

$$
f=\left(\partial_{t}-\partial_{x} a\right)^{2} v-\mathcal{H} \partial_{x}\{(\mu-b) v\}-\mathcal{H} \partial_{x} \mathcal{S}_{a}\left(\partial_{t} v-\partial_{x}(a v)\right) .
$$

Now using the change of coordinate (6.9) with (6.15) and the identity

$$
\left(\partial_{x} f\right)\left(\mathcal{U}_{t}^{-1}(y), t\right)=\frac{\partial_{y} \widetilde{f}(y, t)}{1-\partial_{y} \widetilde{d}(y, t)}=\frac{\partial_{y} \widetilde{f}(y, t)}{p(y, t)}, \text { (from Proposition 6.2) }
$$

equation (6.21) becomes

$$
\tilde{f}=\frac{1}{p} \partial_{t t} \varphi-\frac{1}{p} \partial_{y}\left(\mathcal{H}\left(\{(\mu-\tilde{b}) \varphi / p\}^{\wedge}\right)\right)^{\sim}-\frac{1}{p} \partial_{y}\left(\mathcal{H}\left(\mathcal{S}_{a}\left(\widehat{\partial_{t} \varphi / p}\right)\right)\right)^{\sim} .
$$

Now using the smoothing operator $\mathcal{S}$ defined in Lemma 6.8, we find

$$
\left(\mathcal{H}\left(\{(\mu-\tilde{b}) \varphi / p\}^{\wedge}\right)\right)^{\sim}=\mathcal{H}(q \varphi)+\mathcal{S}(q \varphi)=q \mathcal{H} \varphi+\mathcal{S}_{q} \varphi+\mathcal{S}(q \varphi)
$$

which leads to (6.17). Estimate (6.20) holds because Proposition 6.2 and definition (6.18), with the estimates (5.3) and (G.3), show that

$$
\|p-1\|_{C^{l}}+\|q-\mu\|_{C^{l}} \leq\left\|\frac{\partial_{y} \tilde{d}}{1-\partial_{y} \tilde{d}}\right\|_{C^{l}}+\left\|\frac{\tilde{b}}{p}\right\|_{C^{l}} \leq c_{l}\left(M_{4}\right)\|w\|_{l+4} .
$$

To estimate $\mathcal{G}$ note that

$$
\begin{aligned}
\|q \varphi\|_{s} & \leq c_{s}\left(\|q\|_{C^{s}}\|\varphi\|_{0}+\|q\|_{C^{0}}\|\varphi\|_{s}\right) \\
& \leq c_{s}\left(M_{4}\right)\left(\|w\|_{s+4}\|\varphi\|_{0}+\|w\|_{4}\|\varphi\|_{s}\right) .
\end{aligned}
$$

Now by Lemma 6.8 if $0 \leq \beta+s \leq m-6$,

$$
\begin{aligned}
\left\|G_{1, \beta} \partial_{y} \mathcal{S}(q \varphi)\right\|_{s} \leq & \left\|G_{0, \beta+1} \mathcal{S}(q \varphi)\right\|_{s} \\
\leq & c_{\beta+1, s}\left(M_{4}\right)\left(\|w\|_{\beta+s+6}\|q \varphi\|_{0}+\|w\|_{\beta+6}\|q \varphi\|_{s}\right) \\
\leq & c_{\beta+1, s}\left(M_{4}\right)\left(\|w\|_{\beta+s+6}\|\varphi\|_{0}\right. \\
& \quad+\|w\|_{\beta+6}\left(\|w\|_{s+4}\|\varphi\|_{0}+\|w\|_{4}\|\varphi\|_{s}\right) \\
\leq & c_{\beta+1, s}\left(M_{4}\right)\left(\|w\|_{\beta+s+6}\|\varphi\|_{0}+\|w\|_{\beta+6}\|\varphi\|_{s}\right)
\end{aligned}
$$


(where the constant changes from line to line), since for

$$
0 \leq \alpha_{1} \leq \alpha_{2} \leq \beta_{2} \leq \beta_{1}, \quad \alpha_{1}+\beta_{1}=\alpha_{2}+\beta_{2}
$$

we have

$$
\|f\|_{\alpha_{2}}\|f\|_{\beta_{2}} \leq c|| f\left\|_{\alpha_{1}}\right\| f \|_{\beta_{1}}
$$

which results directly from the classical interpolation inequality

$$
\|f\|_{\lambda \alpha+(1-\lambda) \beta} \leq c|| f\left\|_{\alpha}^{\lambda}\right\| f \|_{\beta}^{1-\lambda} \text {. }
$$

Similarly, by Lemma 6.5 ,

$$
\begin{aligned}
\left\|G_{1, \beta} \partial_{y} \mathcal{S}_{q} \varphi\right\|_{s} & \leq\left\|G_{0, \beta+1} \mathcal{S}_{q} \varphi\right\|_{s} \\
& \leq c_{\beta+1, s}\left(\|q\|_{C^{\beta+s+2}}\|\varphi\|_{0}+\|q\|_{C^{\beta+2}}\|\varphi\|_{s}\right) \\
& \leq c_{\beta+1, s}\left(M_{4}\right)\left(\|w\|_{\beta+s+6}\|\varphi\|_{0}+\|w\|_{\beta+6}\|\varphi\|_{s}\right) .
\end{aligned}
$$

By (6.15) and Lemma 6.5,

$$
\begin{aligned}
& \left(\mathcal{H} \mathcal{S}_{a}\left(\widehat{\partial_{t} \varphi / p}\right)\right)^{\sim}=\left(\mathcal{H} \mathcal{S}_{a}\left(\partial_{t} v-\partial_{x}(a v)\right)\right)^{\sim} \\
& =\left(\partial_{t} \mathcal{H} \mathcal{S}_{a} v\right)^{\sim}-\left(\mathcal{H}\left(\mathcal{S}_{a} \dot{a} v\right)\right)^{\sim}-\left(\mathcal{H}\left(\mathcal{S}_{a} \partial_{x}(a v)\right)\right)^{\sim} \\
& =\partial_{t}\left(\left(\mathcal{H} \mathcal{S}_{a} v\right)^{\sim}\right)-\tilde{a}\left(\partial_{x}\left(\mathcal{H} \mathcal{S}_{a} v\right)\right)^{\sim}-\left(\mathcal{H}\left(\mathcal{S}_{\dot{a}} v\right)\right)^{\sim}-\left(\mathcal{H}\left(\mathcal{S}_{a} \partial_{x}(a v)\right)\right)^{\sim} \\
& =\mathcal{H}\left(\partial_{t}\left(\mathcal{S}_{a} v\right)^{\sim}\right)-\tilde{a} \mathcal{H}\left(\left(\partial_{x} \mathcal{S}_{a} v\right)^{\sim}\right)-\mathcal{H}\left(\left(\mathcal{S}_{\dot{a}} v\right)^{\sim}\right)-\mathcal{H}\left(\left(\mathcal{S}_{a} \partial_{x}(a v)\right)^{\sim}\right) \\
& \quad+\partial_{t} \mathcal{S}\left(\left(\mathcal{S}_{a} v\right)^{\sim}\right)-\tilde{a} \mathcal{S}\left(\left(\partial_{x} \mathcal{S}_{a} v\right)^{\sim}\right)-\mathcal{S}\left(\left(\mathcal{S}_{\dot{a}} v\right)^{\sim}\right)-\mathcal{S}\left(\left(\mathcal{S}_{a} \partial_{x}(a v)\right)^{\sim}\right) .
\end{aligned}
$$

By Lemma 6.8 we have

$$
\begin{aligned}
\left\|G_{1, \beta} \mathcal{S}\left(\widetilde{\mathcal{S}_{\dot{a}} v}\right)\right\|_{s} \leq\left\|G_{0, \beta} \mathcal{S}\left(\widetilde{\mathcal{S}_{\dot{a}} v}\right)\right\|_{s} & \quad \leq c_{\beta, s}\left(M_{4}\right)\left(\|w\|_{\beta+s+5}\left\|\widetilde{\mathcal{S}_{\dot{a}} v}\right\|_{0}+\|w\|_{\beta+5}\left\|\widetilde{\mathcal{S}_{\dot{a}} v}\right\|_{s}\right)
\end{aligned}
$$

and then, by Corollary 6.7 (with $\beta=0$ ),

$$
\begin{aligned}
\left\|G_{1, \beta} \mathcal{S}\left(\widetilde{\mathcal{S}_{\dot{a}} v}\right)\right\|_{s} & \leq c_{\beta, s}\left(M_{5}\right)\left\{\|w\|_{\beta+s+5}\|v\|_{0}+\|w\|_{\beta+5}\left(\|w\|_{s+5}\|v\|_{0}+\|v\|_{s}\right)\right\} \\
& \leq c_{\beta, s}\left(M_{5}\right)\left\{\|w\|_{\beta+s+5}\|v\|_{0}+\|w\|_{\beta+5}\|v\|_{s}\right\}
\end{aligned}
$$

since

$$
\|w\|_{\beta+5}\|w\|_{s+5} \leq c_{\beta, s}\|w\|_{\beta+s+5}\|w\|_{5} .
$$

Other terms involving the operator $\mathcal{S}$ may be treated similarly, using the fact that $\|\tilde{a}\|_{C^{s}} \leq c_{s}\left(M_{4}\right)\|w\|_{s+3}$ (from Lemma G.1 and (G.3)) and Lemma G.3. The terms involving $\mathcal{H}$ are estimated using the fact that

$$
\left\|G_{\alpha, \beta} \mathcal{H} u\right\|_{s}=\left\|G_{\alpha, \beta} u\right\|_{s}
$$

with Corollary 6.7 and Lemma G.3. Next recall that $v=\widehat{\varphi / p}$ and hence, by Lemma G.3 and (G.3),

$$
\|v\|_{s} \leq c_{s}\left(M_{4}\right)\left(\|\varphi\|_{s}+\|w\|_{s+4}\|\varphi\|_{0}\right)
$$


which implies that

$$
\left\|G_{1, \beta}\left(\mathcal{H} \mathcal{S}_{a}\left(\widehat{\partial_{t} \varphi / p}\right)\right)^{\sim}\right\|_{s} \leq c_{\beta, s}\left(M_{5}\right)\left\{\|w\|_{\beta+s+5}\|\varphi\|_{0}+\|w\|_{\beta+5}\|\varphi\|_{s}\right\} .
$$

These estimates yield the required estimate on $\mathcal{G}$. When it has been noted that the range of our operator is orthogonal to constants, the proof is complete.

Corollary 6.10. If $w \in H_{\natural \natural}^{m, e e}$ in Theorem 6.9 is the approximate solution $w_{\varepsilon}^{(N)}$ of (4.1) given by Lemma 4.5 with $N \geq 2$, then, in the appropriate spaces (see Theorem 6.9),

$$
\begin{aligned}
q & =1+2 \varepsilon \cos y+\varepsilon^{2}\left(\frac{9}{4}-\cos 2 t-2 \cos 2 y\right)+O\left(\varepsilon^{3}\right), \\
p & =1-2 \varepsilon(1-\cos t) \cos y+4 \varepsilon^{2}(1-\cos t) \cos 2 y+O\left(\varepsilon^{3}\right), \\
\mathcal{G} \varphi & =\varepsilon \mathcal{G}^{(1)} \varphi+\varepsilon^{2} \mathcal{G}^{(2)} \varphi+O\left(\varepsilon^{3}\|\varphi\|\right),
\end{aligned}
$$

where

$$
\begin{aligned}
\mathcal{G}^{(1)} \varphi= & 2 \cos y\left\{(\sin t) \pi_{0}\left(\partial_{t} \varphi\right)+\pi_{0} \varphi\right\}, \\
\mathcal{G}^{(2)} \varphi= & -4(\sin t \cos 2 y) \pi_{0}\left(\partial_{t} \varphi\right)-2(\sin 2 t \cos y) \pi_{0}\left(\partial_{t} \varphi \cos y\right) \\
& -4 \cos 2 y \pi_{0} \varphi-\cos y(3+\cos 2 t) \pi_{0}(\varphi \cos y) .
\end{aligned}
$$

Proof. The proof is in Appendix J.

\section{Normal Form of the Linearized Operator}

In this section we make a succession of changes of variables which transform the linear equation (6.17) into a simpler one (Theorem 7.5) in which the main part has constant coefficients and the remaining term is a Q1D operator $[22$, Section 7.2]. When this is done, the linear operator can be inverted provided certain diophantine conditions are satisfied by two of the coefficients in the new equation.

\subsection{Simplification of (6.17)}

The next change of variables is designed to produce a new linear equation with the same form as (6.17), but with a constant coefficient instead of $q(y, t)$. The idea is to change the independent variables with a diffeomorphism of the torus $\mathbb{T}^{2}=(\mathbb{R} / 2 \pi \mathbb{Z})^{2}$ in the form

$$
\xi=y+d_{0}(y), \quad \tau=t+e_{0}(y, t),
$$

where $d_{0} \in C_{\natural}^{m-3, o}, e_{0} \in C_{\natural \natural}^{m-4, e o}$ have norms of order $\varepsilon$ when $w=w_{\varepsilon}^{(N)}$ is given by Lemma 4.5. Denote this change of coordinates by

$$
(\xi, \tau)=Q(y, t)
$$


and introduce a new dependent variable

$$
\theta(\xi, \tau)=\varphi\left(Q^{-1}(\xi, \tau)\right)
$$

We will need a new smoothing operator, $\mathcal{S}_{(0)}$, defined for $\theta \in L_{\text {吅 }}^{2}$ by

$$
\mathcal{S}_{(0)} \theta=(\mathcal{H}(\theta \circ Q)) \circ Q^{-1}-\mathcal{H} \theta \text {. }
$$

The hypotheses for the next observation are motivated by estimate (7.10) which follows once $e_{0}$ and $d_{0}$ have been identified.

Lemma 7.1. Suppose $\left\|d_{0}\right\|_{C^{m-3}}+\left\|e_{0}\right\|_{C^{m-4}} \leq c_{m}\left(M_{6}\right)\|w\|_{m}$ where $\|w\|_{6} \leq M_{6}$. Then for a possibly different constant constant $c_{m}\left(M_{6}\right)$,

$$
\begin{aligned}
\left\|\mathcal{S}_{(0)} \theta\right\|_{s} & \leq c_{m}\left(M_{6}\right)\left\{\|\theta\|_{s+1}+\|w\|_{s+6}\|\theta\|_{0}\right\}, \quad s+6 \leq m, \\
\left\|G_{\beta+1, \beta} \mathcal{S}_{(0)} \theta\right\|_{s} & \leq c_{m}\left(M_{6}\right)\left\{\|w\|_{\beta+6}\|\theta\|_{s}+\|w\|_{\beta+s+6}\|\theta\|_{0}\right\}, \quad \beta+s+6 \leq m .
\end{aligned}
$$

Hence $\mathcal{S}_{(0)}$ is a $Q 1 D$ operator of order $(\beta+1, \beta)$ on $H_{\text {如 }}^{s}, 0 \leq \beta+s+6 \leq s$.

Proof. The proof is in Appendix K.

The next result concerns a further change of variables in (6.17).

Lemma 7.2. Let $\mathcal{A}(w, \mu), v, f$ and $\varphi$ be as in Theorem 6.9. Then there is a diffeomorphism $Q$ of the torus $\mathbb{T}^{2}$, determined by $(w, \mu)$ of the form (7.1) in which $d_{0} \in C_{\natural}^{m-3, o}, e_{0} \in C_{\text {七七 }}^{m-4, e o}$, such that $\theta$ defined in (7.2) satisfies the linear equation

$$
\partial_{\tau \tau} \theta-\left(1+\beta^{(0)}\right) \mathcal{H} \partial_{\xi} \theta+(\gamma+\delta \mathcal{H}) \partial_{\tau} \theta+\alpha \mathcal{H} \theta+\mathcal{G}_{0} \theta=g .
$$

Here $\beta^{(0)}$ is a constant, the coefficient functions are given by

$$
\begin{aligned}
\gamma & =\left\{\frac{\partial_{t t} e_{0}}{\left(1+\partial_{t} e_{0}\right)^{2}}\right\} \circ Q^{-1} \in C_{\text {仲 }}^{m-5, e o}, \\
\delta & =-\left(1+\beta^{(0)}\right) \delta_{0}, \quad \delta_{0}=\left(\frac{\partial_{y} e_{0}}{1+\partial_{y} d_{0}}\right) \circ Q^{-1} \in C_{\text {仲 }}^{m-5, o o}, \\
\alpha & =\left(\frac{-\partial_{y} q}{\left(1+\partial_{t} e_{0}\right)^{2}}\right) \circ Q^{-1} \in C_{\text {吅 }}^{m-5, o e}, \\
g & =\left(\frac{p \tilde{f}}{\left(1+\partial_{t} e_{0}\right)^{2}}\right) \circ Q^{-1}
\end{aligned}
$$

and the linear operator $\mathcal{G}_{0}$ on $H_{\text {如 }}^{k}, k \leq m-7$, given by

$$
\begin{aligned}
\mathcal{G}_{0}(\theta)=-\left(1+\beta^{(0)}\right)\left\{\partial_{\xi} \mathcal{S}_{(0)} \theta+\delta_{0} \partial_{\tau} \mathcal{S}_{(0)} \theta\right\} & +\alpha \mathcal{S}_{(0)} \theta \\
& +\left(\left(1+\partial_{t} e_{0}\right)^{-2}\{\mathcal{G}(\theta \circ Q)\}\right) \circ Q^{-1}
\end{aligned}
$$


is $Q 1 D$ of order $(\beta+2, \beta)$ on $H^{s}, 0 \leq \beta+s \leq m-7$ and for $\beta \leq 3$

$$
\left\|G_{\beta+2, \beta} \mathcal{G}_{0}(\theta)\right\| s \leq c_{\beta, s}\left(M_{6}\right)\left(\|w\|_{\beta+s+7}\|\theta\|_{0}+\|w\|_{\beta+7}\|\theta\|_{s}\right),
$$

while for $\beta \geq 3,0 \leq 2 \beta+s \leq m-4$,

$$
\left\|G_{\beta+2, \beta} \mathcal{G}_{0}(\theta)\right\| s \leq c_{\beta, s}\left(M_{6}\right)\left(\|w\|_{2 \beta+s+4}\|\theta\|_{0}+\|w\|_{2 \beta+4}\|\theta\|_{s}\right) .
$$

Moreover,

$$
\theta=\varphi \circ Q^{-1}=(p \tilde{v}) \circ Q^{-1}=\left(\frac{L_{w^{\prime}} \delta w}{1+d^{\prime}}\right) \circ \widehat{Q}^{-1}
$$

where, following the notation of Section 6.2, $\widehat{Q}(x, t)=Q\left(\mathcal{U}_{t}(x), t\right)$. The function

$$
\left(\frac{\left(1+\partial_{t} e_{0}\right)}{1+\partial_{y} d_{0}}\right) \circ Q^{-1}
$$

is orthogonal in $L_{\text {如 }}^{2}$ to the range of the linear operator defined by (7.5).

Proof. First note that

$$
\begin{aligned}
\partial_{y} \varphi & =\left(1+\partial_{y} d_{0}\right)\left(\partial_{\xi} \theta \circ Q\right)+\partial_{y} e_{0}\left(\partial_{\tau} \theta \circ Q\right), \\
\partial_{t t} \varphi & =\left(1+\partial_{t} e_{0}\right)^{2}\left(\partial_{\tau \tau} \theta \circ Q\right)+\partial_{t t} e_{0}\left(\partial_{\tau} \theta \circ Q\right)
\end{aligned}
$$

and, in the new variables, $\partial_{y}\{q \mathcal{H} \varphi\}=\left\{\partial_{y}[q \mathcal{H}(\theta \circ Q)]\right\} \circ Q^{-1}$ where

$\left(\partial_{y}[\mathcal{H}(\theta \circ Q)]\right) \circ Q^{-1}=\left(\left(1+\partial_{y} d_{0}\right) \circ Q^{-1}\right)\left\{\partial_{\xi}\left(\mathcal{H} \theta+\mathcal{S}_{(0)} \theta\right)+\delta_{0} \partial_{\tau}\left(\mathcal{H} \theta+\mathcal{S}_{(0)} \theta\right)\right\}$.

Finally the linear equation (6.17) can be re-written as (7.5) with

$$
1+\beta^{(0)}=\left\{q\left(1+\partial_{y} d_{0}\right)\left(1+\partial_{t} e_{0}\right)^{-2}\right\} \circ Q^{-1} .
$$

We now seek $d_{0}(y)$ and $e_{0}(y, t)$ such that $\beta^{(0)}$ is a constant. If this is possible then

$$
\frac{1}{2 \pi} \int_{-\pi}^{\pi}[q(y, t)]^{1 / 2} d t=\left(\frac{1+\beta^{(0)}}{1+\partial_{y} d_{0}(y)}\right)^{1 / 2}
$$

since the $t$-average of $\partial_{t} e_{0}$ is zero. Since the $y$-average of $\partial_{y} d_{0}$ is also zero this yields a formula for $\beta^{(0)}$ in terms of $q$ :

$$
\frac{1}{1+\beta^{(0)}}=4 \pi^{2} \int_{-\pi}^{\pi}\left(\int_{-\pi}^{\pi}[q(y, t)]^{1 / 2} d t\right)^{-2} d y
$$

and with this choice of $\beta^{(0)}$

$$
\begin{aligned}
d_{0}(y) & =\int_{0}^{y}\left\{4 \pi^{2}\left(1+\beta^{(0)}\right)\left(\int_{-\pi}^{\pi}[q(s, t)]^{1 / 2} d t\right)^{-2}-1\right\} d s \in C_{\natural}^{m-3, o} \\
e_{0}(y, t) & =\int_{0}^{t}\left\{\left(\frac{q(y, \tau)\left(1+\partial_{y} d_{0}(y)\right)}{1+\beta^{(0)}}\right)^{1 / 2}-1\right\} d \tau \in C_{\text {如 }}^{m-4, e o},
\end{aligned}
$$


satisfy $1+\beta^{(0)} \equiv\left\{q\left(1+\partial_{y} d_{0}\right)\left(1+\partial_{t} e_{0}\right)^{-2}\right\}$ as required. Since $q$ is even in both variables it is easily checked that $d_{0}( \pm \pi)=0=e_{0}(y, \pm \pi)$ and it follows that $Q$ is a diffeomorphism of the torus with the required properties. The estimate for $\mathcal{G}_{0}(\theta)$ is proved in Appendix L. It essentially follows from Theorem 6.9, Lemma 7.1, the remarks following Definition 6.4, results of Appendix G and Lemma M.2. The last part of the lemma, concerning the range of the linear operator, is a re-statement of the last part of Theorem 6.9 in the new variables.

Remark 7.3. When $\|w\|_{4} \leq M_{4}$ it follows easily, from (5.17), (6.18) and the definition of $p$ in Proposition 6.2, that

$$
\left|\beta^{(0)}-(\mu-1)\right| \leq c\left(M_{4}\right)\|w\|_{4},
$$

and hence, from (7.7) and (7.8), that

$$
\left\|d_{0}\right\|_{C^{l+1}}+\left\|e_{0}\right\|_{C^{l}}+\left\|\dot{e}_{0}\right\|_{C^{l}} \leq c_{l}\left(M_{4}\right)\|w\|_{l+4},
$$

which yields an estimate of the diffeomorphism $Q$. Also

$$
\left\|\frac{\partial_{t t} e_{0}}{\left(1+\partial_{t} e_{0}\right)^{2}}\right\|_{C^{l}} \leq c_{l}\left(M_{4}\right)\|w\|_{l+5}
$$

and $\alpha, \gamma$ and $\delta$ given by Lemma 7.2 satisfy

$$
\|\gamma\|_{C^{l}}+\|\alpha\|_{C^{l}}+\|\delta\|_{C^{l}} \leq c_{l}\left(M_{4}\right)\|w\|_{l+5} .
$$

Remark. In the notation of (6.9),

$$
(\xi, \tau)=\widehat{Q}(x, t)=\left(x+d_{1}(x, t), t+e_{1}(x, t)\right),
$$

where $d_{1} \in C_{\text {弘 }}^{m-3, o e}, e_{1} \in C_{\text {如 }}^{m-4, e o}$ are given by

$$
\begin{aligned}
& d_{1}(x, t)=d(x, t)+d_{0}(x+d(x, t)), \\
& e_{1}(x, t)=e_{0}(x+d(x, t), t) .
\end{aligned}
$$

Therefore, as was foreseen in Remark $6.1, d_{1}$ satisfies (6.10) with different initial conditions:

$$
\begin{aligned}
\partial_{t} d_{1} & =a\left(1+\partial_{x} d_{1}\right), \\
d_{1}(x, 0) & =d_{0}(x) .
\end{aligned}
$$

From (7.12) the Jacobian of the diffeomorphism $(x, t) \mapsto(\xi, \tau)$ is

$$
\begin{aligned}
\left|\frac{\partial(\xi, \tau)}{\partial(x, t)}\right| & =\left(1+\partial_{x} d_{1}\right)\left(1+\partial_{t} e_{1}-a \partial_{x} e_{1}\right) \\
& =\left\{1+\partial_{x} d+\partial_{x} \widehat{d}_{0}\right\}\left(1+\widehat{\partial_{t} e_{0}}\right) .
\end{aligned}
$$




\subsection{The coefficients in (7.5)}

Corollary 7.4. When $w=w_{\varepsilon}^{(N)}$ from Lemma 4.5, with $N \geq 2$, the leading terms of the diffeomorphism $Q$ of the torus are given by

$$
\begin{aligned}
d_{0}(y) & =-2 \varepsilon \sin y+2 \varepsilon^{2} \sin 2 y+O\left(\varepsilon^{3}\right), \\
e_{0}(y, t) & =-\frac{\varepsilon^{2}}{4} \sin 2 t+O\left(\varepsilon^{3}\right),
\end{aligned}
$$

and principal parts of coefficients of (7.5) are given by

$$
\begin{aligned}
\beta^{(0)} & =\frac{\varepsilon^{2}}{4}+O\left(\varepsilon^{3}\right), \quad \delta=O\left(\varepsilon^{3}\right), \\
\gamma & =\varepsilon^{2} \sin 2 \tau+O\left(\varepsilon^{3}\right), \\
\alpha & =2 \varepsilon \sin \xi-2 \varepsilon^{2} \sin 2 \xi+O\left(\varepsilon^{3}\right) . \\
\mathcal{G}_{0}(\theta) & =\varepsilon \mathcal{G}_{01}(\theta)+\varepsilon^{2} \mathcal{G}_{02}(\theta)+O\left(\varepsilon^{3}\|\theta\|\right),
\end{aligned}
$$

with

$$
\mathcal{G}_{01}(\theta)=2(\cos \xi)\left\{(\sin \tau) \pi_{0} \partial_{\tau} \theta+\pi_{0} \theta\right\},
$$

$$
\begin{aligned}
\mathcal{G}_{02}(\theta)=-2(1+\cos 2 \xi)\left\{(\sin \tau) \pi_{0} \partial_{\tau} \theta+\pi_{0} \theta\right\} & \\
& +2 \cos \xi(2 \sin \tau-\sin 2 \tau) \pi_{0}\left(\partial_{\tau} \theta \cos \xi\right) \\
& \quad-\cos \xi(1+\cos 2 \tau) \pi_{0}(\theta \cos \xi) .
\end{aligned}
$$

Proof. From Corollary 6.10 we have

$$
\int_{-\pi}^{\pi}[q(y, t)]^{1 / 2} d t=2 \pi\left\{1+\varepsilon \cos y+\varepsilon^{2}\left(\frac{7}{8}-\frac{5}{4} \cos 2 y\right)+O\left(\varepsilon^{3}\right)\right\},
$$

and so

$$
\left(\int_{-\pi}^{\pi}[q(y, t)]^{1 / 2} d t\right)^{-2}=\frac{1}{4 \pi^{2}}\left\{1-2 \varepsilon \cos y+\varepsilon^{2}\left(-\frac{1}{4}+4 \cos 2 y\right)+O\left(\varepsilon^{3}\right)\right\} .
$$

In (7.6) and (7.7) this gives the required expressions for $\beta^{(0)}$ and $d_{0}(y)$ in powers of $\varepsilon$. It follows that

$$
\frac{q(y, t)\left(1+d_{0}^{\prime}(y)\right)}{1+\beta^{(0)}}=1-\varepsilon^{2} \cos 2 t+O\left(\varepsilon^{3}\right)
$$

which in (7.8) leads to the required expression for $e_{0}$. To calculate $\alpha, \gamma, \delta$ we need to invert $\xi=y+d_{0}(y)$, which gives

$$
y=\xi+2 \varepsilon \sin \xi+O\left(\varepsilon^{3}\right)
$$

from which the expression for $\alpha$ follows. From the formula from $e_{0}$ and Lemma 7.2 we obtain

$$
\gamma=\partial_{t t} e_{0} \circ Q^{-1}+O\left(\varepsilon^{3}\right)=\varepsilon^{2} \sin 2 \tau+O\left(\varepsilon^{3}\right)
$$


Coefficient $\delta$ is $O\left(\varepsilon^{3}\right)$ because $\partial_{y} e_{0}=O\left(\varepsilon^{3}\right)$. To obtain the principal part of $\mathcal{G}_{0}(\theta)$ in terms of $\varepsilon$, note from Lemma 7.1 that $\mathcal{S}_{(0)} \theta=I_{1} \theta+O\left(\varepsilon^{3}\right)$, since $\partial_{y} e_{0}=O\left(\varepsilon^{3}\right)$, where $I_{1}$ is defined in (K.3). Note that in formula (K.3) for $K_{1}$, $\varrho$ denotes the inverse of $y \mapsto \xi+d_{0}(y)$. Let $\varrho(\xi)=\xi+\rho(\xi)$ where, from (7.14), $\left.\rho(\xi)=2 \varepsilon \sin \xi+O\left(\varepsilon^{3}\right)\right)$ as $\varepsilon \rightarrow 0$. Then in the expression (K.3) for $K_{1}$,

$$
\begin{array}{r}
\frac{\sin \frac{1}{2}(\varrho(\xi)-\varrho(\zeta))}{\sin \frac{1}{2}(\xi-\zeta)}=\cos \frac{1}{2}(\rho(\xi)-\rho(\zeta))+\cot \frac{1}{2}(\xi-\zeta) \sin \frac{1}{2}(\rho(\xi)-\rho(\zeta)) \\
=1-\frac{1}{8}(\rho(\xi)-\rho(\zeta))^{2}+\frac{1}{2}(\rho(\xi)-\rho(\zeta)) \cot \frac{1}{2}(\xi-\zeta)+O\left(\varepsilon^{3}\right)
\end{array}
$$

Hence

$$
\begin{aligned}
\log \left|\frac{\sin \frac{1}{2}(\varrho(\xi)-\varrho(\zeta))}{\sin \frac{1}{2}(\xi-\zeta)}\right| & \\
=\frac{1}{2}(\rho(\xi)-\rho(\zeta)) \cot & \frac{1}{2}(\xi-\zeta)-\frac{(\rho(\xi)-\rho(\zeta))^{2}}{\left.8 \sin ^{2} \frac{1}{2}(\xi-\zeta)\right)}+O\left(\varepsilon^{3}\right) \\
= & \varepsilon(\cos \xi+\cos \zeta)-2 \varepsilon^{2} \cos ^{2} \frac{1}{2}(\xi+\zeta)+O\left(\varepsilon^{3}\right) .
\end{aligned}
$$

After an integration by parts this gives

$$
\begin{aligned}
I_{1} \theta & =-\frac{1}{\pi} \int_{-\pi}^{\pi} \partial_{\zeta} \theta\left(\varepsilon(\cos \xi+\cos \zeta)-2 \varepsilon^{2} \cos ^{2} \frac{1}{2}(\xi+\zeta)\right) d \zeta+O\left(\varepsilon^{3}\right) \\
& =-\frac{\varepsilon^{2} \sin \xi}{\pi} \int_{-\pi}^{\pi} \sin \zeta \partial_{\zeta} \theta(\zeta, \tau) d \zeta+O\left(\varepsilon^{3}\right), \quad \text { since } \partial_{\zeta} \theta \text { is odd in } \zeta, \\
& =\frac{\varepsilon^{2} \sin \xi}{\pi} \int_{-\pi}^{\pi} \theta(\zeta, \tau) \cos \zeta d \zeta+O\left(\varepsilon^{3}\right)=2 \varepsilon^{2} \pi_{0}(\theta \cos \xi) \sin \xi+O\left(\varepsilon^{3}\right) .
\end{aligned}
$$

Therefore

$-\left(1+\beta^{(0)}\right)\left\{\partial_{\xi} \mathcal{S}_{(0)} \theta+\delta_{0} \partial_{\tau} \mathcal{S}_{(0)} \theta\right\}+\alpha \mathcal{S}_{(0)} \theta=-2 \varepsilon^{2}(\cos \xi) \pi_{0}(\theta \cos \xi)+O\left(\varepsilon^{3}\|\theta\|\right)$.

To calculate the remaining terms in $\mathcal{G}_{0}(\theta)$ from Corollary 6.10 note that

$$
\begin{aligned}
\left\{\pi_{0}(\theta \circ Q)\right\} \circ Q^{-1} & =\pi_{0} \theta+2 \varepsilon \pi_{0}(\theta \cos \xi)+O\left(\varepsilon^{2}\|\theta\|\right), \\
\left\{\pi_{0}[(\theta \circ Q) \cos y]\right\} \circ Q^{-1} & =\pi_{0}(\theta \cos \xi)+O(\varepsilon\|\theta\|), \\
\left\{\pi_{0}[(\theta \circ Q) \cos 2 y]\right\} \circ Q^{-1} & =\pi_{0}(\theta \cos 2 \xi)+O(\varepsilon\|\theta\|) .
\end{aligned}
$$

This leads to the form of $\mathcal{G}_{0}(\theta)$ in $(7.13)$.

\subsection{Third change of variable - normalization}

We make one further change of variables in (7.5) in an attempt to eliminate the term

$$
(\gamma+\delta \mathcal{H}) \partial_{\tau} \theta+\alpha \mathcal{H} \theta
$$


In fact, we cannot eliminate it entirely, an "average" term remains and in the process we introduce new regularizing and Q1D operators. The idea is to mimic the descent method [22, Section 9] and we need some notation. For any $f \in H_{\text {如 }}^{k}$ the time-average is

$$
\left(\pi_{1} f\right)(\xi)=\frac{1}{2 \pi} \int_{-\pi}^{\pi} f(\xi, \tau) d \tau
$$

and a bounded linear operator $\partial_{\tau}^{-1}$ is defined by

$$
\partial_{\tau}^{-1} f=\left(\mathbb{I}-\pi_{1}\right) \int_{0}^{\tau}\left(\mathbb{I}-\pi_{1}\right) f(\xi, \cdot) d s,
$$

where $\mathbb{I}$ denotes the identity. This is the operator which, in the Fourier expansion of $f$, replaces the functions $\cos p \tau$ and $\sin p \tau$ respectively by $p^{-1} \sin p \tau$ and $-p^{-1} \cos p \tau$ and suppresses the constant terms in $\tau$. For any $f \in H_{\text {啉 }}^{k}$

$$
\partial_{\tau} \partial_{\tau}^{-1} f=\partial_{\tau}^{-1} \partial_{\tau} f=\left(\mathbb{I}-\pi_{1}\right) f \text { and } \partial_{\tau}^{-1} \mathcal{H} f=\mathcal{H} \partial_{\tau}^{-1} f
$$

Suppose that

$$
\begin{aligned}
& \alpha_{0} \in C_{\text {仲 }}^{m-5, e e}, \alpha_{1} \in C_{\text {如 }}^{m-6, e o}, \alpha_{2} \in C_{\text {仲 }}^{m-7, e e}, \\
& \beta_{0} \in C_{\text {仲 }}^{m-5, o e}, \beta_{1} \in C_{\text {吅 }}^{m-6, o o}, \beta_{2} \in C_{\text {仲 }}^{m-7, o e} \text {, }
\end{aligned}
$$

and let

$$
\vartheta=\mathcal{P}^{-1} \theta=\left(1+\left(\alpha_{0}+\beta_{0} \mathcal{H}\right)+\left(\alpha_{1}+\beta_{1} \mathcal{H}\right) \partial_{\tau}^{-1}+\left(\alpha_{2}+\beta_{2} \mathcal{H}\right) \partial_{\tau}^{-2}\right) \theta
$$

where the independent variables $(\xi, \tau)$ are expressed in terms of the original variables by (7.11). Then $\mathcal{P}$ is close to the identity in $H_{\text {如 }}^{k, e e}, k \leq m-7$ and, in the notation of (5.1),

$$
\vartheta=\mathcal{P}^{-1}\left\{\left(\frac{L_{w^{\prime}} \delta w}{1+d^{\prime}}\right) \circ \widehat{Q}^{-1}\right\} .
$$

Remark. From (6.11) and the formulae for $d_{0}$ and $e_{0}$ in Corollary 7.4, it follows that if $w=w_{\varepsilon}^{(N)}, N \geq 2$, from Lemma 4.5,

$$
\begin{aligned}
& d_{1}(x, t)=-2 \varepsilon \cos t \sin x+\varepsilon^{2}(1+\cos 2 t) \sin 2 x+O\left(\varepsilon^{3}\right), \\
& e_{1}(x, t)=-\frac{\varepsilon^{2}}{4} \sin 2 t+O\left(\varepsilon^{3}\right) .
\end{aligned}
$$

Theorem 7.5. (a) For $m \geq 10$, the coefficients in the definition of $\mathcal{P}$ can be chosen so that new function $\vartheta$ satisfies the linear equation

$$
\left.\partial_{\tau \tau} \vartheta-\left(1+\beta^{(0)}\right) \mathcal{H} \partial_{\xi} \vartheta-\kappa^{(0)} \vartheta-\left(\lambda_{0}+\lambda_{1} \mathcal{H}\right) \partial_{\tau}^{-2} \mathcal{P} \vartheta, \vartheta\right)-\mathcal{V}(\vartheta)=h,
$$

where $\beta^{(0)}$ and $\kappa^{(0)}$ are constants, $\lambda_{0} \in C_{\text {耴 }}^{m-8, e e}, \lambda_{1} \in C_{\text {耴 }}^{m-8, o e}$,

$$
h=\mathcal{P}^{-1}\left\{\left(\frac{f\left(1+\dot{e}_{1}-a e_{1}^{\prime}\right)^{-2}}{1+d^{\prime}}\right) \circ \widehat{Q}^{-1}\right\} .
$$

Moreover, $\mathcal{V}=\mathcal{V}_{1}+\mathcal{V}_{2}+\mathcal{V}_{3}$ where 
$\mathcal{V}_{1}$ is a $Q 1 D$ operator of order $(\beta+2, \beta)$ in $H_{\text {吅 }}^{s}$ when $0 \leq s+2 \beta \leq m-8$ for $\beta \leq 3$, and when $0 \leq s+3 \beta \leq m-5$ for $\beta \geq 3$;

$\mathcal{V}_{2}$ is a $Q 1 D$ operator of order $(0, \beta-1)$ in $H_{\text {珀 }}^{s}$ when $0 \leq \beta+s \leq m-8$;

$\mathcal{V}_{3}$ has the property that $\partial_{\tau}^{3} \mathcal{V}_{3}$ is bounded in $H_{\mathrm{qa}}^{s}, 0 \leq s \leq m-10$.

All these operators and coefficients $\lambda_{j}$ have small norms in the following sense: when $w=w_{\varepsilon}^{\left(N_{0}\right)}+\varepsilon^{N_{0}} u, N_{0} \geq 2$,

$$
\begin{aligned}
&\left\|\lambda_{0}\right\|_{C^{s}}+\left\|\lambda_{1}\right\|_{C^{s}} \leq \varepsilon^{2} c_{s}\left(M_{5}\right)\left(1+\varepsilon^{N_{0}-2}\|u\|_{s+8}\right) ; \\
&\left\|G_{\beta+2, \beta} \mathcal{V}_{1}(\vartheta)\right\|_{s} \leq \varepsilon c_{\beta, s}\left(M_{7}\right)\left(\varepsilon^{N_{0}-1}\|u\|_{s+2 \beta+8}\|\vartheta\|_{0}\right. \\
&\left.+\left(1+\varepsilon^{N_{0}-1}\|u\|_{2 \beta+8}\right)\|\vartheta\|_{s}\right) \text { if } \beta \leq 3 ; \\
&\left\|G_{\beta+2, \beta} \mathcal{V}_{1}(\vartheta)\right\|_{s} \leq \varepsilon c_{\beta, s}\left(M_{7}\right)\left(\varepsilon^{N_{0}-1}\|u\|_{s+3 \beta+5}\|\vartheta\|_{0}\right. \\
&\left.+\left(1+\varepsilon^{N_{0}-1}\|u\|_{3 \beta+5}\right)\|\vartheta\|_{s}\right) \text { if } \beta \geq 3 ; \\
&\left\|G_{0, \beta-1} \mathcal{V}_{2}(\vartheta)\right\|_{s} \leq \varepsilon c_{\beta, s}\left(M_{7}\right)\left(\varepsilon^{N_{0}-1}\|u\|_{s+\beta+8}\|\vartheta\|_{0}+\left(1+\varepsilon^{N_{0}-1}\|u\|_{\beta+8}\right)\|\vartheta\|_{s}\right) ; \\
&\left\|\partial_{\tau}^{3} \mathcal{V}_{3}(\vartheta)\right\|_{s} \leq \varepsilon^{2} c_{\beta, s}\left(M_{7}\right)\left(\varepsilon^{N_{0}-2}\|u\|_{s+10}\|\vartheta\|_{0}+\left(1+\varepsilon^{N_{0}-2}\|u\|_{10}\right)\|\vartheta\|_{s}\right) .
\end{aligned}
$$

(b) The range of the linear operator defined by (7.17) is orthogonal in $L_{\text {如 }}^{2}$ to

$$
\mathcal{P}^{*}\left\{\left(\frac{1+\dot{e}_{0}}{1+d_{0}^{\prime}}\right) \circ Q^{-1}\right\}
$$

where $\mathcal{P}^{*}$ denotes the adjoint of $\mathcal{P}$ in $L_{\text {如. }}^{2}$ (c) If $w=w_{\varepsilon}^{\left(N_{0}\right)}, N_{0} \geq 2$, from Lemma 4.5 then, as before, $\beta^{(0)}=\varepsilon^{2} / 4+O\left(\varepsilon^{3}\right)$, and

$$
\begin{aligned}
& \kappa^{(0)}= \varepsilon^{4} / 8+O\left(\varepsilon^{5}\right), \\
& \alpha_{0}(\xi, \tau)= 2 \varepsilon(\cos \xi-1)+\varepsilon^{2}\left(4-\frac{1}{4} \cos 2 \tau-4 \cos \xi\right)+O\left(\varepsilon^{3}\right), \\
& \alpha_{1}(\xi, \tau)=-\frac{\varepsilon^{2}}{4} \sin 2 \tau+O\left(\varepsilon^{3}\right), \\
& \alpha_{2}(\xi, \tau)=-\frac{\varepsilon^{2}}{4}(1-\cos 2 \tau)+O\left(\varepsilon^{3}\right), \\
& \beta_{0}(\xi, \tau)= O\left(\varepsilon^{3}\right), \quad \beta_{1}(\xi, \tau)=O\left(\varepsilon^{3}\right), \quad \beta_{2}(\xi, \tau)=O\left(\varepsilon^{3}\right), \\
& \lambda_{0}(\xi, \tau)=-\varepsilon^{2} \cos 2 \tau+O\left(\varepsilon^{3}\right), \quad \lambda_{1}(\xi, \tau)=O\left(\varepsilon^{3}\right), \\
& \mathcal{P} \vartheta=\vartheta+2 \varepsilon(1-\cos \xi) \vartheta+O\left(\varepsilon^{2}\|\vartheta\|\right), \\
& \mathcal{V}(\vartheta)=-2 \varepsilon(\sin \tau \cos \xi) \pi_{0}\left(\partial_{\tau} \vartheta\right)+2 \varepsilon^{2}(\sin 2 \tau \cos \xi) \pi_{0}\left(\partial_{\tau} \vartheta \cos \xi\right)+ \\
&+\varepsilon^{2}(\cos 2 \tau) \pi_{1} \vartheta+\varepsilon^{2} \cos \xi(1+\cos 2 \tau) \pi_{0}(\vartheta \cos \xi)+O\left(\varepsilon^{3}\|\vartheta\|\right) .
\end{aligned}
$$


The coefficients $\beta^{(0)}, \kappa^{(0)}, \lambda_{0}, \lambda_{1}, \alpha_{j}, \beta_{j}$, and operators $\widehat{Q}$ and $\mathcal{V}$, depend analytically on $(\varepsilon, w)$. (d) If $w=w_{\varepsilon}^{\left(N_{0}\right)}+\varepsilon^{N_{0}} u, N_{0} \geq 3$, and $\pi_{0}\left(\partial_{\tau} \vartheta\right)=0$, then

$$
\mathcal{V}_{j}(\vartheta)=\varepsilon^{2} \mathcal{V}_{j}^{(2)}(\vartheta)+\varepsilon^{3} \mathcal{V}_{j}^{(3)}(\varepsilon)(\vartheta), \quad j=1,2,3,
$$

where, for $m=2,3$,

$$
\begin{aligned}
\left\|G_{\beta+2, \beta} \mathcal{V}_{1}^{(m)}(\vartheta)\right\|_{s} \leq & c_{\beta, s}\left(M_{7}\right)\left(\varepsilon^{N_{0}-m}\|u\|_{s+2 \beta+8}\|\vartheta\|_{0}\right. \\
& \left.+\left(1+\varepsilon^{N_{0}-m}\|u\|_{2 \beta+8}\right)\|\vartheta\|_{s}\right) \text { if } \beta \leq 3 ; \\
\left\|G_{\beta+2, \beta} \mathcal{V}_{1}^{(m)}(\vartheta)\right\|_{s} \leq & c_{\beta, s}\left(M_{7}\right)\left(\varepsilon^{N_{0}-m}\|u\|_{s+3 \beta+5}\|\vartheta\|_{0}\right. \\
& \left.+\left(1+\varepsilon^{N_{0}-m}\|u\|_{3 \beta+5}\right)\|\vartheta\|_{s}\right) \text { if } \beta \geq 3 ; \\
\left\|G_{0, \beta-1} \mathcal{V}_{2}^{(m)}(\vartheta)\right\|_{s} \leq & c_{\beta, s}\left(M_{7}\right)\left(\varepsilon^{N_{0}-m}\|u\|_{s+\beta+8}\|\vartheta\|_{0}\right. \\
& \left.+\left(1+\varepsilon^{N_{0}-m}\|u\|_{\beta+8}\right)\|\vartheta\|_{s}\right) ; \\
\left\|\partial_{\tau}^{3} \mathcal{V}_{3}^{(m)}(\varepsilon)(\vartheta)\right\|_{s} \leq & c_{\beta, s}\left(M_{7}\right)\left(\varepsilon^{N_{0}-m}\|u\|_{s+10}\|\vartheta\|_{0}+\left(1+\varepsilon^{N_{0}-m}\|u\|_{10}\right)\|\vartheta\|_{s}\right) .
\end{aligned}
$$

Proof. The proofs of parts (a), (c) and (d) are in Appendix M. Notice that we have an explicit formula (M.20) for $\kappa^{(0)}$ :

$$
\kappa^{(0)}=\frac{\left(1+\beta^{(0)}\right)^{2}}{16 \pi^{2}} \int_{-\pi}^{\pi} \int_{-\pi}^{\pi} \frac{\left(1+\partial_{t} e_{0}\right)}{\left(1+\partial_{y} d_{0}\right)}\left\{\left(\frac{\partial_{t t} e_{0}}{q}\right)^{2}-\left(\partial_{y} e_{0}\right)^{2}\right\} d y d t .
$$

Now the range of the linear operator defined by (7.17) is orthogonal to

$$
\mathcal{P}^{*}\left\{\left(\frac{1+\partial_{t} e_{0}}{1+\partial_{y} d_{0}}\right) \circ Q^{-1}\right\}
$$

by the result of Lemma 7.2. This ends the proof of the theorem.

Finally in this section we examine the formulae (7.6) for $\beta^{(0)}$ and (7.19) for $\kappa^{(0)}$ when, as in Section 4.3,

$$
(w, \mu)=\left(w_{\varepsilon}^{\left(N_{0}\right)}+\varepsilon^{N_{0}} u, 1+\frac{\varepsilon^{2}}{4}\right) .
$$

Lemma 7.6. Suppose that $N_{0} \geq 4$ and $w$, given by (7.20) has $\|u\|_{5} \leq M_{5}$. Then

$$
\begin{aligned}
& \left|\beta^{(0)}-\frac{\varepsilon^{2}}{4}-\varepsilon^{3} \breve{\beta}(\varepsilon)\right| \leq \varepsilon^{N_{0}} c\left(M_{5}\right)\|u\|_{4} \\
& \left|\kappa^{(0)}-\frac{\varepsilon^{4}}{8}-\varepsilon^{5} \breve{\kappa}(\varepsilon)\right| \leq \varepsilon^{N_{0}+1} c\left(M_{5}\right)\|u\|_{5},
\end{aligned}
$$

where $\breve{\beta}$ and $\breve{\kappa}$ are smooth functions of $\varepsilon$ and $\beta^{(0)}$ and $\kappa^{(0)}$ are smooth functions of $\varepsilon$ and $u$. 
Proof. From (7.9), $\left|\beta^{(0)}-(\mu-1)\right| \leq c(M)\|w\|_{4}$ and, from (7.19) and Remark 7.3 ,

$$
\left|\kappa^{(0)}\right| \leq c(M)\left(\|w\|_{5}\right)^{2} \text { when }\|w\|_{4} \leq M .
$$

The estimate for $\beta^{(0)}$ now follows from (7.9) and the formula for $\beta^{(0)}$ in Corollary 7.4. The estimate for $\kappa^{(0)}$ follows from (M.20), in which the square of a derivative of $e_{0}$ occurs in each term, and the estimate for $e_{0}$ when $u$ in (7.20) is zero from the first part of Corollary 7.4.

\section{Inversion of the Linearized Operator}

We turn to the invertibility of the operator, denoted now by $\mathcal{A}^{(0)}$, in (7.17):

$$
\mathcal{A}^{(0)} \vartheta=\partial_{\tau \tau} \vartheta-\left(1+\beta^{(0)}\right) \mathcal{H} \partial_{\xi} \vartheta-\kappa^{(0)} \vartheta-\left(\lambda_{0}+\lambda_{1} \mathcal{H}\right) \partial_{\tau}^{-2} \mathcal{P} \vartheta-\mathcal{V}(\vartheta) .
$$

\subsection{The kernel and a compatibility condition}

We know from Theorem 7.5 (b) that

$$
\zeta^{(0)}=\mathcal{P}^{*}\left\{\left(\frac{1+\dot{e_{0}}}{1+d_{0}^{\prime}}\right) \circ Q^{-1}\right\}
$$

which is close to 1 , is orthogonal to the range of $\mathcal{A}^{(0)}$. Let $\mathbb{P}$ denote the projection defined on $L_{\text {如 }}^{2}$ by

$$
\mathbb{P} f=f-\pi_{0} \pi_{1} f \text { where } \pi_{0} \pi_{1} f=\frac{1}{4 \pi^{2}} \int_{-\pi}^{\pi} \int_{-\pi}^{\pi} f(\xi, \tau) d \xi d \tau .
$$

Let $H_{\perp}^{k}=\mathbb{P} H_{\text {叫 }}^{k}, k \in \mathbb{N}$.

Lemma 8.1. Suppose that $\mathbb{P} \mathcal{A}^{(0)} \mathbb{P}: H_{\perp}^{k} \rightarrow H_{\perp}^{k^{\prime}}$ is a bijection for some $k, k^{\prime} \in$ $\mathbb{N}$. Then $\operatorname{ker} \mathcal{A}^{(0)}=\operatorname{span}\left\{\vartheta^{(0)}\right\}$ for some $\vartheta^{(0)}$ in $H_{\text {㕷 }}^{\text {,ee }}$ for any $m$, and the range of $\mathcal{A}^{(0)}$ is $\left\{u \in H_{\text {始 }}^{k^{\prime}}:\left\langle u, \zeta^{(0)}\right\rangle_{L^{2}}=0\right\}$.

Proof. Consider the equation $\mathcal{A}^{(0)} \vartheta=0$ where $\vartheta=(I-\mathbb{P}) \vartheta+\mathbb{P} \vartheta=\alpha 1+\varpi$, say. Then $\varpi=-\alpha\left(\mathbb{P} \mathcal{A}^{(0)} \mathbb{P}\right)^{-1} \mathbb{P} \mathcal{A}^{(0)} 1$ and $\vartheta^{(0)}=1-\left(\mathbb{P} \mathcal{A}^{(0)} \mathbb{P}\right)^{-1} \mathbb{P} \mathcal{A}^{(0)} 1 \in H_{\natural \natural}^{m, e e}$ is in the kernel of $\mathbb{P} \mathcal{A}^{(0)}$. Hence $\mathcal{A}^{(0)} \vartheta^{(0)}=\beta 1$ for some $\beta$. Since 1 and $\zeta^{(0)}$ are not orthogonal and the range of $\mathcal{A}^{(0)}$ is orthogonal to $\zeta^{(0)}$ it follows that $\beta=0$ and so $\operatorname{ker} \mathcal{A}^{(0)}=\operatorname{span}\left\{\vartheta^{(0)}\right\}$. Now let $h$ be orthogonal to $\zeta^{(0)}$ and seek a solution of the equation $\mathcal{A}^{(0)} \vartheta=h$ where $\left\langle\vartheta, \vartheta^{(0)}\right\rangle_{L^{2}}=0$. Then $\vartheta=\varpi+\alpha 1$ where $\varpi=\mathbb{P} \vartheta$ and $\mathbb{P} \mathcal{A}^{(0)}(\varpi+\alpha 1)=\mathbb{P} h$. This implies that

$$
\varpi=\left(\mathbb{P} \mathcal{A}^{(0)} \mathbb{P}\right)^{-1} \mathbb{P}\left(-\alpha \mathcal{A}^{(0)} 1+\mathbb{P} h\right)=-\alpha+\alpha \vartheta^{(0)}+\left(\mathbb{P} \mathcal{A}^{(0)} \mathbb{P}\right)^{-1} \mathbb{P} h .
$$

Since $\varpi+\alpha$ is orthogonal to $\vartheta^{(0)}$ this gives a formula for $\alpha$ in terms of $h$. With this choice of $\alpha$ and $\varpi$,

$$
\mathbb{P} \mathcal{A}^{(0)}(\varpi+\alpha 1)=\mathbb{P} \mathcal{A}^{(0)}\left(\mathbb{P} \mathcal{A}^{(0)} \mathbb{P}\right)^{-1} \mathbb{P} h=\mathbb{P} h,
$$


which shows that $\mathcal{A}^{(0)}(\varpi+\alpha 1)-h=\beta 1$. Since both $h$ and the range of $\mathcal{A}^{(0)}$ are orthogonal to $\zeta^{(0)}$, which is not orthogonal to 1 , it follows that $\beta=0$. This proves the result.

Thus it is sufficient to prove that the linear operator is invertible in the subspace orthogonal to constants.

\subsection{Principal part of $\mathbb{P} \mathcal{A}^{(0)} \mathbb{P}$ when $\boldsymbol{w}=\boldsymbol{w}_{\varepsilon}^{(N)}, \quad \boldsymbol{N} \geq \mathbf{2}$}

In this section we consider what happens when $w=w_{\varepsilon}^{(N)}, N \geq 2$, from Lemma 4.5 and compute $\mathbb{P} \mathcal{A}^{(0)} \mathbb{P}$ explicitly up to order $\varepsilon^{2}$. From the proof of Lemma 8.1

$$
\vartheta^{(0)}=1-\left(\mathbb{P} \mathcal{A}^{(0)} \mathbb{P}\right)^{-1} \mathbb{P} \mathcal{A}^{(0)} 1,
$$

where

$$
\begin{aligned}
\mathbb{P}^{(0)} 1 & =-\mathbb{P}\left(\lambda_{0}+\lambda_{1} \mathcal{H}\right) \partial_{\tau}^{-2} \mathcal{P} 1-\mathbb{P} \mathcal{V}(1), \\
\mathbb{P}\left(\lambda_{0}+\lambda_{1} \mathcal{H}\right) \partial_{\tau}^{-2} \mathcal{P} 1 & =O\left(\varepsilon^{3}\right) \text { since } \partial_{\tau}^{-2} \mathcal{P} 1=O(\varepsilon), \\
\mathbb{P} \mathcal{V}(1) & =\varepsilon^{2} \cos 2 \tau+O\left(\varepsilon^{3}\right) .
\end{aligned}
$$

Hence

$$
\mathbb{P} \mathcal{A}^{(0)} 1=-\varepsilon^{2} \cos 2 \tau+O\left(\varepsilon^{3}\right), \quad P_{0} \mathcal{A}^{(0)} 1=O\left(\varepsilon^{3}\right), \quad\left(I-P_{0}\right) \mathbb{P} \mathcal{A}^{(0)} 1=O\left(\varepsilon^{2}\right),
$$

where $P_{0}$ is defined by (8.3) in the next section. In the light of $(8.4 \mathrm{a})$ and $(8.4 \mathrm{~b})$, it follows that (for a precise estimate see Lemma 8.6)

$$
\vartheta^{(0)}=1-\left(\mathbb{P} \mathcal{A}^{(0)} \mathbb{P}\right)^{-1} \mathbb{P} \mathcal{A}^{(0)} 1=1+O(\varepsilon) .
$$

From Corollary 7.4

$$
\left(\frac{1+\dot{e_{0}}}{1+d_{0}^{\prime}}\right)=1+2 \varepsilon \cos y+\varepsilon^{2}\left(2-\frac{1}{2} \cos 2 t-2 \cos 2 y\right)+O\left(\varepsilon^{3}\right)
$$

whence

$$
\left(\frac{1+\dot{e_{0}}}{1+d_{0}^{\prime}}\right) \circ Q^{-1}=1+2 \varepsilon \cos \xi-\frac{\varepsilon^{2}}{2} \cos 2 \tau+O\left(\varepsilon^{3}\right)
$$

and, since

$$
\mathcal{P}^{*}=1-\alpha_{0}+\alpha_{0}^{2}+\partial_{\tau}^{-1} \alpha_{1}-\partial_{\tau}^{-2} \alpha_{2}+O\left(\varepsilon^{3}\right),
$$

we find that

$$
\zeta^{(0)}=1+2 \varepsilon+O\left(\varepsilon^{2}\right) .
$$

is orthogonal to the range of $\mathcal{A}^{(0)}$. In the Fourier-series notation (4.6), $\vartheta \in$ $\mathbb{P} H_{\text {ตt }}^{k, e e}$ has the form

$$
\vartheta=\sum_{n+q \geq 1} \vartheta_{n}^{(q)} \cos n \xi \cos q \tau
$$


and the $(n, q)$-Fourier components of $\partial_{\tau \tau} \vartheta-\left(1+\beta^{(0)}\right) \mathcal{H} \partial_{\xi} \vartheta-\kappa^{(0)} \mathbb{P} \vartheta$ is

$$
\left\{\left(1+\beta^{(0)}\right) n-q^{2}-\kappa^{(0)}\right\} \vartheta_{n}^{(q)}, \quad n+q \geq 1,
$$

where $\beta^{(0)}=\varepsilon^{2} / 4+O\left(\varepsilon^{3}\right), \kappa^{(0)}=\varepsilon^{4} / 8+O\left(\varepsilon^{5}\right)$. Note that this operator is diagonal and an expected small divisor problem appears when we try to invert it. Now we consider the $(n, q)$-Fourier components of $-\mathbb{P}\left(\lambda_{0}+\lambda_{1} \mathcal{H}\right) \partial_{\tau}^{-2} \mathcal{P} \vartheta$. From Theorem 7.5 (c), $\mathcal{P} \vartheta=\vartheta+O(\varepsilon\|\vartheta\|), \lambda_{0}(\xi, \tau)=-\varepsilon^{2} \cos 2 \tau+O\left(\varepsilon^{3}\right)$ and $\lambda_{1}(\xi, \tau)=O\left(\varepsilon^{3}\right)$. Therefore, up to order $\varepsilon^{2}$,

$$
\left(-\mathbb{P}\left(\lambda_{0}+\lambda_{1} \mathcal{H}\right) \partial_{\tau}^{-2} \mathcal{P} \vartheta\right)_{n}^{(q)}= \begin{cases}-\frac{\varepsilon^{2}}{2}\left\{\frac{1}{(q-2)^{2}} \vartheta_{n}^{(q-2)}+\frac{1}{(q+2)^{2}} \vartheta_{n}^{(q+2)}\right\} & q \geq 3, \\ -\frac{\varepsilon^{2}}{32} \vartheta_{n}^{(4)} & q=2, \\ -\frac{\varepsilon^{2}}{2}\left\{\frac{1}{9} \vartheta_{n}^{(3)}+\vartheta_{n}^{(1)}\right\} & q=1, \\ -\frac{\varepsilon^{2}}{8} \vartheta_{n}^{(2)} & q=0 .\end{cases}
$$

It remains to find the $(n, q)$-Fourier components of $-\mathbb{P} \mathcal{V}(\vartheta)$. Consider first the term of order $\varepsilon$ :

$$
2 \varepsilon \mathbb{P}\left\{(\sin \tau \cos \xi) \pi_{0}\left(\partial_{\tau} \vartheta\right)\right\} .
$$

In this expression the projection $\mathbb{P}$ plays no rôle and

$$
\begin{aligned}
\left(2 \varepsilon(\sin \tau \cos \xi) \pi_{0}\left(\partial_{\tau} \vartheta\right)\right)_{n}^{(q)} & n \neq 1, \\
& =\left\{\begin{array}{lr}
0 & \\
\varepsilon\left\{(q-1) \vartheta_{0}^{(q-1)}-(q+1) \vartheta_{0}^{(q+1)}\right\} & n=1, q \geq 2, \\
-2 \varepsilon \vartheta_{0}^{(2)} & (n, q)=(1,1), \\
-\varepsilon u_{0}^{(1)} & (n, q)=(1,0) .
\end{array}\right.
\end{aligned}
$$

The terms of order $\varepsilon^{2}$ in $-\mathbb{P V}(\vartheta)$ are

$$
\begin{aligned}
& -2 \varepsilon^{2} \mathbb{P}\left\{(\sin 2 \tau \cos \xi) \pi_{0}\left(\partial_{\tau} \vartheta \cos \xi\right)\right\}-\varepsilon^{2} \mathbb{P}\left\{(\cos 2 \tau) \pi_{1} \vartheta\right\}+ \\
& -\varepsilon^{2} \mathbb{P}\left\{\cos \xi(1+\cos 2 \tau) \pi_{0}(\vartheta \cos \xi)\right\}+O\left(\varepsilon^{3}\|\vartheta\|\right)
\end{aligned}
$$

and

$$
-(\mathbb{P} \mathcal{V}(\vartheta))_{n}^{(q)}= \begin{cases}0 & \text { for } n \neq 1, q \neq 2, \\ -\varepsilon^{2} \vartheta_{n}^{(0)} & \text { for } n \neq 1, q=2 .\end{cases}
$$

For $n=1$ and $q \geq 3$,

$$
-(\mathbb{P} \mathcal{V}(\vartheta))_{1}^{(q)}=\varepsilon^{2}\left\{\frac{-1}{2} \vartheta_{1}^{(q)}+\left(\frac{3}{4}-\frac{q}{2}\right) \vartheta_{1}^{(q-2)}+\left(\frac{3}{4}+\frac{q}{2}\right) \vartheta_{1}^{(q+2)}\right\}
$$

and, for $n=1$ and $q \leq 2$,

$$
-(\mathbb{P} \mathcal{V}(\vartheta))_{1}^{(q)}= \begin{cases}\varepsilon^{2}\left\{-2 \vartheta_{1}^{(0)}+\frac{7}{4} \vartheta_{1}^{(4)}\right\} & \text { for } q=2, \\ \varepsilon^{2}\left\{-\frac{1}{4} \vartheta_{1}^{(1)}+\frac{5}{4} \vartheta_{1}^{(3)}\right\} & \text { for } q=1, \\ \varepsilon^{2}\left\{-\frac{1}{2} \vartheta_{1}^{(0)}+\frac{3}{4} \vartheta_{1}^{(2)}\right\} & \text { for } q=0 .\end{cases}
$$




\subsection{Structure of the linear operator}

Here we consider the component of the main part of $\mathbb{P} \mathcal{A}^{(0)} \vartheta$ which lies in the kernel of $\mathcal{L}_{1}:=\partial_{\tau \tau}-\mathcal{H} \partial_{\xi}$. In other words, we examine the Fourier-series coefficients with $(n, q)=\left(q^{2}, q\right)$. In this case $(8.2)$ becomes $\left(q^{2} \beta^{(0)}-\kappa^{(0)}\right) \vartheta_{q^{2}}^{(q)}$ and so, when $w=w_{\varepsilon}^{(N)}$, this part of the operator is diagonal with, by Lemma 7.6, coefficients

$$
q^{2}\left(\frac{\varepsilon^{2}}{4}+O\left(\varepsilon^{3}\right)\right) \text { as } \varepsilon \rightarrow 0
$$

Now observe that when $\left(q^{2}, q\right)=(1,1)$ there is a term $-2 \varepsilon \vartheta_{0}^{(2)}$ in $-\mathbb{P} \mathcal{V}(\vartheta)$, and all the other terms in $\mathbb{P} \mathcal{A}^{(0)}(\vartheta)$ have coefficients which are $O\left(\varepsilon^{2}\right)$ as $\varepsilon \rightarrow 0$. This suggests that we should write $\vartheta=\Theta+\varepsilon \Psi$, where

$$
\Theta=P_{0} \vartheta=\sum_{q \geq 1} u_{q^{2}}^{(q)} \cos q^{2} \xi \cos q \tau \text { and } \Psi=\sum_{n \neq q^{2}} y_{n}^{(q)} \cos n \xi \cos q \tau \text {. }
$$

With this decomposition, the linear equation $\mathcal{A}^{(0)} \vartheta=h$ is equivalent to

$$
\begin{aligned}
\mathcal{M}_{\varepsilon} \Theta+\mathcal{A}_{\varepsilon}^{(0)} \Psi & =\varepsilon^{-2} P_{0} h, \\
\left(\Lambda_{\varepsilon}^{(0)}+\varepsilon \Lambda_{\varepsilon}^{(1)}\right) \Psi+\varepsilon \mathcal{K}_{\varepsilon} \Theta & =\varepsilon^{-1}\left(\mathbb{I}-P_{0}\right) \mathbb{P} h
\end{aligned}
$$

where

$$
\begin{aligned}
P_{0} \mathcal{A}^{(0)} P_{0} & =\varepsilon^{2} \mathcal{M}_{\varepsilon}, \\
P_{0} \mathcal{A}^{(0)} \mathbb{P}\left(\mathbb{I}-P_{0}\right) & =\varepsilon \mathcal{A}_{\varepsilon}^{(0)}, \\
\left(\mathbb{I}-P_{0}\right) \mathbb{P} \mathcal{A}^{(0)} \mathbb{P}\left(\mathbb{I}-P_{0}\right) & =\Lambda_{\varepsilon}^{(0)}+\varepsilon \Lambda_{\varepsilon}^{(1)}, \\
\left(\mathbb{I}-P_{0}\right) \mathbb{P} \mathcal{A}^{(0)} P_{0} & =\varepsilon^{2} \mathcal{K}_{\varepsilon},
\end{aligned}
$$

with

$$
\begin{aligned}
& \Lambda_{\varepsilon}^{(0)}=\partial_{\tau \tau}-\left(1+\beta^{(0)}\right) \mathcal{H} \partial_{\xi}-\kappa^{(0)}, \\
& \left(\Lambda_{\varepsilon}^{(0)} \Psi\right)_{n}^{(q)}=\left\{\left(1+\beta^{(0)}\right) n-q^{2}-\kappa^{(0)}\right\} y_{n}^{(q)} \text {, } \\
& \left\{\mathcal{M}_{0} \Theta\right\}_{q^{2}}^{(q)}=\left\{\begin{array}{cc}
\frac{q^{2}}{4} \vartheta_{q^{2}}^{(q)} & \text { for } q \neq 1, \\
-\frac{1}{2} \vartheta_{1}^{(1)} & \text { for } q=1
\end{array}\right. \\
& \left\{\mathcal{A}_{0}^{(0)} \Psi\right\}_{q^{2}}^{(q)}= \begin{cases}0 & \text { for } q \neq 1, \\
-2 y_{0}^{(2)} & \text { for } q=1,\end{cases} \\
& \left\{\mathcal{K}_{0} \Theta\right\}_{n}^{(q)}=\left\{\begin{array}{lr}
\frac{-1}{2(q-2)^{2}} \vartheta_{(q-2)^{2}}^{(q-2)} & \text { for } n=(q-2)^{2}, q \geq 3 \\
\frac{-1}{2(q+2)^{2}} \vartheta_{(q+2)^{2}}^{(q+2)} & \text { for } n=(q+2)^{2}, q \geq 0 \\
0 & \text { otherwise }
\end{array}\right. \\
& \left(\Lambda_{0}^{(1)} \Psi\right)_{n}^{(q)}=\left\{\begin{array}{lr}
(q-1) y_{0}^{(q-1)}-(q+1) y_{0}^{(q+1)} & \text { for } n=1, q \geq 2, \\
-y_{0}^{(1)} & \text { for }(n, q)=(1,0) \\
0 \text { otherwise. } &
\end{array}\right.
\end{aligned}
$$




\subsection{Inversion of $\mathcal{A}^{(0)}$}

We begin with two lemmas that estimate the operators in (8.4).

Lemma 8.2. There exists $\varepsilon_{0}>0$ such that, for $\|u\|_{10} \leq M_{10}$ and $\varepsilon \leq \varepsilon_{0}$

$$
\begin{aligned}
\left\|\mathcal{M}_{\varepsilon}^{-1} \Theta\right\|_{s} & \leq c_{s}\left(M_{10}\right)\left(\|u\|_{s+8}\|\Theta\|_{0}+\|\Theta\|_{s-1}\right), \\
\left\|\mathcal{M}_{\varepsilon}^{-1} \mathcal{A}_{\varepsilon}^{(0)} \Psi\right\|_{s} & \leq c_{s}\left(M_{10}\right)\left(\|u\|_{s+8}\|\Psi\|_{0}+\|\Psi\|_{s}\right)
\end{aligned}
$$

which leads to precise estimates of the solution $\Theta$ of equation (8.4a):

$$
\Theta=-\mathcal{M}_{\varepsilon}^{-1} \mathcal{A}_{\varepsilon}^{(0)} \Psi+\varepsilon^{-2} \mathcal{M}_{\varepsilon}^{-1} P_{0} h .
$$

Proof. The formula for $\mathcal{M}_{0}(\Theta)$ (which coincides with the coefficient of $\varepsilon^{2}$ in the formula for $\mathbb{P} \mathcal{A}^{(0)} \mathbb{P}$ in Section 8.2) implies that, for any $s \geq 0$,

$$
\left\|\mathcal{M}_{0}^{-1} \Theta\right\|_{s+1} \leq c_{s}\|\Theta\|_{s}
$$

Since $\Theta=P_{0} \Theta$, the hypotheses of Theorem 7.5 (d) hold, and hence, for $\varepsilon>0$,

$$
\begin{aligned}
\mathcal{M}_{\varepsilon}^{(1)}(\Theta)= & \varepsilon^{-1}\left(\mathcal{M}_{\varepsilon}-\mathcal{M}_{0}\right) \Theta=\varepsilon^{-3}\left(P_{0} \mathcal{A}^{(0)}(\Theta)-\varepsilon^{2} \mathcal{M}_{0}(\Theta)\right) \\
= & \frac{1}{\varepsilon^{3}}\left(-\beta^{(0)} \mathcal{H} \Theta^{\prime}-\kappa^{(0)} \Theta\right. \\
& \left.\quad-P_{0}\left(\left(\lambda_{0}+\lambda_{1} \mathcal{H}\right) \partial_{\tau}^{-2} \mathcal{P} \Theta+\mathcal{V}(\Theta)\right)-\varepsilon^{2} \mathcal{M}_{0}(\Theta)\right) \\
= & \frac{1}{\varepsilon^{3}}\left(-\left(\beta^{(0)}-\frac{\varepsilon^{2}}{4}\right) \mathcal{H} \Theta^{\prime}-\kappa^{(0)} \Theta\right. \\
& -P_{0}\left(\left(\lambda_{0}+\varepsilon^{2} \cos 2 \tau+\lambda_{1} \mathcal{H}\right) \partial_{\tau}^{-2} \mathcal{P} \Theta\right)-P_{0}\left(\varepsilon^{2} \mathcal{V}^{(2)}+\varepsilon^{3} \mathcal{V}^{(3)}(\varepsilon)\right)(\Theta) \\
& \left.\quad-\frac{\varepsilon^{2}}{4} \mathcal{H} \Theta^{\prime}+\varepsilon^{2} P_{0}\left(\cos 2 \tau \partial_{\tau}^{-2} \mathcal{P}(\Theta)\right)-\varepsilon^{2} \mathcal{M}_{0}(\Theta)\right) \\
= & \frac{1}{\varepsilon^{3}}\left(-\left(\beta^{(0)}-\frac{\varepsilon^{2}}{4}\right) \mathcal{H} \Theta^{\prime}-\kappa^{(0)} \Theta\right. \\
& -P_{0}\left(\left(\lambda_{0}+\varepsilon^{2} \cos 2 \tau+\lambda_{1} \mathcal{H}\right) \partial_{\tau}^{-2} \mathcal{P} \Theta\right)-\varepsilon^{3} P_{0} \mathcal{V}^{(3)}(\varepsilon)(\Theta) \\
& \left.\quad+\varepsilon^{2} P_{0}\left(\cos 2 \tau \partial_{\tau}^{-2}((\mathcal{P}-\mathbb{I}) \Theta)\right)\right) .
\end{aligned}
$$

From the estimates for $\lambda_{0}, \lambda_{1}, \beta^{(0)}$ and $\kappa^{(0)}$ in Theorem 7.5 and Lemma 7.6, and from the estimate (M.19) of the change of variable $\mathcal{P}$, we obtain

$$
\begin{array}{r}
\left\|\left(\beta^{(0)}-\frac{\varepsilon^{2}}{4}\right) \mathcal{H} \Theta^{\prime}+\kappa^{(0)} \Theta+P_{0}\left(\lambda_{0}+\varepsilon^{2} \cos 2 \tau+\lambda_{1} \mathcal{H}\right) \partial_{\tau}^{-2} \mathcal{P} \Theta\right\|_{s-1} \\
\leq \varepsilon^{3} c\left(M_{8}\right)\left(\|u\|_{s+8}\|\Theta\|_{0}+\|\Theta\|_{s}\right) .
\end{array}
$$


Moreover, from Theorem 7.5 (d) with $N_{0} \geq 3$,

$$
\begin{aligned}
\left\|P_{0} \mathcal{V}_{1}^{(3)}(\Theta)\right\|_{s-1} & \leq c\left\|G_{2,0} P_{0} \mathcal{V}_{1}^{(3)}(\Theta)\right\|_{s} \\
& \leq c\left(M_{8}\right)\left(\|u\|_{s+8}\|\Theta\|_{0}+\|\Theta\|_{s}\right), \\
\left\|P_{0} \mathcal{V}_{2}^{(3)}(\Theta)\right\|_{s-1} & \leq c\left(M_{8}\right)\left(\|u\|_{s+8}\|\Theta\|_{0}+\left(1+\|u\|_{9}\right)\|\Theta\|_{s}\right), \\
\left\|P_{0} \mathcal{V}_{3}^{(3)}(\Theta)\right\|_{s-1} & \leq c\left\|P_{0} \partial_{\tau}^{3} \mathcal{V}_{3}^{(3)}(\Theta)\right\|_{s-2} \\
& \leq c\left(M_{8}\right)\left(\|u\|_{s+8}\|\Theta\|_{0}+\left(1+\|u\|_{10}\right)\|\Theta\|_{s}\right) .
\end{aligned}
$$

Combining these estimates, we find that

$$
\left\|\mathcal{M}_{\varepsilon}^{(1)} \Theta\right\|_{s-1} \leq c_{s}\left(M_{10}\right)\left\{\|u\|_{s+8}\|\Theta\|_{0}+\|\Theta\|_{s}\right\},
$$

which with (8.8) leads to the conclusion that

$$
\left\|\mathcal{M}_{0}^{-1} \mathcal{M}_{\varepsilon}^{(1)} \Theta\right\|_{s} \leq c_{s}\left(M_{10}\right)\left\{\|u\|_{s+8}\|\Theta\|_{0}+\|\Theta\|_{s}\right\} .
$$

A combination of (8.10) with the special case of (8.10) when $s=0$ and the formula

$$
\mathcal{M}_{\varepsilon}^{-1}=\left(1+\varepsilon \mathcal{M}_{0}^{-1} \mathcal{M}_{\varepsilon}^{(1)}\right)^{-1} \mathcal{M}_{0}^{-1},
$$

leads to the estimate (8.6). We proceed in the same way for $\mathcal{A}_{\varepsilon}^{(0)} \Psi$ as for $\mathcal{M}_{\varepsilon}^{(1)} \Theta$ except that $\mathcal{V}(\Psi)=O(\varepsilon)$ while $\mathcal{V}(\Theta)=O\left(\varepsilon^{2}\right)$, which explains the respective orders in $\varepsilon$ of each term. The estimate for $\left\|\mathcal{M}_{\varepsilon}^{-1} \mathcal{A}_{\varepsilon}^{(0)} \Psi\right\|_{s}$ is then the same as for $\left\|\mathcal{M}_{0}^{-1} \mathcal{M}_{\varepsilon}^{(1)} \Theta\right\|_{s}$.

Lemma 8.3. Suppose that $\|u\|_{17} \leq M_{17}$ and that $\beta^{(0)}$ and $\kappa^{(0)}$ satisfy the Diophantine condition that, for some $c>0$,

$$
\mid\left(q^{2}-\left(1+\beta^{(0)}\right) p-\kappa^{(0)} \mid \geq c / q^{2} \text { for all }(p, q) \text { with } p \neq q^{2} \neq 0 .\right.
$$

Then

$$
\left\|\left(\Lambda_{\varepsilon}^{(0)}\right)^{-1}\left\{\Lambda_{\varepsilon}^{(1)}-\mathcal{K}_{\varepsilon} \mathcal{M}_{\varepsilon}^{-1} \mathcal{A}_{\varepsilon}^{(0)}\right\} \Psi\right\|_{s} \leq c_{s}\left(M_{17}\right)\left(\|u\|_{s+17}\|\Psi\|_{0}+\|\Psi\|_{s}\right) .
$$

Proof. To estimate $\left\|\left(\Lambda_{\varepsilon}^{(0)}\right)^{-1} \mathcal{K}_{\varepsilon} \mathcal{M}_{\varepsilon}^{-1} \mathcal{A}_{\varepsilon}^{(0)} \Psi\right\|_{s}$, let $\underline{\Theta}$ be an arbitrary function with $\underline{\Theta}=P_{0} \underline{\Theta}$. From (8.1) and the formula following (8.4) we have

$$
\left.\varepsilon^{2} \mathcal{K}_{\varepsilon} \underline{\Theta}=-\left(1-P_{0}\right) \mathbb{P}\left\{\lambda_{0}+\lambda_{1} \mathcal{H}\right) \partial_{\tau}^{-2} \mathcal{P} \underline{\Theta}+\mathcal{V}(\underline{\Theta})\right\}
$$

where, by Theorem 7.5 (d),

$$
\begin{gathered}
\left\|G_{6,4}\left(1-P_{0}\right) \mathbb{P} \mathcal{V}_{1}(\underline{\Theta})\right\|_{s} \leq \varepsilon^{2} c_{s}\left(M_{17}\right)\left\{\|u\|_{s+17}\|\underline{\Theta}\|_{0}+\|\underline{\Theta}\|_{s}\right\}, \\
\left\|G_{0,1}\left(1-P_{0}\right) \mathbb{P} \mathcal{V}_{2}(\underline{\Theta})\right\|_{s} \leq \varepsilon^{2} c_{s}\left(M_{10}\right)\left\{\|u\|_{s+10}\|\underline{\Theta}\|_{0}+\|\underline{\Theta}\|_{s}\right\}, \\
\left.\| \partial_{\tau}^{2}\left(1-P_{0}\right) \mathbb{P}\left\{\lambda_{0}+\lambda_{1} \mathcal{H}\right) \partial_{\tau}^{-2} \mathcal{P} \underline{\Theta}+\mathcal{V}_{3}(\underline{\Theta})\right\} \|_{s} \\
\leq \varepsilon^{2} c_{s}\left(M_{10}\right)\left\{\|u\|_{s+10}\|\underline{\Theta}\|_{0}+\|\underline{\Theta}\|_{s}\right\} .
\end{gathered}
$$


From the definition of $\Lambda_{\varepsilon}^{(0)}$ and the Diophantine condition (8.11) it is immediate that, in the subspace $\left(\mathbb{I}-P_{0}\right) H_{\perp}^{s, e e}$,

$$
\left\|\left(\Lambda_{\varepsilon}^{(0)}\right)^{-1} f\right\|_{s} \leq c\left\|\partial_{\tau}^{2} f\right\|_{s}
$$

which, with the estimates on $\lambda_{0}, \lambda_{1}$ and $\mathcal{V}_{3}$ from Theorem 7.5 (a), can be used to establish the required bound on the term

$$
\left.\left(\Lambda_{\varepsilon}^{(0)}\right)^{-1}\left(\left(1-P_{0}\right) \mathbb{P}\left\{\lambda_{0}+\lambda_{1} \mathcal{H}\right) \partial_{\tau}^{-2} \mathcal{P} \underline{\Theta}+\mathcal{V}_{3}(\underline{\Theta})\right\}\right) .
$$

To estimate $\left(\Lambda_{\varepsilon}^{(0)}\right)^{-1} f$, where $f=\left(1-P_{0}\right) \mathbb{P} \mathcal{V}_{1}(\underline{\Theta})$ or $\left(1-P_{0}\right) \mathbb{P} \mathcal{V}_{2}(\underline{\Theta})$, we write

$$
\left(\Lambda_{\varepsilon}^{(0)}\right)^{-1}=\left(\Lambda_{\varepsilon}^{(0)}\right)^{-1} \Pi_{1}+\left(\Lambda_{\varepsilon}^{(0)}\right)^{-1}\left(\mathbb{I}-\Pi_{1}\right)
$$

where $\Pi_{1}$ is the orthogonal projection onto the space of functions $f$ with Fourier coefficients $f_{p}^{(q)}$ such that $q^{2}>2 p$. For $q^{2}>2 p$

$$
\mid\left(q^{2}-\left(1+\beta^{(0)}\right) p-\kappa^{(0)} \mid \geq c^{\prime} \max \left\{q^{2}, p\right\}\right.
$$

and, for $q^{2} \leq 2 p$, because of (8.11),

$$
\mid\left(q^{2}-\left(1+\beta^{(0)}\right) p-\kappa^{(0)} \mid \geq \frac{c}{q^{2}} \frac{q^{2}}{2 p}=\frac{c}{2 p} \geq \frac{c}{2 p}\left(\frac{q^{2}}{2 p}\right)^{3}=c^{\prime} \frac{q^{6}}{p^{4}} .\right.
$$

Therefore

$$
\begin{gathered}
\left\|\left(\Lambda_{\varepsilon}^{(0)}\right)^{-1} \Pi_{1} f\right\|_{s} \leq c \min \left\{\left\|\partial_{\tau}^{-2} \Pi_{1} f\right\|_{s},\left\|\partial_{\xi}^{-1} \Pi_{1} f\right\|_{s}\right\} \\
\left\|\left(\Lambda_{\varepsilon}^{(0)}\right)^{-1}\left(\mathbb{I}-\Pi_{1}\right) f\right\|_{s} \leq c \min \left\{\left\|\partial_{\tau}^{-6} \partial_{\xi}^{4}\left(\mathbb{I}-\Pi_{1}\right) f\right\|_{s},\left\|\partial_{\xi}\left(\mathbb{I}-\Pi_{1}\right) f\right\|_{s}\right\} .
\end{gathered}
$$

If now $\underline{\Theta}=\mathcal{M}_{\varepsilon}^{-1} \mathcal{A}_{\varepsilon}^{(0)} \Psi$ where (8.7) holds,

$$
\left\|\left(\Lambda_{\varepsilon}^{(0)}\right)^{-1}\left\{\mathcal{K}_{\varepsilon} \mathcal{M}_{\varepsilon}^{-1} \mathcal{A}_{\varepsilon}^{(0)}\right\} \Psi\right\|_{s} \leq c_{s}\left(M_{17}\right)\left(\|u\|_{s+17}\|\Psi\|_{0}+\|\Psi\|_{s}\right) .
$$

In the same way (except for an extra order of $\varepsilon$ in $\mathcal{V}(\mathbb{P} \Psi)$ ), we obtain a similar estimate for $\left\|\left(\Lambda_{\varepsilon}^{(0)}\right)^{-1} \Lambda_{\varepsilon}^{(1)} \Psi\right\|_{s}$. This proves the lemma.

Theorem 8.4. Suppose that $\|u\|_{17} \leq M_{17}$ and that $\beta^{(0)}$ and $\kappa^{(0)}$ satisfy the Diophantine condition (8.11). Let $h \in H_{\text {如 }}^{\text {s,ee }}, s \geq 2$, satisfy the compatibility condition $\left\langle h, \zeta^{(0)}\right\rangle=0$. Then any solution of the equation $\mathcal{A}^{(0)} \vartheta=h$ is given by $\vartheta=\vartheta_{1}+\alpha \vartheta^{(0)}$ where $(\mathbb{I}-\mathbb{P}) \vartheta_{1}=0$, and for $s \geq 2$ and $\varepsilon \leq \varepsilon_{0}$ sufficiently small,

$$
\left\|\vartheta_{1}\right\|_{s-2} \leq \frac{c_{s}\left(M_{17}\right)}{\varepsilon^{2}}\left(\|u\|_{s+15}\|h\|_{0}+\|h\|_{s}\right) .
$$

Proof. Since, by (8.4),

$$
\left(\Lambda_{\varepsilon}^{(0)}+\varepsilon \Lambda_{\varepsilon}^{(1)}\right) \Psi-\varepsilon \mathcal{K}_{\varepsilon} \mathcal{M}_{\varepsilon}^{-1} \mathcal{A}_{\varepsilon}^{(0)} \Psi=\varepsilon^{-1}\left\{\left(1-P_{0}\right) \mathbb{P} h-\mathcal{K}_{\varepsilon} \mathcal{M}_{\varepsilon}^{-1} P_{0} h\right\},
$$


and (8.11) holds, Lemma 8.3 gives an estimate for $\Psi$,

$\|\Psi\|_{s-2} \leq \varepsilon c_{s}\left(M_{17}\right)\left(\|u\|_{s+15}\|\Psi\|_{0}+\|\Psi\|_{s-2}\right)+\frac{c_{s}\left(M_{10}\right)}{\varepsilon}\left(\|u\|_{s+10}\|h\|_{0}+\|h\|_{s}\right)$.

Now the case $s=2$ gives a bound for $\|\Psi\|_{0}$ which, when substituted into the general formula gives, for $\varepsilon$ sufficiently small,

$$
\|\Psi\|_{s-2} \leq \frac{c_{s}\left(M_{17}\right)}{\varepsilon}\left(\|u\|_{s+15}\|h\|_{0}+\|h\|_{s}\right) .
$$

Combined with the estimates in Lemma 8.2 for $\Theta=-\mathcal{M}_{\varepsilon}^{-1} \mathcal{A}_{\varepsilon}^{(0)} \Psi+\varepsilon^{-2} \mathcal{M}_{\varepsilon}^{-1} P_{0} h$, in the light of Lemma 8.1, we obtain the result of the theorem.

\subsection{Inversion of $\Lambda(u, \varepsilon)$}

Now we show that the approximate linearized operator $\Lambda(u, \varepsilon)$ defined by $(6.3)$ satisfies (O.12) in Appendix O. This leads to the conclusion that hypotheses (D) and (E) in the Nash-Moser theory of Appendix N are satisfied in the standingwave problem.

Theorem 8.5. There exists $\varepsilon_{0}>0$ such that if $\varepsilon \in\left(0, \varepsilon_{0}\right),\|u\|_{17} \leq M_{17}$ and $\beta^{(0)}$ and $\kappa^{(0)}$ satisfy the Diophantine condition (8.11), then, for $f$ with

$$
\underline{f} \in H_{\text {如 }}^{s, e e}, s \geq 2,\langle\underline{f}, 1\rangle=0,
$$

the system

$$
\Lambda(u, \varepsilon) \underline{u}=\underline{f}, \quad\langle\underline{u}, 1\rangle=0
$$

has a unique solution $\underline{u}$ and

$$
\|\underline{u}\|_{s-2} \leq \frac{c_{s}\left(M_{17}\right)}{\varepsilon^{2}}\left\{\|u\|_{s+15}\|\underline{f}\|_{0}+\|\underline{f}\|_{s}\right\} .
$$

Proof. By (6.3), (6.7), Theorem 6.9 and Lemma 7.2, $\Lambda L_{w^{\prime}}^{-1} v=f$ implies that $\theta=(\tilde{v} p) \circ Q^{-1}$ satisfies (7.5). Then Theorem 7.5 says that $\vartheta=\mathcal{P}^{-1} \theta$ satisfies

$$
\left(1+d^{\prime}\right)\left(1+\dot{e}_{1}-a e_{1}^{\prime}\right)^{2}\left(\mathcal{P} \mathcal{A}^{(0)} \vartheta\right) \circ \widehat{Q}=f=\Lambda L_{w^{\prime}}^{-1} v=\Lambda L_{w^{\prime}}^{-1}\left\{\left(\frac{(\mathcal{P} \vartheta) \circ Q}{p}\right)^{\wedge}\right\} .
$$

This gives

$\left\{p_{1}^{-1} \mathcal{P} \mathcal{A}^{(0)} \vartheta\right\} \circ \widehat{Q}=\Lambda(u, \varepsilon) \underline{u}, \quad$ equivalently $\mathcal{A}^{(0)} \vartheta=\mathcal{P}^{-1}\left\{p_{1}(\Lambda(u, \varepsilon) \underline{u}) \circ \widehat{Q}^{-1}\right\}$,

where

$$
\underline{u}=L_{w^{\prime}}^{-1}\left\{\left(1+d^{\prime}\right)(\mathcal{P} \vartheta \circ \widehat{Q})\right\}, \quad p_{1}=\left(\frac{p}{\left(1+\dot{e}_{0}\right)^{2}}\right) \circ Q^{-1} .
$$

From (7.10), (7.11), (G.3) and Lemma G.1, we find that $(\xi, \tau)=\widehat{Q}(x, t)$, and $(\widehat{Q}-\mathbb{I})(x, t)=\left(d_{1}(x, t), e_{1}(x, t)\right)$ where

$$
\left\|d_{1}\right\|_{C^{s}}+\left\|e_{1}\right\|_{C^{s}} \leq c_{s}\left(M_{4}\right)\|w\|_{s+4} .
$$


Now from (M.19) and Lemma G.3,

$$
\|\mathcal{P} \vartheta \circ \widehat{Q}\|_{s} \leq c_{s}\left(M_{7}\right)\left(\|\vartheta\|_{s}+\|w\|_{s+7}\|\vartheta\|_{0}\right),
$$

and hence, from (G.3),

$$
\|\underline{u}\|_{s} \leq c_{s}\left(M_{7}\right)\left(\|\vartheta\|_{s}+\|u\|_{s+7}\|\vartheta\|_{0}\right) .
$$

Lemma 8.6. For $\|u\|_{17} \leq M_{17}$, the function $\vartheta^{(0)}$ which spans the kernel of $\mathcal{A}^{(0)}$ satisfies

$$
\begin{aligned}
\vartheta^{(0)} & =1-\left(\mathbb{P} \mathcal{A}^{(0)} \mathbb{P}\right)^{-1} \mathbb{P} \mathcal{A}^{(0)} 1 \\
\left\|\vartheta^{(0)}-1\right\|_{s} & \leq \varepsilon c_{s}\left(M_{17}\right)\left(1+\|u\|_{s+17}\right) .
\end{aligned}
$$

Proof. The expression for $\vartheta^{(0)}$ is given in Lemma 8.1. Now, from (M.21), (M.19), the fact that $\partial_{\tau}^{-1} 1=0$, and Theorem 7.5 (c) we obtain

$$
\left\|\mathbb{P}\left(\lambda_{0}+\lambda_{1} \mathcal{H}\right) \partial_{\tau}^{-2} \mathcal{P} 1\right\|_{s} \leq \varepsilon^{3} c_{s}\left(M_{7}\right)\left(1+\|u\|_{s+8}\right) .
$$

In the notation of Theorem 7.5 (d), $\mathcal{V}^{(2)}(1)=\cos 2 \tau$, by (7.18). It follows, as in the proof of (8.9), that

$$
\left\|\mathbb{P} \mathcal{V}(1)-\varepsilon^{2} \cos 2 \tau\right\|_{s} \leq \varepsilon^{3} c_{s}\left(M_{7}\right)\left(1+\|u\|_{s+10}\right),
$$

and therefore

$$
\begin{aligned}
\left\|P_{0} \mathcal{A}^{(0)} 1\right\|_{s} & \leq \varepsilon^{3} c_{s}\left(M_{7}\right)\left(1+\|u\|_{s+10}\right), \\
\left\|\left(\mathbb{I}-P_{0}\right) \mathbb{P} \mathcal{A}^{(0)} 1\right\|_{s} & \leq \varepsilon^{2} c_{s}\left(M_{7}\right)\left(1+\|u\|_{s+10}\right) .
\end{aligned}
$$

We then need to use a refined version of Theorem 8.4 which distinguishes the components $P_{0} h$ and $\left(\mathbb{I}-P_{0}\right) \mathbb{P} h$. In the present case $P_{0} h=O\left(\varepsilon^{3}\right)$, while $(\mathbb{I}-$ $\left.P_{0}\right) \mathbb{P} h=O\left(\varepsilon^{2}\right)$, which gives that the right-hand sides of equations (8.4a) and (8.4b) are of order $O(\varepsilon)$. The result of the lemma then follows from the proof of Theorem 8.4.

We now show that the zero-mean condition on $\underline{u}$ in (8.12) leads to an estimate of $\alpha$ in $\vartheta=\vartheta_{1}+\alpha \vartheta^{(0)}$. Indeed, from (8.13),

$$
\left\|L_{w^{\prime}}^{-1}\left\{\left(1+d^{\prime}\right)(\mathcal{P} 1 \circ \widehat{Q})\right\}-1\right\|_{s} \leq \varepsilon c_{s}\left(M_{7}\right)\left(1+\|u\|_{s+7}\right),
$$

and hence, from Lemma 8.6,

$$
\left\|L_{w^{\prime}}^{-1}\left\{\left(1+d^{\prime}\right)\left(\mathcal{P} \vartheta^{(0)} \circ \widehat{Q}\right)\right\}-1\right\|_{s} \leq \varepsilon c_{s}\left(M_{17}\right)\left(1+\|u\|_{s+17}\right) .
$$

Since $\underline{u}$ has zero mean on $[-\pi, \pi] \times[-\pi, \pi]$,

$$
\left\langle L_{w^{\prime}}^{-1}\left\{\left(1+d^{\prime}\right)\left(\mathcal{P} \vartheta_{1} \circ \widehat{Q}\right)\right\}+\alpha L_{w^{\prime}}^{-1}\left\{\left(1+d^{\prime}\right)\left(\mathcal{P} \vartheta^{(0)} \circ \widehat{Q}\right)\right\}, 1\right\rangle=0,
$$


and since $\vartheta_{1}$ is uniquely defined and $\left\langle\vartheta_{1}, 1\right\rangle=0$,

$$
|\alpha|=\left|\frac{\left\langle L_{w^{\prime}}^{-1}\left\{\left(1+d^{\prime}\right)\left(\mathcal{P} \vartheta_{1} \circ \widehat{Q}\right)\right\}, 1\right\rangle}{\left\langle L_{w^{\prime}}^{-1}\left\{\left(1+d^{\prime}\right)\left(\mathcal{P} \vartheta^{(0)} \circ \widehat{Q}\right)\right\}, 1\right\rangle}\right| \leq c_{s}\left(M_{17}\right)|| \vartheta_{1} \|_{0} .
$$

Finally,

$$
\|\underline{u}\|_{s-2} \leq c_{s}\left(M_{17}\right)\left\{\|u\|_{s+15}\left\|\vartheta_{1}\right\|_{0}+\left\|\vartheta_{1}\right\|_{s-2}\right\}
$$

where

$$
\left\|\vartheta_{1}\right\|_{s-2} \leq \frac{c_{s}\left(M_{17}\right)}{\varepsilon^{2}}\left(\|u\|_{s+15}\|h\|_{0}+\|h\|_{s}\right)
$$

and hence

$$
\|\underline{u}\|_{s-2} \leq \frac{c_{s}\left(M_{17}\right)}{\varepsilon^{2}}\left\{\|u\|_{s+15}\|h\|_{0}+\|h\|_{s}\right\}
$$

Now we want to solve

$$
\Lambda(u, \varepsilon) \underline{u}=\underline{f}, \quad\langle\underline{u}, 1\rangle=0
$$

when the given function $\underline{f}$ is orthogonal to constants. If such a solution exists,

$$
\underline{f}=\left\{p_{1}^{-1} \mathcal{P} h\right\} \circ \widehat{Q}, \quad h=\mathcal{P}^{-1}\left\{p_{1} \underline{f} \circ \widehat{Q}^{-1}\right\},
$$

and, from (6.20), (7.10), (G.3) and Lemma G.1,

$$
\left\|p_{1}\right\|_{C^{s}} \leq c_{s}\left(M_{4}\right)\left(1+\|w\|_{s+4}\right) .
$$

Finally, using Lemma G.3 and (M.19) this gives

$$
\|h\|_{s} \leq c_{s}\left(M_{7}\right)\left\{\|u\|_{s+7}\|\underline{f}\|_{0}+\|\underline{f}\|_{s}\right\}
$$

and (8.14) leads to the result of Theorem 8.5.

\section{Existence of Standing Waves}

In this section we use the Nash-Moser theorem from Appendix $\mathrm{N}$ to prove that for every $\varepsilon>0$ in a subset $\mathcal{E}$ of the interval $\left(0, \varepsilon_{0}\right)$, where the set $\mathcal{E}$ is dense at 0 $\left(\lim _{r \searrow 0} r^{-1} \operatorname{meas}(\mathcal{E} \cap[0, r])=1\right)$, there exists a non-constant solution $w \in H_{\text {吼 }}^{17, e e}$ of equation (4.1). Since functions in $H_{\text {比 }}^{17 \text {,ee }}$ have 15 continuous derivatives and are doubly-periodic in $x$ and $t$, this gives a classical solution of the standing-wave problem on infinite depth (see the last sentence of Section 2).

Theorem 9.1. There exists a measurable set $\mathcal{E} \subset\left[0, \varepsilon_{0}\right]$ which is dense at 0 such that, for any $\varepsilon \in \mathcal{E}$, there exists a solution $w \in H_{\text {如 }}^{17, e e}$ of (4.1) with $\mu=1+\varepsilon^{2} / 4$ and $\pi_{0} \pi_{1}(w)=0$. The function $\varepsilon \mapsto w$ is Lipschitz continuous and $w=w_{\varepsilon}^{\left(N_{0}\right)}+o\left(\varepsilon^{N_{0}}\right)$ for $N_{0} \geq 4$, where $w_{\varepsilon}^{(N)}=\varepsilon \cos x \cos t+O\left(\varepsilon^{2}\right)$ is given in Lemma 4.5. 
Remark. In [14] it is shown that there are many alternatives for the approximate solution $w_{\varepsilon}^{\left(N_{0}\right)}$, and for most of our proof it does not matter which one is chosen. However at certain points we make use of the particular choice given by Lemma 4.5 in order

(i) to compute explicitly the coefficients $\beta^{(0)}$ and $\kappa^{(0)}$;

(ii) to find an explicit expression for the approximate linearized operator $\Lambda$ to order $\varepsilon^{2}$;

(iii) to show the surprising fact that the linear operator $\mathcal{M}_{0}$ in the infinite dimensional bifurcation equation has a regularizing inverse (see (8.6)). It is natural to ask whether the method applies for other choices of approximate solutions. It does; in particular $\beta^{(0)}$ and $\kappa^{(0)}$ have the same orders in $\varepsilon$, but there is a complication due to the fact that, for all other choices of approximate solutions, the operator $\mathcal{V}$ is such that $\left(\mathbb{I}-P_{0}\right) \mathcal{V} P_{0}$ is $O(\varepsilon)$ instead of being $O\left(\varepsilon^{2}\right)$ as it is here. For the sake of conciseness, only the family $w_{\varepsilon}^{(N)}$ given by Lemma 4.5 will be treated here. The alternatives for $w_{\varepsilon}^{(N)}$ given in [14] will be dealt with elsewhere.

Proof. We use the formulation (4.10) and so we need to show that $\Phi$ defined in (4.9) satisfies the hypotheses in Appendix N. To verify (N.1a) and (N.1e), we use estimates (4.11) and (6.6) for $\mathcal{F}(w, \mu)$ and $\Gamma\left(\mathcal{F}(w, \mu), L_{w^{\prime}}(\cdot)\right)$ with

$$
w=w_{\varepsilon}^{\left(N_{0}\right)}+\varepsilon^{N_{0}} u, \quad \mu=1+\varepsilon^{2} / 4 .
$$

and for (N.1d) recall the definition of $\Lambda$ in (6.3). Then conditions (N.1a) and (N.1d) are satisfied by choosing $E_{r}:=H_{\perp}^{r, e e}=H_{\text {吼 }}^{r, e e} \cap\{1\}^{\perp}, F_{\rho}=H_{\perp}^{\rho, e e}$ and

$$
1 \leq \rho \leq r-2 .
$$

Because of the term involving $\|w\|_{l+4}$ in (6.6), condition (N.1e) is satisfied when

$$
1 \leq \rho \leq r-3 .
$$

From Theorem 8.5 we see that condition (N.1h) is satisfied when

$$
2 \leq \rho \leq r-15, \quad \sigma=2, \quad \varrho=2 .
$$

Note from Lemma 3.1 that $\mathcal{F}(\cdot, \mu)$ is defined and real-analytic in a ball $B$ of sufficiently small radius about the origin in $H_{\perp}^{r, e e}, r \geq 3$ (we need that $w \in B$ implies that $\left.\left|\mathcal{H} w^{\prime}\right|<1\right)$. To verify hypotheses (N.1b) and (N.1c) on

$$
D(u, v, \varepsilon)=\Phi(u, \varepsilon)-\Phi(v, \varepsilon)-\Phi_{u}^{\prime}(v, \varepsilon)(u-v)
$$

we use the analyticity of the mapping $\Phi: B \times \mathbb{R} \rightarrow H_{\perp}^{\rho, e e}$, and the formulae

$$
D(u, v, \varepsilon)=\int_{0}^{1} \int_{0}^{\tau} \Phi_{u u}^{\prime \prime}(v+\eta(u-v), \varepsilon)(u-v, u-v) d \eta d \tau,
$$




$$
\begin{array}{r}
\begin{array}{r}
D\left(u_{1}, v_{1}, \varepsilon_{1}\right)-D\left(u_{2}, v_{2}, \varepsilon_{2}\right) \\
=\int_{0}^{1}\left\{D_{u}^{\prime}\left(u_{s}, v_{s}, \varepsilon_{s}\right)\left(u_{1}-u_{2}\right)+D_{v}^{\prime}\left(u_{s}, v_{s}, \varepsilon_{s}\right)\left(v_{1}-v_{2}\right)\right. \\
\left.+D_{\varepsilon}^{\prime}\left(u_{s}, v_{s}, \varepsilon_{s}\right)\left(\varepsilon_{1}-\varepsilon_{2}\right)\right\} d s, \\
D_{u}^{\prime}\left(u_{s}, v_{s}, \varepsilon_{s}\right)\left(u_{1}-u_{2}\right) \\
=\int_{0}^{1} \int_{0}^{\tau} \Phi_{u u u}^{\prime \prime \prime}\left(v_{s}+\eta\left(u_{s}-v_{s}\right), \varepsilon\right)\left(u_{s}-v_{s}, u_{s}-v_{s}, u_{1}-u_{2}\right) d \eta d \tau \\
\quad+\int_{0}^{1} \int_{0}^{\tau} 2 \Phi_{u u}^{\prime \prime \prime}\left(v_{s}+\eta\left(u_{s}-v_{s}\right), \varepsilon\right)\left(u_{s}-v_{s}, u_{1}-u_{2}\right) d \eta d \tau,
\end{array}
\end{array}
$$

where

$$
u_{s}=u_{2}+s\left(u_{1}-u_{2}\right), \quad v_{s}=v_{2}+s\left(v_{1}-v_{2}\right), \quad \varepsilon_{s}=\varepsilon_{2}+s\left(\varepsilon_{1}-\varepsilon_{2}\right),
$$

It is clear from Sections 3 and 4 that we need estimates of products of $(u-v)$ and $\Phi_{j}$, which may involve the constant operator $\mathcal{H}$, where

$$
\left\|\Phi_{j}\right\|_{\rho+l} \leq c_{l}\left(M_{r}\right)\left(1+\|u\|_{r+l}\right)
$$

for $1 \leq \rho \leq r-2$ (in particular for $r=3, \rho=1$ ). By (5.18) and standard interpolation this gives the required estimates when $\rho=2, r=17$ (in fact $r=4$ is sufficient). Notice that for (N.1c) that we have used the fact that

$$
\left\|u_{s}-v_{s}\right\|_{r+l} \leq\left\|u_{1}-v_{1}\right\|_{r+l}+\left\|u_{2}-v_{2}\right\|_{r+l} .
$$

Estimates (N.1f) and (N.1g) follow by the same method now using the realanalyticity of $\Phi$ and $\Lambda$, the formulae

$$
\Phi\left(u_{1}, \varepsilon_{1}\right)-\Phi\left(u_{2}, \varepsilon_{2}\right)=\int_{0}^{1}\left\{\Phi_{u}^{\prime}\left(u_{s}, \varepsilon_{s}\right)\left(u_{2}-u_{1}\right)+\Phi_{\varepsilon}^{\prime}\left(u_{s}, \varepsilon_{s}\right)\left(\varepsilon_{2}-\varepsilon_{1}\right)\right\} d s
$$

and

$$
\begin{aligned}
\left(\Phi_{u}^{\prime}\left(u_{1}, \varepsilon_{1}\right)-\Phi_{u}^{\prime}\right. & \left.\left(u_{2}, \varepsilon_{2}\right)\right) v \\
& =\int_{0}^{1}\left\{\Phi_{u u}^{\prime \prime}\left(u_{s}, \varepsilon_{s}\right)\left(u_{2}-u_{1}, v\right)+\left(\varepsilon_{2}-\varepsilon_{1}\right) \Phi_{u \varepsilon}^{\prime \prime}\left(u_{s}, \varepsilon_{s}\right) v\right\} d s,
\end{aligned}
$$

and an analogous formula for $\Lambda_{u}^{\prime}$ in place of $\Phi_{u}^{\prime}$. Lemma 7.6 implies that we need $N_{0} \geq 4$ if (O.5) is to hold. Then Corollary O.3 holds for the set $\mathcal{E}$ and, since $(\mathrm{O} .12)$ is satisfied by Theorem 8.5 , this means that hypothesis (D) holds. Moreover Theorem O.8 (which uses (O.5)) implies that (E) is satisfied. In other words, the subsets $\mathcal{E}\left(\nu_{j}\right)$ are dense at 0 with an intersection that is also dense at 0 . We can then apply Theorem N.2, since Lemma 4.5 provides an approximate smooth solution $u_{\varepsilon}^{(N)}$ that satisfies, trivially, (N.3) and (N.4) for any integer $N$. The theorem is then proved. 
Remark 9.2. It is worth pointing out a consequence of the scale invariance of the standing-wave problem on infinite depth and the fact that all positive rationals are eigenvalues of the problem linearized at 0 (see Section 1.1). Up to now we have been looking for solutions of $(\mathrm{K})$ and $(\mathrm{D})$ in Section 2 in which $1+\varepsilon^{2} / 4=\mu=g T^{2} / 2 \pi \lambda$ and $w$, the wave elevation, is of the form $\varepsilon \cos x \cos t+$ $O\left(\varepsilon^{2}\right)$. (As in (1.1), $T$ and $\lambda$ are the spatial and temporal periods.) Suppose instead we seek solutions with smaller minimal periods $2 \pi / p_{0}$ and $2 \pi / q_{0}$ and with asymptotic form

$$
\varepsilon \cos p_{0} x \cos q_{0} t+O\left(\varepsilon^{2}\right), \quad p_{0}, q_{0} \in \mathbb{N} .
$$

Then a change of scales $\tilde{\lambda}=\lambda / p_{0}$ and $\tilde{T}=T / q_{0}$ changes $\mu$ into $\tilde{\mu}=\mu p_{0} / q_{0}^{2}$, and Theorem 9.1 leads to the bifurcation of standing waves with $\tilde{\mu} \backslash 1$. Since every positive rational number $r$ can be written as $q_{0}^{2} / p_{0}$, the scale invariance and Theorem 9.1 leads to the observation that standing waves which are 'unimodal' in this sense bifurcate from $\mu=r$, for every $r \in \mathbb{Q}^{+}$. 


\section{A Proof of Lemma 3.1}

We first observe that (3.4) comes from the fact that $\mathcal{H}^{*}=-\mathcal{H}$ in $\mathcal{L}\left(L_{\natural}^{2}\right)$, and (3.3) is immediate. The identity (3.5) comes from the fact that $\pi_{0}\left(w^{\prime}\right)=0$ and from (3.2). The identity (3.6) is obtained by taking the adjoint of (3.5) using (3.3). Now we show that $L_{w^{\prime}}$ has a bounded inverse given by (3.7). The equation $L_{w^{\prime}} u=f$, where $f$ is given in $L_{\sharp}^{2}$, can be written as

$$
\operatorname{Re}\left(\frac{u+i \mathcal{H} u}{1+\mathcal{H} w^{\prime}-i w^{\prime}}\right)=\frac{f}{D} \text { on } \mathbb{R} \text {. }
$$

Since $\left|\mathcal{H} w^{\prime}\right|<1$ on $\mathbb{R}$, the function $1+\mathcal{H} w^{\prime}-i w^{\prime}$ can be extended, using the Poisson integral formula, as a bounded analytic function on the lower half-plane. This extension is $2 \pi$-periodic in $x$, its real part is nowhere zero, and it converges uniformly, as $y \rightarrow-\infty$, to 1 . Therefore the function

$$
\frac{u+i \mathcal{H} u}{1+\mathcal{H} w^{\prime}-i w^{\prime}}
$$

may be extended as an analytic function in the lower complex half-plane which is $2 \pi$-periodic in $x$ and its average on an interval of length $2 \pi$ parallel to $x$ axis (independent of $y<0)$ is $\pi_{0}(u)$, which is real. Therefore

$$
\frac{u+i \mathcal{H} u}{1+\mathcal{H} w^{\prime}-i w^{\prime}}=(1+i \mathcal{H}) \frac{f}{D},
$$

and

$$
u=\operatorname{Re}\left\{\left(1+\mathcal{H} w^{\prime}-i w^{\prime}\right)(1+i \mathcal{H}) \frac{f}{D}\right\}=\widetilde{L}_{w^{\prime}}\left(\frac{f}{D}\right) .
$$

It follows that if $\widetilde{L}_{w^{\prime}} v=g$, then $v=\frac{1}{D} L_{w^{\prime}} g$. The formulae (3.9) for the inverses of $M_{w^{\prime}}$ and $\widetilde{M}_{w^{\prime}}$ are established by considering the adjoints of $L_{w^{\prime}}^{-1}$ and $\widetilde{L}_{w^{\prime}}^{-1}$ since, by (3.3),

$$
\left(L_{w^{\prime}}^{-1}\right)^{*}=M_{w^{\prime}}^{-1} \text { and } \quad\left(\widetilde{L}_{w^{\prime}}^{-1}\right)^{*}=\widetilde{M}_{w^{\prime}}^{-1} .
$$

Now, from(3.5) and (3.7),

$$
\mathcal{H}\left(\frac{1}{D} L_{w^{\prime}}\right)-\left(\frac{1}{D} L_{w^{\prime}}\right) \mathcal{H}=\left(\frac{w^{\prime}}{D}\right) \pi_{0}\left(\frac{1}{D} L_{w^{\prime}}\right),
$$

where we have used the fact that $L_{w^{\prime}} w^{\prime} \equiv w^{\prime}$. To prove (3.8), we use the remarkable identity

$$
\pi_{0}\left(\frac{1}{D} L_{w^{\prime}}\right)=\pi_{0}
$$

which comes from the fact that $\widetilde{M}_{w^{\prime}}(1)=1$. Hence, by $(3.9), M_{w^{\prime}}(1 / D)=1$ (this is not obvious directly) and, for any $f \in L_{\natural}^{2}$,

$$
\begin{aligned}
\pi_{0}\left(\frac{1}{D} L_{w^{\prime}} f\right) & =\frac{1}{2 \pi}\left\langle L_{w^{\prime}} f, 1 / D\right\rangle=\frac{1}{2 \pi}\left\langle f, M_{w^{\prime}}(1 / D)\right\rangle \\
& =\frac{1}{2 \pi}\langle f, 1\rangle=\pi_{0}(f) .
\end{aligned}
$$


The formula for the commutator of $M_{w^{\prime}}$ and $\mathcal{H}$ is obtained by taking the adjoint of (3.8). It remains to establish (3.10) which, because of (3.8) and (3.9), is equivalent to

$$
\widetilde{M}_{w^{\prime}} J(f, g)=\mathcal{H}\left(f L_{w^{\prime}} g\right)+f L_{w^{\prime}} \mathcal{H} g+\left(w^{\prime} f\right) \pi_{0}(g) .
$$

That this identity holds follows by expanding both sides, using (3.2) with $f$ and $g$ replaced by $f g$ and $w^{\prime}$, and noting that $\pi_{0}\left(w^{\prime}\right)=0$. This ends the proof of the lemma.

\section{B Calculation of Approximate Solutions}

To find an explicit formula for the approximate solution in Section 4.2 we need to solve the system for $w^{(1)}$ and $w^{(2)}$ :

$$
\begin{aligned}
0= & \mathcal{L}_{1} w^{(2)}+\mathcal{N}_{2}\left(w^{(1)}, w^{(1)}\right) \\
0= & \mathcal{L}_{1} w^{(3)}-\frac{1}{4} \mathcal{H} w^{\prime(1)}+2 \mathcal{N}_{2}\left(w^{(1)}, w^{(2)}\right)+\mathcal{N}_{3}\left(w^{(1)}, w^{(1)}, w^{(1)}\right), \\
0= & \mathcal{L}_{1} w^{(4)}-\frac{1}{4} \mathcal{H} w^{\prime(2)}+2 \mathcal{N}_{2}\left(w^{(1)}, w^{(3)}\right)+\mathcal{N}_{2}\left(w^{(2)}, w^{(2)}\right) \\
& +3 \mathcal{N}_{3}\left(w^{(1)}, w^{(1)}, w^{(2)}\right)+\mathcal{N}_{4}\left(w^{(1)}, w^{(1)}, w^{(1)}, w^{(1)}\right),
\end{aligned}
$$

where

$$
\begin{aligned}
\mathcal{N}_{4}(w, w, w, w)=2 \mathcal{H} \partial_{x}\left\{3\left(\mathcal{H}\left(w^{\prime} \mathcal{H} \dot{w}\right)\right)^{2}-w^{\prime 2}(\mathcal{H} \dot{w})^{2}-2(\mathcal{H} \dot{w})\left(\mathcal{H} w^{\prime}\right) \mathcal{H}\left(w^{\prime} \mathcal{H} \dot{w}\right)\right\} \\
+\partial_{x}\left\{\mathcal{H}\left(w^{\prime 2}\right)-3 w^{\prime} \mathcal{H} w^{\prime}\right\}+2 w^{\prime \prime}\left(\pi_{0} \dot{w}\right) \pi_{0}\left[\dot{w}, w^{\prime}\right] .
\end{aligned}
$$

The first equation leads to

$$
\begin{aligned}
0 & =\ddot{w}^{(2)}-\mathcal{H} w^{(2) \prime}+\partial_{t}\left(\frac{1}{2} \sin 2 t\right)+\frac{1}{2} \mathcal{H} \partial_{x}\left((1-2 \cos 2 x) \sin ^{2} t\right) \\
& =\ddot{w}^{(2)}-\mathcal{H} w^{(2) \prime}+\cos 2 t+\cos 2 x(1-\cos 2 t)
\end{aligned}
$$

hence we obtain $w^{(2)}$ (with zero average, and involving only even multiples of $x$ and $t$ )

$$
w^{(2)}=\frac{1}{4} \cos 2 t-\frac{1}{2} \cos 2 x(1+\cos 2 t) .
$$

To calculate $w^{(3)}$ we need

$$
\begin{aligned}
2 \mathcal{N}_{2}\left(w^{(1)}, w^{(2)}\right)= & \partial_{t}\left[-\sin t(1+\cos 2 t)-\frac{1}{2} \sin 2 t \cos t\right] \cos x+ \\
& \partial_{x}\left[\sin t \sin 2 t \sin 3 x+\sin t \sin 2 t\left(\frac{3}{2} \sin x-3 \sin 3 x\right)\right] \\
= & -3 \cos x \cos 3 t-3 \cos 3 x(\cos t-\cos 3 t),
\end{aligned}
$$


and

$$
\begin{aligned}
\mathcal{N}_{3}\left(w^{(1)}, w^{(1)}, w^{(1)}\right) & =-\frac{1}{2} \sin t \sin 2 t(\cos x-3 \cos 3 x) \\
& =-\frac{1}{4}(\cos t-\cos 3 t)(\cos x-3 \cos 3 x)
\end{aligned}
$$

The equation for $w^{(3)}$ now gives

$$
0=\ddot{w}^{(3)}-\mathcal{H} w^{(3) \prime}-\frac{11}{4} \cos x \cos 3 t-\frac{9}{4} \cos 3 x(\cos t-\cos 3 t) .
$$

The compatibility condition is therefore satisfied (there are no term in $\cos x \cos t$ ) and this justifies, a posteriori, the choice $\mu=1+\varepsilon^{2} / 4$. Hence we obtain

$$
w^{(3)}=A^{(3)} \cos x \cos t-\frac{11}{32} \cos x \cos 3 t+\frac{3}{8} \cos 3 x(3 \cos t+\cos 3 t)
$$

where $A^{(3)}$ is still unknown, and is determined by a calculation at order $\varepsilon^{5}$. To calculate $w^{(4)}$, we obtain a system of the form

$0=\ddot{w}^{(4)}-\mathcal{H} w^{(4) \prime}+2 A^{(3)}\{\cos 2 t+\cos 2 x(1-\cos 2 t)\}+\sum_{0 \leq p, q \leq 2} a_{p q} \cos 2 p x \cos 2 q t$

where the coefficients $a_{p q}$ may be computed explicitly. Therefore, to determine $w^{(4)}$ we must check that the compatibility condition $f_{4}^{(2)}=0$ is satisfied for coefficients of the right hand side, i.e. $a_{21}=0$. This is independent of $A^{(3)}$. Indeed, the coefficients involving $\cos 4 x$ on the right-hand side come from

$$
2 \mathcal{N}_{2}\left(w^{(1)}, w_{3}^{(3)}\right)+\mathcal{N}_{2}\left(w_{2}^{(2)}, w_{2}^{(2)}\right)+3 \mathcal{N}_{3}\left(w^{(1)}, w^{(1)}, w_{2}^{(2)}\right)+\mathcal{N}_{4}\left(\left\{w^{(1)}\right\}^{(4)}\right)
$$

where $w_{2}^{(2)}$ and $w_{3}^{(3)}$ are the terms involving $\cos 2 x$ and $\cos 3 x$ respectively of $w^{(2)}$ and $w^{(3)}$. It can be checked that these terms have no term in $\cos 4 x \cos 2 t$. Indeed

$$
\begin{aligned}
2 \mathcal{N}_{2}\left(w^{(1)}, w_{3}^{(3)}\right)= & \frac{9}{8}(1-\cos 4 t)(4 \cos 4 x-\cos 2 x)+ \\
& +3 \cos 2 x(\cos 2 t+\cos 4 t), \\
\mathcal{N}_{2}\left(w_{2}^{(2)}, w_{2}^{(2)}\right)= & 2 \cos 4 x(1-\cos 4 t)+2(\cos 2 t+\cos 4 t), \\
3 \mathcal{N}_{3}\left(w^{(1)}, w^{(1)}, w_{2}^{(2)}\right)= & (1-\cos 4 t)(2 \cos 2 x-3 \cos 4 x), \\
\mathcal{N}_{4}\left(\left\{w^{(1)}\right\}^{(4)}\right)= & \frac{1}{4}(1-\cos 4 t)(\cos 4 x-\cos 2 x),
\end{aligned}
$$

which shows that the required compatibility condition is satisfied. The result is that

$$
w^{(4)}=A^{(4)} \cos 4 x \cos 2 t+2 A^{(3)} w^{(2)}+\widetilde{w}^{(4)}
$$

where $\widetilde{w}^{(4)}$ is known and orthogonal to $\operatorname{ker}\left(\mathcal{L}_{1}\right)$, and $A^{(4)}$ is still unknown. The computation of $w^{(5)}$ leads to a compatibility condition of the form $f_{1}^{(1)}=0$ 
which must be satisfied. This ensures that we can determine $A^{(3)}$; in fact it is straightforward (but not done here) to show that $A^{(3)}=-37 / 32$. (The theoretical reason for these compatibility conditions to always hold, and an algorithm for calculating approximate solutions to all orders, is given in [1].) Notice that if an approximate solution of order $\varepsilon^{3}$ is all that is needed, it is not necessary to calculate $A^{(3)}$ since, in the expression $\mathcal{F}\left(w_{\varepsilon}^{(3)}, 1+\varepsilon^{2} / 4\right)$, the coefficient $A^{(3)}$ appears at order $\varepsilon^{4}$.

\section{Proof of Proposition 5.2}

For convenience with notation, from now on we suppress the suffix $w^{\prime}$ in the notation for the operators $L_{w^{\prime}}$, and $M_{w^{\prime}}$. First note, from Lemma 3.2, that

$$
\left[L^{-1} g, u\right]=g \mathcal{H}\left(\frac{1}{D} L u\right)-(L u) \mathcal{H}(g / D) .
$$

Now, from (5.12), (3.10), (3.2) and the fact that $-\mathcal{H}(L \dot{w})=\phi^{\prime}$, we obtain

$$
\begin{aligned}
\mathcal{Q} g= & \mathcal{H}\left(g \mathcal{H}\left(\frac{1}{D} L \dot{w}\right)\right)-\mathcal{H}(L \dot{w} \mathcal{H}(g / D))+\frac{1}{D} \mathcal{H}(L \dot{w} \mathcal{H} g)+ \\
& +(\mathcal{H} g) \mathcal{H}\left(\frac{1}{D} L \dot{w}\right)-\frac{1}{D} \mathcal{H}\left(\phi^{\prime} g\right)-\phi^{\prime} \mathcal{H}(g / D) \\
= & \mathcal{H}(a g)-\left\{\mathcal{H}\left(\frac{g}{D} \mathcal{H} L \dot{w}\right)+\mathcal{H}(L \dot{w} \mathcal{H}(g / D))+\phi^{\prime} \mathcal{H}(g / D)\right\}+ \\
& +a \mathcal{H} g-\frac{1}{D}\left\{\mathcal{H}(L \dot{w}) \mathcal{H} g-\mathcal{H}(L \dot{w} \mathcal{H} g)+\mathcal{H}\left(\phi^{\prime} g\right)\right\} \\
= & \mathcal{H}(a g)+a \mathcal{H} g+\left(\frac{\pi_{0}(g)}{D}-\pi_{0}\left(\frac{g}{D}\right)\right) \pi_{0}\left(L_{w^{\prime}} \dot{w}\right) \\
= & J(a, g)+\left(\frac{\pi_{0}(g)}{D}-\pi_{0}\left(\frac{g}{D}\right)\right) \int_{0}^{t} \pi_{0}(\mathcal{F}(w, \mu))(\tau) d \tau,
\end{aligned}
$$

since, by (4.1) and the oddness in $t$ of $\pi_{0}\left(L_{w^{\prime}} \dot{w}\right)$,

$$
\pi_{0}(L \dot{w})=\int_{0}^{t} \pi_{0}(\mathcal{F}(w, \mu))(\tau) d \tau .
$$

This proves the proposition.

\section{Proof of Proposition 5.3}

From (3.10) and (5.13), the expression for $\mathcal{R}$ involves the two terms

$$
\begin{aligned}
M^{-1} J\left(M^{-1} J\left(\phi^{\prime}, \dot{w}\right), L^{-1} g\right) & =\frac{1}{D} \mathcal{H}\left(g M^{-1} J\left(\phi^{\prime}, \dot{w}\right)\right)+\mathcal{H}\left(\frac{g}{D}\right) M^{-1} J\left(\phi^{\prime}, \dot{w}\right), \\
M^{-1} J\left(\mathcal{H}\left[L^{-1} g, \dot{w}\right], \dot{w}\right) & =\frac{1}{D} \mathcal{H}\left(L \dot{w} \mathcal{H}\left[L^{-1} g, \dot{w}\right]\right)+\mathcal{H}\left(\frac{L \dot{w}}{D}\right) \mathcal{H}\left[L^{-1} g, \dot{w}\right],
\end{aligned}
$$


and since $\mathcal{H} L \dot{w}=-\phi^{\prime}$,

$$
\mathcal{H}\left(\frac{L \dot{w}}{D}\right)=a+\frac{\phi^{\prime}}{D}
$$

We also have, from (C.1), (3.2) and (3.10),

$$
\begin{aligned}
\mathcal{H}\left[L^{-1} g, \dot{w}\right] & =\mathcal{H}(a g)+\phi^{\prime} \mathcal{H}\left(\frac{g}{D}\right)+\frac{g \mathcal{H} \phi^{\prime}}{D}+\left(\frac{g}{D}-\pi_{0}\left(\frac{g}{D}\right)\right) \pi_{0}(L \dot{w}), \\
M^{-1} J\left(\phi^{\prime}, \dot{w}\right) & =a \phi^{\prime}+\frac{\phi^{\prime 2}}{D}+\frac{1}{D} \mathcal{H}\left(\phi^{\prime} \mathcal{H} \phi^{\prime}\right)+\frac{\mathcal{H} \phi^{\prime}}{D} \pi_{0}(L \dot{w}) .
\end{aligned}
$$

First we collect the terms in expression (5.13) for $\mathcal{R}$ which involve $\pi_{0}(L \dot{w})$. After expansion and simplification using (3.2) these terms reduce to

$$
\left(a \pi_{0}\left(\frac{g}{D}\right)-\frac{\pi_{0}(a g)}{D}\right)\left(\pi_{0}(L \dot{w})\right)-\frac{1}{D} \mathcal{H}\left(\frac{g}{D}\right)\left(\pi_{0}(L \dot{w})\right)^{2} .
$$

Now the remaining terms in the expression of $\mathcal{R}$ can be written

$$
\begin{aligned}
& \frac{1}{D} \mathcal{H}\left\{g\left(a \phi^{\prime}+\frac{\phi^{\prime 2}}{D}+\frac{1}{D} \mathcal{H}\left(\phi^{\prime} \mathcal{H} \phi^{\prime}\right)\right)-\left(\mathcal{H} \phi^{\prime}\right)\left(\mathcal{H}(a g)+\phi^{\prime} \mathcal{H}\left(\frac{g}{D}\right)+\frac{g \mathcal{H} \phi^{\prime}}{D}\right)\right\} \\
& +\mathcal{H}\left(\frac{g}{D}\right)\left\{a \phi^{\prime}+\frac{\phi^{\prime 2}}{D}+\frac{1}{D} \mathcal{H}\left(\phi^{\prime} \mathcal{H} \phi^{\prime}\right)\right\}-\left(a+\frac{\phi^{\prime}}{D}\right)\left\{\mathcal{H}(a g)+\phi^{\prime} \mathcal{H}\left(\frac{g}{D}\right)+\frac{g \mathcal{H} \phi^{\prime}}{D}\right\} \\
& =\frac{1}{D} \mathcal{H}\left\{g a \phi^{\prime}-\frac{g}{D} \mathcal{H}\left(\phi^{\prime} \mathcal{H} \phi^{\prime}\right)-\left(\mathcal{H} \phi^{\prime}\right) \mathcal{H}(a g)-\left(\phi^{\prime} \mathcal{H} \phi^{\prime}\right) \mathcal{H}\left(\frac{g}{D}\right)\right\} \\
& +\frac{1}{D} \mathcal{H}\left(\frac{g}{D}\right) \mathcal{H}\left(\phi^{\prime} \mathcal{H} \phi^{\prime}\right)-\left(a+\frac{\phi^{\prime}}{D}\right)\left\{\mathcal{H}(a g)+\frac{g \mathcal{H} \phi^{\prime}}{D}\right\} .
\end{aligned}
$$

Using again (3.2) and the fact that

$$
\pi_{0}\left(\phi^{\prime} \mathcal{H} \phi^{\prime}\right)=0
$$

this simplifies to

$$
\frac{1}{D} \mathcal{H}\left\{g a \phi^{\prime}-\left(\mathcal{H} \phi^{\prime}\right)(\mathcal{H}(a g)\}-\left(a+\frac{\phi^{\prime}}{D}\right) \mathcal{H}(a g)-a g \frac{\mathcal{H} \phi^{\prime}}{D}\right.
$$

and, since $\mathcal{H}$ acting on (3.2) yields

$$
\mathcal{H}\left\{g a \phi^{\prime}-\left(\mathcal{H} \phi^{\prime}\right)(\mathcal{H}(a g)\}=a g \mathcal{H} \phi^{\prime}+\phi^{\prime} \mathcal{H}(a g),\right.
$$

we finally obtain an expression for the terms in $\mathcal{R}$ that do not involve $\pi_{0}(L \dot{w})$,

$$
-a \mathcal{H}(a g) .
$$

Combining these calculations with (C.2) proves the proposition. 


\section{E Proof of Proposition 5.4}

This appendix gives the precise form of $a_{1}, a_{2}, a_{3}$. We first notice that in addition to

$$
a=\mathcal{H}\left(\frac{1}{D} L \dot{w}\right)+\frac{1}{D} \mathcal{H}(L \dot{w}) \in H_{\text {吅 }}^{m-1, o o},
$$

we have, from Lemma 3.1,

$$
\begin{aligned}
\partial_{t}\left(\frac{1}{D}\right) & =-\frac{2}{D} \mathcal{H}\left(\frac{L \dot{w}^{\prime}}{D}\right), & \partial_{x}\left(\frac{1}{D}\right) & =-\frac{2}{D} \mathcal{H}\left(\frac{L w^{\prime \prime}}{D}\right) \\
\partial_{t}(L \dot{w}) & =L \ddot{w}+\left[\dot{w}, \dot{w}^{\prime}\right], & \partial_{x}(L \dot{w}) & =L \dot{w}^{\prime}+\left[\dot{w}, w^{\prime \prime}\right] .
\end{aligned}
$$

Hence

$$
\begin{aligned}
a^{\prime}= & \mathcal{H}\left(\frac{L \dot{w}^{\prime}}{D}\right)+\frac{1}{D} \mathcal{H}\left(L \dot{w}^{\prime}\right)+\mathcal{H}\left(\frac{\left[\dot{w}, w^{\prime \prime}\right]}{D}\right)+\frac{1}{D} \mathcal{H}\left[\dot{w}, w^{\prime \prime}\right]+ \\
& -\frac{2}{D}(\mathcal{H} L \dot{w}) \mathcal{H}\left(\frac{L w^{\prime \prime}}{D}\right)-2 \mathcal{H}\left\{\frac{L \dot{w}}{D} \mathcal{H}\left(\frac{L w^{\prime \prime}}{D}\right)\right\}, \\
\dot{a}= & \mathcal{H}\left(\frac{L \ddot{w}}{D}\right)+\mathcal{H}\left(\frac{\left[\dot{w}, \dot{w}^{\prime}\right]}{D}\right)+\frac{1}{D} \mathcal{H}(L \ddot{w})+\frac{1}{D} \mathcal{H}\left[\dot{w}, \dot{w}^{\prime}\right]+ \\
& -2 \mathcal{H}\left\{\frac{L \dot{w}}{D} \mathcal{H}\left(\frac{L \dot{w}^{\prime}}{D}\right)\right\}-\frac{2}{D}(\mathcal{H} L \dot{w}) \mathcal{H}\left(\frac{L \dot{w}^{\prime}}{D}\right) .
\end{aligned}
$$

From Lemma 3.2 and the preceding formulae for $a^{\prime}$ and $\dot{a}$,

$$
\begin{aligned}
a^{\prime} & =\mathcal{H}\left(\frac{L \dot{w}^{\prime}}{D}\right)+\frac{1}{D} \mathcal{H}\left(L \dot{w}^{\prime}\right)-\mathcal{H}\left\{\frac{L w^{\prime \prime}}{D} \mathcal{H}\left(\frac{L \dot{w}}{D}\right)\right\}+\frac{1}{D} \mathcal{H}\left\{L \dot{w} \mathcal{H}\left(\frac{L w^{\prime \prime}}{D}\right)\right\} \\
& -\frac{1}{D} \mathcal{H}\left\{L w^{\prime \prime} \mathcal{H}\left(\frac{L \dot{w}}{D}\right)\right\}-\frac{2}{D}(\mathcal{H} L \dot{w}) \mathcal{H}\left(\frac{L w^{\prime \prime}}{D}\right)-\mathcal{H}\left\{\frac{L \dot{w}}{D} \mathcal{H}\left(\frac{L w^{\prime \prime}}{D}\right)\right\},
\end{aligned}
$$

and

$$
\begin{aligned}
\dot{a}= & \mathcal{H}\left(\frac{L \ddot{w}}{D}\right)+\frac{1}{D} \mathcal{H}(L \ddot{w})-\mathcal{H}\left\{\frac{L \dot{w}}{D} \mathcal{H}\left(\frac{L \dot{w}^{\prime}}{D}\right)\right\}-\mathcal{H}\left\{\frac{L \dot{w}^{\prime}}{D} \mathcal{H}\left(\frac{L \dot{w}}{D}\right)\right\}+ \\
& +\frac{1}{D} \mathcal{H}\left\{L \dot{w} \mathcal{H}\left(\frac{L \dot{w}^{\prime}}{D}\right)\right\}-\frac{1}{D} \mathcal{H}\left\{L \dot{w}^{\prime} \mathcal{H}\left(\frac{L \dot{w}}{D}\right)\right\}-\frac{2}{D}(\mathcal{H} L \dot{w}) \mathcal{H}\left(\frac{L \dot{w}^{\prime}}{D}\right) .
\end{aligned}
$$

Now applying identity $(3.2)$, since $\pi_{0}\left(L w^{\prime \prime} / D\right)=\pi_{0} w^{\prime \prime}=0$, and $\pi_{0}\left(L \dot{w}^{\prime} / D\right)=$ $\pi_{0} \dot{w}^{\prime}=0($ see $(\mathrm{A} .1))$ we obtain

$$
\begin{aligned}
a^{\prime}= & \mathcal{H}\left(\frac{L \dot{w}^{\prime}}{D}\right)+\frac{1}{D} \mathcal{H}\left(L \dot{w}^{\prime}\right)-\mathcal{H}\left(\frac{L w^{\prime \prime}}{D}\right) \mathcal{H}\left(\frac{L \dot{w}}{D}\right)-\frac{2}{D} \mathcal{H}(L \dot{w}) \mathcal{H}\left(\frac{L w^{\prime \prime}}{D}\right) \\
& +\frac{L w^{\prime \prime}}{D} \frac{L \dot{w}}{D}+\frac{1}{D} \mathcal{H}\left\{L \dot{w} \mathcal{H}\left(\frac{L w^{\prime \prime}}{D}\right)\right\}-\frac{1}{D} \mathcal{H}\left\{L w^{\prime \prime} \mathcal{H}\left(\frac{L \dot{w}}{D}\right)\right\}
\end{aligned}
$$

and

$$
\begin{aligned}
\dot{a}= & \mathcal{H}\left(\frac{L \ddot{w}}{D}\right)+\frac{1}{D} \mathcal{H}(L \ddot{w})-\mathcal{H}\left(\frac{L \dot{w}}{D}\right) \mathcal{H}\left(\frac{L \dot{w}^{\prime}}{D}\right)+\frac{L \dot{w}}{D} \frac{L \dot{w}^{\prime}}{D}+ \\
& +\frac{1}{D} \mathcal{H}\left\{L \dot{w} \mathcal{H}\left(\frac{L \dot{w}^{\prime}}{D}\right)\right\}-\frac{1}{D} \mathcal{H}\left\{L \dot{w}^{\prime} \mathcal{H}\left(\frac{L \dot{w}}{D}\right)\right\}-\frac{2}{D} \mathcal{H}(L \dot{w}) \mathcal{H}\left(\frac{L \dot{w}^{\prime}}{D}\right) .
\end{aligned}
$$


Recall from (5.17) that

$$
\begin{aligned}
& a_{1}=a \mathcal{H}\left(\frac{L w^{\prime \prime}}{D}\right)-\mathcal{H}\left(\frac{L \dot{w}^{\prime}}{D}\right)-D^{-1} \mathcal{H}\left(L \dot{w}^{\prime}-a L w^{\prime \prime}\right), \\
& a_{2}=\mathcal{H}\left(\frac{L \ddot{w}}{D}\right)-a \mathcal{H}\left(\frac{L \dot{w}^{\prime}}{D}\right)-D^{-1} \mathcal{H}\left(a L \dot{w}^{\prime}-L \ddot{w}\right),
\end{aligned}
$$

and hence

$$
\begin{aligned}
-a_{1}= & \mathcal{H}\left(\frac{L \dot{w}^{\prime}}{D}\right)+\frac{1}{D} \mathcal{H}\left(L \dot{w}^{\prime}\right)-\frac{1}{D} \mathcal{H}\left\{L w^{\prime \prime} \mathcal{H}\left(\frac{L \dot{w}}{D}\right)\right\} \\
& -\frac{1}{D} \mathcal{H}\left\{\frac{L w^{\prime \prime}}{D} \mathcal{H}(L \dot{w})\right\}-\frac{1}{D} \mathcal{H}(L \dot{w}) \mathcal{H}\left(\frac{L w^{\prime \prime}}{D}\right)-\mathcal{H}\left(\frac{L \dot{w}}{D}\right) \mathcal{H}\left(\frac{L w^{\prime \prime}}{D}\right)
\end{aligned}
$$

and, by (3.2),

$$
\begin{aligned}
a^{\prime}+a_{1}= & -\frac{1}{D} \mathcal{H}(L \dot{w}) \mathcal{H}\left(\frac{L w^{\prime \prime}}{D}\right)+\frac{L w^{\prime \prime}}{D} \frac{L \dot{w}}{D} \\
& +\frac{1}{D} \mathcal{H}\left\{L \dot{w} \mathcal{H}\left(\frac{L w^{\prime \prime}}{D}\right)\right\}+\frac{1}{D} \mathcal{H}\left\{\frac{L w^{\prime \prime}}{D} \mathcal{H}(L \dot{w})\right\}=0 .
\end{aligned}
$$

Also

$$
\begin{aligned}
a_{2}= & \mathcal{H}\left(\frac{L \ddot{w}}{D}\right)+\frac{1}{D} \mathcal{H}(L \ddot{w})-\mathcal{H}\left(\frac{L \dot{w}^{\prime}}{D}\right) \mathcal{H}\left(\frac{L \dot{w}}{D}\right)-\frac{1}{D} \mathcal{H}\left(\frac{L \dot{w}^{\prime}}{D}\right) \mathcal{H}(L \dot{w}) \\
& -\frac{1}{D} \mathcal{H}\left\{L \dot{w}^{\prime} \mathcal{H}\left(\frac{L \dot{w}}{D}\right)\right\}-\frac{1}{D} \mathcal{H}\left\{\frac{L \dot{w}^{\prime}}{D} \mathcal{H}(L \dot{w})\right\},
\end{aligned}
$$

hence, again by (3.2),

$$
\begin{aligned}
\dot{a}-a_{2}= & \frac{L \dot{w}}{D} \frac{L \dot{w}^{\prime}}{D}-\frac{1}{D} \mathcal{H}\left(\frac{L \dot{w}^{\prime}}{D}\right) \mathcal{H}(L \dot{w})+\frac{1}{D} \mathcal{H}\left\{\frac{L \dot{w}^{\prime}}{D} \mathcal{H}(L \dot{w})\right\} \\
& +\frac{1}{D} \mathcal{H}\left\{L \dot{w} \mathcal{H}\left(\frac{L \dot{w}^{\prime}}{D}\right)\right\}=0 .
\end{aligned}
$$

Recall from (5.16) and (5.17) that

$$
a_{3}=a \mathcal{H}\left(L \dot{w}^{\prime}-a L w^{\prime \prime}\right)+\mathcal{H}\left(a L \dot{w}^{\prime}-L \ddot{w}\right)-\mu w^{\prime}-\frac{\left(\pi_{0} L \dot{w}\right)^{2}}{D} \mathcal{H}\left(\frac{L w^{\prime \prime}}{D}\right),
$$

and note, from the definition of $\mathcal{F}(w, \mu)$, that

$$
\mu w^{\prime}+\mathcal{H} \partial_{t} L \dot{w}=\mathcal{H}(\mathcal{F}(w, \mu))+\partial_{x} M^{-1} J(\mathcal{H} L \dot{w}, \dot{w}) .
$$

Therefore, by (3.10),

$$
\begin{aligned}
\mu w^{\prime}+\mathcal{H} L \ddot{w} & =\mathcal{H}(\mathcal{F}(w, \mu))-\mathcal{H}\left(\left[\dot{w}, \dot{w}^{\prime}\right]\right)-\frac{2}{D} \mathcal{H}\left(\frac{L w^{\prime \prime}}{D}\right) \mathcal{H}(L \dot{w} \mathcal{H} L \dot{w} \\
& +\frac{1}{D} \mathcal{H}\left(L \dot{w}^{\prime} \mathcal{H} L \dot{w}\right)+\frac{1}{D} \mathcal{H}\left(\left[\dot{w}, w^{\prime \prime}\right] \mathcal{H} L \dot{w}\right)+\frac{1}{D} \mathcal{H}\left(L \dot{w} \mathcal{H} L \dot{w}^{\prime}\right. \\
& +\frac{1}{D} \mathcal{H}\left(L \dot{w} \mathcal{H}\left[\dot{w}, w^{\prime \prime}\right]\right)+\left(\mathcal{H} L \dot{w}^{\prime}\right) \mathcal{H}\left(\frac{L \dot{w}}{D}\right)+\left(\mathcal{H}\left[\dot{w}, w^{\prime \prime}\right]\right) \mathcal{H}\left(\frac{L \dot{w}}{D}\right) \\
& +\mathcal{H} L \dot{w}\left\{\mathcal{H}\left(\frac{L \dot{w}^{\prime}}{D}\right)+\mathcal{H}\left(\frac{\left[\dot{w}, w^{\prime \prime}\right]}{D}\right)\right\}-2(\mathcal{H} L \dot{w}) \mathcal{H}\left(\frac{L \dot{w}}{D} \mathcal{H}\left(\frac{L w^{\prime \prime}}{D}\right)\right) .
\end{aligned}
$$


In addition to $-\mathcal{H} \mathcal{F}(w, \mu), a_{3}$ involves (i) terms which involve $\dot{w}^{\prime}$, and (ii) terms which involve $w^{\prime \prime}$. We will see that both contribute zero. i) Terms with $\dot{w}^{\prime}$ :

$$
\begin{aligned}
& \left(\mathcal{H} L \dot{w}^{\prime}\right)\left\{\mathcal{H}\left(\frac{L \dot{w}}{D}\right)+\frac{1}{D} \mathcal{H} L \dot{w}\right\}+\mathcal{H}\left(L \dot{w}^{\prime} \mathcal{H} \frac{L \dot{w}}{D}\right)+\mathcal{H}\left(\frac{L \dot{w}^{\prime}}{D} \mathcal{H} L \dot{w}\right)+\mathcal{H}\left(\left[\dot{w}, \dot{w}^{\prime}\right]\right) \\
& -\frac{1}{D} \mathcal{H}\left(L \dot{w}^{\prime} \mathcal{H} L \dot{w}\right)-\frac{1}{D} \mathcal{H}\left(L \dot{w} \mathcal{H} L \dot{w}^{\prime}\right)-\left(\mathcal{H} L \dot{w}^{\prime}\right) \mathcal{H}\left(\frac{L \dot{w}}{D}\right)-(\mathcal{H} L \dot{w}) \mathcal{H}\left(\frac{L \dot{w}^{\prime}}{D}\right)
\end{aligned}
$$

which, by Lemma 3.2,

$$
\begin{gathered}
=\frac{1}{D}\left(\mathcal{H} L \dot{w}^{\prime}\right)(\mathcal{H} L \dot{w})+\mathcal{H}\left(\frac{L \dot{w}^{\prime}}{D} \mathcal{H} L \dot{w}\right)+\mathcal{H}\left\{L \dot{w} \mathcal{H}\left(\frac{L \dot{w}^{\prime}}{D}\right)\right\} \\
-\frac{1}{D} \mathcal{H}\left(L \dot{w}^{\prime} \mathcal{H} L \dot{w}\right)-\frac{1}{D} \mathcal{H}\left(L \dot{w} \mathcal{H} L \dot{w}^{\prime}\right)-(\mathcal{H} L \dot{w}) \mathcal{H}\left(\frac{L \dot{w}^{\prime}}{D}\right) \\
=-\frac{L \dot{w}^{\prime}}{D} L \dot{w}+\pi_{0}\left(\frac{L \dot{w}^{\prime}}{D}\right) \pi_{0}(L \dot{w})+\frac{1}{D} L \dot{w}^{\prime} L \dot{w}-\frac{1}{D} \pi_{0}\left(L \dot{w}^{\prime}\right) \pi_{0}(L \dot{w})=0
\end{gathered}
$$

since

$$
\pi_{0}\left(L \dot{w}^{\prime}\right)=0\left(\dot{w}^{\prime} \text { odd }\right) \text { and } \pi_{0}\left(\frac{L \dot{w}^{\prime}}{D}\right)=\pi_{0}\left(\dot{w}^{\prime}\right)=0(\text { see }(\text { A.1 })) .
$$

ii) Terms with $w^{\prime \prime}$ :

$$
\begin{aligned}
& -a \mathcal{H}\left(a L w^{\prime \prime}\right)+\frac{2}{D} \mathcal{H}\left(\frac{L w^{\prime \prime}}{D}\right) \mathcal{H}(L \dot{w} \mathcal{H} L \dot{w})-\frac{1}{D} \mathcal{H}\left(\left[\dot{w}, w^{\prime \prime}\right] \mathcal{H} L \dot{w}\right) \\
& -\frac{1}{D} \mathcal{H}\left(L \dot{w} \mathcal{H}\left[\dot{w}, w^{\prime \prime}\right]\right)-\left(\mathcal{H}\left[\dot{w}, w^{\prime \prime}\right]\right) \mathcal{H}\left(\frac{L \dot{w}}{D}\right)-\mathcal{H}\left(\frac{\left[\dot{w}, w^{\prime \prime}\right]}{D}\right) \mathcal{H} L \dot{w} \\
& +2(\mathcal{H} L \dot{w}) \mathcal{H}\left(\frac{L \dot{w}}{D} \mathcal{H}\left(\frac{L w^{\prime \prime}}{D}\right)\right)-\frac{\left(\pi_{0}(L \dot{w})\right)^{2}}{D} \mathcal{H}\left(\frac{L w^{\prime \prime}}{D}\right)
\end{aligned}
$$

which, with Lemma 3.2 and (3.2), gives

$$
\begin{aligned}
&=-a \mathcal{H}\left(a L w^{\prime \prime}+\left[\dot{w}, w^{\prime \prime}\right]\right)+\frac{1}{D} \mathcal{H}\left(\frac{L w^{\prime \prime}}{D}\right)\left\{(\mathcal{H} L \dot{w})^{2}+\left(\pi_{0} L \dot{w}\right)^{2}\right\}+ \\
&-\frac{L \dot{w}}{D} L w^{\prime \prime} \mathcal{H}\left(\frac{L \dot{w}}{D}\right)-(\mathcal{H} L \dot{w})\left\{\mathcal{H}\left(\frac{L \dot{w}}{D} \mathcal{H}\left(\frac{L w^{\prime \prime}}{D}\right)\right)-\mathcal{H}\left(\frac{L w^{\prime \prime}}{D} \mathcal{H}\left(\frac{L \dot{w}}{D}\right)\right)\right\} \\
&+2(\mathcal{H} L \dot{w}) \mathcal{H}\left(\frac{L \dot{w}}{D} \mathcal{H}\left(\frac{L w^{\prime \prime}}{D}\right)\right)-\frac{\left(\pi_{0}(L \dot{w})\right)^{2}}{D} \mathcal{H}\left(\frac{L w^{\prime \prime}}{D}\right) \\
&=-\left\{\mathcal{H}\left(\frac{L \dot{w}}{D}\right)+\frac{1}{D} \mathcal{H}(L \dot{w})\right\}\left\{(\mathcal{H} L \dot{w}) \mathcal{H}\left(\frac{L w^{\prime \prime}}{D}\right)-L \dot{w} \frac{L w^{\prime \prime}}{D}\right\}+ \\
&+\frac{1}{D} \mathcal{H}\left(\frac{L w^{\prime \prime}}{D}\right)(\mathcal{H} L \dot{w})^{2}-\frac{L \dot{w}}{D} L w^{\prime \prime} \mathcal{H}\left(\frac{L \dot{w}}{D}\right)+ \\
&+(\mathcal{H} L \dot{w})\left\{\mathcal{H}\left(\frac{L \dot{w}}{D}\right) \mathcal{H}\left(\frac{L w^{\prime \prime}}{D}\right)-\frac{L \dot{w}}{D} \frac{L w^{\prime \prime}}{D}\right\}
\end{aligned}
$$

since $\pi_{0}\left[\dot{w}, w^{\prime \prime}\right]=0$ because of the oddness, and $\pi_{0}\left(\left(L w^{\prime \prime} / D\right)\right)=0$ (see (A.1)). Hence all terms involving $w^{\prime \prime}$ and $\dot{w}^{\prime}$ contribute zero,

$$
a_{3}=-\mathcal{H}(\mathcal{F}(w, \mu))
$$

and the Proposition 5.4 is proved. 


\section{F Proof of Lemma 5.1 Concluded}

In this appendix we compute, up to order $O\left(\varepsilon^{2}\right)$, the coefficients $a$ and $b$ of the Lemma 5.1. We start with

$$
w=\varepsilon \cos x \cos t+\frac{\varepsilon^{2}}{4}\{\cos 2 t-2 \cos 2 x(1+\cos 2 t)\}+O\left(\varepsilon^{3}\right),
$$

which leads to

$$
\begin{aligned}
D & =1-2 \varepsilon \cos x \cos t+\varepsilon^{2}(1+\cos 2 t)(2 \cos 2 x+1 / 2)+O\left(\varepsilon^{3}\right), \\
D^{-1} & =1+2 \varepsilon \cos x \cos t+\varepsilon^{2}(1+\cos 2 t)(1 / 2-\cos 2 x)+O\left(\varepsilon^{3}\right), \\
\mathcal{H} L \dot{w} & =\varepsilon \sin x \sin t-\varepsilon^{2} \sin 2 x \sin 2 t+O\left(\varepsilon^{3}\right), \\
D^{-1} \mathcal{H} L \dot{w} & =\varepsilon \sin x \sin t-\frac{1}{2} \varepsilon^{2} \sin 2 x \sin 2 t+O\left(\varepsilon^{3}\right), \\
\mathcal{H}\left(\frac{L \dot{w}}{D}\right) & =\varepsilon \sin x \sin t-\frac{1}{2} \varepsilon^{2} \sin 2 x \sin 2 t+O\left(\varepsilon^{3}\right), \\
a & =2 \varepsilon \sin x \sin t-\varepsilon^{2} \sin 2 x \sin 2 t+O\left(\varepsilon^{3}\right), \\
L \ddot{w} & =-\varepsilon \cos x \cos t+\varepsilon^{2}\left\{\frac{1}{2}(1-\cos 2 t)+2 \cos 2 x \cos 2 t\right\}+O\left(\varepsilon^{3}\right) \\
L \dot{w}^{\prime} & =\varepsilon \sin x \sin t-2 \varepsilon^{2} \sin 2 x \sin 2 t+O\left(\varepsilon^{3}\right) \\
L w^{\prime \prime} & =-\varepsilon \cos x \cos t+\varepsilon^{2}\left(\frac{1}{2}+2 \cos 2 x\right)(1+\cos 2 t)+O\left(\varepsilon^{3}\right) \\
L \dot{w}^{\prime}-a L w^{\prime \prime} & =\varepsilon \sin x \sin t-\frac{3}{2} \varepsilon^{2} \sin 2 x \sin 2 t+O\left(\varepsilon^{3}\right), \\
a L \dot{w}^{\prime}-L \ddot{w} & =\varepsilon \cos x \cos t-\frac{1}{2} \varepsilon^{2} \cos 2 x(1+3 \cos 2 t)+O\left(\varepsilon^{3}\right),
\end{aligned}
$$

Therefore

$$
b=-2 \varepsilon \cos x \cos t+\varepsilon^{2}(-1+\cos 2 x(1+\cos 2 t))+O\left(\varepsilon^{3}\right),
$$

and, since $a_{3}=-\mathcal{H} \mathcal{F}(w, \mu)=O\left(\varepsilon^{3}\right)$ when $w$ is an approximate solution at order $\varepsilon^{2}$, the lemma is proved.

\section{G Changes of Variables}

There follows two lemmas on composition estimates are central to the analysis. In [12, Appendix] they are proved for spaces of Hölder continuous functions but their proofs in the $C^{k}$-spaces of continuous periodic functions are the same.

Lemma G.1. For $k>0$ let $D_{k}=\left\{p \in \mathbb{R}^{d}:\|p\| \leq k\right\}$ and suppose $\boldsymbol{\varphi}: \Omega \rightarrow D_{k}$, $f: D_{k} \rightarrow \mathbb{R}$. Then, for any $s \in \mathbb{N}_{0}$, there exists a constant $c$, depending only on $s$ and $k$, such that

$$
\|f \circ \varphi\|_{C^{s}(\Omega)} \leq c\left(\|f\|_{C^{s}\left(D_{k}\right)}\|\varphi\|_{C^{1}(\Omega)}^{s}+\|f\|_{C^{1}\left(D_{k}\right)}\|\varphi\|_{C^{s}(\Omega)}+\|f\|_{C\left(D_{k}\right)}\right) .
$$


In particular, for any functions $v_{i} \in C^{s}(\Omega), i=1,2$,

$$
\left\|v_{1} v_{2}\right\|_{C^{s}(\Omega)} \leq c\left(\left\|v_{1}\right\|_{C^{s}(\Omega)}\left\|v_{2}\right\|_{C(\Omega)}+\left\|v_{2}\right\|_{C^{s}(\Omega)}\left\|v_{1}\right\|_{C(\Omega)}\right) .
$$

Lemma G.2. Let $I+g: \mathbb{R}^{2} \rightarrow \mathbb{R}^{2}$, where $g$ is $2 \pi$-periodic in $x$ and $t$, be a $C^{1}$-diffeomorphism with inverse $I+f$. Suppose also that $\|f\|_{C^{1}}+\|g\|_{C^{1}} \leq M$. Then if $f$ or $g$ is in $C^{k}$

$$
\|1+f\|_{C^{k}} \leq c_{k}(M)\|1+g\|_{C^{k}}
$$

A similar result to Lemma G.1 holds when $f \in H^{s}$ and $\varphi \in C^{k}$.

Lemma G.3. Let $f \in H_{\text {如 }}^{s}$ for $s \in \mathbb{N}$ and let $\varphi$ be a $2 \pi$-periodic $C^{s}$-perturbation of the identity on $\mathbb{R}^{2}$. Then, for any $s \in \mathbb{N}_{0}$, there exists a constant $c$, depending only on s, such that

$$
\|f \circ \varphi\|_{s} \leq c\left(\|f\|_{s}\|\varphi\|_{C^{1}(\Omega)}^{s}+\|f\|_{1}\|\varphi\|_{C^{s}(\Omega)}+\|f\|_{0}\right) .
$$

Let $w \in H^{4}$ so that $a \in C^{1}$. To solve equation (6.10) using characteristics let $u(x, t)$ be the unique solution of the initial value problem

$$
\dot{u}(x, t)+a(x+u(x, t), t)=0, \quad u(x, 0)=0 .
$$

Since $\|a\|_{C^{1}} \leq c_{1}\left(M_{3}\right)\|w\|_{4}$ (see (5.3)) it follows that $(I+f)(x, t):=(x, t)+$ $(u(x, t), 0)$ is a $C^{1}$-diffeomorphism close to the identity when $\|w\|_{4}$ is sufficiently small. If its inverse is $(I+g)(x, t)=:(x, t)+(d(x, t), 0)$, then $d \in C^{1}$ satisfies (6.10). Moreover,

$$
d(x, t)=-u((I+g)(x, t), t),
$$

and hence, from Lemmas G.1 and G.2,

$$
\|d\|_{C^{s}} \leq c_{s}\left(M_{3}\right)\left(\|u\|_{C^{s}}\|I+f\|_{C^{1}}^{s}+\|u\|_{C^{1}}\|1+f\|_{C^{s}}+\|u\|_{C^{0}}\right) .
$$

Since $f(x, t)=(u(x, t), 0)$ it remains to find bounds on $\|u\|_{s}$ in terms of $\|w\|_{s}$. It follows from (G.1) and Lemma G.1 that

$$
\begin{aligned}
\left\|\frac{\partial^{k+l} u}{\partial^{k} t \partial^{l} x} \partial_{t} u\right\|_{C^{0}} & \leq c_{k+l}\left(\|a\|_{C^{k+l}}\|I+f\|_{C^{1}}^{k+l}+\|a\|_{C^{1}}\|1+f\|_{C^{k+l}}+\|a\|_{C^{0}}\right) \\
& \leq c_{k+l}\left(M_{4}\right)\left(\|a\|_{C^{k+l}}+\|a\|_{C^{1}}\left(1+\|u\|_{C^{k+l}}\right)\right) .
\end{aligned}
$$

Therefore

$$
\begin{aligned}
\|\dot{u}\|_{C^{s}} & \leq\|u\|_{C^{s}}+c_{s}\left(M_{4}\right)\left(\|a\|_{C^{s}}+\|a\|_{C^{1}}\left(1+\|u\|_{C^{s}}\right)\right) \\
& \leq\|u\|_{C^{s}}\left(c_{s}\left(M_{4}\right)\|a\|_{C^{1}}+1\right)+c_{s}\left(M_{4}\right)\left(\|a\|_{C^{s}}+\|a\|_{C^{1}}\right) \\
& \leq c_{s}\left(M_{4}\right)\left(\|u\|_{C^{s}}+\|w\|_{s+3}\right)
\end{aligned}
$$

by (5.3). For the pure $x$-derivatives of $u$ we use (G.1) and the identities

$$
\begin{aligned}
u(x, t) & =-\int_{0}^{t} a(x+u(x, \tau), \tau) d \tau, \\
u^{\prime}(x, t) & =-\int_{0}^{t} a^{\prime}(x+u(x, \tau), \tau)\left(1+u^{\prime}(x, \tau)\right) d \tau .
\end{aligned}
$$


Hence, from (5.3),

$$
\|u\|_{C^{0}} \leq c\left(M_{3}\right)\|w\|_{3}
$$

and then, from (5.3) and Lemmas G.1 and G.3,

$$
\begin{aligned}
\|u\|_{C^{1}} & \leq c\left(M_{4}\right), \\
\left\|u^{\prime}\right\|_{C^{s}} & \leq c_{s}\left(\left\|a^{\prime} \circ(I+f)\right\|_{C^{s}}\left\|1+u^{\prime}\right\|_{C^{0}}+\left\|a^{\prime}\right\|_{C^{0}}\left\|1+u^{\prime}\right\|_{C^{s}}\right), \\
& \leq c_{s}\left(M_{4}\right)\left\{\|w\|_{s+4}+\|w\|_{5}\left(1+\|u\|_{C^{s}}\right)+\|w\|_{4}\left\|u^{\prime}\right\|_{C^{s}}\right\} .
\end{aligned}
$$

Thus, for $M_{4}$ small enough $\left(c_{s}\left(M_{4}\right)\|w\|_{4}<1 / 2\right.$ is enough),

$$
\left\|u^{\prime}\right\|_{C^{s}} \leq c_{s}\left(M_{4}\right)\left\{\|w\|_{s+4}+\|w\|_{5}\left(1+\|u\|_{C^{s}}\right)\right\} .
$$

Combining this with the estimate for $\dot{u}$ we obtain, for $M_{4}$ small enough,

$$
\|u\|_{C^{s+1}} \leq c_{s}\left(M_{4}\right)\left\{\|w\|_{s+4}+\|w\|_{5}\left(1+\|u\|_{C^{s}}\right)\right\}
$$

and, by induction, for any $s \geq 1$ and $M_{4}$ small enough,

$$
\|u\|_{C^{s}} \leq c_{s}\left(M_{4}\right)\|w\|_{s+3} .
$$

Since $\tilde{d}=-u$, it follows from (G.2) and Lemma G.2 that, for $M_{4}$ sufficiently small,

$$
\|d\|_{C^{s}} \leq c_{s}\left(M_{4}\right)\|w\|_{s+3} \text { and }\|\tilde{d}\|_{C^{s}} \leq c_{s}\left(M_{4}\right)\|w\|_{s+3} .
$$

\section{H Proofs of Lemmas 6.3, 6.5 and Corollaries}

For $f \in L_{\natural}^{2}$ and $\omega \in H_{\natural}^{m}$ (periodic functions of one variable), it follows from (3.1) that for almost all $x$,

$$
\begin{aligned}
&\left(\mathcal{S}_{\omega} f\right)(x)=-\frac{1}{2 \pi} \int_{-\pi}^{\pi} \frac{(\omega(z)-\omega(x)) f(z) d z}{\tan } \frac{1}{2}(x-z) \\
&=\frac{1}{2 \pi} \int_{-\pi}^{\pi} \frac{(x-z) \int_{0}^{1} \omega^{\prime}(x+s(z-x)) f(z) d z}{\tan \frac{1}{2}(x-z)},
\end{aligned}
$$

which is no longer a principal-value integral. Since $\omega \in C_{\natural}^{m-1}, \mathcal{S}_{\omega} f \in C_{\natural}^{m-2}$

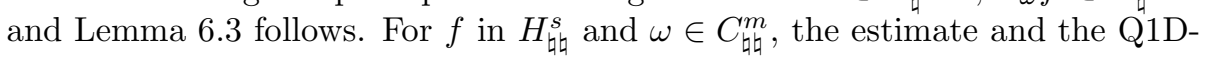
property of $\mathcal{S}_{\omega}$ in Lemma 6.5 follow from the above formula and Lemma G.1. The formulae for $\mathcal{S}_{\omega} \partial_{t} f$ and $\mathcal{S}_{\omega} \partial_{x} f$ follow from the properties of commutators of $\mathcal{H}$ and $\partial_{t}$ and $\partial_{x}$. The corresponding estimates in Corollary 6.6 for the Q1D operators $\mathcal{S}_{\dot{\omega}} f$ and $\mathcal{S}_{\omega} \partial_{x} f$ follow immediately from Lemma 6.5. To prove Corollary 6.7, we first observe from Lemma 6.5, that

$$
\left\|G_{0, \beta} \mathcal{S}_{\omega} f\right\|_{s} \leq c_{\beta, s}\left(M_{4}\right)\left(\|w\|_{\beta+s+r+1}\|f\|_{0}+\|w\|_{\beta+r+1}\|f\|_{s}\right) .
$$

Since $\partial_{y}\left(\left\{\mathcal{S}_{\omega} f\right\}^{\sim}\right)=p\left\{\partial_{x} \mathcal{S}_{\omega} f\right\}^{\sim}$ we obtain, as in the proof of Lemma G.3, that for $s \geq 1$

$$
\left\|\partial_{y}^{\beta} \widetilde{\mathcal{S}_{\omega} f}\right\|_{s} \leq c_{\beta, s}\left(\left\|\partial_{x}^{\beta} \mathcal{S}_{\omega} f\right\|\left\|_{s}\right\| \mathcal{U}_{t}^{-1}\left\|_{C^{1}}^{\beta+s}+\right\| \mathcal{S}_{\omega} f\left\|_{1}\right\| \mathcal{U}_{t}^{-1}\left\|_{C^{\beta+s}}+\right\| \mathcal{S}_{\omega} f \|_{0}\right),
$$


where (see Appendix G)

$$
\left\|\mathcal{U}_{t}^{-1}\right\|_{C^{l}} \leq c_{l}\left(M_{4}\right)\|w\|_{l+3} .
$$

It follows that

$$
\left\|\partial_{y}^{\beta} \widetilde{\mathcal{S}_{\omega} f}\right\|_{s} \leq c_{\beta, s}\left(M_{4}\right)\left(\left\|\partial_{x}^{\beta} \mathcal{S}_{\omega} f\right\|_{s}+\|w\|_{\beta+s+3}\left\|\mathcal{S}_{\omega} f\right\|_{1}+\left\|\mathcal{S}_{\omega} f\right\|_{0}\right)
$$

hence, for $s \geq 1$

$$
\begin{aligned}
\left\|G_{0, \beta} \widetilde{\mathcal{S}_{\omega} f}\right\|_{s} \leq & c_{\beta, s}\left(M_{4}\right)\left(\left\|G_{0, \beta} \mathcal{S}_{\omega} f\right\|_{s}+\|w\|_{\beta+s+3}\left\|\mathcal{S}_{\omega} f\right\|_{1}+\left\|\mathcal{S}_{\omega} f\right\|_{0}\right) \\
\leq & c_{\beta, s}\left(M_{4}\right)\left\{\|w\|_{\beta+s+r+1}\|f\|_{0}+\|w\|_{\beta+r+1}\|f\|_{s}+\right. \\
& \left.+\|w\|_{\beta+s+3}\left(\|w\|_{r+2}\|f\|_{0}+\|w\|_{r+1}\|f\|_{1}\right)+\|w\|_{r+1}\|f\|_{0}\right\} .
\end{aligned}
$$

Since

$$
\begin{aligned}
\|w\|_{\beta+s+3}\|w\|_{r+2} & \leq c_{\beta, s}\|w\|_{\beta+s+r+1}\|w\|_{4} \\
\|w\|_{\beta+s+3}\|w\|_{r+1} & \leq c_{\beta, s}\|w\|_{\beta+r+s}\|w\|_{4} \text { for } r \geq 3, s \geq 1 \\
\|w\|_{\beta+r+s}\|f\|_{1} & \leq c_{\beta, s}\left(\|w\|_{\beta+s+r+1}\|f\|_{0}+\|w\|_{\beta+r+1}\|f\|_{s}\right)
\end{aligned}
$$

we obtain the estimates of Corollary 6.7.

\section{Proof of Lemma 6.8}

By definition,

$$
\mathcal{S} u(y, t)=(\mathcal{H} v)\left(\mathcal{U}_{t}^{-1}(y), t\right)-(\mathcal{H} u)(y, t) \text { where } v(x, t)=u\left(\mathcal{U}_{t}(x), t\right),
$$

where, from (6.14),

$$
\begin{aligned}
(\mathcal{H} v)\left(\mathcal{U}_{t}^{-1}(y), t\right) & =-\frac{p \cdot v \cdot}{2 \pi} \int_{-\pi}^{\pi} \frac{v(z, t) d z}{\tan \frac{1}{2}\left(\mathcal{U}_{t}^{-1}(y)-z\right)} \\
& =-\frac{p \cdot v \cdot}{2 \pi} \int_{-\pi}^{\pi} \frac{u(\zeta, t))\left(1-\partial_{y} \widetilde{d}(\zeta, t)\right) d \zeta}{\tan \frac{1}{2}\left(\mathcal{U}_{t}^{-1}(y)-\mathcal{U}_{t}^{-1}(\zeta)\right)} \\
& =-\frac{p \cdot v \cdot}{2 \pi} \int_{-\pi}^{\pi} \frac{u(\zeta, t)\left(\partial_{y} \mathcal{U}_{t}^{-1}\right)(\zeta) d \zeta}{\tan \frac{1}{2}\left(\mathcal{U}_{t}^{-1}(y)-\mathcal{U}_{t}^{-1}(\zeta)\right)} \\
& =\frac{p \cdot v \cdot}{\pi} \int_{-\pi}^{\pi} u(\zeta, t) \frac{\partial}{\partial \zeta} \log \left|\sin \frac{1}{2}\left(\mathcal{U}_{t}^{-1}(y)-\mathcal{U}_{t}^{-1}(\zeta)\right)\right| d \zeta .
\end{aligned}
$$

Hence

$$
(\mathcal{S} u)(y, t)=\frac{1}{\pi} \int_{-\pi}^{\pi} u(\zeta, t) \frac{\partial}{\partial \zeta} \log \left|\frac{\sin \frac{1}{2}\left(\mathcal{U}_{t}^{-1}(y)-\mathcal{U}_{t}^{-1}(\zeta)\right)}{\sin \frac{1}{2}(y-\zeta)}\right| d \zeta
$$


which, as we will soon see, is no longer a principal-value integral. Since $\mathcal{U}_{t}^{-1}(y)=$ $y-\tilde{d}(y, t)$,

$$
\begin{aligned}
& K(y, \zeta):=\frac{\sin \frac{1}{2}\left(\mathcal{U}_{t}^{-1}(y)-\mathcal{U}_{t}^{-1}(\zeta)\right)}{\sin \frac{1}{2}(y-\zeta)} \\
& =\cos \frac{1}{2}(\tilde{d}(y, t)-\tilde{d}(\zeta, t))-\cot \frac{1}{2}(y-\zeta) \int_{0}^{1} \frac{d}{d s}\left(\sin \frac{1}{2}(\tilde{d}(y, t)-\tilde{d}(y+s(\zeta-y), t))\right) d s \\
& =1+\left(\cos \frac{1}{2}(\tilde{d}(y, t)-\tilde{d}(\zeta, t))-1\right)- \\
& \frac{1}{2}(y-\zeta) \cot \frac{1}{2}(y-\zeta)\left(\int_{0}^{1} \partial_{y} \tilde{d}(y+s(\zeta-y), t) \cos \frac{1}{2}(\tilde{d}(y, t)-\tilde{d}(y+s(\zeta-y), t)) d s\right) .
\end{aligned}
$$

Therefore for $d \in C^{m-3}$,

$(\mathcal{S} u)(y, t)=\frac{1}{\pi} \int_{-\pi}^{\pi} \partial_{\zeta} \log K(\zeta, y, t) u(\zeta, t) d \zeta=-\frac{1}{\pi} \int_{-\pi}^{\pi} \log K(\zeta, y, t) \partial_{\zeta} u(\zeta, t) d \zeta$ where $K \in C^{m-4}$. It follows that $\mathcal{S}(u) \in C^{m-4}$ and, for $u \in H_{\text {叫, }}^{s}$,

$$
\left\|G_{0, \beta} \mathcal{S} u\right\|_{s} \leq c_{\beta, s}\left\{\|\tilde{d}\|_{C^{\beta+s+2}}\|u\|_{0}+\|\tilde{d}\|_{C^{\beta+2}}\|u\|_{s}\right\}, \quad 0 \leq \beta+s \leq m-5 .
$$

An appeal to (G.3) yields the required estimate and the Q1D property of $\mathcal{S}$ follows. This completes the proof of Lemma 6.8. $\quad \square$ For future reference we now observe that the operator $\mathcal{S}$ may be expanded in "powers" of $d$. Note first that

$$
\begin{aligned}
v(x, t)=u\left(\mathcal{U}_{t}(x), t\right) & =u(x, t)+d(x, t) \partial_{x} u(x, t)+\frac{1}{2} d(x, t)^{2} \partial_{x x} u(x, t)+\ldots \\
\mathcal{H} v(x, t) & =\mathcal{H} u(x, t)+\mathcal{H}\left(d \partial_{x} u\right)(x, t)+\frac{1}{2} \mathcal{H}\left(d^{2} \partial_{x x} u\right)(x, t)+. .
\end{aligned}
$$

Since $d(x, t)=\widetilde{d}(x+d(x, t), t)=\widetilde{d}(x, t)+\widetilde{d}(x, t) \partial_{y} \widetilde{d}(x, t)+\ldots$, and, from (6.13),

$$
g\left(\mathcal{U}_{t}^{-1}(x), t\right)=g(x, t)-\widetilde{d}(x, t) \partial_{x} g(x, t)+\widetilde{d}(x, t)^{2} \partial_{x x} g(x, t) / 2+\ldots,
$$

we obtain (with ' denoting differentiation with respect to the first variable) that if $u \in C^{2}$

$$
\mathcal{S} u=\mathcal{S}_{\tilde{d}} u^{\prime}+\mathcal{S}_{\tilde{d}}\left(\tilde{d}^{\prime} u^{\prime}\right)+\frac{1}{2}\left\{\mathcal{S}_{\tilde{d}}\left(\tilde{d} u^{\prime \prime}\right)-\tilde{d} \mathcal{S}_{\tilde{d}} u^{\prime \prime}\right\}+O\left(\|d\|_{\infty}^{3}\right) .
$$

\section{J Proof of Corollary 6.10}

\section{Calculation of $q$ and $p$}

By definition

$$
q(y, t)=\left\{(b-\mu)\left(1+d^{\prime}\right)\right\}\left(\mathcal{U}_{t}^{-1}(y), t\right)
$$


and, from the last part of Lemma 5.1,

$$
\mu-b=1+2 \varepsilon \cos x \cos t-\varepsilon^{2}\left(-\frac{5}{4}+\cos 2 x(1+\cos 2 t)\right)+O\left(\varepsilon^{3}\right),
$$

and $d$ is given by (6.11). Then

$$
\begin{aligned}
(\mu-b)(1 & \left.+d^{\prime}\right) \\
& =1+2 \varepsilon \cos x-\varepsilon^{2}\left(-\frac{1}{4}-2 \cos t+\cos 2 t+2 \cos t \cos 2 x\right)+O\left(\varepsilon^{3}\right) .
\end{aligned}
$$

Now observe that when $y=\mathcal{U}_{t}(x)=1+d(x, t)$

$$
\begin{aligned}
\cos x & =\cos y+\varepsilon(1-\cos t)(1-\cos 2 y)+O\left(\varepsilon^{2}\right), \\
\sin x & =\sin y-\varepsilon(1-\cos t) \sin 2 y+O\left(\varepsilon^{2}\right) .
\end{aligned}
$$

This gives that, as $\varepsilon \rightarrow 0$, in $C_{\text {她 }}^{m-4, e e}$,

$$
q(y, t)=1+2 \varepsilon \cos y-\varepsilon^{2}\left(-\frac{9}{4}+\cos 2 t+2 \cos 2 y\right)+O\left(\varepsilon^{3}\right) .
$$

In the same way (6.11) yields that in $C_{\text {如 }}^{m-3, o e}$,

$$
\tilde{d}(y, t)=2 \varepsilon(1-\cos t) \sin y-2 \varepsilon^{2}(1-\cos t) \sin 2 y+O\left(\varepsilon^{3}\right) .
$$

Finally, in $C_{\text {㛎 }}^{m-4, e e}$,

$$
\begin{aligned}
p(y, t) & =1-\left(\partial_{y} \tilde{d}\right)(y, t) \\
& =1+2 \varepsilon(\cos t-1) \cos y+4 \varepsilon^{2}(1-\cos t) \cos 2 y+O\left(\varepsilon^{3}\right) .
\end{aligned}
$$

\section{Computation of $\mathcal{G}$}

To calculate $-\mathcal{H S}_{a}\left(\widehat{\partial_{t} \varphi / p}\right)$, first note from (5.4) and (6.11) that for $f \in H_{\natural \natural}^{k-1, e e}$, $k \leq m-4$, as $\varepsilon \rightarrow 0$,

$$
\begin{aligned}
\mathcal{S}_{a}(\widehat{f})= & 2 \varepsilon \sin t \mathcal{S}_{\sin x}(\widehat{f})-\varepsilon^{2} \sin 2 t \mathcal{S}_{\sin 2 x} f+O\left(\varepsilon^{3}\|f\|_{k-1}\right) \\
= & 2 \varepsilon \sin t \mathcal{S}_{\sin x}\left(f+d f^{\prime}\right)-\varepsilon^{2} \sin 2 t \mathcal{S}_{\sin 2 x} f+O\left(\varepsilon^{3}\|f\|_{k-1}\right) \\
= & 2 \varepsilon \sin t \mathcal{S}_{\sin x} f+4 \varepsilon^{2} \sin t(1-\cos t) \mathcal{S}_{\sin x}\left(f^{\prime} \sin x\right) \\
& -\varepsilon^{2} \sin 2 t \mathcal{S}_{\sin 2 x} f+O\left(\varepsilon^{3}\|f\|_{k-1}\right) .
\end{aligned}
$$

When $g$ is an even functions of $x$, we have the identities

$$
\begin{aligned}
\mathcal{S}_{\sin x} g & =\pi_{0}(g \cos x)+(\cos x) \pi_{0} g, \\
\mathcal{S}_{\sin 2 x} g & =\pi_{0}(g \cos 2 x)+2(\cos x) \pi_{0}(g \cos x)+(\cos 2 x) \pi_{0} g,
\end{aligned}
$$

which gives

$$
\mathcal{S}_{\sin x}\left(g^{\prime} \sin x\right)=-\pi_{0}(g \cos 2 x)-(\cos x) \pi_{0}(g \cos x) .
$$


Therefore, if $f$ is an even function of $y$,

$$
\begin{aligned}
\mathcal{S}_{a}(\widehat{f})= & 2 \varepsilon \sin t\left\{\pi_{0}(f \cos y)+(\cos x) \pi_{0} f\right\}+ \\
& -4 \varepsilon^{2} \sin t\left\{\pi_{0}(f \cos 2 y)+(\cos x) \pi_{0}(f \cos y)\right\}+ \\
& +\varepsilon^{2} \sin 2 t\left\{\pi_{0}(f \cos 2 y)-(\cos 2 x) \pi_{0} f\right\}+O\left(\varepsilon^{3}\|f\|_{k-1}\right),
\end{aligned}
$$

and

$$
\begin{aligned}
-\mathcal{H S}_{a}(\widehat{f})= & 2 \varepsilon(\sin t \sin x) \pi_{0} f-4 \varepsilon^{2}(\sin t \sin x) \pi_{0}(f \cos y)+ \\
& -\varepsilon^{2}(\sin 2 t \sin 2 x) \pi_{0} f+O\left(\varepsilon^{3}\|f\|_{k-1}\right)
\end{aligned}
$$

Now we need to replace $f$ by $\partial_{t} \varphi / p, \varphi \in H_{\text {ŁŁ }}^{k, e e}, k \leq m-4$. From (J.3),

$$
\partial_{t} \varphi / p=\partial_{t} \varphi\left(1+2 \varepsilon(1-\cos t) \cos y+O\left(\varepsilon^{2}\|\varphi\|_{k}\right)\right.
$$

and so

$$
\begin{aligned}
& -\mathcal{H S}_{a}\left(\widehat{\partial_{t} \varphi / p}\right)=2 \varepsilon(\sin t \sin x) \pi_{0}\left(\partial_{t} \varphi\right) \\
& \quad-2 \varepsilon^{2}(\sin 2 t \sin x) \pi_{0}\left(\partial_{t} \varphi \cos y\right) \\
& \quad-\varepsilon^{2}(\sin 2 t \sin 2 x) \pi_{0}\left(\partial_{t} \varphi\right)+O\left(\varepsilon^{3}\|\varphi\|_{k}\right),
\end{aligned}
$$

which gives

$$
\begin{aligned}
& \left\{-\mathcal{H} \mathcal{S}_{a}\left(\widehat{\partial_{t} \varphi / p}\right)\right\}^{\sim}(y, t) \\
& =2 \varepsilon(\sin t \sin y) \pi_{0}\left(\partial_{t} \varphi\right)-2 \varepsilon^{2}(\sin t \sin 2 y) \pi_{0}\left(\partial_{t} \varphi\right) \\
& \quad-2 \varepsilon^{2}(\sin 2 t \sin y) \pi_{0}\left(\partial_{t} \varphi \cos y\right)+O\left(\varepsilon^{3}\|\varphi\|_{k}\right),
\end{aligned}
$$

whence

$$
\begin{aligned}
&-\partial_{y}\left(\left\{\mathcal{H} \mathcal{S}_{a}(\widehat{\partial \varphi / p})\right\}^{\sim}\right)(y, t) \\
&=2 \varepsilon(\sin t \cos y) \pi_{0}\left(\partial_{t} \varphi\right)-4 \varepsilon^{2}(\sin t \cos 2 y) \pi_{0}\left(\partial_{t} \varphi\right) \\
&-2 \varepsilon^{2}(\sin 2 t \cos y) \pi_{0}\left(\partial_{t} \varphi \cos y\right)+O\left(\varepsilon^{3}\|\varphi\|_{k}\right) .
\end{aligned}
$$

Now to calculate $-\partial_{y}\left(\mathcal{S}_{q} \varphi\right)$ note from (J.1) that

$$
\mathcal{S}_{q} \varphi=2 \varepsilon \mathcal{S}_{\cos y} \varphi-2 \varepsilon^{2} \mathcal{S}_{\cos 2 y} \varphi+O\left(\varepsilon^{3}\|\varphi\|_{k}\right),
$$

and that, for an even function $f$,

$$
\begin{aligned}
\mathcal{S}_{\cos y} f & =-(\sin y) \pi_{0} f, \\
\mathcal{S}_{\cos 2 y} f & =-2(\sin y) \pi_{0}(f \cos y)-(\sin 2 y) \pi_{0} f .
\end{aligned}
$$

Therefore

$$
\begin{aligned}
\mathcal{S}_{q} \varphi=-2 \varepsilon(\sin y) \pi_{0} \varphi & \\
& +4 \varepsilon^{2}(\sin y) \pi_{0}(\varphi \cos y)+2 \varepsilon^{2}(\sin 2 y) \pi_{0} \varphi+O\left(\varepsilon^{3}\|\varphi\|_{k}\right),
\end{aligned}
$$


and

$$
\begin{aligned}
-\partial_{y}\left(\mathcal{S}_{q} \varphi\right)= & 2 \varepsilon(\cos y) \pi_{0} \varphi \\
& -4 \varepsilon^{2}(\cos y) \pi_{0}(\varphi \cos y)-4 \varepsilon^{2}(\cos 2 y) \pi_{0} \varphi+O\left(\varepsilon^{3}\|\varphi\|_{k}\right) .
\end{aligned}
$$

Now we need to compute $\mathcal{S} u$ up to order $\varepsilon^{2}$. Recall from (I.2) and (J.2) that

$$
\begin{aligned}
\mathcal{S} f & =\mathcal{S}_{\tilde{d}} f^{\prime}+\mathcal{S}_{\tilde{d}}\left(\tilde{d}^{\prime} f^{\prime}\right)+\frac{1}{2}\left\{\mathcal{S}_{\tilde{d}}\left(\tilde{d} f^{\prime \prime}\right)-\tilde{d} \mathcal{S}_{\tilde{d}} f^{\prime \prime}\right\}+O\left(\|d\|^{3}\right) \\
\widetilde{d}(y, t) & =2 \varepsilon(1-\cos t) \sin y-2 \varepsilon^{2}(1-\cos t) \sin 2 y+O\left(\varepsilon^{3}\right)
\end{aligned}
$$

and note the following identities for even functions $f$ of $y$ :

$$
\mathcal{S}_{\sin y}\left(f^{\prime}\right)=\left(\mathcal{S}_{\sin y} f\right)^{\prime}-\mathcal{S}_{\cos y} f=0,
$$

by (J.5) and (J.9), and hence

$$
\mathcal{S}_{\sin y}\left(f^{\prime} \cos y\right)=0=\mathcal{S}_{\sin y}\left(f^{\prime \prime} \sin y\right) .
$$

Also

$$
\mathcal{S}_{\sin 2 y}\left(f^{\prime}\right)=\left(\mathcal{S}_{\sin 2 y} f\right)^{\prime}-2 \mathcal{S}_{\cos 2 y} f=2(\sin y) \pi_{0}(f \cos y),
$$

by (J.6) and (J.10) and

$$
\mathcal{S}_{\sin y}\left(f^{\prime \prime}\right)=-\pi_{0}(f \cos y) \text { by (J.5). }
$$

This leads to

$$
\begin{aligned}
\mathcal{S} \varphi & =-2 \varepsilon^{2}(1-\cos t) \mathcal{S}_{\sin 2 y} \varphi^{\prime}-2 \varepsilon^{2}(1-\cos t)^{2} \sin y \mathcal{S}_{\sin y}\left(\varphi^{\prime \prime}\right)+O\left(\varepsilon^{3}\|\varphi\|_{k}\right), \\
& =\varepsilon^{2} \sin y(\cos 2 t-1) \pi_{0}(\varphi \cos y)+O\left(\varepsilon^{3}\|\varphi\|_{k}\right) .
\end{aligned}
$$

From (J.1) we conclude that

$$
\mathcal{S}(q \varphi)=\mathcal{S} \varphi+O\left(\varepsilon^{3}\|\varphi\|_{k}\right)
$$

and

$$
\begin{aligned}
-\partial_{y} \mathcal{S}(q \varphi) & =-\partial_{y} \mathcal{S} \varphi+O\left(\varepsilon^{3}\|\varphi\|_{k}\right) \\
& =-\varepsilon^{2} \cos y(\cos 2 t-1) \pi_{0}(\varphi \cos y)+O\left(\varepsilon^{3}\|\varphi\|_{k}\right) .
\end{aligned}
$$

Collecting (J.8), (J.11), (J.12) gives the leading terms of $\mathcal{G}$, which completes the proof. 


\section{K Proof of Lemma 7.1}

Before establishing the Q1D properties of $\mathcal{S}_{(0)}$ we give a refinement of [22, Prop 7.8] on Q1D properties of integral operators of the form

$$
A u(\xi, \tau)=\int_{-\pi}^{\pi} \int_{0}^{1} a(\xi, \tau, r, \zeta) u(\zeta, \tau+r \omega(\xi, \tau, \zeta)) d r d \zeta,
$$

in which the kernels $a(\xi, \tau, r, \zeta)$ and the function $\omega(\xi, \tau, \zeta)$ are $2 \pi$-periodic with respect to $\xi, \tau$ and $\zeta$.

Proposition K.1. For some $l \in \mathbb{N}$ suppose that $a \in C^{l}$ and $\|\omega\|_{C^{1}} \leq M$, where $1+D_{1} \omega>c_{1}^{-1}$ for some $c_{1}>0$. Then, for $0 \leq s \leq l$,

$$
\|A u\|_{s} \leq c_{s}\left(M, c_{1}\right)\left(\|a\|_{C^{0}}\left(\|u\|_{s}+\|u\|_{1}\|\omega\|_{C^{s}}\right)+\|a\|_{C^{s}}\|u\|_{0}\right),
$$

and, for $0 \leq \beta+s \leq l$,

$$
\left\|G_{\beta, \beta} A u\right\|_{s} \leq c_{s}\left(M, c_{1}\right)\left\{\|a\|_{C^{\beta}}\left(\|u\|_{s}+\|u\|_{1}\|\omega\|_{C^{s}}\right)+\|a\|_{C^{\beta+s}}\|u\|_{0}\right\},
$$

Proof. Denote $u(\zeta, \tau+r \omega(\xi, \tau, \zeta))$ by $v(\xi, \tau, r, \zeta)$. For convenience, let $D_{1}=$ $\partial / \partial \tau$ and $D_{2}=\partial / \partial_{\xi}$. Then, for $i=1,2$,

$$
\begin{aligned}
& \left|\left(D_{i}\right)^{k}(A u)(\xi, \tau)\right|^{2}=\left|\int_{-\pi}^{\pi} \int_{0}^{1}\left(D_{i}\right)^{k}(a(\xi, \tau, r, \zeta) v(\xi, \tau, r, \zeta)) d r d \zeta\right|^{2} \\
& =\left|\int_{-\pi}^{\pi} \int_{0}^{1} \sum_{j=0}^{k}\left(\begin{array}{c}
j \\
k
\end{array}\right)\left(\left(D_{i}\right)^{j} a(\xi, \tau, r, \zeta)\right)\left(D_{i}\right)^{k-j}(v(\xi, \tau, r, \zeta)) d r d \zeta\right|^{2} \\
& \leq c(k) \int_{-\pi}^{\pi} \int_{0}^{1} \sum_{j=0}^{k}\|a\|_{C^{j}}^{2} \mid\left(D_{i}\right)^{k-j}\left(\left.v(\xi, \tau, r, \zeta)\right|^{2} d r d \zeta .\right.
\end{aligned}
$$

Let

$$
\|\mid\| v\left\|_{s}^{2}=\int_{-\pi}^{\pi} \int_{0}^{1}\right\| v(\cdot, \cdot, r, \zeta) \|_{s}^{2} d r d \zeta .
$$

Then, by the interpolation inequalities in the periodic-function spaces $C^{s}$ and $H^{s}$ and Hölder's inequality,

$$
\begin{aligned}
& \int_{-\pi}^{\pi} \int_{-\pi}^{\pi}\left|\left(D_{i}\right)^{k}(A u)(\xi, \tau)\right|^{2} d \xi d \tau \leq c(k)\left(\sum_{j=0}^{k}\|a\|_{C^{j}} \mid\|v\| \|_{k-j}\right)^{2} \\
& \quad \leq c(k)\left(\sum_{j=0}^{k}\|a\|_{C^{k}}^{j / k}\|a\|_{C^{0}}^{1-j / k} \mid\|v\|\left\|_{k}^{1-j / k}\right\| v \|_{0}^{j / k}\right)^{2} \\
& \quad \leq c(k)\left\{\|a\|_{C^{0}}\left|\|v\|_{k}+\|a\|_{C^{k}}\|v \mid\|_{0}\right\}^{2}\right.
\end{aligned}
$$


Since $\omega \in C^{l}$ and $\|\omega\|_{C^{1}} \leq M$, it follows from [18, Theorem 3.1.5] that $v \in H^{j}$ when $u \in H^{j}$ for $0 \leq j \leq l$ and, by Lemmas G.1 and G.2,

$$
\begin{aligned}
\|\| v \|_{j} & \leq c_{j}\left(\|u\|_{j}\left(1+\|\omega\|_{C^{1}}^{j}\right)+\|u\|_{1}\left(1+\|\omega\|_{C^{j}}\right)+\|u\|_{0}\right) \\
& \leq c_{j}\left(M, c_{1}\right)\left(\|u\|_{j}+\|u\|_{1}\|\omega\|_{C^{j}}\right) .
\end{aligned}
$$

Hence

$$
\begin{aligned}
& \int_{-\pi}^{\pi} \int_{-\pi}^{\pi}\left|\left(D_{i}\right)^{k}(A u)(\xi, \tau)\right|^{2} d \xi d \tau \\
& \left.\quad \leq c_{k}\left(M, c_{1}\right)\left\{\|a\|_{C^{0}}\left(\|u\|_{k}+\|u\|_{1}\|\omega\|_{C^{k}}\right)+\|a\|_{C^{k}}\|u\|_{0}\right)\right\}^{2} .
\end{aligned}
$$

Similar analysis for the mixed derivatives leads to (K.1). To prove (K.2) we use induction on $\beta, 0 \leq \beta \leq l-s$. By (K.1) the result holds with $\beta=0$. Assume that it holds for some $0 \leq \beta \leq l-s-1$. It easy to check that $D_{2} A u=D_{1} A_{1} u+A_{2} u$, where the integral operators $A_{i}$ are given by

$$
A_{i} u(y)=\int_{-\pi}^{\pi} \int_{0}^{1} a_{i}(\xi, \tau, r, \zeta) u(\zeta, \tau+r \omega(\xi, \tau, \zeta)) d r d \zeta
$$

with the kernels $a_{1}(\xi, \tau, r, \zeta)=a(\xi, \tau, r, \zeta) \chi(\xi, \tau, r, \zeta)$ and

$$
a_{2}(y, s, \tau)=D_{2} a(\xi, \tau, r, \zeta)-D_{1} a_{1}(\xi, \tau, r, \zeta),
$$

where $\chi=r D_{2} \omega\left(1+r D_{1} \omega\right)^{-1}$. Now, from the triangle inequality and the identity $D_{2} A=D_{1} A_{1}+A_{2}$,

$$
\begin{aligned}
\left\|G_{\beta+1, \beta+1} A u\right\|_{s} & \leq c\left(\left\|G_{\beta, \beta}\left(I+\left|D_{1}\right|\right)^{-1} A u\right\|_{s}+\left\|G_{\beta, \beta}\left(I+\left|D_{1}\right|\right)^{-1} D_{2} A u\right\|_{s}\right. \\
& \leq c\left(\left\|G_{\beta, \beta} A u\right\|_{s}+\left\|G_{\beta, \beta} A_{1} u\right\|_{s}+\left\|G_{\beta, \beta} A_{2} u\right\|_{s}\right) .
\end{aligned}
$$

Now $a \in C^{l}, \omega \in C^{l+1}$ implies $a_{i} \in C^{l-1}, i=1,2$ and therefore, by the inductive hypothesis,

$$
\begin{aligned}
\left\|G_{\beta, \beta} A_{i} u\right\|_{s} & \leq c\left(s, M, c_{1}\right)\left\{\left\|a_{i}\right\|_{C^{\beta}}\left(\|u\|_{s}+\|u\|_{1}\|\omega\|_{C^{s}}\right)+\left\|a_{i}\right\|_{C^{\beta+s}}\|u\|_{0}\right\} \\
& \leq c\left(s, M, c_{1}\right)\left\{\|a\|_{C^{\beta+1}}\left(\|u\|_{s}+\|u\|_{1}\|\omega\|_{C^{s}}\right)+\|a\|_{C^{\beta+1+s}}\|u\|_{0}\right\}
\end{aligned}
$$

for $0 \leq \beta+s \leq l$. Result (K.2) follows by induction.

Now to establish the Q1D properties of $\mathcal{S}_{(0)}$ define functions $\varrho$ and $\ell$ by

$$
Q^{-1}(\xi, \tau)=(\varrho(\xi), \tau-\ell(\xi, \tau))
$$

and observe that

$$
\{\mathcal{H}(\theta \circ Q)\}(y, t)=-\frac{p \cdot v \cdot}{2 \pi} \int_{-\pi}^{\pi} \frac{\theta\left(s+d_{0}(s), t+e_{0}(s, t)\right) d s}{\tan \frac{1}{2}(y-s)} .
$$


Hence, with $t=\tau-\ell(\xi, \tau)$,

$$
\begin{aligned}
& \{\mathcal{H}(\theta \circ Q)\} Q^{-1}(\xi, \tau)=-\frac{p \cdot v \cdot}{2 \pi} \int_{-\pi}^{\pi} \frac{\theta\left(s+d_{0}(s), t+e_{0}(s, t)\right) d s}{\tan \frac{1}{2}(\varrho(\xi)-s)} \\
& =-\frac{p \cdot v \cdot}{2 \pi} \int_{-\pi}^{\pi} \frac{\left.\varrho^{\prime}(\zeta)\right) \theta\left(\zeta, t+e_{0}(\varrho(\zeta), t)\right) d \zeta}{\tan \frac{1}{2}(\varrho(\xi)-\varrho(\zeta))} \\
& =\frac{p \cdot v \cdot}{\pi} \int_{-\pi}^{\pi} \theta\left(\zeta, t+e_{0}(\varrho(\zeta), t)\right) \frac{\partial}{\partial \zeta} \log \left|\sin \frac{1}{2}(\varrho(\xi)-\varrho(\zeta))\right| d \zeta .
\end{aligned}
$$

Now $t=\tau-\ell(\xi, \tau)$ implies that $\ell(\xi, \tau)=e_{0}(\varrho(\xi), t)$ and so

$$
\begin{array}{r}
\theta\left(\zeta, t+e_{0}(\varrho(\zeta), t)\right)=\theta\left(\zeta, \tau-\ell(\xi, \tau)+e_{0}(\varrho(\zeta), \tau-\ell(\xi, \tau))\right) \\
=\theta(\zeta, \tau)+\int_{0}^{1} \frac{\partial}{\partial r} \theta\left(\zeta, \tau-r\left(\ell(\xi, \tau)-e_{0}(\varrho(\zeta), \tau-\ell(\xi, \tau))\right) d r\right. \\
=\theta(\zeta, \tau)+\int_{0}^{1} \frac{\partial}{\partial r} \theta\left(\zeta, \tau+r\left(e_{0}(\varrho(\zeta), t)-e_{0}(\varrho(\xi), t)\right) d r\right. \\
=\theta(\zeta, \tau)+\left(e_{0}(\varrho(\zeta), t)-e_{0}(\varrho(\xi), t)\right) \times \\
\int_{0}^{1} \partial_{\tau} \theta\left(\zeta, \tau+r\left(e_{0}(\varrho(\zeta), t)-e_{0}(\varrho(\xi), t)\right) d r\right.
\end{array}
$$

where

$$
\begin{aligned}
e_{0}(\varrho(\zeta), t)-e_{0}(\varrho(\xi), t) & =\int_{0}^{1} \frac{\partial}{\partial \rho} e_{0}(\varrho(\xi+\rho(\zeta-\xi)), t) d \rho \\
& =(\zeta-\xi) \int_{0}^{1} \varrho^{\prime}(\xi+\rho(\zeta-\xi)) \partial_{y} e_{0}(\varrho(\xi+\rho(\zeta-\xi)), t) d \rho .
\end{aligned}
$$

Therefore

$$
\begin{aligned}
\mathcal{S}_{(0)} \theta(\xi, \tau)= & \int_{-\pi}^{\pi} K_{1}(\xi, \zeta) \theta(\zeta, \tau) d \zeta \\
& +\int_{-\pi}^{\pi} \int_{0}^{1} K_{2}(\xi, \tau, \zeta) \partial_{\tau} \theta\left(\zeta, \tau+r\left(e_{0}(\varrho(\zeta), t)-e_{0}(\varrho(\xi), t)\right) d r d \zeta\right.
\end{aligned}
$$

$=I_{1} \theta(\xi, \tau)+I_{2} \partial_{\tau} \theta(\xi, \tau)$, say, where

$$
\begin{aligned}
K_{1}(\xi, \zeta) & =\frac{1}{\pi} \frac{\partial}{\partial \zeta} \log \left|\frac{\sin \frac{1}{2}(\varrho(\xi)-\varrho(\zeta))}{\sin \frac{1}{2}(\xi-\zeta)}\right| \\
K_{2}(\xi, \tau, \zeta) & =-\frac{1}{2 \pi}(\zeta-\xi) \varrho^{\prime}(\zeta) \cot \frac{1}{2}(\varrho(\xi)-\varrho(\zeta)) \\
& \times \int_{0}^{1} \varrho^{\prime}(\xi+\rho(\zeta-\xi)) \partial_{y} e_{0}(\varrho(\xi+\rho(\zeta-\xi)), t) d \rho
\end{aligned}
$$


Since $d_{0}$, and hence $\varrho$, is in $C^{m-3}$ it follows, as for $K$ in (I.1), that $K_{1}$ is in $C^{m-5}$. Similarly, since $e_{0}$ is in $C^{m-4}$ it follows that $K_{2}$ is in $C^{m-5}$. Now note that

$$
I_{2}\left(\partial_{\tau} \theta\right)=\partial_{\tau}\left(J_{1} \theta\right)+J_{2} \theta
$$

where

$$
\begin{gathered}
J_{1} \theta=\int_{-\pi}^{\pi} \int_{0}^{1} K_{3}(\xi, \tau, \zeta) \theta\left(\zeta, \tau+r\left(e_{0}(\varrho(\zeta), t)-e_{0}(\varrho(\xi), t)\right)\right) d r d \zeta \\
J_{2} \theta=-\int_{-\pi}^{\pi} \int_{0}^{1}\left(\partial_{\tau} K_{3}\right)(\xi, \tau, \zeta) \theta\left(\zeta, \tau+r\left(e_{0}(\varrho(\zeta), t)-e_{0}(\varrho(\xi), t)\right)\right) d r d \zeta
\end{gathered}
$$

and, with $t=\tau-\ell(\xi, \tau)$,

$$
K_{3}(\xi, \tau, \zeta)=\frac{K_{2}(\xi, \tau, \zeta)}{1+r\left(\partial_{t} e_{0}(\varrho(\zeta), t)-\partial_{t} e_{0}(\varrho(\xi), t)\right)\left(1-\partial_{\tau} \ell(\xi, \tau)\right)}
$$

It follows that $K_{3} \in C^{m-5}, \partial_{\tau} K_{3} \in C^{m-6}$ and

$\left\|K_{1}\right\|_{C^{s-5}}+\left\|K_{2}\right\|_{C^{s-5}}+\left\|K_{3}\right\|_{C^{s-5}}+\left\|\partial_{\tau} K_{3}\right\|_{C^{s-6}} \leq c_{m}\left(M_{6}\right)\|w\|_{s}, \quad 5 \leq s \leq m$.

Note that $I_{1}, J_{1}$ and $J_{2}$ are examples from the class of operators $A$ in Proposition $\mathrm{K} .1$ where $\omega=0$ for $I_{1}$ and, for $J_{1}$ and $J_{2}, \omega \in C^{m-4}$ is given by

$$
\omega(\xi, \tau, \zeta)=e_{0}(\varrho(\zeta), \tau-\ell(\xi, \tau))-e_{0}(\varrho(\xi), \tau-\ell(\xi, \tau)) .
$$

Hence $\|\omega\|_{C^{m-4}} \leq c_{m}\left(M_{4}\right)\|w\|_{m}$ and the kernel $a$ of $A$ is given by $K_{1}, K_{3}$ or $\partial_{\tau} K_{3}$. For $I_{1}$ and $J_{1}$ let $l=m-5$, for $J_{2}$ let $l=m-6$ and appeal to Proposition $\mathrm{K} .1$ and interpolation inequalities

$$
\begin{aligned}
\|w\|_{\beta+6}\|w\|_{s+4}\|\theta\|_{1} & \leq c_{\beta, s}\|w\|_{5}\left(\|w\|_{\beta+s+6}\|\theta\|_{0}+\|w\|_{\beta+6}\|\theta\|_{s}\right) \\
\|w\|_{s+5}\|\theta\|_{1} & \leq c_{s}\left(\|w\|_{5}\|\theta\|_{s+1}+\|w\|_{s+6}\|\theta\|_{0}\right)
\end{aligned}
$$

to complete the proof.

\section{Estimates of $\mathcal{G}_{0}$ in Lemma 7.2}

In this Appendix we derive the estimates for $\mathcal{G}_{0}(\theta)$ defined in Lemma 7.2. First consider the term

$$
\mathcal{G}_{0}^{(1)}(\theta)=-\left(1+\beta^{(0)}\right)\left\{\partial_{\xi} \mathcal{S}_{(0)} \theta+\delta_{0} \partial_{\tau} \mathcal{S}_{(0)} \theta\right\}+\alpha \mathcal{S}_{(0)} \theta
$$

From Lemma 7.1, estimate (6.11) for $\beta^{(0)}$, and (see Remark 7.3) the fact that

$$
\|\alpha\|_{C^{l}}+\left\|\delta_{0}\right\|_{C^{l}} \leq c_{l}\left(M_{4}\right)\|w\|_{l+5}
$$


we obtain, after interpolation, the inequality

$$
\left\|G_{\beta+2, \beta} \mathcal{G}_{0}^{(1)} \theta\right\|_{s} \leq c_{\beta, s}\left(M_{6}\right)\left\{\|w\|_{\beta+7}\|\theta\|_{s}+\|w\|_{\beta+s+7}\|\theta\|_{0}\right\} .
$$

It remains to estimate $\left\{\left(1+\dot{e}_{0}\right)^{-2} \mathcal{G}(\theta \circ Q)\right\} \circ Q^{-1}$, where, in the notation of Appendix K,

$$
\begin{aligned}
(y, t) & =Q^{-1}(\xi, \tau)=(\varrho(\xi), \tau-\ell(\xi, \tau)), \\
(\xi, \tau) & =Q(y, t)=\left(y+d_{0}(y), t+e_{0}(y, t)\right) .
\end{aligned}
$$

We assume only that $\mathcal{G}$ is a Q1D operator of order $(1, \beta)$, not necessarily the one given in Theorem 6.9. Lemmas G.1 and G.3 with (7.10) lead, after standard interpolation, to

$$
\begin{aligned}
\left\|\left(1+\dot{e}_{0}\right)^{-2} \circ Q^{-1}\right\|_{C^{l}} & \leq c_{l}\left(M_{5}\right)\left(1+\|w\|_{l+4}\right), \\
\|\theta \circ Q\|_{s} & \leq c_{s}\left(M_{5}\right)\left(\|\theta\|_{s}+\|w\|_{s+5}\|\theta\|_{0}\right) .
\end{aligned}
$$

Now we need an estimate of $\mathcal{G}(\varphi) \circ Q^{-1}$ using the estimate of Theorem 6.9 for $\mathcal{G}$. With $\ell_{\tau}=\partial_{\tau} \ell$

$$
\begin{aligned}
\partial_{\tau}\left\{\partial_{t}^{-1}\left(\mathcal{G}(\varphi)\left(1+\dot{e}_{0}\right)\right) \circ Q^{-1}\right\} & =\left(1-\ell_{\tau}\right)\left(\mathbb{I}-\pi_{1}\right)\left(\mathcal{G}(\varphi)\left(1+\dot{e}_{0}\right)\right) \circ Q^{-1} \\
& =\mathcal{G}(\varphi) \circ Q^{-1}-\left(1-\ell_{\tau}\right) \pi_{1}\left(\mathcal{G}(\varphi)\left(1+\dot{e}_{0}\right)\right) \circ \varrho
\end{aligned}
$$

since $\left(1-\ell_{\tau}\right)\left(1+\dot{e}_{0}\right) \circ Q^{-1}=1$. Since $\ell$ is odd in $\tau$,

$$
\partial_{\tau}^{-1}\left(1-\ell_{\tau}\right)=-\partial_{\tau}^{-1} \ell_{\tau}=-\ell,
$$

and it follows that

$$
\begin{aligned}
\partial_{\tau}^{-1}\left(\mathcal{G}(\varphi) \circ Q^{-1}\right)=\left(\mathbb{I}-\pi_{1}\right)\left(\left\{\partial_{t}^{-1}\left(\mathcal{G}(\varphi)\left(1+\dot{e}_{0}\right)\right)\right\} \circ Q^{-1}\right) \\
-\ell \pi_{1}\left(\mathcal{G}(\varphi)\left(1+\dot{e}_{0}\right)\right) \circ \varrho .
\end{aligned}
$$

A change of variables in the integral gives that

$$
\pi_{1}\left(\mathcal{G}(\varphi) \circ Q^{-1}\right)=\pi_{1}\left(\mathcal{G}(\varphi)\left(1+\dot{e}_{0}\right)\right) \circ \varrho .
$$

Now we are in a position to estimate $G_{1,0}\left(\mathcal{G}(\varphi) \circ Q^{-1}\right)$ in terms of $G_{1,0} \mathcal{G}(\varphi)$. First note the obvious inequality (here $c_{s}$ and $c_{s}^{\prime}$ are constants)

$$
\left\|\partial_{\tau}^{-1} u\right\|_{s}+\left\|\pi_{1} u\right\|_{s} \leq c_{s}\left\|G_{1,0} u\right\|_{s} \leq c_{s}^{\prime}\left(\left\|\partial_{\tau}^{-1} u\right\|_{s}+\left\|\pi_{1} u\right\|_{s}\right),
$$

which follows from the discussion of Q1D operators preceding (7.15). Next

$$
\begin{aligned}
\partial_{t}^{-1}\left(\mathcal{G}(\varphi)\left(1+\dot{e}_{0}\right)\right)=(\mathbb{I} & \left.-\pi_{1}\right)\left(1+\dot{e}_{0}\right) \partial_{t}^{-1} \mathcal{G}(\varphi)+e_{0} \pi_{1}(\mathcal{G}(\varphi)) \\
& -\partial_{t}^{-1}\left(\ddot{e}_{0} \partial_{t}^{-1} \mathcal{G}(\varphi)\right)
\end{aligned}
$$

which follows from Lemma M.2 and the fact that $\pi_{1} \dot{e}_{0}=0$, and

$$
\pi_{1}\left(\mathcal{G}(\varphi)\left(1+\dot{e}_{0}\right)\right)=\pi_{1}(\mathcal{G}(\varphi))-\pi_{1}\left(\ddot{e}_{0} \partial_{t}^{-1} \mathcal{G}(\varphi)\right),
$$


which follows from integration by parts since $\mathcal{G}(\varphi)=\pi_{1}(\mathcal{G}(\varphi))+\partial_{t} \partial_{t}^{-1}(\mathcal{G}(\varphi))$. Now it follows that

$$
\left\|G_{1,0}\left(\mathcal{G}(\varphi)\left(1+\dot{e}_{0}\right)\right)\right\|_{s} \leq c_{s}\left(M_{5}\right)\left(\left\|G_{1,0} \mathcal{G}(\varphi)\right\|_{s}+\|w\|_{s+5}\left\|G_{1,0} \mathcal{G}(\varphi)\right\|_{0}\right)
$$

and, from Lemma G.3 and standard interpolation, (for $s \geq 1$ )

$$
\begin{aligned}
\left\|G_{1,0}\left(\mathcal{G}(\varphi) \circ Q^{-1}\right)\right\|_{s} & \leq c_{s}\left(M_{5}\right)\left(\left\|G_{1,0} \mathcal{G}(\varphi)\right\|_{s}+\|w\|_{s+5} \| G_{1,0}\left(\mathcal{G}(\varphi) \|_{0}\right)\right. \\
& \leq c_{s}\left(M_{5}\right)\left(\|w\|_{6}\|\varphi\|_{s}+\|w\|_{s+6}\|\varphi\|_{0}\right),
\end{aligned}
$$

by Theorem 6.9 .

Lemma L.1. If $\mathcal{G}$ is a $Q 1 D$ operator on $H_{\text {uh }}^{s}$ of order $(1, \beta)$ with

$$
\left\|G_{1, \beta} \mathcal{G}(\varphi)\right\|_{s} \leq c_{\beta, s}\left(M_{5}\right)\left(\|w\|_{\beta+6}\|\varphi\|_{s}+\|w\|_{s+\beta+6}\|\varphi\|_{0}\right),
$$

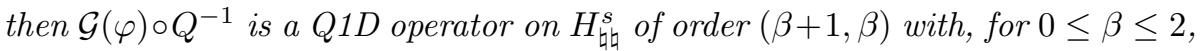

$$
\left\|G_{\beta+1, \beta}\left(\mathcal{G}(\varphi) \circ Q^{-1}\right)\right\|_{s} \leq c_{\beta, s}\left(M_{5}\right)\left(\|w\|_{\beta+6}\|\varphi\|_{s}+\|w\|_{s+\beta+6}\|\varphi\|_{0}\right),
$$

and, for $2 \leq \beta$,

$$
\left\|G_{\beta+1, \beta}\left(\mathcal{G}(\varphi) \circ Q^{-1}\right)\right\|_{s} \leq c_{\beta, s}\left(M_{5}\right)\left(\|w\|_{2 \beta+4}\|\varphi\|_{s}+\|w\|_{s+2 \beta+4}\|\varphi\|_{0}\right) .
$$

Proof. The lemma is already proved for $\beta=0$, see (L.8). Let $\varrho^{\prime}=d \varrho / d \xi$. A differentiation of (L.4) gives

$$
\begin{aligned}
\partial_{\xi} \partial_{\tau}^{-1}\left(\mathcal{G}(\varphi) \circ Q^{-1}\right)= & \varrho^{\prime}\left(\mathbb{I}-\pi_{1}\right)\left(\left\{\partial_{y} \partial_{t}^{-1}\left(\mathcal{G}(\varphi)\left(1+\dot{e}_{0}\right)\right)\right\} \circ Q^{-1}\right) \\
& -\ell_{\xi}\left(\mathcal{G}(\varphi)\left(1+\dot{e}_{0}\right)\right) \circ Q^{-1}-\varrho^{\prime} \ell \pi_{1}\left[\partial_{y}\left(\mathcal{G}(\varphi)\left(1+\dot{e}_{0}\right)\right)\right] \circ \varrho \\
& \left.+\pi_{1}\left\{\ell_{\xi}\left(\mathbb{I}-\pi_{1}\right)\right)\left(\mathcal{G}(\varphi)\left(1+\dot{e}_{0}\right)\right) \circ Q^{-1}\right\}
\end{aligned}
$$

and, since $\pi_{1}\left\{\ell_{\xi}\left(\pi_{1}\left(\mathcal{G}(\varphi)\left(1+\dot{e}_{0}\right)\right) \circ Q^{-1}\right)\right\}=0$ because $\ell_{\xi}$ is odd in $\tau$,

$$
\begin{aligned}
\partial_{\xi} \partial_{\tau}^{-1}\left(\mathcal{G}(\varphi) \circ Q^{-1}\right)= & \varrho^{\prime}\left(\mathbb{I}-\pi_{1}\right)\left(\left\{\partial_{y} \partial_{t}^{-1}\left(\mathcal{G}(\varphi)\left(1+\dot{e}_{0}\right)\right)\right\} \circ Q^{-1}\right) \\
& -\left(\mathbb{I}-\pi_{1}\right)\left\{\ell_{\xi}\left(\mathcal{G}(\varphi)\left(1+\dot{e}_{0}\right)\right) \circ Q^{-1}\right\} \\
& -\varrho^{\prime} \ell \pi_{1}\left[\partial_{y}\left(\mathcal{G}(\varphi)\left(1+\dot{e}_{0}\right)\right)\right] \circ \varrho .
\end{aligned}
$$

Also, from (L.5) and (L.6), by induction and standard interpolation,

$$
\begin{aligned}
& \left\|G_{1, \beta}\left(\mathcal{G}(\varphi)\left(1+\dot{e}_{0}\right)\right)\right\|_{s} \leq c_{\beta, s}\left(M_{5}\right)\left(\|w\|_{\beta+s+5}\left\|G_{1,0} \mathcal{G}(\varphi)\right\|_{0}\right. \\
& \left.\quad+\|w\|_{\beta+5}\left\|G_{1,0} \mathcal{G}(\varphi)\right\|_{s}+\|w\|_{s+5}\left\|G_{1, \beta} \mathcal{G}(\varphi)\right\|_{0}+\left\|G_{1, \beta} \mathcal{G}(\varphi)\right\|_{s}\right)
\end{aligned}
$$

and, by Theorem 6.9 and further interpolation,

$$
\left\|G_{1, \beta}\left(\mathcal{G}(\varphi)\left(1+\dot{e}_{0}\right)\right)\right\|_{s} \leq c_{\beta, s}\left(M_{5}\right)\left(\|w\|_{\beta+6}\|\varphi\|_{s}+\|w\|_{s+\beta+6}\|\varphi\|_{0}\right) .
$$

The outcome is that, when $\mathcal{G}$ is any Q1D operator of order $(1, \beta)$ on $H_{\text {ut }}^{s}$ for $0 \leq \beta+s \leq m-6$, the operator $\varphi \mapsto \partial_{\xi} \partial_{\tau}^{-1}\left(\mathcal{G}(\varphi) \circ Q^{-1}\right)$ is a sum of the form

$$
\sum_{j} R_{j}\left(\mathcal{G}_{j}(\varphi) \circ Q^{-1}\right)
$$


where $\mathcal{G}_{j}$ is a Q1D operator of order either $(0, \beta-1)$ or $(1, \beta)$ on $H_{\text {㛎 }}^{s}$ for $0 \leq$ $\beta+s \leq m-6$, which satisfies

$$
\begin{aligned}
& \mathcal{G}_{1}(\varphi)=\partial_{y} \partial_{t}^{-1}\left(\mathcal{G}(\varphi)\left(1+\dot{e}_{0}\right)\right) \text { of order }(0, \beta-1), \\
& \mathcal{G}_{2}(\varphi)=\pi_{1}\left[\partial_{y}\left(\mathcal{G}(\varphi)\left(1+\dot{e}_{0}\right)\right)\right] \text { of order }(0, \beta-1), \\
& \mathcal{G}_{3}(\varphi)=\mathcal{G}(\varphi)\left(1+\dot{e}_{0}\right) \text { of order }(1, \beta)
\end{aligned}
$$

$\left\|G_{0, \beta-1} \mathcal{G}_{1}(\varphi)\right\|_{s}+\left\|G_{0, \beta-1} \mathcal{G}_{2}(\varphi)\right\|_{s}+\left\|G_{1, \beta} \mathcal{G}_{3}(\varphi)\right\|_{s} \leq c_{\beta, s}\left\|G_{1, \beta}\left(\mathcal{G}(\varphi)\left(1+\dot{e}_{0}\right)\right)\right\|_{s}$ and the operators $R_{j}$ are given by

$$
\begin{array}{ll}
R_{1}=\left\{r_{1} \cdot\right\} \circ\left(\mathbb{I}-\pi_{1}\right), & r_{1}=\varrho^{\prime} \in C_{\text {吅 }}^{m-4}, \\
R_{2}=\left\{r_{2} \cdot\right\}, & r_{2}=-\varrho^{\prime} \ell \in C_{\text {吅 }}^{m-4}, \\
R_{3}=\left(\mathbb{I}-\pi_{1}\right) \circ\left\{r_{3} \cdot\right\}, & r_{3}=-\ell_{\xi} \in C_{\text {吅 }}^{m-5},
\end{array}
$$

in which $\{h \cdot\}$ denotes the operator of multiplication by $h$, and

$$
\left\|r_{1}\right\|_{C^{l+1}}+\left\|r_{2}\right\|_{C^{l+1}}+\left\|r_{3}\right\|_{C^{l}} \leq c_{l}\left(M_{4}\right)\|w\|_{l+5}
$$

The identity

$$
\begin{aligned}
\partial_{\tau}^{-1}\left(r \mathcal{G} \circ Q^{-1}\right) & \\
=\left(\left(\mathbb{I}-\pi_{1}\right)\right. & \left.\{r \cdot\}-\partial_{\tau}^{-1}\{\dot{r} \cdot\}\right)\left(\left(\mathbb{I}-\pi_{1}\right)\left\{\partial_{t}^{-1}\left(\mathcal{G}(\varphi)\left(1+\dot{e}_{0}\right)\right) \circ Q^{-1}\right\}\right) \\
& +\left(\partial_{\tau}^{-1} r-\left(\mathbb{I}-\pi_{1}\right)\{r l \cdot\}+\partial_{\tau}^{-1}\{\dot{r} l \cdot\}\right) \pi_{1}\left(\mathcal{G}(\varphi)\left(1+\dot{e}_{0}\right)\right) \circ \varrho,
\end{aligned}
$$

follows from (L.4) and Lemma M.2, and leads (via Lemmas G.1, G.3 and (L.7)) to the basic estimate

$$
\begin{aligned}
\left\|G_{1,0}\left(r \mathcal{G}(\varphi) \circ Q^{-1}\right)\right\|_{s} \leq c_{s}\left(M_{5}\right)\left\{\left\|G_{1,0} \mathcal{G}(\varphi)\right\|_{s}\left(\|r\|_{C^{0}}+\|\dot{r}\|_{C^{0}}\right)\right. \\
\left.\quad+\left(\left(\|r\|\left\|_{C^{0}}+\right\| \dot{r} \|_{C^{0}}\right)\|w\|_{s+5}+\|r\|_{C^{s}}+\|\dot{r}\|_{C^{s}}\right)\left\|G_{1,0} \mathcal{G}(\varphi)\right\|_{0}\right\}
\end{aligned}
$$

Now (L.10) leads immediately to the observation that

$$
\begin{aligned}
\left\|G_{0, \beta-1} \mathcal{G}_{1}(\varphi)\right\|_{s}+ & \left\|G_{0, \beta-1} \mathcal{G}_{2}(\varphi)\right\|_{s}+\left\|G_{1, \beta} \mathcal{G}_{3}(\varphi)\right\|_{s} \\
& \leq c_{\beta, s}\left(M_{5}\right)\left(\|w\|_{\beta+6}\|\varphi\|_{s}+\|w\|_{s+\beta+6}\|\varphi\|_{0}\right) .
\end{aligned}
$$

We then obtain that

$$
\begin{aligned}
\left\|G_{1,0} \mathcal{G}_{1}(\varphi)\right\|_{s}+\left\|G_{1,0} \mathcal{G}_{2}(\varphi)\right\|_{s} & \leq c_{s}\left(M_{5}\right)\left(\|w\|_{7}\|\varphi\|_{s}+\|w\|_{s+7}\|\varphi\|_{0}\right), \\
\left\|G_{1,0} \mathcal{G}_{3}(\varphi)\right\|_{s} & \leq c_{s}\left(M_{5}\right)\left(\|w\|_{6}\|\varphi\|_{s}+\|w\|_{s+6}\|\varphi\|_{0}\right) .
\end{aligned}
$$

Since

$$
\dot{r}_{1}, \dot{r}_{2}, \dot{\ell} \in C_{\text {吅 }}^{m-4}, \quad \dot{r}_{3} \in C_{\text {仲 }}^{m-5},
$$

standard interpolation shows that

$$
\left\|G_{1,0} R_{j} \mathcal{G}_{j}(\varphi) \circ Q^{-1}\right\|_{s} \leq c_{s}\left(M_{5}\right)\left(\|w\|_{7}\|\varphi\|_{s}+\|w\|_{s+7}\|\varphi\|_{0}\right) \text { for } j=1,2,3
$$


This yields the claim of the lemma for $\beta=1$ :

$$
\left\|G_{2,1} \mathcal{G}(\varphi) \circ Q^{-1}\right\|_{s} \leq c_{s}\left(M_{5}\right)\left(\|w\|_{7}\|\varphi\|_{s}+\|w\|_{s+7}\|\varphi\|_{0}\right) .
$$

If we now consider $\left(\partial_{\xi} \partial_{\tau}^{-1}\right)^{2}\left(\mathcal{G}(\varphi) \circ Q^{-1}\right)$, we obtain

$$
\left(\partial_{\xi} \partial_{\tau}^{-1}\right)^{2}\left(\mathcal{G}(\varphi) \circ Q^{-1}\right)=\sum_{j, l} R_{j l}\left(\mathcal{G}_{j l}(\varphi) \circ Q^{-1}\right)
$$

where

$$
\begin{aligned}
& \mathcal{G}_{11}(\varphi)=\partial_{y} \partial_{t}^{-1}\left(\mathcal{G}_{1}(\varphi)\left(1+\dot{e}_{0}\right)\right) \text { of order }(0, \beta-2) \\
& \mathcal{G}_{12}(\varphi)=\pi_{1}\left[\partial_{y}\left(\mathcal{G}_{1}(\varphi)\left(1+\dot{e}_{0}\right)\right)\right] \text { of order }(0, \beta-2) \\
& \mathcal{G}_{13}(\varphi)=\mathcal{G}_{1}(\varphi)\left(1+\dot{e}_{0}\right) \text { of order }(0, \beta-1) \\
& \mathcal{G}_{14}(\varphi)=\partial_{t}^{-1}\left(\mathcal{G}_{1}(\varphi)\left(1+\dot{e}_{0}\right)\right) \text { of order }(0, \beta-1) \\
& \mathcal{G}_{15}(\varphi)=\pi_{1}\left(\mathcal{G}_{1}(\varphi)\left(1+\dot{e}_{0}\right)\right) \text { of order }(0, \beta-1) \\
& \mathcal{G}_{21}(\varphi)=\partial_{y}\left(\mathcal{G}_{2}(\varphi)\left(1+\dot{e}_{0}\right)\right) \text { of order }(0, \beta-2) \\
& \mathcal{G}_{22}(\varphi)=\mathcal{G}_{2}(\varphi) \text { of order }(0, \beta-1) \\
& \mathcal{G}_{31}(\varphi)=\partial_{y} \partial_{t}^{-1}\left(\mathcal{G}_{3}(\varphi)\left(1+\dot{e}_{0}\right)\right) \text { of order }(0, \beta-1) \\
& \mathcal{G}_{32}(\varphi)=\pi_{1}\left[\partial_{y}\left(\mathcal{G}_{3}(\varphi)\left(1+\dot{e}_{0}\right)\right)\right] \text { of order }(0, \beta-1) \\
& \mathcal{G}_{33}(\varphi)=\mathcal{G}_{3}(\varphi)\left(1+\dot{e}_{0}\right) \text { of order }(1, \beta) \\
& \mathcal{G}_{34}(\varphi)=\partial_{t}^{-1}\left(\mathcal{G}_{3}(\varphi)\left(1+\dot{e}_{0}\right)\right) \text { of order }(0, \beta) \\
& \mathcal{G}_{35}(\varphi)=\pi_{1}\left(\mathcal{G}_{3}(\varphi)\left(1+\dot{e}_{0}\right)\right) \text { of order }(0, \beta)
\end{aligned}
$$

and, in an obvious abbreviated notation, operators $R_{i j}$ are defined by

$$
\begin{aligned}
& R_{11}=R_{1}^{2} \in C_{\text {仲 }}^{m-4} \\
& R_{12}=R_{1} r_{2} \in C_{\text {如 }}^{m-4} \\
& R_{13}=R_{1} r_{3} \in C_{\text {比 }}^{m-5} \\
& R_{14}=R_{1}^{\prime} \in C_{\text {仲 }}^{m-5} \\
& R_{15}=-R_{1}^{\prime} \ell \in C_{\text {仲 }}^{m-5} \\
& R_{21}=\varrho^{\prime} \partial_{\tau}^{-1} r_{2} \in C_{\text {如 }}^{m-4} \\
& R_{22}=\partial_{\xi} \partial_{\tau}^{-1} r_{2} \in C_{\text {如 }}^{m-5} \\
& R_{31}=\left\{R_{3} R_{1} \cdot\right\}-\partial_{\tau}^{-1}\left\{\dot{R}_{3} R_{1} \cdot\right\} \in C_{\text {七七 }}^{m-5} \\
& R_{32}=\left\{R_{3} r_{2} \cdot\right\}-\partial_{\tau}^{-1}\left\{\dot{R}_{3} r_{2} \cdot\right\}+\varrho^{\prime} \partial_{\tau}^{-1} R_{3} \in C_{\text {如 }}^{m-5} \\
& R_{33}=\left\{R_{3}^{2} \cdot\right\}-\partial_{\tau}^{-1}\left\{\dot{R}_{3} R_{3} \cdot\right\} \in C_{\text {如 }}^{m-5} \\
& R_{34}=\left\{\partial_{\xi} R_{3} \cdot\right\}-\partial_{\tau}^{-1}\left\{\partial_{\xi} \dot{R}_{3} \cdot\right\}\left(\mathbb{I}-\pi_{1}\right) \in C_{\text {如 }}^{m-6} \\
& R_{35}=\partial_{\xi} \partial_{\tau}^{-1} R_{3}-\left(\mathbb{I}-\pi_{1}\right)\left\{l \partial_{\xi} R_{3} \cdot\right\}-\partial_{\tau}^{-1}\left\{l \partial_{\xi} \dot{R}_{3} \cdot\right\} \in C_{\text {切 }}^{m-6} \text {. }
\end{aligned}
$$


From (L.9) for $\mathcal{G}_{j}$, (L.10) and interpolation, we obtain the estimate

$$
\begin{aligned}
\left\|G_{0, \beta-2} \mathcal{G}_{11}(\varphi)\right\|_{s}+\left\|G_{0, \beta-2} \mathcal{G}_{12}(\varphi)\right\|_{s}+\left\|G_{0, \beta-2} \mathcal{G}_{21}(\varphi)\right\|_{s} \\
\quad+\left\|G_{0, \beta-1} \mathcal{G}_{13}(\varphi)\right\|_{s}+\left\|G_{0, \beta-1} \mathcal{G}_{14}(\varphi)\right\|_{s}+\left\|G_{0, \beta-1} \mathcal{G}_{15}(\varphi)\right\|_{s} \\
\quad+\left\|G_{0, \beta-1} \mathcal{G}_{31}(\varphi)\right\|_{s}+\left\|G_{0, \beta-1} \mathcal{G}_{32}(\varphi)\right\|_{s}+\left\|G_{1, \beta} \mathcal{G}_{33}(\varphi)\right\|_{s} \\
\quad+\left\|G_{0, \beta} \mathcal{G}_{34}(\varphi)\right\|_{s}+\left\|G_{0, \beta} \mathcal{G}_{35}(\varphi)\right\|_{s} \\
\leq c_{\beta, s}\left\{\left\|G_{0, \beta-1}\left(\mathcal{G}_{1}(\varphi)\left(1+\dot{e}_{0}\right)\right)\right\|_{s}\right. \\
\left.\quad+\left\|G_{0, \beta-1}\left(\mathcal{G}_{2}(\varphi)\left(1+\dot{e}_{0}\right)\right)\right\|_{s}+\left\|G_{1, \beta}\left(\mathcal{G}_{3}(\varphi)\left(1+\dot{e}_{0}\right)\right)\right\|_{s}\right\} \\
\leq c_{\beta, s}\left(M_{5}\right)\left(\|w\|_{\beta+6}\|\varphi\|_{s}+\|w\|_{s+\beta+6}\|\varphi\|_{0}\right),
\end{aligned}
$$

where the coefficients satisfy

$$
\begin{array}{r}
\left\|R_{11}\right\|_{C^{l+1}}+\left\|R_{12}\right\|_{C^{l+1}}+\left\|R_{21}\right\|_{C^{l+1}}+\left\|R_{13}\right\|_{C^{l}}+\left\|R_{14}\right\|_{C^{l}}+\left\|R_{15}\right\|_{C^{l}} \\
+\left\|R_{22}\right\|_{C^{l}}+\left\|R_{31}\right\|_{C^{l}}+\left\|R_{32}\right\|_{C^{l}}+\left\|R_{33}\right\|_{C^{l}}+\left\|R_{34}\right\|_{C^{l-1}}+\left\|R_{35}\right\|_{C^{l-1}} \\
\leq c_{l}\left(M_{4}\right)\|w\|_{l+5} .
\end{array}
$$

Let us make the following inductive assumption: for $k \geq 2$

$$
\left(\partial_{\xi} \partial_{\tau}^{-1}\right)^{k}\left(\mathcal{G}(\varphi) \circ Q^{-1}\right)=\sum_{\substack{0 \leq j \leq k+1 \\ 1 \leq l \leq L(k)}} R_{j, l}^{(k)}\left(\mathcal{G}_{j, l}^{(k)}(\varphi) \circ Q^{-1}\right)
$$

for some finite $L(k)$, and on $H_{\text {蛞 }}^{s}$

$$
\begin{gathered}
\mathcal{G}_{j, l}^{(k)} \quad \text { Q1D of order }(0, \beta-k+j) \text { for } 0 \leq j \leq k \\
\mathcal{G}_{k+1, l}^{(k)} \quad \text { Q1D of order }(1, \beta) \text { for } j=k+1, \\
R_{j, l}^{(k)} \in C_{\text {如 }}^{m-2-j-k} \text { for } 0 \leq j \leq k, \quad R_{k+1, l}^{(k)} \in C_{\text {如 }}^{m-2-2 k} \text { for } j=k+1,
\end{gathered}
$$

with estimates

$$
\begin{aligned}
\| G_{0, \beta-k+j} & \mathcal{G}_{j, l}^{(k)}(\varphi)\left\|_{s}+\right\| G_{1, \beta} \mathcal{G}_{k+1, l}^{(k)}(\varphi) \|_{s} \\
& \leq c_{\beta, s}\left(M_{5}\right)\left(\|w\|_{\beta+6}\|\varphi\|_{s}+\|w\|_{s+\beta+6}\|\varphi\|_{0}\right), \\
\left\|R_{j, l}^{(k)}\right\|_{C^{s}} & \leq c_{s}\left(M_{4}\right)\|w\|_{s+j+k+2} \text { for } 0 \leq j \leq k, \\
\left\|R_{k+1, l}^{(k)}\right\|_{C^{s}} & \leq c_{s}\left(M_{4}\right)\|w\|_{s+2 k+2} \text { for } j=k+1 .
\end{aligned}
$$

We note that the anticipated smoothness of the coefficients $R_{j, l}^{(k)}$ is not as good as might have been expected from the cases $k=0,1$. This is because in each later step we can loose two derivatives. In the step from $k$ to $k+1$, the term $\left(\partial_{\xi} \partial_{\tau}^{-1}\right) R_{j, l}^{(k)}\left(\mathcal{G}_{j, l}^{(k)}(\varphi) \circ Q^{-1}\right)$ leads to terms of the form $r^{(k+1)}\left(\mathcal{G}^{(k+1)}(\varphi) \circ Q^{-1}\right)$ where, for $0 \leq j \leq k$, either

$$
\mathcal{G}^{(k+1)} \text { has order }(0, \beta-k+j-1) \text { and } R^{(k+1)} \in C_{\text {如 }}^{m-2-j-k-1}
$$


Or

$$
\mathcal{G}^{(k+1)} \text { has order }(0, \beta-k+j) \text { and } R^{(k+1)} \in C_{\text {如 }}^{m-2-j-k-2},
$$

and estimates analogous to (L.13) hold. If in (L.12), with $k+1$ instead of $k$ we use $j^{\prime}$ instead of $j$, then in the first case $j^{\prime}=j$ and in the second $j^{\prime}=j+1$. Therefore for $j=k+1$, we obtain either

$$
\mathcal{G}^{(k+1)} \text { of order }(0, \beta-1), \quad R^{(k+1)} \in C_{\text {仲 }}^{m-2-2 k-1}
$$

or

$$
\mathcal{G}^{(k+1)} \text { of order }(1, \beta), \quad R^{(k+1)} \in C_{\text {㲺 }}^{m-2-2 k-2},
$$

which shows that (L.12) for $k$ implies (L.12) for $k+1$, since in the first case $j^{\prime} \leq k+1$, and in the second case $j^{\prime}=k+2$. From (L.11), the estimates for $R_{j, l}^{(k)}$ and the estimates for $G_{1,0} \mathcal{G}_{j, l}^{(k)}(\varphi)$,

$$
\begin{aligned}
\left\|G_{1,0} \mathcal{G}_{j, l}^{(k)}(\varphi)\right\|_{s} & \leq c_{\beta, s}\left(M_{5}\right)\left(\|w\|_{k+6-j}\|\varphi\|_{s}+\|w\|_{s+k+6-j}\|\varphi\|_{0}\right) \\
\left\|G_{1,0} \mathcal{G}_{k+1, l}^{(k)}(\varphi)\right\|_{s} & \leq c_{\beta, s}\left(M_{5}\right)\left(\|w\|_{6}\|\varphi\|_{s}+\|w\|_{s+6}\|\varphi\|_{0}\right)
\end{aligned}
$$

which follows from (L.13) with $\beta=k-j$, we obtain, for $k \geq 2$, after standard interpolation,

$$
\left\|G_{k+1, k} \mathcal{G}(\varphi) \circ Q^{-1}\right\|_{s} \leq c_{\beta, s}\left(M_{5}\right)\left(\|w\|_{2 k+4}\|\varphi\|_{s}+\|w\|_{s+2 k+4}\|\varphi\|_{0}\right) .
$$

By induction on $k \in\{1, \cdots, \beta\}$, when combined with (L.3) and (L.1) this gives the required result for $\mathcal{G}_{0}$ in Lemma 7.2 .

\section{Proof of Theorem 7.5}

From (7.16),

$$
\vartheta=\left\{1+\alpha_{0}+\beta_{0} \mathcal{H}+\left(\alpha_{1}+\beta_{1} \mathcal{H}\right) \partial_{\tau}^{-1}+\left(\alpha_{2}+\beta_{2} \mathcal{H}\right) \partial_{\tau}^{-2}\right\} \theta
$$

where $\alpha_{j}, \beta_{j}, j=0,1,2$ are functions which are doubly periodic in $\xi$ and $\tau$ to be determined below. We require that the $\alpha_{j}$ are even in $\xi$, the $\beta_{j}$ are odd in $\xi$, that $\alpha_{0}, \beta_{0}, \alpha_{2}, \beta_{2}$ are even in $\tau$, and that $\alpha_{1}, \beta_{1}$ are odd in $\tau$. Let $\mathcal{L}$ denote the linear operator

$$
\mathcal{L}=\partial_{\tau \tau}-\left(1+\beta^{(0)}\right) \mathcal{H} \partial_{\xi}
$$

Then (7.5) is of the form

$$
\mathcal{L} \theta+(\gamma+\delta \mathcal{H}) \partial_{\tau} \theta+\alpha \mathcal{H} \theta+\mathcal{G}_{0} \theta=g
$$

Note the following commutator relations satisfied by $\mathcal{L}$ :

$$
\begin{aligned}
\mathcal{L}\left(\left(\alpha_{0}\right.\right. & \left.\left.+\beta_{0} \mathcal{H}\right) \theta\right)-\left(\alpha_{0}+\beta_{0} \mathcal{H}\right) \mathcal{L} \theta=2\left(\dot{\alpha}_{0}+\dot{\beta}_{0} \mathcal{H}\right) \partial_{\tau} \theta \\
& +\left\{\left(\ddot{\alpha}_{0}+\ddot{\beta}_{0} \mathcal{H}+\left(1+\beta^{(0)}\right)\left(\beta_{0}^{\prime}-\alpha_{0}^{\prime} \mathcal{H}\right)\right\} \theta-\left(1+\beta^{(0)}\right) \partial_{\xi} \mathcal{S}_{\alpha_{0}, \beta_{0}} \theta\right.
\end{aligned}
$$

where the new $\xi$-smoothing operator $\mathcal{S}_{\alpha_{0}, \beta_{0}}$ (recall $\mathcal{S}_{\omega}$ in Lemma 6.3) is defined by

$$
\mathcal{S}_{p, q} f \stackrel{\text { def }}{=} \mathcal{S}_{p} f+\mathcal{S}_{q} \mathcal{H} f+q \pi_{0} f
$$


Remark M.1. Note, from Lemma 6.5, that if $p, q \in H^{m}$ then $\mathcal{S}_{p, q}$ is a Q1D operator of order $(0, \beta)$ on $H_{\text {㛎 }}^{s}$ when $0 \leq \beta+s \leq m-3$. Moreover we have the estimate

$\left\|G_{0, \beta} \mathcal{S}_{p, q} f\right\|_{s} \leq c_{\beta, s}\left\{\left(\|p\|_{C^{\beta+s+1}}+\|q\|_{C^{\beta+s+1}}\right)\|f\|_{0}+\left(\|p\|_{C^{\beta+1}}+\|q\|_{C^{\beta+1}}\right)\|f\|_{s}\right\}$.

Since $\mathcal{L}$ commutes with $\partial_{\tau}^{-1}$,

$$
\begin{aligned}
& \mathcal{L}\left(\left(\alpha_{1}+\beta_{1} \mathcal{H}\right) \partial_{\tau}^{-1} \theta\right)-\left(\alpha_{1}+\beta_{1} \mathcal{H}\right) \partial_{\tau}^{-1} \mathcal{L} \theta=2\left(\dot{\alpha}_{1}+\dot{\beta}_{1} \mathcal{H}\right)\left(1-\pi_{1}\right) \theta \\
& +\left\{\left(\ddot{\alpha}_{1}+\ddot{\beta}_{1} \mathcal{H}+\left(1+\beta^{(0)}\right)\left(\beta_{1}^{\prime}-\alpha_{1}^{\prime} \mathcal{H}\right)\right\} \partial_{\tau}^{-1} \theta-\left(1+\beta^{(0)}\right) \partial_{\xi} \mathcal{S}_{\alpha_{1}, \beta_{1}} \partial_{\tau}^{-1} \theta\right.
\end{aligned}
$$

and

$$
\begin{aligned}
& \mathcal{L}\left(\left(\alpha_{2}+\beta_{2} \mathcal{H}\right) \partial_{\tau}^{-2} \theta\right)-\left(\alpha_{2}+\beta_{2} \mathcal{H}\right) \partial_{\tau}^{-2} \mathcal{L} \theta=2\left(\dot{\alpha}_{2}+\dot{\beta}_{2} \mathcal{H}\right) \partial_{\tau}^{-1} \theta \\
+ & \left\{\left(\ddot{\alpha}_{2}+\ddot{\beta}_{2} \mathcal{H}+\left(1+\beta^{(0)}\right)\left(\beta_{2}^{\prime}-\alpha_{2}^{\prime} \mathcal{H}\right)\right\} \partial_{\tau}^{-2} \theta-\left(1+\beta^{(0)}\right) \partial_{\xi} \mathcal{S}_{\alpha_{2}, \beta_{2}} \partial_{\tau}^{-2} \theta .\right.
\end{aligned}
$$

To calculate the effect of changing variables on the other terms in (M.2) we need the following simple identity.

Lemma M.2. For $\omega \in C_{\text {如 }}^{m}, m \geq 1$ and $f \in L_{\text {如, }}^{2}$,

$$
\partial_{\tau}^{-1}(\omega f)=\omega \partial_{\tau}^{-1} f+\left(\partial_{\tau}^{-1} \omega\right) \pi_{1} f-\pi_{1}\left(\omega \partial_{\tau}^{-1} f\right)-\partial_{\tau}^{-1}\left(\dot{\omega} \partial_{\tau}^{-1} f\right) .
$$

Proof. Since $\partial_{\tau}$ and $\partial_{\tau}^{-1}$ commute,

$$
\partial_{\tau}^{-1}\left(\dot{\omega} \partial_{\tau}^{-1} f\right)+\partial_{\tau}^{-1}\left\{\omega\left(1-\pi_{1}\right) f\right\}=\partial_{\tau} \partial_{\tau}^{-1}\left(\omega \partial_{\tau}^{-1} f\right)=\left(1-\pi_{1}\right)\left(\omega \partial_{\tau}^{-1} f\right),
$$

and the result follows.

This then leads to the following identities:

$$
\begin{aligned}
& -\left(1+\alpha_{0}+\beta_{0} \mathcal{H}\right)(\gamma+\delta \mathcal{H}) \partial_{\tau} \theta \\
& =\left\{\beta_{0} \delta-\left(1+\alpha_{0}\right) \gamma-\left(\beta_{0} \gamma+\left(1+\alpha_{0}\right) \delta\right) \mathcal{H}\right\} \partial_{\tau} \theta-\beta_{0} \mathcal{S}_{\gamma, \delta} \partial_{\tau} \theta \\
& \quad-\left(1+\alpha_{0}+\beta_{0} \mathcal{H}\right) \alpha \mathcal{H} \theta=\left\{\beta_{0} \alpha-\left(1+\alpha_{0}\right) \alpha \mathcal{H}\right\} \theta-\beta_{0} \mathcal{S}_{0, \alpha} \theta \\
& -\left(\alpha_{1}+\beta_{1} \mathcal{H}\right) \partial_{\tau}^{-1}(\gamma+\delta \mathcal{H}) \partial_{\tau} \theta=-\left(\alpha_{1}+\beta_{1} \mathcal{H}\right)\left\{(\gamma+\delta \mathcal{H})\left(1-\pi_{1}\right) \theta-(\dot{\gamma}+\dot{\delta} \mathcal{H}) \partial_{\tau}^{-1} \theta\right. \\
& \left.\quad-2 \pi_{1}\left((\gamma+\delta \mathcal{H})\left(1-\pi_{1}\right) \theta\right)+\partial_{\tau}^{-1}\left((\ddot{\gamma}+\ddot{\delta} \mathcal{H}) \partial_{\tau}^{-1} \theta\right)\right\} \\
& =\left\{\beta_{1} \delta-\alpha_{1} \gamma-\left(\beta_{1} \gamma+\alpha_{1} \delta\right) \mathcal{H}\right\}\left(1-\pi_{1}\right) \theta-\beta_{1} \mathcal{S}_{\gamma, \delta}\left(1-\pi_{1}\right) \theta \\
& +\left\{\alpha_{1} \dot{\gamma}-\beta_{1} \dot{\delta}+\left(\beta_{1} \dot{\gamma}+\alpha_{1} \dot{\delta}\right) \mathcal{H}\right\} \partial_{\tau}^{-1} \theta+\beta_{1} \mathcal{S}_{\dot{\gamma}, \dot{\delta}} \partial_{\tau}^{-1} \theta \\
& +\left\{\beta_{1} \ddot{\delta}-\alpha_{1} \ddot{\gamma}-\left(\beta_{1} \ddot{\gamma}+\alpha_{1} \ddot{\delta}\right) \mathcal{H}\right\} \partial_{\tau}^{-2} \theta-\beta_{1} \mathcal{S}_{\ddot{\gamma}, \ddot{\delta}} \partial_{\tau}^{-2} \theta \\
& +\left(\alpha_{1}+\beta_{1} \mathcal{H}\right)\left\{3 \pi_{1}\left[(\gamma+\delta \mathcal{H})\left(1-\pi_{1}\right) \theta\right]+\partial_{\tau}^{-1}\left[(\dddot{\gamma}+\dddot{\delta} \mathcal{H}) \partial_{\tau}^{-2} \theta\right]\right\}
\end{aligned}
$$


and

$$
\begin{gathered}
-\left(\alpha_{1}+\beta_{1} \mathcal{H}\right) \partial_{\tau}^{-1} \alpha \mathcal{H} \theta \\
=-\left(\alpha_{1}+\beta_{1} \mathcal{H}\right)\left\{\alpha \mathcal{H} \partial_{\tau}^{-1} \theta+\left(\partial_{\tau}^{-1} \alpha\right) \mathcal{H} \pi_{1} \theta-\pi_{1}\left[\alpha \mathcal{H} \partial_{\tau}^{-1} \theta\right]-\partial_{\tau}^{-1}\left[\dot{\alpha} \mathcal{H} \partial_{\tau}^{-1} \theta\right]\right\} \\
=\left\{\beta_{1} \alpha-\alpha_{1} \alpha \mathcal{H}-\beta_{1} \mathcal{S}_{0, \alpha}\right\} \partial_{\tau}^{-1} \theta+\left\{-\beta_{1} \dot{\alpha}+\alpha_{1} \dot{\alpha} \mathcal{H}+\beta_{1} \mathcal{S}_{0, \dot{\alpha}}\right\} \partial_{\tau}^{-2} \theta \\
\quad-\left(\alpha_{1}+\beta_{1} \mathcal{H}\right)\left\{\partial_{\tau}^{-1} \alpha \mathcal{H} \pi_{1} \theta-2 \pi_{1}\left[\alpha \mathcal{H} \partial_{\tau}^{-1} \theta\right]+\partial_{\tau}^{-1}\left[\ddot{\alpha} \mathcal{H} \partial_{\tau}^{-2} \theta\right]\right\} .
\end{gathered}
$$

Similar repeated applications of the formula in Lemma M.2 yields

$$
\begin{aligned}
&-\left(\alpha_{2}+\beta_{2} \mathcal{H}\right) \partial_{\tau}^{-2}(\gamma+\delta \mathcal{H}) \partial_{\tau} \theta \\
&=-\left(\alpha_{2}+\beta_{2} \mathcal{H}\right)\left\{(\gamma+\delta \mathcal{H}) \partial_{\tau}^{-1} \theta-\pi_{1}\left[(\gamma+\delta \mathcal{H}) \partial_{\tau}^{-1} \theta\right]\right. \\
&\left.-2 \partial_{\tau}^{-1}(\dot{\gamma}+\dot{\delta} \mathcal{H}) \partial_{\tau}^{-1} \theta+\partial_{\tau}^{-2}\left[(\ddot{\gamma}+\ddot{\delta} \mathcal{H}) \partial_{\tau}^{-1} \theta\right]\right\} \\
&=\left\{\beta_{2} \delta-\alpha_{2} \gamma-\left(\beta_{2} \gamma+\alpha_{2} \delta\right) \mathcal{H}\right\} \partial_{\tau}^{-1} \theta-\beta_{2} \mathcal{S}_{\gamma, \delta} \partial_{\tau}^{-1} \theta \\
&+ 2\left\{\alpha_{2} \dot{\gamma}-\beta_{2} \dot{\delta}+\left(\beta_{2} \dot{\gamma}+\alpha_{2} \dot{\delta}\right) \mathcal{H}\right\} \partial_{\tau}^{-2} \theta+2 \beta_{2} \mathcal{S}_{\dot{\gamma}, \dot{\delta}} \partial_{\tau}^{-2} \theta \\
&+\left(\alpha_{2}+\beta_{2} \mathcal{H}\right)\left\{3 \pi_{1}\left[(\gamma+\delta \mathcal{H}) \partial_{\tau}^{-1} \theta\right]-3 \partial_{\tau}^{-1}\left[(\ddot{\gamma}+\ddot{\delta} \mathcal{H}) \partial_{\tau}^{-2} \theta\right]+\partial_{\tau}^{-2}\left[(\dddot{\gamma}+\dddot{\delta} \mathcal{H}) \partial_{\tau}^{-2} \theta\right\} ;\right.
\end{aligned}
$$

and

$$
\begin{gathered}
-\left(\alpha_{2}+\beta_{2} \mathcal{H}\right) \partial_{\tau}^{-2} \alpha \mathcal{H} \theta=-\left(\alpha_{2}+\beta_{2} \mathcal{H}\right)\left\{\left(1-\pi_{1}\right)\left[\alpha \mathcal{H} \partial_{\tau}^{-2} \theta\right]\right. \\
\left.-2 \partial_{\tau}^{-1}\left[\dot{\alpha} \mathcal{H} \partial_{\tau}^{-2} \theta\right]+\partial_{\tau}^{-2}\left[\alpha \mathcal{H} \pi_{1} \theta+\ddot{\alpha} \mathcal{H} \partial_{\tau}^{-2} \theta\right]\right\} \\
=\left\{\beta_{2} \alpha-\alpha_{2} \alpha \mathcal{H}\right\} \partial_{\tau}^{-2} \theta-\beta_{2} \mathcal{S}_{0, \alpha} \partial_{\tau}^{-2} \theta \\
+\left(\alpha_{2}+\beta_{2} \mathcal{H}\right)\left\{\pi_{1}\left[\alpha \mathcal{H} \partial_{\tau}^{-2} \theta\right]-\partial_{\tau}^{-2} \alpha \mathcal{H} \pi_{1} \theta\right. \\
\left.\quad+2 \partial_{\tau}^{-1}\left[\dot{\alpha} \mathcal{H} \partial_{\tau}^{-2} \theta\right]-\partial_{\tau}^{-2}\left[\ddot{\alpha} \mathcal{H} \partial_{\tau}^{-2} \theta\right]\right\} .
\end{gathered}
$$

Now the object is to find coefficients in formula (M.1) and a constant $\kappa^{(0)}$ which ensure that, in the resulting expression for $\mathcal{L} \vartheta-\kappa^{(0)} \vartheta$, the coefficients of $\partial_{\tau} \theta, \theta, \partial_{\tau}^{-1} \theta$ are zero when $\theta$ satisfies (M.2). The commutator relations (M.3), (M.5), (M.6), followed by a substitution for $\mathcal{L} \theta$ from (M.2) with the identities displayed above, now leads to the following system for the unknown functions $\alpha_{j}, \beta_{j} j=0,1,2$ :

$$
\begin{gathered}
2 \dot{\alpha}_{0}-\left(1+\alpha_{0}\right) \gamma+\beta_{0} \delta=0, \\
2 \dot{\beta}_{0}-\beta_{0} \gamma-\left(1+\alpha_{0}\right) \delta=0, \\
\ddot{\alpha}_{0}+\left(1+\beta^{(0)}\right) \beta_{0}^{\prime}-\left(1+\alpha_{0}\right) \kappa^{(0)}+\beta_{0} \alpha=0, \\
2 \dot{\alpha}_{1}-\alpha_{1} \gamma+\beta_{1} \delta+\alpha_{0}=0, \\
2 \dot{\beta}_{1}-\beta_{1} \gamma-\alpha_{1} \delta+\ddot{\beta}_{0}-\left(1+\beta^{(0)}\right) \alpha_{0}^{\prime}-\beta_{0} \kappa^{(0)}-\left(1+\alpha_{0}\right)=0 \\
2 \dot{\alpha}_{2}-\alpha_{2} \gamma+\beta_{2} \delta+\ddot{\alpha}_{1}+\left(1+\beta^{(0)}\right) \beta_{1}^{\prime}-\alpha_{1} \kappa^{(0)}+\beta_{1} \alpha+\alpha_{1} \dot{\gamma}-\beta_{1} \dot{\delta}=0, \\
2 \dot{\beta}_{2}-\beta_{2} \gamma-\alpha_{2} \delta+\ddot{\beta}_{1}-\left(1+\beta^{(0)}\right) \alpha_{1}^{\prime}-\beta_{1} \kappa^{(0)}-\alpha_{1} \alpha+\beta_{1} \dot{\gamma}+\alpha_{1} \dot{\delta}=0 .
\end{gathered}
$$


Introducing the complex functions $Z_{j}$ defined by

$$
Z_{0}=1+\alpha_{0}+i \beta_{0}, \quad Z_{1}=\alpha_{1}+i \beta_{1}, \quad Z_{2}=\alpha_{2}+i \beta_{2},
$$

the above linear system becomes

$$
\begin{gathered}
2 \dot{Z}_{0}-(\gamma+i \delta) Z_{0}=0 \\
2 \dot{Z}_{1}-(\gamma+i \delta) Z_{1}+\ddot{Z}_{0}-i\left(1+\beta^{(0)}\right) Z_{0}^{\prime}-\left(\kappa^{(0)}+i \alpha\right) Z_{0}=0, \\
2 \dot{Z}_{2}-(\gamma+i \delta) Z_{2}+\ddot{Z}_{1}-i\left(1+\beta^{(0)}\right) Z_{1}^{\prime}-\left(\kappa^{(0)}+i \alpha\right) Z_{1}+(\dot{\gamma}+i \dot{\delta}) Z_{1}=0 .
\end{gathered}
$$

The solution of (M.7) is

$$
Z_{0}(\xi, \tau)=\zeta_{0}(\xi) e^{\frac{1}{2} \int_{0}^{\tau}(\gamma(\xi, s)+i \delta(\xi, s)) d s}
$$

where

$$
\zeta_{0}(\xi)=\rho_{0}(\xi) e^{i \psi_{0}(\xi)}
$$

is, so far, arbitrary in the space $C_{\text {如 }}^{m-5, e e}+i C_{\text {如 }}^{m-5, o e}$, because $\gamma$ and $\delta$ are odd in $\tau$. It is easy to check that

$$
\ddot{Z}_{0}-i\left(1+\beta^{(0)}\right) Z_{0}^{\prime}-\left(\kappa^{(0)}+i \alpha\right) Z_{0}=\left(b_{1}+i b_{2}\right) Z_{0}
$$

where

$$
\begin{aligned}
b_{1}+i b_{2}= & \frac{1}{2}(\dot{\gamma}+i \dot{\delta})+\frac{1}{4}(\gamma+i \delta)^{2}-\left(\kappa^{(0)}+i \alpha\right) \\
& -\frac{i}{2}\left(1+\beta^{(0)}\right) \int_{0}^{\tau}\left(\gamma^{\prime}(\xi, s)+i \delta^{\prime}(\xi, s)\right) d s-i\left(1+\beta^{(0)}\right)\left(\frac{\rho_{0}^{\prime}}{\rho_{0}}+i \psi_{0}^{\prime}\right) .
\end{aligned}
$$

To solve (M.8) and (M.9) we use the variation of constants method. Let

$$
Z_{1}=V_{1} Z_{0}, \quad Z_{2}=V_{2} Z_{0}
$$

When this is substituted in (M.8), (M.10) implies that

$$
\dot{V}_{1}=-\frac{1}{2}\left(b_{1}+i b_{2}\right) \in C_{\text {吅 }}^{m-6, e e}+i C_{\text {如 }}^{m-6, o e},
$$

and, if there is to be a periodic solution $V_{1}(\xi, \tau)$, then the following compatibility condition must hold:

$$
\int_{-\pi}^{\pi}\left(b_{1}+i b_{2}\right) d \tau=0
$$

This condition implies that

$$
\begin{aligned}
\frac{\rho_{0}^{\prime}}{\rho_{0}}+i \psi_{0}^{\prime}=-\frac{1}{4 \pi} \partial_{\xi} & \left(\int_{-\pi}^{\pi} \int_{0}^{\tau}(\gamma(\xi, s)+i \delta(\xi, s)) d s d \tau\right) \\
& -\frac{i}{2 \pi\left(1+\beta^{(0)}\right)} \int_{-\pi}^{\pi}\left(\frac{1}{4}(\gamma+i \delta)^{2}-\left(\kappa^{(0)}+i \alpha\right)\right) d \tau
\end{aligned}
$$


and determines $\rho_{0}(\xi)$ and $\psi_{0}(\xi)$, respectively in $C_{b}^{m-5, e}$ and $C_{b}^{m-5, o}$, up to a scalar multiplier of $\rho_{0}$, provided the average in $\xi$ of the right hand side is 0 . The oddness in $\xi$ of $\alpha$ and $\delta$, with the evenness of $\gamma$ leads to a single condition

$$
\kappa^{(0)}=\frac{1}{4 \pi^{2}} \int_{-\pi}^{\pi} \int_{-\pi}^{\pi} \frac{1}{4}\left(\gamma^{2}-\delta^{2}\right) d \tau d \xi
$$

which determines $\kappa^{(0)}$. Then (M.13), fixing $\rho_{0}(0)=1$, yields

$$
\begin{aligned}
& \psi_{0}(\xi)=\frac{1}{2 \pi\left(1+\beta^{(0)}\right)} \int_{0}^{\xi} d s \int_{-\pi}^{\pi}\left(\frac{\delta^{2}-\gamma^{2}}{4}+\kappa^{(0)}\right)(s, \tau) d \tau-\frac{1}{4 \pi} \int_{-\pi}^{\pi} d \tau \int_{0}^{\tau} \delta(\xi, s) d s, \\
& \rho_{0}(\xi)=\exp \left\{\frac{1}{2 \pi\left(1+\beta^{(0)}\right)} \int_{0}^{\xi} d s \int_{-\pi}^{\pi}\left(-\alpha+\frac{\gamma \delta}{2}\right)(s, \tau) d \tau-\frac{1}{4 \pi} \int_{-\pi}^{\pi} d \tau \int_{0}^{\tau} \gamma(\xi, s) d s\right\},
\end{aligned}
$$

whence $Z_{0} \in C_{\text {吅 }}^{m-5, e e}+i C_{\text {吅 }}^{m-5, o e}$ and

$$
Z_{1}(\xi, \tau)=-\frac{1}{2} Z_{0}(\xi, \tau) \int_{0}^{\tau}\left(b_{1}+i b_{2}\right)(\xi, s) d s \in C_{\text {吅 }}^{m-6, e o}+i C_{\text {如 }}^{m-6, o o} .
$$

To deal with the substitution of (M.11) in (M.9) note first that, since $Z_{1}=V_{1} Z_{0}$, (M.10) and (M.12) give

$$
\ddot{Z}_{1}-i\left(1+\beta^{(0)}\right) Z_{1}^{\prime}-\left(\kappa^{(0)}+i \alpha\right) Z_{1}+(\dot{\gamma}+i \dot{\delta}) Z_{1}=\left(b_{3}+i b_{4}\right) Z_{0}
$$

$b_{3}+i b_{4}=\partial_{\tau}\left[V_{1}(\gamma+i \delta)-V_{1}^{2}+\dot{V}_{1}\right]-i\left(1+\beta^{(0)}\right) V_{1}^{\prime} \in C_{\text {仲 }}^{m-7, e o}+i C_{\text {如 }}^{m-7, o o}$.

In the light of (M.7), (M.9) gives that $\dot{V}_{2}=-\left(b_{3}+i b_{4}\right) / 2$ and, because of the form of $b_{3}+i b_{4}$, compatibility conditions are not needed to solve for $Z_{2}$ :

$$
Z_{2}(\xi, \tau)=-\frac{1}{2} Z_{0}(\xi, \tau) \int_{0}^{\tau}\left(b_{3}+i b_{4}\right)(\xi, s) d s \in C_{\text {仲 }}^{m-7, e e}+i C_{\text {如 }}^{m-7, o e} .
$$

The coefficient of $\partial_{\tau}^{-2} \theta$ in the expression for $\mathcal{L} \vartheta-\kappa^{(0)} \vartheta$ is $\lambda_{0}+\lambda_{1} \mathcal{H}$ where

$$
\begin{aligned}
\lambda_{0}+i \lambda_{1}=\ddot{Z}_{2}- & i\left(1+\beta^{(0)}\right) Z_{2}^{\prime}-\left(\kappa^{(0)}+i \alpha\right) Z_{2} \\
& +2(\dot{\gamma}+i \dot{\delta}) Z_{2}-(\ddot{\gamma}+i \ddot{\delta}) Z_{1}+i \dot{\alpha} Z_{1} \in C_{\text {仲 }}^{m-8, e e}+i C_{\text {吅 }}^{m-8, o e} .
\end{aligned}
$$

We can show the following

Lemma M.3. The coefficients $\alpha_{j}, \beta_{j}, j=0,1,2$ and $\lambda_{0}, \lambda_{1}$ satisfy the following estimates

$$
\begin{aligned}
\left\|\alpha_{0}\right\|_{C^{s}}+\left\|\beta_{0}\right\|_{C^{s}}+\left\|\dot{\alpha}_{0}\right\|_{C^{s}}+\left\|\dot{\beta}_{0}\right\|_{C^{s}} & \leq c_{s}\left(M_{5}\right)\|w\|_{s+5} \\
\left\|\alpha_{1}\right\|_{C^{s}}+\left\|\beta_{1}\right\|_{C^{s}}+\left\|\dot{\alpha}_{1}\right\|_{C^{s}}+\left\|\dot{\beta}_{1}\right\|_{C^{s}} & \leq c_{s}\left(M_{5}\right)\|w\|_{s+6} \\
\left\|\alpha_{2}\right\|_{C^{s}}+\left\|\beta_{2}\right\|_{C^{s}}+\left\|\dot{\alpha}_{2}\right\|_{C^{s}}+\left\|\dot{\beta}_{2}\right\|_{C^{s}} & \leq c_{s}\left(M_{5}\right)\|w\|_{s+7} \\
\left\|\lambda_{0}\right\|_{C^{s}}+\left\|\lambda_{1}\right\|_{C^{s}} & \leq c_{s}\left(M_{5}\right)\|w\|_{s+8}
\end{aligned}
$$


Proof. From Remark 7.3, the estimate (7.9) for $\beta^{(0)}$, and the formula (7.19) for $\kappa^{(0)}$ we deduce that

$$
\left\|\rho_{0}-1\right\| C_{C^{s}}+\left\|\psi_{0}\right\|_{C^{s}} \leq c_{s}\left(M_{5}\right)\|w\|_{s+5},
$$

then the formula for $Z_{0}$ and (M.7) for $\dot{Z}_{0}$ lead to the first estimate in (M.14). Now the formula for $b_{1}+i b_{2}$ and (M.12) with (M.11) gives the second line of (M.14). Then the formulas for $b_{3}+i b_{4}$ and for $Z_{2}$, and (M.9) lead to the third line of (M.14). The formula for $\lambda_{0}+i \lambda_{1}$ then gives the last line of (M.14).

Finally, to derive the equation satisfied by $\vartheta$ we need to invert (M.1). Since all the coefficients in $\mathcal{P}^{-1}$ are in $C^{m-7}$, it follows from (7.16) that

$$
\mathcal{P}=\mathbb{I}+\sum_{n \geq 1}(-1)^{n}\left\{\alpha_{0}+\beta_{0} \mathcal{H}+\left(\alpha_{1}+\beta_{1} \mathcal{H}\right) \partial_{\tau}^{-1}+\left(\alpha_{2}+\beta_{2} \mathcal{H}\right) \partial_{\tau}^{-2}\right\}^{n}
$$

defines a bounded linear operator on $H^{s}$ for $s \leq m-7$ which is close to the identity for $\varepsilon$ small enough when $w=w_{\varepsilon}^{\left(N_{0}\right)}+\varepsilon^{N_{0}} u, N_{0} \geq 2$, since the coefficients are $O(\varepsilon)$. Let

$$
\begin{aligned}
& \mathcal{V}(\vartheta)=-\left\{1+\alpha_{0}+\beta_{0} \mathcal{H}+\left(\alpha_{1}+\beta_{1} \mathcal{H}\right) \partial_{\tau}^{-1}+\left(\alpha_{2}+\beta_{2} \mathcal{H}\right) \partial_{\tau}^{-2}\right\} \mathcal{G}_{0}(\mathcal{P} \vartheta) \\
&-\beta_{0}\left\{\mathcal{S}_{\gamma, \delta} \partial_{\tau}+\mathcal{S}_{0, \alpha}\right\} \mathcal{P} \vartheta-\beta_{2}\left\{\mathcal{S}_{\gamma, \delta} \partial_{\tau}^{-1}+\mathcal{S}_{0, \alpha} \partial_{\tau}^{-2}-2 \mathcal{S}_{\dot{\gamma}, \dot{\delta}} \partial_{\tau}^{-2}\right\} \mathcal{P} \vartheta \\
&-\beta_{1}\left\{\mathcal{S}_{\gamma, \delta}\left(1-\pi_{1}\right)-\mathcal{S}_{\dot{\gamma}, \dot{\delta}} \partial_{\tau}^{-1}+\mathcal{S}_{\ddot{\gamma}, \ddot{\delta}} \partial_{\tau}^{-2}+\mathcal{S}_{0, \alpha} \partial_{\tau}^{-1}-\mathcal{S}_{0, \dot{\alpha}} \partial_{\tau}^{-2}\right\} \mathcal{P} \vartheta \\
&-\left(1+\beta^{(0)}\right) \partial_{\xi}\left\{\mathcal{S}_{\alpha_{0}, \beta_{0}} \mathcal{P} \vartheta+\mathcal{S}_{\alpha_{1}, \beta_{1}} \partial_{\tau}^{-1} \mathcal{P} \vartheta+\mathcal{S}_{\alpha_{2}, \beta_{2}} \partial_{\tau}^{-2} \mathcal{P} \vartheta\right\} \\
&-\left\{2\left(\dot{\alpha}_{1}+\dot{\beta}_{1} \mathcal{H}\right)+\beta_{1} \delta-\alpha_{1} \gamma-\left(\beta_{1} \gamma+\alpha_{1} \delta\right) \mathcal{H}\right\} \pi_{1} \mathcal{P} \vartheta \\
&+\left(\alpha_{1}+\beta_{1} \mathcal{H}\right)\left\{3 \pi_{1}\left[(\gamma+\delta \mathcal{H})\left(1-\pi_{1}\right) \cdot\right]-\partial_{\tau}^{-1}\left[\ddot{\alpha} \mathcal{H} \partial_{\tau}^{-2} \cdot\right]+\right. \\
&\left.+2 \pi_{1}\left[\alpha \mathcal{H} \partial_{\tau}^{-1} \cdot\right]+\partial_{\tau}^{-1}\left[(\dddot{\gamma}+\dddot{\delta} \mathcal{H}) \partial_{\tau}^{-2} \cdot\right]-\partial_{\tau}^{-1} \alpha \mathcal{H} \pi_{1}\right\} \mathcal{P} \vartheta \\
&+\left(\alpha_{2}+\beta_{2} \mathcal{H}\right)\left\{3 \pi_{1}\left[(\gamma+\delta \mathcal{H}) \partial_{\tau}^{-1} \cdot\right]-3 \partial_{\tau}^{-1}\left[(\ddot{\gamma}+\ddot{\delta} \mathcal{H}) \partial_{\tau}^{-2} \cdot\right]+\right. \\
&+\partial_{\tau}^{-2}\left[(\dddot{\gamma}+\dddot{\delta} \mathcal{H}) \partial_{\tau}^{-2} \cdot+\pi_{1}\left[\alpha \mathcal{H} \partial_{\tau}^{-2} \cdot\right]-\partial_{\tau}^{-2} \alpha \mathcal{H} \pi_{1}+\right. \\
&\left.+2 \partial_{\tau}^{-1}\left[\dot{\alpha} \mathcal{H} \partial_{\tau}^{-2} \cdot\right]-\partial_{\tau}^{-2}\left[\ddot{\alpha} \mathcal{H} \partial_{\tau}^{-2} \cdot\right]\right\} \mathcal{P} \vartheta
\end{aligned}
$$

We have the following

Lemma M.4. The linear operator $\mathcal{V}$ can be decomposed as follows

$$
\mathcal{V}=\mathcal{V}_{1}+\mathcal{V}_{2}+\mathcal{V}_{3}
$$


where, for $\beta \leq 3$ and $0 \leq s+2 \beta \leq m-8$

$$
\left\|G_{\beta+2, \beta} \mathcal{V}_{1}(\vartheta)\right\|_{s} \leq c_{\beta, s}\left(M_{7}\right)\left(\|w\|_{s+2 \beta+8}\|\vartheta\|_{0}+\|w\|_{2 \beta+8}\|\vartheta\|_{s}\right),
$$

for $\beta \geq 3$ and $0 \leq s+3 \beta \leq m-5,(m \geq 14)$

$$
\left\|G_{\beta+2, \beta} \mathcal{V}_{1}(\vartheta)\right\|_{s} \leq c_{\beta, s}\left(M_{7}\right)\left(\|w\|_{s+3 \beta+5}\|\vartheta\|_{0}+\|w\|_{3 \beta+5}\|\vartheta\|_{s}\right),
$$

for any $\beta \geq 0$ and $0 \leq \beta+s \leq m-8$

$$
\left\|G_{0, \beta-1} \mathcal{V}_{2}(\vartheta)\right\| \|_{s} \leq c_{\beta, s}\left(M_{7}\right)\left(\|w\|_{s+\beta+8}\|\vartheta\|_{0}+\|w\|_{\beta+8}\|\vartheta\|_{s}\right),
$$

and for $0 \leq s \leq m-10$

$$
\left\|\partial_{\tau}^{3} \mathcal{V}_{3}(\vartheta)\right\|_{s} \leq c_{\beta, s}\left(M_{7}\right)\left(\|w\|_{s+10}\|\vartheta\|_{0}+\|w\|_{10}\|\vartheta\|_{s}\right) .
$$

Proof. First, from (M.14) and the formula for the operator $\mathcal{P}$,

$$
\|\mathcal{P} \vartheta\|_{s} \leq c_{s}\left(M_{4}\right)\left(\left(1+\|w\|_{7}\right)\|\vartheta\|_{s}+\|w\|_{s+7}\|\vartheta\|_{0}\right) .
$$

Consider now the first line $\mathcal{V}_{11}(\vartheta)$ of the above expression for $\mathcal{V}(\vartheta)$. This is a "product" of factors $C_{\natural \natural}^{m-7}$ (or their $\tau$-derivatives) and Q1D operators of order $(\beta+2, \beta)$. Operating with $\partial_{\tau}^{-(\beta+2)}$ transforms this (see Lemma M.2) into a sum of "products" of factors $C_{\sharp \mathrm{\natural}}^{m-8-\beta}$ and Q1D operators of orders $(0, \beta)$. Then applying $\partial_{\xi}^{\beta}$ leads to a sum of "products" of factors $r_{l} \in C_{t h}^{m-8-\beta-l}$ and Q1D operators $\mathcal{G}_{l} \circ \mathcal{P}$ of orders $(0, l)$. More precisely we have, recall from $(7.16)$ that $\theta=\mathcal{P} \vartheta$

$$
\begin{aligned}
\left\|G_{\beta+2, \beta} \mathcal{V}_{11}(\vartheta)\right\|_{s} & \leq c_{\beta, s} \sum_{l}\left(\left\|r_{l}\right\| C_{C^{s}}\left\|\mathcal{G}_{l}(\theta)\right\|_{0}+\left\|r_{l}\right\|_{C^{0}}\left\|\mathcal{G}_{l}(\theta)\right\|_{s}\right) \\
\left\|\mathcal{G}_{l}(\theta)\right\|_{s} & \leq c_{\beta, s}\left\|G_{\beta-l+2, \beta-l} \mathcal{G}_{0}(\theta)\right\|_{s} \\
\left\|r_{l}\right\|_{C^{s}} & \leq c_{s}\left(M_{5}\right)\left(1+\|w\|_{s+8+\beta+l}\right),
\end{aligned}
$$

which, because of Lemma 7.2 and standard interpolation inequalities, gives,

$$
\begin{array}{ll}
\left\|G_{\beta+2, \beta} \mathcal{V}_{11}(\vartheta)\right\|_{s} \leq c_{\beta, s}\left(M_{7}\right)\left(\|w\|_{s+2 \beta+8}\|\theta\|_{0}+\|w\|_{2 \beta+8}\|\theta\|_{s}\right), & \beta \leq 3, \\
\left\|G_{\beta+2, \beta} \mathcal{V}_{11}(\vartheta)\right\|_{s} \leq c_{\beta, s}\left(M_{7}\right)\left(\|w\|_{s+3 \beta+5}\|\theta\|_{0}+\|w\|_{3 \beta+5}\|\theta\|_{s}\right), & \beta \geq 3 .
\end{array}
$$

This, with (M.19) gives

$$
\begin{aligned}
& \left\|G_{\beta+2, \beta} \mathcal{V}_{11}(\vartheta)\right\|_{s} \leq c_{\beta, s}\left(M_{7}\right)\left(\|w\|_{s+2 \beta+8}\|\vartheta\|_{0}+\|w\|_{2 \beta+8}\|\vartheta\|_{s}\right), \quad \beta \leq 3 \\
& \left\|G_{\beta+2, \beta} \mathcal{V}_{11}(\vartheta)\right\|_{s} \leq c_{\beta, s}\left(M_{7}\right)\left(\|w\|_{s+3 \beta+5}\|\vartheta\|_{0}+\|w\|_{3 \beta+5}\|\vartheta\|_{s}\right), \quad \beta \geq 3
\end{aligned}
$$

Consider now the first term $\mathcal{V}_{12}(\vartheta)=-\beta_{0} \mathcal{S}_{\gamma, \delta} \partial_{\tau} \mathcal{P} \vartheta$ in the second line of the above expression for $\mathcal{V}(\vartheta)$, where $\beta_{0}$ satisfies (M.14), $\mathcal{P} \vartheta$ satisfies (M.19) and

$$
\mathcal{S}_{\gamma, \delta} \partial_{\tau}=\partial_{\tau} \mathcal{S}_{\gamma, \delta}-\mathcal{S}_{\dot{\gamma}, \dot{\delta}}
$$


satisfies, by Remarks 7.3 and M.1,

$$
\begin{aligned}
\left\|G_{1, \beta} \mathcal{S}_{\gamma, \delta} \partial_{\tau} \theta\right\|_{s} & \leq\left\|G_{0, \beta} \mathcal{S}_{\gamma, \delta} \theta\right\|_{s}+\left\|G_{0, \beta} \mathcal{S}_{\dot{\gamma}, \dot{\delta}} \theta\right\|_{s} \\
& \leq c_{\beta, s}\left(M_{4}\right)\left(\|w\|_{\beta+s+7}\|\theta\|_{0}+\|w\|_{\beta+7}\|\theta\|_{s}\right) .
\end{aligned}
$$

Similarly

$$
\left\|G_{1, \beta} \mathcal{V}_{12}(\vartheta)\right\| \|_{s} \leq c_{\beta, s}\left(M_{7}\right)\left(\|w\|_{\beta+s+6}\|\vartheta\|_{0}+\|w\|_{\beta+6}\|\vartheta\|_{s}\right) .
$$

With $\mathcal{V}_{1}(\vartheta)=\mathcal{V}_{11}(\vartheta)+\mathcal{V}_{12}(\vartheta)$ we obtain estimates (M.15) and (M.16) and $\mathcal{V}_{1}$ is of order $(\beta+2, \beta)$ on $H_{\text {如 }}^{s}, 0 \leq s+2 \beta \leq m-8$, if $\beta \leq 3$, or $0 \leq s+3 \beta \leq m-5$, if $\beta \geq 3(m \geq 14)$. (Recall that a Q1D operator of order $(1, \beta)$ is also of order $(\beta+2, \beta)$.) Now, in the above expression for $\mathcal{V}(\vartheta)$, let $\mathcal{V}_{21}(\vartheta)$ denote the second line except $\mathcal{V}_{12}(\vartheta)$, and the third line. This is a sum of "products" of factors $r_{l} \in C_{\text {b七 }}^{m-7}$ by Q1D operators $\mathcal{G}_{l} \circ \mathcal{P}$ of order $(0, \beta)$ with, by (M.4), Remark 7.3 and (M.14),

$$
\begin{aligned}
\left\|G_{0, \beta} \mathcal{G}_{l}(\theta)\right\|_{s} & \leq c_{\beta, s}\left(M_{4}\right)\left(\|w\|_{\beta+s+8}\|\theta\|_{0}+\|w\|_{\beta+8}\|\theta\| \|_{s}\right), \\
\left\|r_{l}\right\|_{C^{s}} & \leq c_{\beta, s}\left(M_{5}\right)\left(\|w\|_{s+7} .\right.
\end{aligned}
$$

Then, from (M.19) and standard interpolation,

$$
\left\|G_{0, \beta} \mathcal{V}_{21}(\vartheta)\right\|_{s} \leq c_{\beta, s}\left(M_{7}\right)\left(\|w\|_{\beta+s+8}\|\vartheta\|_{0}+\|w\|_{\beta+8}\|\vartheta\|_{s}\right) .
$$

The fourth line, denoted by $\mathcal{V}_{22}(\vartheta)$, of the expression for $\mathcal{V}(\vartheta)$, is estimated in the same way and the result is that

$$
\left\|G_{0, \beta-1} \mathcal{V}_{22}\right\|_{s} \leq c_{\beta, s}\left(M_{7}\right)\left(\|w\|_{\beta+s+8}\|\vartheta\|_{0}+\|w\|_{\beta+8}\|\vartheta\|_{s}\right) .
$$

Therefore $\mathcal{V}_{21}+\mathcal{V}_{22}=\mathcal{V}_{2}(\vartheta)$, defines a Q1D operator of order $(0, \beta-1)$ on $H_{\mathrm{ub}}^{s}$, $0 \leq \beta+s \leq m-8$ which satisfies the estimate (M.17). The last three lines of the above expression for $\mathcal{V}(\vartheta)$ give an operator $\mathcal{V}_{3}$ with the property that $\partial_{\tau}^{3} \mathcal{V}_{3}$ is bounded in $H_{4 h}^{s}, 0 \leq s \leq m-10$ and, from Remark 7.3, (M.14) and (M.19), we obtain the estimate (M.18) of the lemma.

The equation satisfied by $\vartheta$ is then of the form

$$
\partial_{\tau \tau} \vartheta-\left(1+\beta^{(0)}\right) \mathcal{H} \partial_{\xi} \vartheta-\kappa^{(0)} \vartheta=\left(\lambda_{0}+\lambda_{1} \mathcal{H}\right) \partial_{\tau}^{-2} \mathcal{P} \vartheta+\mathcal{V}(\vartheta)+h
$$

with

$$
h=\left\{1+\alpha_{0}+\beta_{0} \mathcal{H}+\left(\alpha_{1}+\beta_{1} \mathcal{H}\right) \partial_{\tau}^{-1}+\left(\alpha_{2}+\beta_{2} \mathcal{H}\right) \partial_{\tau}^{-2}\right\} g .
$$

Now suppose that $w=w_{\varepsilon}^{(N)}, N \geq 2$, is an approximate solution of $\mathcal{F}(w, 1+$ $\left.\varepsilon^{2} / 4\right)=0$. The coefficient $\kappa^{(0)}$ can be estimated as follows. Since

$$
\begin{aligned}
\kappa^{(0)} & =\frac{1}{16 \pi^{2}} \int_{-\pi}^{\pi} \int_{-\pi}^{\pi}\left\{\gamma^{2}-\delta^{2}\right\} d \tau d \xi \\
& =\frac{-1}{16 \pi^{2}} \int_{-\pi}^{\pi} \int_{-\pi}^{\pi}\left\{\frac{\left(1+\beta^{(0)}\right)^{2} e_{0}^{\prime 2}}{\left(1+d_{0}^{\prime}\right)^{2}}-\left(\frac{\ddot{e_{0}}}{\left(1+\dot{e_{0}}\right)^{2}}\right)^{2}\right\} \circ Q^{-1} d \xi d \tau
\end{aligned}
$$


we can use the Jacobian $\left(1+d_{0}^{\prime}\right)\left(1+\dot{e}_{0}\right)$ of the map $(y, t) \mapsto(\xi, \tau)$ to obtain

$$
\begin{aligned}
\kappa^{(0)} & =\frac{-1}{16 \pi^{2}} \int_{-\pi}^{\pi} \int_{-\pi}^{\pi}\left\{\frac{\left(1+\beta^{(0)}\right)^{2} e_{0}^{\prime 2}\left(1+\dot{e_{0}}\right)}{\left(1+d_{0}^{\prime}\right)}-\frac{\ddot{e}_{0}^{2}\left(1+d_{0}^{\prime}\right)}{\left(1+\dot{e}_{0}\right)^{3}}\right\} d y d t \\
& =\frac{-\left(1+\beta^{(0)}\right)^{2}}{16 \pi^{2}} \int_{-\pi}^{\pi} \int_{-\pi}^{\pi} \frac{\left(1+\dot{e_{0}}\right)}{\left(1+d_{0}^{\prime}\right)}\left\{e_{0}^{\prime 2}-\frac{\ddot{e}_{0}^{2}}{q^{2}}\right\} d y d t .
\end{aligned}
$$

It follows that $\kappa^{(0)}=O\left(\varepsilon^{4}\right)$ and, since $e_{0}^{\prime}=O\left(\varepsilon^{3}\right), \ddot{e_{0}}=\varepsilon^{2} \sin 2 t+O\left(\varepsilon^{3}\right)$, $q=1+O(\varepsilon)$, we obtain

$$
\kappa^{(0)}=\frac{\varepsilon^{4}}{8}+O\left(\varepsilon^{5}\right) .
$$

Then successively, we have (the time average of $-\gamma^{2}$ being $O\left(\varepsilon^{4}\right)$ )

$$
\begin{aligned}
\psi_{0}(\xi)= & O\left(\varepsilon^{3}\right), \\
\rho_{0}(\xi)= & \exp \left\{2 \varepsilon(\cos \xi-1)+\varepsilon^{2}(3 / 4-\cos 2 \xi)+O\left(\varepsilon^{3}\right)\right\} \\
b_{1}(\xi, \tau)= & \varepsilon^{2} \cos 2 \tau+O\left(\varepsilon^{3}\right), \\
b_{2}(\xi, \tau)= & O\left(\varepsilon^{3}\right), \\
\alpha_{0}(\xi, \tau)= & 2 \varepsilon(\cos \xi-1)+\varepsilon^{2}\left(4-\frac{1}{4} \cos 2 \tau-4 \cos \xi\right)+O\left(\varepsilon^{3}\right), \\
\beta_{0}(\xi, \tau)= & O\left(\varepsilon^{3}\right), \\
\alpha_{1}(\xi, \tau)= & -\frac{\varepsilon^{2}}{4} \sin 2 \tau+O\left(\varepsilon^{3}\right), \beta_{1}(\xi, \tau)=O\left(\varepsilon^{3}\right), \\
b_{3}(\xi, \tau)= & \varepsilon^{2} \sin 2 \tau+O\left(\varepsilon^{3}\right), b_{4}(\xi, \tau)=O\left(\varepsilon^{3}\right), \\
\alpha_{2}(\xi, \tau)= & -\frac{\varepsilon^{2}}{4}(1-\cos 2 \tau)+O\left(\varepsilon^{3}\right), \beta_{2}(\xi, \tau)=O\left(\varepsilon^{3}\right), \\
\lambda_{0}(\xi, \tau)= & -\varepsilon^{2} \cos 2 \tau+O\left(\varepsilon^{3}\right), \lambda_{1}(\xi, \tau)=O\left(\varepsilon^{3}\right), \\
& \mathcal{P} \vartheta=\vartheta+2 \varepsilon(1-\cos \xi) \vartheta+O\left(\varepsilon^{2}\|\vartheta\|\right) .
\end{aligned}
$$

Then, since

$$
\begin{aligned}
& \mathcal{S}_{\alpha_{1}} f=O\left(\varepsilon^{3}\|f\|\right), \mathcal{S}_{\alpha_{2}} f=O\left(\varepsilon^{3}\|f\|\right) \\
& \mathcal{S}_{\alpha_{0}} f=\left(2 \varepsilon-4 \varepsilon^{2}\right) \mathcal{S}_{\cos \xi} f+O\left(\varepsilon^{3}\|f\|\right)
\end{aligned}
$$

we have

$$
\begin{aligned}
\mathcal{V}(\vartheta)= & -\varepsilon \mathcal{G}_{01}(\vartheta)+2 \varepsilon^{2} \mathcal{G}_{01}(\cos \xi)-2 \varepsilon^{2}(\cos \xi) \mathcal{G}_{01}(\vartheta)+ \\
& -\varepsilon^{2} \mathcal{G}_{02}(\vartheta)-\left(2 \varepsilon-4 \varepsilon^{2}\right) \partial_{\xi} \mathcal{S}_{\cos \xi}(\mathcal{P} \vartheta)-2 \dot{\alpha}_{1} \pi_{1} \vartheta+O\left(\varepsilon^{3}\|\vartheta\|\right) .
\end{aligned}
$$

Hence

$$
\mathcal{V}=\varepsilon \mathcal{V}^{(1)}+\varepsilon^{2} \mathcal{V}^{(2)}+O\left(\varepsilon^{3}\right)
$$

with

$$
\mathcal{V}^{(1)}(\vartheta)=-2(\sin \tau \cos \xi) \pi_{0}\left(\partial_{\tau} \vartheta\right)
$$




$$
\begin{aligned}
\mathcal{V}^{(2)}(\vartheta)=(\cos 2 \tau) \pi_{1} \vartheta+2(\sin 2 \tau \cos \xi) \pi_{0}\left(\partial_{\tau} \vartheta\right. & \cos \xi) \\
& +\cos \xi(1+\cos 2 \tau) \pi_{0}(\vartheta \cos \xi) .
\end{aligned}
$$

From the above asymptotic expansions, we can infer the following estimates for the coefficients $\alpha_{j}, \beta_{j}, j=0,1,2$ and $\lambda_{0}, \lambda_{1}$, when $w=w_{\varepsilon}^{\left(N_{0}\right)}+\varepsilon^{N_{0}} u, \quad N_{0} \geq 2$ :

$$
\begin{array}{r}
\left\|\alpha_{0}\right\|_{C^{s}}+\left\|\beta_{0}\right\|_{C^{s}}+\left\|\dot{\alpha}_{0}\right\|_{C^{s}}+\left\|\dot{\beta}_{0}\right\|_{C^{s}} \leq \varepsilon c_{s}\left(M_{5}\right)\left(1+\|u\|_{s+5}\right), \\
\left\|\alpha_{1}\right\|_{C^{s}}+\left\|\beta_{1}\right\|_{C^{s}}+\left\|\dot{\alpha}_{1}\right\|_{C^{s}}+\left\|\dot{\beta}_{1}\right\|_{C^{s}} \leq \varepsilon^{2} c_{s}\left(M_{5}\right)\left(1+\|u\|_{s+6}\right), \\
\left\|\alpha_{2}\right\|_{C^{s}}+\left\|\beta_{2}\right\|_{C^{s}}+\left\|\dot{\alpha}_{2}\right\|_{C^{s}}+\left\|\dot{\beta}_{2}\right\|_{C^{s}} \leq \varepsilon^{2} c_{s}\left(M_{5}\right)\left(1+\|u\|_{s+7}\right), \\
\left\|\lambda_{0}\right\|_{C^{s}}+\left\|\lambda_{1}\right\|_{C^{s}} \leq \varepsilon^{2} c_{s}\left(M_{5}\right)\left(1+\|u\|_{s+8}\right) .
\end{array}
$$

The estimates for $\mathcal{V}_{j}$ follow directly from (M.15), (M.16), (M.17), (M.18) and the above asymptotic expansions. This ends the proof of the Theorem 7.5.

\section{N A Nash-Moser Theorem with a Parameter}

Let $\left(E_{s},\|\cdot\|_{s}\right)$ and $\left(F_{s},|\cdot|_{s}\right)$ denote two scales of Banach spaces parametrized by $s \in \mathbb{N}_{0}=\mathbb{N} \cup\{0\}$. Suppose that for $t<s$ there exist $c(t, s)$ such that

(i) $\|\cdot\|_{t} \leq c(t, s)\|\cdot\|_{s}, \quad|\cdot|_{t} \leq c(t, s)|\cdot|_{s}$.

(ii) For $\lambda \in[0,1]$ with $\lambda t+(1-\lambda) s \in \mathbb{N}$,

$$
\|\cdot\|_{\lambda t+(1-\lambda) s} \leq c(t, s)\|\cdot\|_{t}^{\lambda}\|\cdot\|_{s}^{1-\lambda}, \quad|\cdot|_{\lambda t+(1-\lambda) s} \leq c(t, s)|\cdot|_{t}^{\lambda}|\cdot|_{s}^{1-\lambda} .
$$

(iii) There exists a family of smoothing operators $S_{\wp}$ defined over the first scale such that for $\wp>0$ and $t<s$,

$$
\begin{gathered}
\left\|S_{\wp} u\right\|_{t} \leq c(t, s)\|u\|_{s}, \quad\left\|S_{\wp} u\right\|_{s} \leq c(t, s) \wp^{t-s}\|u\|_{t}, \\
\left\|S_{\wp} u-u\right\|_{t} \leq c(t, s) \wp^{s-t}\|u\|_{s},
\end{gathered}
$$

and, if $\varepsilon \mapsto \wp(\varepsilon)$ is a smooth, increasing, convex function on $[0, \infty)$ with $\wp(0)=0$, then, for $0<\varepsilon_{1}<\varepsilon_{2}$,

$$
\left\|\left(S_{\wp\left(\varepsilon_{1}\right)}-S_{\wp\left(\varepsilon_{2}\right)}\right) u\right\|_{s} \leq c(t, s)\left|\varepsilon_{1}-\varepsilon_{2}\right| \wp^{\prime}\left(\varepsilon_{2}\right) \wp\left(\varepsilon_{1}\right)^{t-s-1}\|u\|_{t} .
$$

Remark N.1. In the standing-wave problem the Banach spaces $E_{s}, F_{s}$ are closed subspaces of the Sobolev spaces $H_{\mathrm{u}}^{s, e e}$ (defined following (4.3)) and consist of functions of the form

$$
u=\sum_{\substack{m \neq n^{2} \\(m, n) \in \mathbb{Z}^{2}}} \hat{u}_{m n} e^{-i m \xi-i n \tau}
$$

with norm

$$
\|u\|_{s}^{2}=|u|_{s}^{2}=\sum_{n \in \mathbb{Z}^{2}}\left(m^{2}+n^{2}\right)^{s}\left|\hat{u}_{m n}\right|^{2}
$$


A smoothing operator with the required properties is defined by

$$
S_{\wp} u=\sum_{\substack{m \neq n^{2} \\(m, n) \in \mathbb{Z}^{2}}}(\wp|n|) \hat{u}_{m n} e^{-i m \xi-i n \tau},
$$

where $\varsigma: \mathbb{R}^{+} \mapsto \mathbb{R}^{+}$is a smooth function which equals 1 on $[0,1]$ and 0 on $[2, \infty)$.

Consider a family of operators $\Phi(\cdot, \varepsilon)$, depending on a small parameter $\varepsilon \in$ $\left[0, \varepsilon_{0}\right]$, which map a neighborhood of 0 in $E_{r}$ into $F_{\rho}$. Suppose that there exist

$$
\sigma \leq \rho \leq r-1, \quad \sigma, \rho, r \in \mathbb{N}_{0},
$$

and, for all $l \in \mathbb{N}_{0}$, numbers $c(l)>0$ and $\varepsilon(l) \in\left(0, \varepsilon_{0}\right]$ with the following properties for all $u, v, u_{i}, v_{i} \in B$ and $\varepsilon, \varepsilon_{i} \in\left[0, \varepsilon_{0}\right], i=1,2$, where $B=\{u \in$ $\left.E_{r}:\|u\|_{r} \leq R_{0}\right\}$ for some $R_{0}>0$ :

(A) The operator $\Phi: B \times\left[0, \varepsilon_{0}\right] \rightarrow F_{\rho}$ is twice continuously differentiable,

$$
|\Phi(u, \varepsilon)|_{\rho+l} \leq c(l)\left(1+\|u\|_{r+l}\right)
$$

and, for $u, v \in E_{r+l}, \varepsilon \in[0, \varepsilon(l)]$,

$$
\begin{aligned}
& |D(u, v, \varepsilon)|_{\rho+l} \\
& \leq c(l)\left(1+\|u\|_{r+l}+\|v\|_{r+l}\right)\|u-v\|_{r}^{2}+c(l)\|u-v\|_{r}\|u-v\|_{r+l},
\end{aligned}
$$

where

$$
D(u, v, \varepsilon)=\Phi(u, \varepsilon)-\Phi(v, \varepsilon)-\Phi_{u}^{\prime}(v, \varepsilon)(u-v) .
$$

Moreover,

$$
\begin{aligned}
& \left|D\left(u_{1}, v_{1}, \varepsilon_{1}\right)-D\left(u_{2}, v_{2}, \varepsilon_{2}\right)\right|_{\rho} \\
& \leq c\left(\left|\varepsilon_{1}-\varepsilon_{2}\right|+\left\|u_{1}-u_{2}\right\|_{r}+\left\|v_{1}-v_{2}\right\|_{r}\right)\left(\left\|u_{1}-v_{1}\right\|_{r}+\left\|u_{2}-v_{2}\right\|_{r}\right) .
\end{aligned}
$$

(B) There exists a family of bounded linear operators $\Lambda(u, \varepsilon): E_{r} \rightarrow F_{\rho}$, depending on $(u, \varepsilon) \in B \times\left[0, \varepsilon_{0}\right]$, with

$$
|\Lambda(u, \varepsilon) v|_{\rho} \leq c(0)\|v\|_{r}, \quad v \in E_{r},
$$

that approximates the Fréchet derivative $\Phi_{u}^{\prime}$ as follows. For $u \in E_{r+l} \cap B$, $\varepsilon \in[0, \varepsilon(l)]$ and $v \in E_{r+l}$,

$$
\begin{aligned}
\left|\Lambda(u, \varepsilon) v-\Phi_{u}^{\prime}(u, \varepsilon) v\right|_{\rho+l} \leq c(l)\left(1+\|u\|_{r+l}\right)|\Phi(u, \varepsilon)|_{r}\|v\|_{r} \\
+c(l)|\Phi(u, \varepsilon)|_{r+l}\|v\|_{r}+c(l)|\Phi(u, \varepsilon)|_{r}\|v\|_{r+l} .
\end{aligned}
$$


(C) When $u_{i} \in B \cap E_{r+l}, \varepsilon_{i} \in[0, \varepsilon(l)], i=1,2$,

$$
\begin{aligned}
& \left|\Phi\left(u_{1}, \varepsilon_{1}\right)-\Phi\left(u_{2}, \varepsilon_{2}\right)\right|_{\rho+l} \\
& \leq c(l)\left(1+\left\|u_{1}\right\|_{r+l}+\left\|u_{2}\right\|_{r+l}\right)\left(\left|\varepsilon_{1}-\varepsilon_{2}\right|+\left\|u_{1}-u_{2}\right\|_{r}\right) \\
& +c(l)\left\|u_{1}-u_{2}\right\|_{r+l}, \\
& \left|\left(\Phi_{u}^{\prime}\left(u_{1}, \varepsilon_{1}\right)-\Phi_{u}^{\prime}\left(u_{2}, \varepsilon_{2}\right)\right) v\right|_{\rho+l}+\left|\left(\Lambda\left(u_{1}, \varepsilon_{1}\right)-\Lambda\left(u_{2}, \varepsilon_{2}\right)\right) v\right|_{\rho+l} \leq \\
& c(l)\left(\left\|u_{1}-u_{2}\right\|_{r+l}+\left(\left|\varepsilon_{1}-\varepsilon_{2}\right|+\left\|u_{1}-u_{2}\right\|_{r}\right)\left(\left\|u_{1}\right\|_{r+l}+\left\|u_{2}\right\|_{r+l}\right)\right)\|v\|_{r} \\
& +\left(\left|\varepsilon_{1}-\varepsilon_{2}\right|+\left\|u_{1}-u_{2}\right\|_{r}\right)\|v\|_{r+l},
\end{aligned}
$$

(D) If a set $\mathcal{E} \subset[0, \varepsilon(l)]$ is dense at 0 and a mapping $\nu: \mathcal{E} \rightarrow B \cap E_{r+l}$ is Lipschitz in the sense that for $\varepsilon_{1}, \varepsilon_{2} \in \mathcal{E}$,

$$
\left\|\nu\left(\varepsilon_{1}\right)-\nu\left(\varepsilon_{2}\right)\right\|_{r} \leq C\left|\varepsilon_{1}-\varepsilon_{2}\right| \text { where } C=C(\nu), \text { constant, }
$$

then there is a set $\mathcal{E}(\nu) \subset \mathcal{E}$, which is also dense at 0 , such that, for any $\varepsilon \in \mathcal{E}(\nu)$ and $f \in F_{\rho+l}$, the equation $\Lambda(\nu(\varepsilon), \varepsilon) v=f$ has a unique solution satisfying

$$
\|v\|_{\rho-\sigma+l} \leq \varepsilon^{-\varrho} c(l)\left(|f|_{\rho+l}+\|\nu(\varepsilon)\|_{r+l}|f|_{\rho}\right) .
$$

(E) Suppose that $\nu_{0}: \mathcal{E}_{0} \rightarrow B \cap E_{r+l}$ and mappings $\nu_{k}: \cap_{i=0}^{k-1} \mathcal{E}\left(\nu_{i}\right) \rightarrow B \cap E_{r+l}$ satisfy, for a constant $C$ independent of $k \in \mathbb{N}$ sufficiently large,

$$
\begin{gathered}
\left\|\nu_{k}\left(\varepsilon_{1}\right)-\nu_{k}\left(\varepsilon_{2}\right)\right\|_{r} \leq C\left|\varepsilon_{1}-\varepsilon_{2}\right|, \quad \varepsilon_{1}, \varepsilon_{2} \in \cap_{j=0}^{k-1} \mathcal{E}\left(\nu_{j}\right), \\
\left\|\nu_{k+1}(\varepsilon)-\nu_{k}(\varepsilon)\right\|_{r} \leq \frac{1}{2^{k}}, \quad \varepsilon \in \cap_{j=0}^{k} \mathcal{E}\left(\nu_{j}\right) .
\end{gathered}
$$

Then $\cap_{j=0}^{\infty} \mathcal{E}\left(\nu_{j}\right)$ is dense at 0 , where the sets $\mathcal{E}\left(\nu_{j}\right)$ are defined in (D).

Theorem N.2. Suppose (A)-(E) hold and, for $N \in \mathbb{N}$ with $N \geq 2$, the equation

$$
\Phi(u, \varepsilon)=0, \quad \varepsilon \in\left[0, \varepsilon_{0}\right]
$$

has an approximate solution $u=u_{\varepsilon}^{(N)} \in \cap_{s \in \mathbb{N}_{0}} E_{s}$, with, for a constant $k(N, s)$,

$$
\left\|u_{\varepsilon}^{(N)}\right\|_{s} \leq k(N, s) \varepsilon, \quad\left|\Phi\left(\varepsilon, u_{\varepsilon}^{(N)}\right)\right|_{s} \leq k(N, s)|\varepsilon|^{N+1}
$$

and

$$
\left\|u_{\varepsilon_{1}}^{(N)}-u_{\varepsilon_{2}}^{(N)}\right\|_{s} \leq k(N, s)\left|\varepsilon_{1}-\varepsilon_{2}\right| .
$$

Then there is a set $\mathcal{E}$, which is dense at 0 , and a family

$$
\{u=\nu(\varepsilon): \varepsilon \in \mathcal{E}\} \subset E_{r}
$$

of solutions to (N.2) with $\left\|\nu\left(\varepsilon_{1}\right)-\nu\left(\varepsilon_{2}\right)\right\|_{r} \leq c\left|\varepsilon_{1}-\varepsilon_{2}\right|$ for some constant $c$. 
Proof. Let $d=r+\sigma-\rho \geq 1$ and fix integers $a \geq 2(r-\rho)+1$ and $b \geq d$ such that

$$
\begin{gathered}
b\left(\frac{1}{2}-\frac{d+r-\rho}{a}\right)>3 d+2 \varrho, \quad a>3(d+b+2 \varrho)+4, \\
\frac{2 \gamma}{3}-d \geq 6 d, \quad \frac{a}{3} \geq b+3 d+\varrho, \quad b \geq 12 d+\frac{3 \varrho}{2},
\end{gathered}
$$

where

$$
\gamma=b\left(1-\frac{r-\rho}{a}\right)-r+\rho .
$$

Choose $N \in \mathbb{N}$, a constant $R$ and $\varepsilon_{0} \in(0, \varepsilon(a+r)]\left(\varepsilon_{0}\right.$ smaller than before if necessary), such that the approximate solution $u_{\varepsilon}^{(N)}$ satisfies, for $\varepsilon, \varepsilon_{i} \in\left[0, \varepsilon_{0}\right]$,

$$
\begin{gathered}
\left\|u_{\varepsilon}^{(N)}\right\|_{r+a} \leq R / 2, \quad\left|\Phi\left(u_{\varepsilon}^{(N)}, \varepsilon\right)\right|_{\rho+a} \leq \varepsilon^{b+1+2 \varrho}, \\
\left\|u_{\varepsilon_{1}}^{(N)}-u_{\varepsilon_{2}}^{(N)}\right\|_{r+a} \leq 2^{-1} R\left|\varepsilon_{1}-\varepsilon_{2}\right| .
\end{gathered}
$$

Using (D) we now define sequences of $E_{r}$-valued functions, $\left\{\nu_{k}\right\},\left\{v_{k}\right\}$, and real-valued functions $\left\{\wp_{k}\right\}$, each function being defined on a subset of $\left[0, \varepsilon_{0}\right]$, as follows. Let $\wp_{0}(\varepsilon)=\varepsilon$ and $\nu_{0}(\varepsilon)=u_{\varepsilon}^{(N)}, \varepsilon \in \mathcal{E}:=\left[0, \varepsilon_{0}\right]$, and then, recursively,

$$
\begin{aligned}
\wp_{k+1}(\varepsilon) & =\wp_{k}(\varepsilon)^{3 / 2}=\varepsilon^{(3 / 2)^{k},} & & k \in \mathbb{N}_{0}, \\
\Lambda\left(\nu_{k}(\varepsilon), \varepsilon\right) v_{k}(\varepsilon) & =-\Phi\left(\nu_{k}(\varepsilon), \varepsilon\right), & & \varepsilon \in \cap_{j=0}^{k} \mathcal{E}\left(\nu_{j}\right), \\
\nu_{k+1}(\varepsilon) & =\nu_{k}(\varepsilon)+S_{\wp_{k+1}(\varepsilon)} v_{k}(\varepsilon), & & \varepsilon \in \cap_{j=0}^{k} \mathcal{E}\left(\nu_{j}\right) .
\end{aligned}
$$

The proof, that $\left\{\nu_{k}(\varepsilon)\right\}$ converges in $E_{r}$ to a solution $u=\nu(\varepsilon)$ of $\Phi(u, \varepsilon)=0$ for all $\varepsilon$ in a set which is dense at zero, needs estimates on this iteration process. For simplicity with notation we write $u_{k}^{i}, v_{k}^{i}, \Lambda_{k}^{i}, \Phi_{k}^{i}, S_{k}^{i}$ and $\Phi_{k}^{i \prime}$ instead of $\nu_{k}\left(\varepsilon_{i}\right), v_{k}\left(\varepsilon_{i}\right), \Lambda\left(\nu_{k}\left(\varepsilon_{i}\right), \varepsilon_{i}\right), \Phi\left(\nu_{k}\left(\varepsilon_{i}\right), \varepsilon_{i}\right), S_{\wp_{k}\left(\varepsilon_{i}\right)}$ and $\Phi_{u}^{\prime}\left(\nu_{k}\left(\varepsilon_{i}\right), \varepsilon_{i}\right)$, for $k \in \mathbb{N}$ and $i=1$, 2. In this notation, for $\varepsilon_{i} \in \mathcal{M}_{k}:=\cap_{j=0}^{k} \mathcal{E}\left(\nu_{j}\right) \subset\left[0, \varepsilon_{0}\right]$ and $i=1,2$,

$$
\Lambda_{k}^{i} v_{k}^{i}=-\Phi_{k}^{i}, \quad u_{k+1}^{i}=u_{k}^{i}+S_{k+1}^{i} v_{k}^{i} .
$$

The following lemmas deal with $\varepsilon_{1}, \varepsilon_{2} \in\left[0, \varepsilon_{0}\right]$ separately when $\varepsilon_{1} \leq \varepsilon_{2} / 2$ and $\varepsilon_{2} \geq \varepsilon_{1} \geq \varepsilon_{2} / 2$. The latter is the more difficult and we begin with it.

Lemma N.3. The interval $\left(0, \varepsilon_{0}\right]$ can be chosen, smaller if necessary but depending only on $R, a, b, r, \rho$ and $\sigma$, such that if $\varepsilon_{1}, \varepsilon_{2} \in \mathcal{M}_{k-1} \cap\left(0, \varepsilon_{0}\right]$ and $\varepsilon_{1} \in\left[\varepsilon_{2} / 2, \varepsilon_{2}\right]$ satisfy

$$
\begin{aligned}
\left\|u_{k-1}^{1}-u_{k-1}^{2}\right\|_{r} & \leq R\left|\varepsilon_{1}-\varepsilon_{2}\right|, \\
\left\|u_{k-1}^{1}-u_{k-1}^{2}\right\|_{r+a} & \leq R \wp_{k-1}\left(\varepsilon_{1}\right)^{-a}\left|\varepsilon_{1}-\varepsilon_{2}\right|, \\
\left\|u_{k-1}^{i}\right\|_{r} & \leq R \\
\left\|u_{k-1}^{i}\right\|_{r+a} & \leq \wp_{k-1}\left(\varepsilon_{i}\right)^{-a} \\
\left|\Phi_{k-1}^{i}\right|_{\rho} & \leq \wp_{k-1}\left(\varepsilon_{i}\right)^{b} \varepsilon_{i}^{\varrho \varrho+1} \leq 1
\end{aligned}
$$


for some $k \in \mathbb{N}$, then

$$
\begin{gathered}
\left\|\left(u_{k}^{1}-u_{k-1}^{1}\right)-\left(u_{k}^{2}-u_{k-1}^{2}\right)\right\|_{r} \leq \wp_{k}\left(\varepsilon_{2}\right)^{d}\left|\varepsilon_{1}-\varepsilon_{2}\right|, \\
\left\|\left(u_{k}^{1}-u_{k-1}^{1}\right)-\left(u_{k}^{2}-u_{k-1}^{2}\right)\right\|_{r+a} \leq \wp_{k}\left(\varepsilon_{2}\right)^{d-a}\left|\varepsilon_{1}-\varepsilon_{2}\right| .
\end{gathered}
$$

Proof. In what follows $c$ represents different constants, which depend only on $R, a, b, r, \rho$ and $\sigma$. We first observe that

$$
\begin{aligned}
\Phi_{k}^{1}-\Phi_{k}^{2}= & \left(\Lambda_{k-1}^{1}-\Lambda_{k-1}^{2}\right)\left(S_{k}^{2}-1\right) v_{k-1}^{2}+\Lambda_{k-1}^{1}\left(S_{k}^{2}-1\right)\left(v_{k-1}^{1}-v_{k-1}^{2}\right) \\
& +\left(\left(\Phi_{k-1}^{1}{ }^{\prime}-\Lambda_{k-1}^{1}\right)-\left(\Phi_{k-1}^{2}{ }^{\prime}-\Lambda_{k-1}^{2}\right)\right) S_{k}^{2} v_{k-1}^{2} \\
& \left.+\left(\Phi_{k-1}^{1}{ }^{\prime}-\Lambda_{k-1}^{1}\right)\right) S_{k}^{2}\left(v_{k-1}^{1}-v_{k-1}^{2}\right)+\Phi_{k-1}^{1}{ }^{\prime}\left(S_{k}^{1}-S_{k}^{2}\right) v_{k-1}^{1} \\
& +D\left(u_{k}^{1}, u_{k-1}^{1}, \varepsilon_{1}\right)-D\left(u_{k}^{2}, u_{k-1}^{2}, \varepsilon_{2}\right) .
\end{aligned}
$$

Hypotheses (N.10a), (N.10c), and (N.1g) with $l=0$, show that

$$
\left|\left(\Lambda_{k-1}^{1}-\Lambda_{k-1}^{2}\right)\left(S_{k}^{2}-1\right) v_{k-1}^{2}\right|_{\rho} \leq c\left|\varepsilon_{1}-\varepsilon_{2}\right|\left\|\left(S_{k}^{2}-1\right) v_{k-1}^{2}\right\|_{r}
$$

and (N.1d) implies that

$$
\left|\Lambda_{k-1}^{1}\left(S_{k}^{2}-1\right)\left(v_{k-1}^{1}-v_{k-1}^{2}\right)\right|_{\rho} \leq c\left\|\left(S_{k}^{2}-1\right)\left(v_{k-1}^{1}-v_{k-1}^{2}\right)\right\|_{r} .
$$

Now (N.1g) with $l=0$, and (N.10a) and (N.10c), yield

$$
\left|\left(\left(\Phi_{k-1}^{1}{ }^{\prime}-\Lambda_{k-1}^{1}\right)-\left(\Phi_{k-1}^{2}{ }^{\prime}-\Lambda_{k-1}^{2}\right)\right) S_{k}^{2} v_{k-1}^{2}\right|_{\rho} \leq c\left|\varepsilon_{1}-\varepsilon_{2}\right|\left\|S_{k}^{2} v_{k-1}^{2}\right\|_{r},
$$

and (N.1e) with $l=0$, with (N.10c), gives

$$
\left.\mid\left(\Phi_{k-1}^{1}{ }^{\prime}-\Lambda_{k-1}^{1}\right)\right)\left.S_{k}^{2}\left(v_{k-1}^{1}-v_{k-1}^{2}\right)\right|_{\rho} \leq c\left|\Phi_{k-1}^{1}\right|_{r}\left\|S_{k}^{2}\left(v_{k-1}^{2}-v_{k-1}^{1}\right)\right\|_{r} .
$$

By (A),

$$
\left|\Phi_{k-1}^{1}{ }^{\prime}\left(S_{k}^{1}-S_{k}^{2}\right) v_{k-1}^{1}\right|_{\rho} \leq c\left\|\left(S_{k}^{1}-S_{k}^{2}\right) v_{k-1}^{1}\right\|_{r}
$$

and (N.1c) and (N.9) imply that

$$
\begin{aligned}
& \left|D\left(u_{k}^{1}, u_{k-1}^{1}, \varepsilon_{1}\right)-D\left(u_{k}^{2}, u_{k-1}^{2}, \varepsilon_{2}\right)\right|_{\rho} \\
& \quad \leq c\left(\left|\varepsilon_{1}-\varepsilon_{2}\right|+\left\|u_{k-1}^{1}-u_{k-1}^{2}\right\|_{r}+\left\|u_{k}^{1}-u_{k}^{2}\right\|_{r}\right)\left(\left\|S_{k}^{1} v_{k-1}^{1}\right\|_{r}+\left\|S_{k}^{2} v_{k-1}^{2}\right\|_{r}\right) .
\end{aligned}
$$

Using the identity

$$
\begin{aligned}
u_{k}^{1}-u_{k}^{2} & =u_{k-1}^{1}-u_{k-1}^{2}+S_{k}^{1} v_{k-1}^{1}-S_{k}^{2} v_{k-1}^{2} \\
& =u_{k-1}^{1}-u_{k-1}^{2}+S_{k}^{2}\left(v_{k-1}^{1}-v_{k-1}^{2}\right)+\left(S_{k}^{1}-S_{k}^{2}\right) v_{k-1}^{1}
\end{aligned}
$$

and (N.10a), we obtain

$$
\begin{aligned}
\left|D\left(u_{k}^{1}, u_{k-1}^{1}, \varepsilon_{1}\right)-D\left(u_{k}^{2}, u_{k-1}^{2}, \varepsilon_{2}\right)\right|_{\rho} \leq & c\left|\varepsilon_{1}-\varepsilon_{2}\right| \sum_{i=1,2}\left\|S_{k}^{i} v_{k-1}^{i}\right\|_{r} \\
& +c\left\|S_{k}^{2}\left(v_{k-1}^{1}-v_{k-1}^{2}\right)\right\|_{r} \sum_{i=1,2}\left\|S_{k}^{i} v_{k-1}^{i}\right\|_{r} \\
& +c\left\|\left(S_{k}^{1}-S_{k}^{2}\right) v_{k-1}^{1}\right\|_{r} \sum_{i=1,2}\left\|S_{k}^{i} v_{k-1}^{i}\right\|_{r} .
\end{aligned}
$$


Therefore

$$
\left|\Phi_{k}^{1}-\Phi_{k}^{2}\right|_{\rho} \leq c \sum_{i=1}^{7} Q_{i}
$$

where

$$
\begin{aligned}
Q_{1} & =\left|\varepsilon_{1}-\varepsilon_{2}\right|\left\|\left(S_{k}^{2}-1\right) v_{k-1}^{2}\right\|_{r}, \\
Q_{2} & =\left\|\left(S_{k}^{2}-1\right)\left(v_{k-1}^{1}-v_{k-1}^{2}\right)\right\|_{r}, \\
Q_{3} & =\left|\varepsilon_{1}-\varepsilon_{2}\right|\left\|S_{k}^{2} v_{k-1}^{2}\right\|_{r}, \\
Q_{4} & =\left|\Phi_{k-1}^{1}\right|_{r}\left\|S_{k}^{2}\left(v_{k-1}^{2}-v_{k-1}^{1}\right)\right\|_{r}, \\
Q_{5} & =\left|\varepsilon_{1}-\varepsilon_{2}\right| \sum_{i=1,2}\left\|S_{k}^{i} v_{k-1}^{i}\right\|_{r}, \\
Q_{6} & =\left\|S_{k}^{2}\left(v_{k-1}^{1}-v_{k-1}^{2}\right)\right\|_{r} \sum_{i=1,2}\left\|S_{k}^{i} v_{k-1}^{i}\right\|_{r}, \\
Q_{7} & =\left\|\left(S_{k}^{1}-S_{k}^{2}\right) v_{k-1}^{1}\right\|_{r} \sum_{i=1,2}\left(\left\|S_{k}^{i} v_{k-1}^{i}\right\|_{r}+1\right) .
\end{aligned}
$$

Now we estimate the $Q_{j}$, one at a time. Recall that $d=r+\sigma-\rho$. It follows from (iii), the properties of smoothing operators, that

$$
\left\|\left(S_{k}^{2}-1\right) v_{k-1}^{2}\right\|_{r} \leq c \wp_{k}\left(\varepsilon_{2}\right)^{a-d}\left\|v_{k-1}^{2}\right\|_{\rho-\sigma+a} .
$$

Then, from estimate (N.1h) for solutions of equation $\Lambda_{k-1}^{2} v_{k-1}^{2}=-\Phi_{k-1}^{2}$, (N.1a) with $l=a$ and (N.10d), we find that

$$
\begin{aligned}
\left\|v_{k-1}^{2}\right\|_{\rho-\sigma+a} & \leq c \varepsilon_{2}^{-\varrho}\left(\left\|u_{k-1}^{2}\right\|_{r+a}\left|\Phi_{k-1}^{2}\right|_{\rho}+\left|\Phi_{k-1}^{2}\right|_{\rho+a}\right) \\
& \leq c \varepsilon_{2}^{-\varrho}\left(1+\left\|u_{k-1}^{2}\right\|_{r+a}\right) \leq c \varepsilon_{2}^{-\varrho} \wp_{k-1}\left(\varepsilon_{2}\right)^{-a} .
\end{aligned}
$$

Hence

$$
Q_{1} \leq c\left|\varepsilon_{1}-\varepsilon_{2}\right| \varepsilon_{2}^{-\varrho} \wp_{k}\left(\varepsilon_{2}\right)^{a-d} \wp_{k-1}\left(\varepsilon_{2}\right)^{-a} .
$$

Estimation of $Q_{2}$ is much more delicate. From (N.9),

$$
\Lambda_{k-1}^{1}\left(v_{k-1}^{1}-v_{k-1}^{2}\right)=\left(\Lambda_{k-1}^{2}-\Lambda_{k-1}^{1}\right) v_{k-1}^{2}+\left(\Phi_{k-1}^{2}-\Phi_{k-1}^{1}\right),
$$

and hence, by estimate (N.1h) from (D),

$$
\begin{aligned}
& \| v_{k-1}^{1}- v_{k-1}^{2} \|_{\rho-\sigma+a-d} \\
& \leq c \varepsilon_{1}^{-\varrho}\left\{\left(\left|\left(\Lambda_{k-1}^{1}-\Lambda_{k-1}^{2}\right) v_{k-1}^{2}\right|_{\rho+a-d}+\left|\Phi_{k-1}^{1}-\Phi_{k-1}^{2}\right|_{\rho+a-d}\right)\right. \\
&\left.+\left(\left|\left(\Lambda_{k-1}^{1}-\Lambda_{k-1}^{2}\right) v_{k-1}^{2}\right|_{\rho}+\left|\Phi_{k-1}^{1}-\Phi_{k-1}^{2}\right|_{\rho}\right)\left\|u_{k-1}^{1}\right\|_{r+a-d}\right\}
\end{aligned}
$$

From (N.10a), (N.10c), and (N.1g) with $l=0$, it follows since, by (A), $\Phi$ is continuously differentiable, that

$$
\begin{aligned}
\left|\Phi_{k-1}^{1}-\Phi_{k-1}^{2}\right|_{\rho} & \leq c\left|\varepsilon_{1}-\varepsilon_{2}\right| \\
\left|\left(\Lambda_{k-1}^{1}-\Lambda_{k-1}^{2}\right) v_{k-1}^{2}\right|_{\rho} & \leq c\left|\varepsilon_{1}-\varepsilon_{2}\right||| v_{k-1}^{2} \|_{r} .
\end{aligned}
$$


This shows that

$$
\begin{aligned}
& \left\|v_{k-1}^{1}-v_{k-1}^{2}\right\|_{\rho-\sigma+a-d} \\
& \leq c \varepsilon_{1}^{-\varrho}\left\{\left|\left(\Lambda_{k-1}^{1}-\Lambda_{k-1}^{2}\right) v_{k-1}^{2}\right|_{\rho+a-d}+\left|\Phi_{k-1}^{1}-\Phi_{k-1}^{2}\right|_{\rho+a-d}\right\} \\
& \quad+c \varepsilon_{1}^{-\varrho}\left|\varepsilon_{1}-\varepsilon_{2}\right|\left(1+\left\|v_{k-1}^{2}\right\|_{r}\right)\left\|u_{k-1}^{1}\right\|_{r+a-d} .
\end{aligned}
$$

From (N.1f) with $l=a,(\mathrm{~N} .10 \mathrm{a})$ and (N.10d),

$$
\left|\Phi_{k-1}^{1}-\Phi_{k-1}^{2}\right|_{\rho+a-d} \leq c\left|\Phi_{k-1}^{1}-\Phi_{k-1}^{2}\right|_{\rho+a} \leq c \wp_{k-1}\left(\varepsilon_{1}\right)^{-a}\left|\varepsilon_{1}-\varepsilon_{2}\right|,
$$

which, since $\left\|u_{k-1}^{1}\right\|_{r+a-d} \leq\left\|u_{k-1}^{1}\right\|_{r+a} \leq c \wp_{k-1}\left(\varepsilon_{1}\right)^{-a}$, by (i) and (N.10d), implies

$$
\begin{aligned}
\left\|v_{k-1}^{1}-v_{k-1}^{2}\right\|_{\rho-\sigma+a-d} \leq & c \varepsilon_{1}^{-\varrho}\left|\left(\Lambda_{k-1}^{1}-\Lambda_{k-1}^{2}\right) v_{k-1}^{2}\right|_{\rho+a-d} \\
& +c \varepsilon_{1}^{-\varrho} \wp_{k-1}\left(\varepsilon_{1}\right)^{-a}\left|\varepsilon_{1}-\varepsilon_{2}\right|\left\{1+\left\|v_{k-1}^{2}\right\|_{r}\right\} .
\end{aligned}
$$

Next, from the interpolation inequality in (ii), we obtain

$$
\left\|v_{k-1}^{2}\right\|_{r} \leq c\left\|v_{k-1}^{2}\right\|_{\rho-\sigma}^{1-d / a}\left\|v_{k-1}^{2}\right\|_{\rho-\sigma+a}^{d / a} .
$$

Since $\Lambda_{k-1}^{2} v_{k-1}^{2}=-\Phi_{k-1}^{2},(\mathrm{~N} .1 \mathrm{~h}),(\mathrm{N} .10 \mathrm{c}),(\mathrm{N} .10 \mathrm{e})$ and $l=a$ in (N.1a) yield

$$
\begin{aligned}
\left\|v_{k-1}^{2}\right\|_{r} & \leq c \varepsilon_{2}^{-\varrho}\left|\Phi_{k-1}^{2}\right|_{\rho}^{1-d / a}\left(\left|\Phi_{k-1}^{2}\right|_{\rho+a}+\left\|u_{k-1}^{2}\right\|_{r+a}\right)^{d / a} \\
& \leq c \varepsilon_{2}^{-\varrho}\left|\Phi_{k-1}^{2}\right|_{\rho}^{1-d / a}\left(1+\left\|u_{k-1}^{2}\right\|_{r+a}\right)^{d / a} .
\end{aligned}
$$

Therefore, by (N.10d) and (N.10e),

$$
\begin{aligned}
\left\|v_{k-1}^{2}\right\|_{r} & \leq c \varepsilon_{2}^{-\varrho}\left|\Phi_{k-1}^{2}\right|_{\rho}^{1-d / a} \wp_{k-1}\left(\varepsilon_{2}\right)^{-d} \\
& \leq c \varepsilon_{2}^{\varrho+1-d(2 \rho+1) / a} \wp_{k-1}\left(\varepsilon_{2}\right)^{b} \wp_{k-1}\left(\varepsilon_{2}\right)^{-d(1+b / a)} \leq c,
\end{aligned}
$$

because the first inequality in (N.5) implies that $b \geq d(1+b / a)$, and the second implies that $a \geq 2 d \geq d(2 \rho+1) /(\rho+1)$. Now (N.16) and (N.17) imply that

$$
\begin{aligned}
\left\|v_{k-1}^{1}-v_{k-1}^{2}\right\|_{\rho-\sigma+a-d} \leq c \varepsilon_{1}^{-\varrho} \mid\left(\Lambda_{k-1}^{1}-\right. & \left.\Lambda_{k-1}^{2}\right)\left.v_{k-1}^{2}\right|_{\rho+a-d} \\
& +c \varepsilon_{1}^{-\varrho} \wp_{k-1}\left(\varepsilon_{1}\right)^{-a}\left|\varepsilon_{1}-\varepsilon_{2}\right| .
\end{aligned}
$$

Also (N.1g) with $l=a-d$ and (N.10a) imply that

$$
\begin{aligned}
& \left|\left(\Lambda_{k-1}^{1}-\Lambda_{k-1}^{2}\right) v_{k-1}^{2}\right|_{\rho+a-d} \\
& \quad \leq c\left(\left\|u_{k-1}^{1}-u_{k-1}^{2}\right\|_{r+a-d}+\left|\varepsilon_{1}-\varepsilon_{2}\right|\left(\left\|u_{1}\right\|_{r+a-d}+\left\|u_{2}\right\|_{r+a-d}\right)\right)\left\|v_{k-1}^{2}\right\|_{r} \\
& +c\left|\varepsilon_{1}-\varepsilon_{2}\right|\left\|v_{k-1}^{2}\right\|_{r+a-d} .
\end{aligned}
$$

Therefore, from (N.17), (N.10b) and (N.10d), it follows that

$$
\left|\left(\Lambda_{k-1}^{1}-\Lambda_{k-1}^{2}\right) v_{k-1}^{2}\right|_{\rho+a-d} \leq c\left|\varepsilon_{1}-\varepsilon_{2}\right|\left(\wp_{k-1}\left(\varepsilon_{1}\right)^{-a}+\left\|v_{k-1}^{2}\right\|_{r+a-d}\right) .
$$


Since $r+a-d=\rho-\sigma+a$ and $\Lambda_{k-1}^{2} v_{k-1}^{2}=-\Phi_{k-1}^{2}$, we also have

$$
\begin{aligned}
\left\|v_{k-1}^{2}\right\|_{r+a-d} & \leq c \varepsilon_{2}^{-\varrho}\left(\left|\Phi_{k-1}^{2}\right|_{\rho+a}+\left\|u_{k-1}^{2}\right\|_{r+a}\left|\Phi_{k-1}^{2}\right|_{\rho}\right) \\
& \leq c \varepsilon_{2}^{-\varrho}\left(1+\left\|u_{k-1}^{2}\right\|_{r+a}\right) \leq c \varepsilon_{2}^{-} \varrho_{\wp_{k-1}}\left(\varepsilon_{2}\right)^{-a} .
\end{aligned}
$$

This, with (N.18) and the fact that $\varepsilon_{1}<\varepsilon_{2}$, gives

$$
\left\|v_{k-1}^{1}-v_{k-1}^{2}\right\|_{\rho-\sigma+a-d} \leq c \varepsilon_{1}^{-2 \varrho}\left|\varepsilon_{1}-\varepsilon_{2}\right| \wp_{k-1}\left(\varepsilon_{1}\right)^{-a} .
$$

Since $d=r-\rho+\sigma$, the expression for $Q_{2}$ yields the estimate

$$
\begin{aligned}
Q_{2} & \leq c \wp_{k}\left(\varepsilon_{2}\right)^{a-2 d}\left\|v_{k-1}^{1}-v_{k-1}^{2}\right\|_{\rho-\sigma+a-d} \\
& \leq c \varepsilon_{1}^{-2 \varrho}\left|\varepsilon_{1}-\varepsilon_{2}\right| \wp_{k}\left(\varepsilon_{2}\right)^{a-2 d} \wp_{k-1}\left(\varepsilon_{1}\right)^{-a} .
\end{aligned}
$$

To estimate $Q_{3}$, note from (iii), (N.10c) and (N.1h), that

$$
\left\|S_{k}^{2} v_{k-1}^{2}\right\|_{r} \leq c \wp_{k}\left(\varepsilon_{2}\right)^{-d}\left\|v_{k-1}^{2}\right\|_{\rho-\sigma} \leq c \varepsilon_{2}^{-\varrho} \wp_{k}\left(\varepsilon_{2}\right)^{-d}\left|\Phi_{k-1}^{2}\right|_{\rho}
$$

and hence, from (N.10e), that

$$
Q_{3} \leq c \varepsilon_{2}^{\varrho+1}\left|\varepsilon_{1}-\varepsilon_{2}\right| \wp_{k-1}\left(\varepsilon_{2}\right)^{b} \wp_{k}\left(\varepsilon_{2}\right)^{-d} .
$$

To estimate $Q_{4}$, note that

$$
\left\|S_{k}^{2}\left(v_{k-1}^{2}-v_{k-1}^{1}\right)\right\|_{r} \leq c \wp_{k}\left(\varepsilon_{2}\right)^{-d}\left\|v_{k-1}^{2}-v_{k-1}^{1}\right\|_{\rho-\sigma} .
$$

Therefore an appeal to (N.1h), for solutions of equation (N.15) with $l=0$, (N.10c) and (N.17), followed by an appeal to (N.1g) with $l=0$ and (N.10c), yields

$$
\begin{array}{r}
\left\|S_{k}^{2}\left(v_{k-1}^{2}-v_{k-1}^{1}\right)\right\|_{r} \\
\leq c \wp_{k}\left(\varepsilon_{2}\right)^{-d} \varepsilon_{1}^{-\varrho}\left\{\left|\left(\Lambda_{k-1}^{2}-\Lambda_{k-1}^{1}\right) v_{k-1}^{2}\right|_{\rho}+\left|\Phi_{k-1}^{1}-\Phi_{k-1}^{2}\right|_{\rho}\right\} \\
\leq c \varepsilon_{1}^{-\varrho} \wp_{k}\left(\varepsilon_{2}\right)^{-d}\left\{\left|\varepsilon_{1}-\varepsilon_{2}\right|\left\|v_{k-1}^{2}\right\|_{r}+\left|\Phi_{k-1}^{1}-\Phi_{k-1}^{2}\right|_{\rho}\right\} .
\end{array}
$$

But (N.17) and (N.1f) with $l=0$ implies that

$$
\left|\Phi_{k-1}^{1}-\Phi_{k-1}^{2}\right|_{\rho} \leq c\left|\varepsilon_{1}-\varepsilon_{2}\right|+c|| u_{k-1}^{1}-u_{k-2}^{2} \|_{r} \leq c\left|\varepsilon_{1}-\varepsilon_{2}\right|,
$$

and hence

$$
\left\|S_{k}^{2}\left(v_{k-1}^{2}-v_{k-1}^{1}\right)\right\|_{r} \leq c \varepsilon_{1}^{-\varrho} \wp_{k}\left(\varepsilon_{2}\right)^{-d}\left|\varepsilon_{1}-\varepsilon_{2}\right| .
$$

On the other hand, from the interpolation inequality (iii) and (N.1a),

$$
\left|\Phi_{k-1}^{1}\right|_{r} \leq c\left|\Phi_{k-1}^{1}\right|_{\rho}^{1-\frac{r-\rho}{a}}\left|\Phi_{k-1}^{1}\right|_{\rho+a}^{\frac{r-\rho}{\rho}} \leq c\left|\Phi_{k-1}^{1}\right|_{\rho}^{1-\frac{r-\rho}{a}}\left(1+\left\|u_{k-1}^{1}\right\|_{r+a}\right)^{\frac{r-\rho}{a}},
$$

which, because of (N.10d) and (N.10e), gives

$$
\begin{aligned}
\left|\Phi_{k-1}^{1}\right|_{r} & \leq c \wp_{k-1}\left(\varepsilon_{1}\right)^{-r+\rho}\left|\Phi_{k-1}^{1}\right|_{\rho}^{1-\frac{r-\rho}{a}} \\
& \leq c \varepsilon_{1}^{(2 \varrho+1)\left(1-\frac{r-\rho}{a}\right)} \wp_{k-1}\left(\varepsilon_{1}\right)^{b\left(1-\frac{r-\rho}{a}\right)-r+\rho} .
\end{aligned}
$$


Combining this with (N.23) and the fact that $\varepsilon_{1}^{(2 \varrho+1)\left(1-\frac{r-\rho}{a}\right)-\varrho} \leq 1$, we arrive at the estimate

$$
Q_{4} \leq c\left|\varepsilon_{1}-\varepsilon_{2}\right| \wp_{k-1}\left(\varepsilon_{1}\right)^{\gamma} \wp_{k}\left(\varepsilon_{2}\right)^{-d} \text { where } \gamma=b\left(1-\frac{r-\rho}{a}\right)-r+\rho .
$$

For $Q_{5}$ and $Q_{6}$, note, from (iii), (N.9) and (N.1h), (N.10e) and (N.6), that

$$
\begin{aligned}
\left\|S_{k}^{i} v_{k-1}^{i}\right\|_{r} & \leq c \wp_{k}\left(\varepsilon_{i}\right)^{-d}\left\|v_{k-1}^{i}\right\|_{\rho-\sigma} \leq c \varepsilon_{i}^{-\varrho} \wp_{k}\left(\varepsilon_{i}\right)^{-d}\left|\Phi_{k-1}^{i}\right|_{\rho}, \\
& \leq c \wp_{k-1}\left(\varepsilon_{i}\right)^{-3 d / 2} \wp_{k-1}\left(\varepsilon_{i}\right)^{b} \varepsilon_{i}^{\varrho+1} \leq c .
\end{aligned}
$$

Therefore

$$
Q_{5} \leq c\left|\varepsilon_{1}-\varepsilon_{2}\right| \sum_{i=1,2} \wp_{k}\left(\varepsilon_{i}\right)^{-d} \wp_{k-1}\left(\varepsilon_{i}\right)^{b} .
$$

Similarly (N.23) and (N.26) imply that

$$
Q_{6} \leq c \varepsilon_{1}^{-\varrho}\left|\varepsilon_{1}-\varepsilon_{2}\right| \wp_{k}\left(\varepsilon_{2}\right)^{-d} \sum_{i=1,2} \wp_{k}\left(\varepsilon_{i}\right)^{-d} \wp_{k-1}\left(\varepsilon_{i}\right)^{b} .
$$

Finally, from (N.26), the last paragraph of (iii) and (N.10e),

$$
\begin{aligned}
Q_{7} & \leq c\left\|\left(S_{k}^{1}-S_{k}^{2}\right) v_{k-1}^{1}\right\|_{r} \\
& \leq c\left|\varepsilon_{1}-\varepsilon_{2}\right| \wp_{k}^{\prime}\left(\varepsilon_{2}\right) \wp_{k}\left(\varepsilon_{1}\right)^{-d-1}\left\|v_{k-1}^{1}\right\|_{\rho-\sigma} \\
& \leq c \varepsilon_{1}^{-\varrho}\left|\varepsilon_{1}-\varepsilon_{2}\right| \wp_{k}^{\prime}\left(\varepsilon_{2}\right) \wp_{k}\left(\varepsilon_{1}\right)^{-d-1}\left|\Phi_{k-1}^{1}\right|_{\rho} \\
& \leq c \varepsilon_{1}^{\varrho+1}\left|\varepsilon_{1}-\varepsilon_{2}\right| \wp_{k}^{\prime}\left(\varepsilon_{2}\right) \wp_{k}\left(\varepsilon_{1}\right)^{b-d-1} .
\end{aligned}
$$

Since $\wp_{k}^{\prime}\left(\varepsilon_{2}\right)=\varepsilon_{2}^{-1}(3 / 2)^{k} \wp_{k}\left(\varepsilon_{2}\right)$ and $\varepsilon_{1} \leq \varepsilon_{2}$, we obtain

$$
Q_{7} \leq c \varepsilon_{1}^{\varrho}\left|\varepsilon_{1}-\varepsilon_{2}\right| \wp_{k}\left(\varepsilon_{1}\right)^{b-d-1}(3 / 2)^{k} \wp_{k}\left(\varepsilon_{2}\right) .
$$

We now collect the estimates (N.14), (N.19), (N.21), (N.25), (N.27), (N.28) and (N.29) for the quantities $Q_{i}$. Recall that $\varepsilon_{1} \in\left[\varepsilon_{2} / 2, \varepsilon_{2}\right] \subset\left[0, \varepsilon_{0}\right]$. Then $\varepsilon_{0}>0$ can be chosen sufficiently small that

$$
\begin{gathered}
Q_{i} \leq c\left|\varepsilon_{1}-\varepsilon_{2}\right| q_{i}, \quad i=1, \ldots, 7, \\
\text { where, since } \wp_{k+1}\left(\varepsilon_{i}\right)=\wp_{k}\left(\varepsilon_{i}\right)^{3 / 2}=\varepsilon_{i}^{(3 / 2)^{k}} \text { and } 2 b>3 d, \\
q_{1}=\varepsilon_{2}^{-\varrho} \wp_{k}\left(\varepsilon_{2}\right)^{a-d} \wp_{k-1}\left(\varepsilon_{2}\right)^{-a} \leq c \wp_{k}\left(\varepsilon_{2}\right)^{a-d-\varrho} \wp_{k-1}\left(\varepsilon_{2}\right)^{-a}, \\
q_{2}=\varepsilon_{1}^{-2 \varrho} \wp_{k}\left(\varepsilon_{2}\right)^{a-2 d} \wp_{k-1}\left(\varepsilon_{1}\right)^{-a} \leq c \wp_{k}\left(\varepsilon_{2}\right)^{a-3 d-\varrho} \wp_{k-1}\left(\varepsilon_{2}\right)^{-a}, \\
q_{3}=\varepsilon_{2}^{\varrho+1} \wp_{k-1}\left(\varepsilon_{2}\right)^{b} \wp_{k}\left(\varepsilon_{2}\right)^{-d} \leq c \wp_{k-1}\left(\varepsilon_{2}\right)^{b} \wp_{k}\left(\varepsilon_{2}\right)^{-d}, \\
q_{4}=\wp_{k-1}\left(\varepsilon_{1}\right)^{\gamma} \wp_{k}\left(\varepsilon_{2}\right)^{-d} \leq \wp_{k-1}\left(\varepsilon_{2}\right)^{\gamma} \wp_{k}\left(\varepsilon_{2}\right)^{-d}, \\
q_{5}=\sum_{i=1,2} \wp_{k}\left(\varepsilon_{i}\right)^{-d} \wp_{k-1}\left(\varepsilon_{i}\right)^{b} \leq c \wp_{k}\left(\varepsilon_{2}\right)^{-d} \wp_{k-1}\left(\varepsilon_{2}\right)^{b}, \\
q_{6}=c \varepsilon_{1}^{-\varrho} \wp_{k}\left(\varepsilon_{2}\right)^{-d} \sum_{i=1,2} \wp_{k}\left(\varepsilon_{i}\right)^{-d} \wp_{k-1}\left(\varepsilon_{i}\right)^{b} \leq c \wp_{k}\left(\varepsilon_{2}\right)^{-2 d-\varrho} \wp_{k-1}\left(\varepsilon_{2}\right)^{b}, \\
q_{7}=\varepsilon_{1}^{\varrho} \wp_{k}\left(\varepsilon_{1}\right)^{b-d-1}(3 / 2)^{k} \wp_{k}\left(\varepsilon_{2}\right) \leq c \wp_{k}\left(\varepsilon_{2}\right)^{b-d}(3 / 2)^{k} \leq c \wp_{k-1}\left(\varepsilon_{2}\right)^{b-d} .
\end{gathered}
$$


Therefore, from (N.13) and the definition of $\gamma$,

$$
\begin{aligned}
\left|\Phi_{k}^{1}-\Phi_{k}^{2}\right|_{\rho} \leq & c \sum_{i=1}^{7} Q_{i} \leq c\left|\varepsilon_{1}-\varepsilon_{2}\right|\left(\wp_{k}\left(\varepsilon_{2}\right)^{a-3 d-\varrho} \wp_{k-1}\left(\varepsilon_{2}\right)^{-a}\right. \\
& \left.\wp_{k}\left(\varepsilon_{2}\right)^{-d} \wp_{k-1}\left(\varepsilon_{2}\right)^{\gamma}+\wp_{k}\left(\varepsilon_{2}\right)^{-2 d-\varrho} \wp_{k-1}\left(\varepsilon_{2}\right)^{b}\right) \\
= & c\left|\varepsilon_{1}-\varepsilon_{2}\right|\left(\wp_{k}\left(\varepsilon_{2}\right)^{\frac{a}{3}-3 d-\varrho}+\wp_{k}\left(\varepsilon_{2}\right)^{\frac{2 \gamma}{3}-d}+\wp_{k}\left(\varepsilon_{2}\right)^{\frac{2 b}{3}-2 d-\varrho}\right) \\
\leq & c\left|\varepsilon_{1}-\varepsilon_{2}\right|\left(\wp_{k}\left(\varepsilon_{2}\right)^{\frac{a}{3}-3 d-\varrho}+\wp_{k}\left(\varepsilon_{2}\right)^{\frac{2 b}{3}-2 d-\varrho}\right) .
\end{aligned}
$$

Since, by (N.6),

$$
\frac{2 \gamma}{3}-d \geq 6 d, \quad \frac{a}{3}-3 d-\varrho \geq b \geq 6 d, \quad \frac{2 b}{3}-2 d \geq 6 d+\varrho
$$

we conclude that $\varepsilon_{0}>0$ can be chosen such that

$$
\left|\Phi_{k}^{1}-\Phi_{k}^{2}\right|_{\varrho} \leq c_{1}\left|\varepsilon_{1}-\varepsilon_{2}\right|_{\wp}\left(\varepsilon_{2}\right)^{6 d},
$$

where $c_{1}$ depends only on $R, a, b, r, \varrho, \rho$ and $\sigma$. Recall, from (N.9), that

$$
\begin{aligned}
\left(u_{k}^{1}-u_{k-1}^{1}\right)-\left(u_{k}^{2}-u_{k-1}^{2}\right) & =S_{k}^{1} v_{k-1}^{1}-S_{k}^{2} v_{k-1}^{2} \\
& =S_{k}^{2}\left(v_{k-1}^{1}-v_{k-1}^{2}\right)+\left(S_{k}^{1}-S_{k}^{2}\right) v_{k-1}^{1} .
\end{aligned}
$$

Let $\iota \in\{0,1\}$. Then property (iii) of smoothing operators, with (N.17), (N.22) and (N.30), yields

$$
\begin{aligned}
& \left\|S_{k}^{2}\left(v_{k-1}^{2}-v_{k-1}^{1}\right)\right\|_{r+\iota a} \leq c \wp_{k}\left(\varepsilon_{2}\right)^{-d-\iota a}\left\|v_{k-1}^{2}-v_{k-1}^{1}\right\|_{\rho-\sigma} \\
& \leq c \wp_{k}\left(\varepsilon_{2}\right)^{-d-\iota a} \varepsilon_{1}^{-\varrho}\left\{\left|\left(\Lambda_{k-1}^{2}-\Lambda_{k-1}^{1}\right) v_{k-1}^{2}\right|_{\rho}+\left|\Phi_{k-1}^{1}-\Phi_{k-1}^{2}\right|_{\rho}\right\} \\
& \leq c \wp_{k}\left(\varepsilon_{2}\right)^{-d-\iota a} \varepsilon_{1}^{-\varrho}\left\{\left|\varepsilon_{1}-\varepsilon_{2}\right|\left\|v_{k-1}^{2}\right\|_{r}+\left|\Phi_{k-1}^{1}-\Phi_{k-1}^{2}\right|_{\rho}\right\} \\
& \leq c\left|\varepsilon_{1}-\varepsilon_{2}\right| \wp_{k}\left(\varepsilon_{2}\right)^{-\iota a-d} \varepsilon_{1}^{-\varrho}\left\{\left\|v_{k-1}^{2}\right\|_{r}+c_{1} \wp_{k}\left(\varepsilon_{2}\right)^{6 d}\right\} \\
& \leq c\left|\varepsilon_{1}-\varepsilon_{2}\right| \wp_{k}\left(\varepsilon_{2}\right)^{-\iota a-d} \varepsilon_{1}^{-\varrho}\left\{\varepsilon_{2}^{\varrho+1-d(2 \varrho+1) / a} \wp_{k-1}\left(\varepsilon_{2}\right)^{b-d(1+b) / a}+\wp_{k}\left(\varepsilon_{2}\right)^{6 d}\right\} .
\end{aligned}
$$

Since $\varrho+1-d(2 \varrho+1) / a>0$, taking $\iota \in\{0,1\}$ gives, when $\varepsilon_{0}>0$ has been chosen sufficiently small,

$$
\begin{aligned}
\left\|S_{k}^{2}\left(v_{k-1}^{2}-v_{k-1}^{1}\right)\right\|_{r} & \leq c\left|\varepsilon_{1}-\varepsilon_{2}\right|\left(\wp_{k}\left(\varepsilon_{2}\right)^{\frac{2 b}{3}-2 d}+\wp_{k}\left(\varepsilon_{2}\right)^{4 d}\right), \\
\left\|S_{k}^{2}\left(v_{k-1}^{2}-v_{k-1}^{1}\right)\right\|_{r+a} & \leq c\left|\varepsilon_{1}-\varepsilon_{2}\right| \wp_{k}\left(\varepsilon_{2}\right)^{-a}\left(\wp_{k}\left(\varepsilon_{2}\right)^{\frac{2 b}{3}-2 d}+\wp_{k}\left(\varepsilon_{2}\right)^{4 d}\right) .
\end{aligned}
$$

Also, from the last part of (iii), (N.1h), (N.10c) and (N.10e), we have

$$
\begin{aligned}
\left\|\left(S_{k}^{1}-S_{k}^{2}\right) v_{k-1}^{1}\right\|_{r+\iota a} & \leq c\left|\varepsilon_{1}-\varepsilon_{2}\right| \wp_{k}^{\prime}\left(\varepsilon_{2}\right) \wp_{k}\left(\varepsilon_{1}\right)^{-\iota a-d-1}\left\|v_{k-1}^{1}\right\|_{\rho-\sigma} \\
& \leq c \varepsilon_{1}^{-\varrho}\left|\varepsilon_{1}-\varepsilon_{2}\right| \wp_{k}^{\prime}\left(\varepsilon_{2}\right) \wp_{k}\left(\varepsilon_{1}\right)^{-\iota a-d-1}\left|\Phi_{k-1}^{1}\right|_{\rho} \\
& \leq c \varepsilon_{1}^{\varrho+1}\left|\varepsilon_{1}-\varepsilon_{2}\right| \wp_{k}^{\prime}\left(\varepsilon_{2}\right) \wp_{k-1}\left(\varepsilon_{1}\right)^{b} \wp_{k}\left(\varepsilon_{1}\right)^{-\iota a-d-1} .
\end{aligned}
$$


Since $\wp_{k}^{\prime}\left(\varepsilon_{2}\right)=\varepsilon_{2}^{-1}(3 / 2)^{k} \wp_{k}\left(\varepsilon_{2}\right)$ we obtain, for $\iota \in\{0,1\}$,

$$
\begin{aligned}
\left\|\left(S_{k}^{1}-S_{k}^{2}\right) v_{k-1}^{1}\right\|_{r+\iota a} & \leq c \varepsilon_{1}^{\varrho}\left|\varepsilon_{1}-\varepsilon_{2}\right| \wp_{k}\left(\varepsilon_{1}\right)^{\frac{2}{3} b-d-1}(3 / 2)^{k} \wp_{k}\left(\varepsilon_{2}\right) \wp_{k}\left(\varepsilon_{1}\right)^{-\iota a} \\
& \leq c\left|\varepsilon_{1}-\varepsilon_{2}\right| \wp_{k}\left(\varepsilon_{2}\right)^{\frac{2}{3} b-d}(3 / 2)^{k} \wp_{k}\left(\varepsilon_{1}\right)^{-\iota a} .
\end{aligned}
$$

Next note that for sufficiently small $\varepsilon<2^{-a / d}$, independent of $k$,

$$
(3 / 2)^{k} \wp_{k}\left(\varepsilon_{1}\right)^{-\iota a} \leq(3 / 2)^{k} 2^{a\left(\frac{3}{2}\right)^{k}} \wp_{k}\left(\varepsilon_{2}\right)^{-\iota a} \leq c \wp_{k}\left(\varepsilon_{2}\right)^{-\iota a-d} .
$$

Therefore

$$
\begin{aligned}
\left\|\left(S_{k}^{1}-S_{k}^{2}\right) v_{k-1}^{1}\right\|_{r+\iota a} & \leq c\left|\varepsilon_{1}-\varepsilon_{2}\right| \wp_{k}\left(\varepsilon_{2}\right)^{\frac{2}{3} b-2 d} \wp_{k}\left(\varepsilon_{2}\right)^{-\iota a} \\
& \leq c\left|\varepsilon_{1}-\varepsilon_{2}\right| \wp_{k}\left(\varepsilon_{2}\right)^{4 d} \wp_{k}\left(\varepsilon_{2}\right)^{-\iota a}
\end{aligned}
$$

Substituting (N.32), (N.33), (N.34) into (N.31) leads to (N.11), (N.12), and the lemma follows from a smaller choice of $\varepsilon_{0}>0$ if necessary.

Lemma N.4. The interval $\left(0, \varepsilon_{0}\right]$ can be chosen, smaller if necessary but depending only on $R, a, b, r, \rho$ and $\sigma$, such that if $\varepsilon_{1}, \varepsilon_{2} \in \mathcal{M}_{k-1} \cap\left(0, \varepsilon_{0}\right]$ with $\varepsilon_{1} \in\left(0, \varepsilon_{2} / 2\right]$,

$$
\left\|u_{k-1}^{i}\right\|_{r} \leq R \text { and }\left|\Phi_{k-1}^{i}\right|_{\rho} \leq \wp_{k-1}\left(\varepsilon_{i}\right)^{b} \varepsilon_{i}^{2 \varrho+1} \leq 1 .
$$

Then

$$
\left\|\left(u_{k}^{1}-u_{k-1}^{1}\right)-\left(u_{k}^{2}-u_{k-1}^{2}\right)\right\|_{r} \leq \wp_{k}(\varepsilon)^{d}\left|\varepsilon_{1}-\varepsilon_{2}\right| .
$$

Proof. By (N.9), (iii) and (N.1h)

$$
\begin{aligned}
\|\left(u_{k}^{1}-u_{k-1}^{1}\right) & -\left(u_{k}^{2}-u_{k-1}^{2}\right) \|_{r} \\
& =\left\|S_{k}^{1} v_{k-1}^{1}-S_{k}^{2} v_{k-1}^{2}\right\|_{r} \\
& \left.\leq\left\|S_{k}^{1} v_{k-1}^{1}\right\|_{r}+\| S_{k}^{2} v_{k-1}^{2}\right) \|_{r} \\
& \leq c\left(\wp_{k}\left(\varepsilon_{1}\right)^{-d} \varepsilon_{1}^{-\varrho}\left|\Phi_{k-1}^{1}\right|_{\rho}+\wp_{k}\left(\varepsilon_{2}\right)^{-d} \varepsilon_{2}^{-\varrho}\left|\Phi_{k-1}^{2}\right|_{\rho}\right) \\
& \leq c \sum_{i=1,2} \varepsilon_{i}^{\varrho+1} \wp_{k}\left(\varepsilon_{i}\right)^{-d} \wp_{k-1}\left(\varepsilon_{i}\right)^{b} \leq c \varepsilon_{2} \wp_{k}\left(\varepsilon_{2}\right)^{\frac{2 b}{3}-d} .
\end{aligned}
$$

It remains to note that

$$
\varepsilon_{2} \leq 2\left|\varepsilon_{1}-\varepsilon_{2}\right|, \quad \wp_{k}\left(\varepsilon_{2}\right)^{\frac{2 b}{3}-d} \leq c \wp_{k}\left(\varepsilon_{2}\right)^{4 d},
$$

and the lemma follows.

Lemma N.5. Let $R$ be chosen as in (N.7). In (N.8) suppose that $v_{k-1}(\varepsilon)$, $\nu_{k}(\varepsilon)$ and $\Phi_{k}(\varepsilon):=\Phi\left(\nu_{k}(\varepsilon), \varepsilon\right)$ are well defined for $\varepsilon \in \mathcal{M}_{k}, 0 \leq k \leq m$, with $\left|\Phi_{0}(\varepsilon)\right|_{\rho} \leq \varepsilon^{b+2 \varrho+1}$ and, for the constant $R$ in (N.7),

$$
\left\|\nu_{k}(\varepsilon)\right\|_{r} \leq R, \quad\left\|\nu_{k-1}(\varepsilon)\right\|_{r+a} \leq \wp_{k-1}(\varepsilon)^{-a}, \quad\left|\Phi_{k-1}(\varepsilon)\right|_{\rho} \leq 1 .
$$

Then $\varepsilon_{0}>0$ can be chosen, independently of $m$, such that

$$
\left|\Phi_{k}(\varepsilon)\right|_{\rho} \leq \wp_{k}(\varepsilon)^{b} \varepsilon^{2 \varrho+1} \leq 1 \text { for all } 0 \leq k \leq m \text { and } \varepsilon \in \mathcal{M}_{m-1} .
$$


Proof. In what follows $c$ denotes various constants which are independent of $k$ and $\varepsilon$ but depend on $R, a, b, d, \rho, \sigma$ and $r$. Let $\Phi_{k}, \Lambda_{k}, \wp_{k}, u_{k}, v_{k}$ denote $\Phi_{k}(\epsilon), \Lambda_{k}\left(\nu_{k}(\varepsilon), \varepsilon\right), \wp_{k}(\varepsilon), \nu_{k}(\varepsilon), v_{k}(\varepsilon)$. Then by (D), $v_{k-1}$ exists for all $\varepsilon \in$ $\left(0, \varepsilon_{0}\right] \cap \mathcal{M}_{k-1}$ and

$$
\Phi_{k}=\Lambda_{k-1}\left(S_{\wp_{k}}-1\right) v_{k-1}+\left(\Phi_{k-1}^{\prime}-\Lambda_{k-1}\right)\left(u_{k}-u_{k-1}\right)+D\left(u_{k}, u_{k-1}, \varepsilon\right) .
$$

It follows from (iii), and (A) and (B) with $l=0$, that $\left|\Phi_{k}\right|_{\rho} \leq c\left(I_{1}+I_{2}+I_{3}\right)$ where

$$
I_{1}=\wp_{k}^{a-d}\left\|v_{k-1}\right\|_{\rho+a-\sigma}, \quad I_{2}=\left|\Phi_{k-1}\right|_{r}\left\|u_{k}-u_{k-1}\right\|_{r}, \quad I_{3}=\left\|u_{k}-u_{k-1}\right\|_{r}^{2} .
$$

Estimating $v_{k-1}$ from (D), $\left|\Phi_{k-1}\right|_{\rho+a}$ from (A) and $\left|\Phi_{k-1}\right|_{\rho}$ from the hypotheses of the Lemma, yields, for $\varepsilon \in(0, \varepsilon(a)] \cap \mathcal{M}_{k-1}$,

$$
I_{1} \leq c \varepsilon^{-\varrho} \wp_{k}^{a-d}\left(\left\|u_{k-1}\right\|_{r+a}+\left|\Phi_{k-1}\right|_{\rho+a}\right) \leq c \varepsilon^{-\varrho} \wp_{k}^{a-d} \wp_{k-1}^{-a} .
$$

It follows from the interpolation inequalities (ii) that

$$
\left\|u_{k}-u_{k-1}\right\|_{r} \leq c\left\|u_{k}-u_{k-1}\right\|_{\rho-\sigma}^{\alpha}\left\|u_{k}-u_{k-1}\right\|_{a+\rho-\sigma}^{1-\alpha} \text { where } \alpha=1-d / a .
$$

Also from (iii), (D) and (N.8c) it follows that

$$
\left\|u_{k}-u_{k-1}\right\|_{\rho-\sigma} \leq c\left\|v_{k-1}\right\|_{\rho-\sigma} \leq c \varepsilon^{-\varrho}\left|\Phi_{k-1}\right|_{\rho}, \quad \varepsilon \in(0, \varepsilon(0)] \cap \mathcal{M}_{k-1}
$$

and similarly for $\varepsilon \in(0, \varepsilon(a)] \cap \mathcal{M}_{k-1}$,

$$
\begin{aligned}
\left\|u_{k}-u_{k-1}\right\|_{\rho-\sigma+a} & \leq c\left\|v_{k-1}\right\|_{\rho-\sigma+a} \\
& \leq c \varepsilon^{-\varrho}\left(\left\|u_{k-1}\right\|_{r+a}+\left|\Phi_{k-1}\right|_{\rho+a}\right) \leq c \varepsilon^{-\varrho} \wp_{k-1}^{-a} .
\end{aligned}
$$

Moreover, the interpolation inequality (iii) gives

$$
\left|\Phi_{k-1}\right|_{r} \leq c\left|\Phi_{k-1}\right|_{\rho}^{1-\frac{r-\rho}{a}}\left|\Phi_{k-1}\right|_{\rho+a}^{\frac{r-\rho}{a}} .
$$

Therefore, for $\varepsilon \in(0, \varepsilon(a)] \cap \mathcal{M}_{k-1}$,

$$
\begin{aligned}
I_{2} & =\left|\Phi_{k-1}\right|_{r}\left\|u_{k}-u_{k-1}\right\|_{r} \\
& \leq c\left|\Phi_{k-1}\right|_{r}\left\|u_{k}-u_{k-1}\right\|_{\rho-\sigma}^{\alpha}\left\|u_{k}-u_{k-1}\right\|_{a+\rho-\sigma}^{1-\alpha} \\
& \leq \frac{c}{\varepsilon^{\varrho}}\left|\Phi_{k-1}\right|_{r}\left|\Phi_{k-1}\right|_{\rho}^{\alpha} \wp_{k-1}^{-a(1-\alpha)} \\
& =\frac{c}{\varepsilon^{\varrho}}\left|\Phi_{k-1}\right|_{r}\left|\Phi_{k-1}\right|_{\rho}^{1-(d / a)} \wp_{k-1}^{-d} \\
& \leq \frac{c}{\varepsilon^{\varrho}}\left|\Phi_{k-1}\right|_{\rho}^{2-\frac{d+r-\rho}{a}} \wp_{k-1}^{\sigma-2 d},
\end{aligned}
$$

where we have used (A) and the present hypothesis. Now (D) and (iii) imply that

$$
\left\|u_{k}-u_{k-1}\right\|_{r} \leq c \wp_{k}^{-d}\left\|v_{k-1}\right\|_{\rho-\sigma} \leq \frac{c}{\varepsilon^{\varrho} \wp_{k}^{d}}\left|\Phi_{k-1}\right|_{\rho}, \quad \varepsilon \in(0, \varepsilon(0)] \cap \mathcal{M}_{k-1} .
$$


Hence, for all $k \leq m$ and for $\varepsilon \in\left(0, \varepsilon^{\prime}\right] \cap \mathcal{M}_{k-1}, \varepsilon^{\prime}=\min \{\varepsilon(0), \varepsilon(a)\}$,

$$
\begin{aligned}
I_{1} & +I_{2}+I_{3} \leq \frac{c}{\varepsilon^{\varrho}}\left\{\wp_{k}^{a-d} \wp_{k-1}^{-a}+\left|\Phi_{k-1}\right|_{\rho}^{2-\frac{d+r-\rho}{a}} \wp_{k-1}^{\sigma-2 d}+\frac{1}{\wp_{k}^{2 d} \varepsilon^{\varrho}}\left|\Phi_{k-1}\right|_{\rho}^{2}\right\} \\
& =\frac{c}{\varepsilon^{\varrho}}\left\{\wp_{k}^{a-d} \wp_{k-1}^{-a}+\left|\Phi_{k-1}\right|_{\rho}^{2-\frac{d+r-\rho}{a}} \wp_{k}^{\frac{-2(2 d-\sigma)}{3}}+\frac{1}{\wp_{k}^{2 d} \varepsilon^{\varrho}}\left|\Phi_{k-1}\right|_{\rho}^{2}\right\} \\
& \leq \frac{c}{\varepsilon^{\varrho}}\left(\wp_{k}^{a-d} \wp_{k-1}^{-a}+\frac{\wp_{k}^{-2 d}}{\varepsilon^{\varrho}}\left|\Phi_{k-1}\right|_{\rho}^{2-\frac{d+r-\rho}{a}}\right),
\end{aligned}
$$

where $c$ and $\varepsilon^{\prime}$ do not depend on $k$. Let $\delta_{k}=\left|\Phi_{k}\right|_{\rho} \wp_{k}^{-b}$. Then, by (N.36),

$$
\begin{aligned}
\delta_{k} & =\wp_{k}^{-b}\left|\Phi_{k}\right|_{\rho} \leq c \wp_{k}^{-b}\left(I_{1}+I_{2}+I_{3}\right) \\
& \leq \frac{c}{\varepsilon^{\varrho}}\left\{\wp_{k}^{a-b-d} \wp_{k-1}^{-a}+\frac{\wp_{k}^{-2 d-b}}{\varepsilon^{\varrho}}\left|\Phi_{k-1}\right|_{\rho}^{2-\frac{d+r-\rho}{a}}\right\} \\
& =\frac{c}{\varepsilon^{\varrho}}\left\{\wp_{k-1}^{\frac{a}{2}-\frac{3}{2}(b+d)}+\frac{1}{\varepsilon^{\varrho}} \delta_{k-1}^{2-\frac{d+r-\rho}{a}} \wp_{k-1}^{b\left(\frac{1}{2}-\frac{d+r-\rho}{a}\right)-3 d}\right\} .
\end{aligned}
$$

Now the choice of $a$ and $b$ means that

$$
\frac{a}{2}-\frac{3}{2}(b+d)>3 \varrho+2, \quad 2-\frac{d+r-\rho}{a}>\frac{5}{3}, \quad b\left(\frac{1}{2}-\frac{d+r-\rho}{a}\right)-3 d>2 \varrho .
$$

and the fact that $\wp_{k-1} \leq \varepsilon$ then gives that

$$
\delta_{k} \leq \frac{c}{\varepsilon^{\varrho}}\left\{\wp_{k-1}^{3 \varrho+2}+\varepsilon^{\varrho} \delta_{k-1}^{5 / 3}\right\}, \quad 0 \leq k \leq m .
$$

For $k=0$ the inequality (N.35) is true by hypothesis, since $\wp_{0}(\varepsilon)=\varepsilon$. Now suppose (N.35) holds for $0 \leq k=j-1 \leq m-1$. From (N.37) it follows that

$$
\delta_{j} \leq \frac{c}{\varepsilon^{\varrho}}\left\{\varepsilon^{3 \varrho+2}+\varepsilon^{(10 \varrho+5) / 3}\right\} \leq \varepsilon^{2 \varrho+1}
$$

for all non-zero $\varepsilon \in \mathcal{M}_{m-1}$ sufficiently small (independent of $j$ ). The lemma follows by induction.

Proof of Theorem N.2 concluded. We now show that $\varepsilon_{0}>0$ can be chosen, smaller if necessary, such that, for all $k \in \mathbb{N}$ and $\varepsilon_{i}, \varepsilon \in\left(0, \varepsilon_{0}\right] \cap \mathcal{M}_{k-1}$,

$$
\begin{gathered}
\left\|\nu_{k}(\varepsilon)\right\|_{r} \leq R, \quad\left\|\nu_{k}(\varepsilon)\right\|_{r+a} \leq \wp_{k}(\varepsilon)^{-a}, \quad\left|\Phi_{k}(\varepsilon)\right|_{\rho} \leq \wp_{k}(\varepsilon)^{b} \varepsilon^{2 \rho+1} \leq 1, \\
\left\|\nu_{k}\left(\varepsilon_{1}\right)-\nu_{k}\left(\varepsilon_{2}\right)\right\|_{r} \leq R\left|\varepsilon_{1}-\varepsilon_{2}\right|, \quad \varepsilon_{2} / 2 \leq \varepsilon_{1} \leq \varepsilon_{2} .
\end{gathered}
$$

Here $R$ is the constant in (N.7) where it was observed that for $k=0$ these inequalities (in fact with $R / 2$ instead of $R$ ) follow from hypotheses (N.3) and (N.4) of Theorem N.2. Suppose inequalities (N.38) hold for every $k, 0 \leq k \leq m$. Then, by (N.7) and Lemma N.5, $\varepsilon_{0}>0$ can be chosen so that for all $\varepsilon \in\left(0, \varepsilon_{0}\right]$,

$$
\left|\Phi_{k}(\varepsilon)\right|_{\rho} \leq \varepsilon^{2 \varrho+1} \wp_{k}(\varepsilon)^{b} \leq 1, \quad 0 \leq k \leq m, \quad \varepsilon \in \mathcal{M}_{m-1} .
$$


To estimate $\left\|u_{m+1}\right\|_{r+\iota a}, \iota \in\{0,1\}$, note, from (iii) and (N.1h) with $l=0$ that, for $0 \leq m^{\prime} \leq m$ and $\varepsilon \in \mathcal{M}_{m} \subset \mathcal{M}_{m-1}$,

$$
\begin{aligned}
\left\|\nu_{m+1}(\varepsilon)-\nu_{m^{\prime}}(\varepsilon)\right\|_{r+\iota a} & \leq \sum_{k=m^{\prime}}^{m}\left\|\nu_{k+1}(\varepsilon)-\nu_{k}(\varepsilon)\right\|_{r+\iota a} \\
& =\sum_{k=m^{\prime}}^{m}\left\|S_{\wp_{k+1}(\varepsilon)^{\prime}} v_{k}(\varepsilon)\right\|_{r+\iota a} \\
& \leq c \sum_{k=m^{\prime}}^{m} \wp_{k+1}(\varepsilon)^{-d-\iota a}\left\|v_{k}(\varepsilon)\right\|_{\rho-\sigma} \\
& \leq \frac{c}{\varepsilon^{\varrho}} \sum_{k=m^{\prime}}^{m} \wp_{k+1}(\varepsilon)^{-d-\iota a}\left|\Phi_{k}(\varepsilon)\right|_{\rho} \\
& \leq \frac{c}{\varepsilon^{\varrho}} \sum_{k=m^{\prime}}^{m} \wp_{k+1}(\varepsilon)^{-d-\iota a} \wp_{k}(\varepsilon)^{b} \varepsilon^{2 \varrho+1}, \quad \text { by }(\mathrm{N} .40), \\
& =c \varepsilon^{\varrho+1} \wp_{m+1}(\varepsilon)^{-\iota a} \sum_{k=m^{\prime}}^{m} \wp_{m}(\varepsilon)^{\frac{3}{2} \iota a} \wp_{k}(\varepsilon)^{-\frac{3}{2}(d+\iota a)+b} \\
& \leq c \varepsilon^{\varrho+1} \wp_{m+1}(\varepsilon)^{-\iota a} \sum_{k=m^{\prime}}^{m} \wp_{k}(\varepsilon)^{b-3 d / 2} \\
& \leq c \varepsilon^{\varrho+1} \wp_{m+1}(\varepsilon)^{-\iota a} .
\end{aligned}
$$

Hence $\iota=0$ gives

$$
\left\|\nu_{m+1}(\varepsilon)\right\|_{r} \leq\left\|\nu_{0}(\varepsilon)\right\|_{r}+c \varepsilon^{\varrho+1} \leq R
$$

for all non-zero $\varepsilon \in \mathcal{M}_{m}$ sufficiently small. Similarly, $\iota=1$ gives

$$
\left\|\nu_{m+1}(\varepsilon)\right\|_{r+a} \leq c_{0}\left\|\nu_{0}\right\|_{r+a}+c \varepsilon^{\varrho+1} \wp_{m+1}(\varepsilon)^{-a} \leq \wp_{m+1}(\varepsilon)^{-a}
$$

for all non zero $\varepsilon \in \mathcal{M}_{m}$ sufficiently small. Therefore, by Lemma N.5, for $\varepsilon \in$ $\mathcal{M}_{m}$ sufficiently small independent of $k,\left|\Phi_{k}(\varepsilon)\right| \leq \wp_{k}(\varepsilon)^{b} \varepsilon^{2 \varrho+1} \leq 1$ for $k \leq m+1$. Hence (N.38) holds for all $k$, and $\varepsilon \in \mathcal{M}_{k-1}$ with $\varepsilon<\varepsilon_{0}$, for some $\varepsilon_{0}$ sufficiently small, independent of $k$. Next we show that $\varepsilon_{0}>0$ can be chosen so that (N.39) holds. First suppose that this is true for $\varepsilon_{1}, \varepsilon_{2} \in \mathcal{M}_{k-1}$ when $\varepsilon_{2} / 2 \leq \varepsilon_{1} \leq \varepsilon_{2}$ for $k$ with $0 \leq k \leq m$. Then by Lemma N.3,

$$
\left\|\left(u_{k}^{1}-u_{k-1}^{1}\right)-\left(u_{k}^{2}-u_{k-1}^{2}\right)\right\|_{r} \leq \wp_{k}\left(\varepsilon_{2}\right)^{d}\left|\varepsilon_{1}-\varepsilon_{2}\right| .
$$


for all $k \leq m+1$ and $\varepsilon_{i} \in\left(0, \varepsilon_{0}\right] \cap \mathcal{M}_{m}$. Thus

$$
\begin{aligned}
\left\|u_{m+1}^{1}-u_{m+1}^{2}\right\|_{r} & \leq \sum_{k=0}^{m}\left\|\left(u_{k+1}^{1}-u_{k+1}^{2}\right)-\left(u_{k}^{1}-u_{k}^{2}\right)\right\|_{r}+\left|\varepsilon_{1}-\varepsilon_{2}\right| R / 2 \\
& \leq\left|\varepsilon_{1}-\varepsilon_{2}\right|\left\{c_{1} \sum_{k=0}^{m} \wp_{k+1}\left(\varepsilon_{2}\right)^{d}+R / 2\right\} \\
& \leq\left|\varepsilon_{1}-\varepsilon_{2}\right|\left(c \wp_{1}\left(\varepsilon_{2}\right)+R / 2\right) \\
& \leq R\left|\varepsilon_{1}-\varepsilon_{2}\right|,
\end{aligned}
$$

which implies (N.39) for all $k$ when $\varepsilon_{0}>0$ is sufficiently small and $\varepsilon_{1}, \varepsilon_{2} \in$ $\mathcal{M}_{k-1}$ with $\varepsilon_{2} / 2 \leq \varepsilon_{1} \leq \varepsilon_{2}$. Now suppose that $\varepsilon_{1} \in\left(0, \varepsilon_{2} / 2\right]$. A repeat of the preceding argument, using Lemma N.5, yields (N.39) for all $k$ when $\varepsilon_{1}, \varepsilon_{2} \in$ $\cap \mathcal{M}_{k-1}$ in this case also. Therefore (N.39) holds for all $\varepsilon_{1}, \varepsilon_{2} \in \cap \mathcal{M}_{k-1}$. It remains to note that

$$
\left\|\nu_{k}(\varepsilon)-\nu_{k-1}(\varepsilon)\right\|_{r} \leq c_{1 \wp_{k}}(\varepsilon) \leq 1 / 2^{k},
$$

and, hence the set $\cap_{k} \mathcal{M}_{k}$ is dense at 0 , by (E). Moreover, for $\varepsilon \in \cap_{k} \mathcal{M}_{k}$, the sequence $\left\{\nu_{k}(\varepsilon)\right\} \subset B$ is Cauchy in $E_{r}$. Since $\Phi(\cdot, \varepsilon)$ is continuous on $B \subset E_{r}$ and $\Phi\left(\nu_{k}(\varepsilon), \varepsilon\right) \rightarrow 0$ in $F_{\rho}$, the result follows since $E_{r}$ is complete.

\section{O Small Divisors and Hypothesis (E)}

In this section we examine hypotheses (D) and (E) of the Nash-Moser theorem in the context of the standing-wave problem. As in Remark N.1, suppose that the spaces $E_{s}$ and $F_{s}$ are closed subspaces of the Sobolev space $H_{\text {ung }}^{s}$. Suppose also that in hypotheses (B) of Appendix $\mathrm{N}$, the linear operator $\Lambda(u, \varepsilon)$ is a perturbation of the operator $L(u, \varepsilon)$ which acts on elements of $E_{s}$ as a Fourier multiplication operator of the form

$$
(L(u, \varepsilon) v)_{m n}^{\wedge}=\left(-n^{2}+\left(1+\beta^{(0)}(u, \varepsilon)|m|-\varepsilon^{4} \kappa^{(1)}(u, \varepsilon)\right) \hat{v}_{m n}\right.
$$

equivalent to the pseudo-differential operator, with constant coefficients depending on $(u, \varepsilon)$,

$$
\partial_{\tau \tau}-\left(1+\beta^{(0)}(u, \varepsilon)\right) \mathcal{H} \partial_{\xi}-\varepsilon^{4} \kappa^{(1)}(u, \varepsilon) .
$$

Here (with $\kappa^{(0)}=\varepsilon^{4} \kappa^{(1)}$ in Lemma 7.6 ) and $N_{0} \geq 4$,

$$
\begin{aligned}
\beta^{(0)}(u, \varepsilon) & =\frac{\varepsilon^{2}}{4}+\breve{\beta} \varepsilon^{3}+\varepsilon^{4} \tilde{\beta}(u, \varepsilon), \\
\kappa^{(1)}(u, \varepsilon) & =\frac{1}{8}+\breve{\kappa} \varepsilon+\varepsilon \tilde{\kappa}(u, \varepsilon),
\end{aligned}
$$

where $\breve{\beta}$ and $\breve{\kappa}$ are real-valued functions of $\varepsilon$, and $\tilde{\beta}$ and $\tilde{\kappa}$ are smooth functions on $B \times\left[0, \varepsilon_{0}\right] \subset E_{r} \times \mathbb{R}$. In hypothesis $(\mathrm{D}), u=\nu(\varepsilon), \varepsilon \in \mathcal{E}$, and the coefficients (O.1) depend only on $\varepsilon \in \mathcal{E}$. In (E), a sequence of functions $\left\{\nu_{k}\right\}$ is defined 
recursively and the coefficients in (O.1) at each step depend on $\varepsilon \in \mathcal{E}\left(\nu_{k-1}\right)$. To understand the invertibility of $\Lambda(\nu(\varepsilon), \varepsilon)$ in (D) or (E) we first study the invertibility of $L(\nu(\varepsilon), \varepsilon)$, which means estimating solutions $\left\{\hat{u}_{m n}\right\}$ of the equation

$$
\left(-n^{2}+\left(1+\beta^{(0)}(\varepsilon)\right)|m|-\varepsilon^{4} \kappa^{(1)}(\varepsilon)\right) \hat{u}_{m n}=\hat{g}_{m n},
$$

where $\left\{\hat{g}_{m n}\right\}$ is given and $\left|\beta^{(0)}(\varepsilon)\right|<1$ for $\varepsilon_{0}$ sufficiently small. It suffices, after dividing by $1+\beta^{(0)}(\varepsilon)$, to estimate solutions of the equation

$$
\left(\left(1-\lambda(\varepsilon)^{2}\right) n^{2}-|m|+\varepsilon^{4} \kappa(\varepsilon)\right) u_{m n}=g_{m n}, \quad m \neq n^{2},
$$

where now

$$
\lambda(\varepsilon)=\sqrt{\frac{\beta^{(0)}(\varepsilon)}{1+\beta^{(0)}(\varepsilon)}} \text { and } \kappa(\varepsilon)=\frac{\kappa^{(1)}(\varepsilon)}{1+\beta^{(0)}(\varepsilon)} .
$$

Our first objective is to estimate the measure of the set of $\varepsilon \in\left[0, \varepsilon_{0}\right]$ for which

$$
\left|\left(1-\lambda(\varepsilon)^{2}\right) n^{2}-m+\varepsilon^{4} \kappa(\varepsilon)\right| \geq\left(2 n^{2}\right)^{-1} \text { for all } m, n \in \mathbb{N} \text { with } m \neq n^{2} .
$$

Note that this set depends on the function $\nu$.

\section{O.1 Small divisors}

Suppose that $\nu:\left[0, \varepsilon_{0}\right] \rightarrow E_{r}$ is Lipschitz continuous. Then, with $u=\nu(\varepsilon)$ in $(\mathrm{O} .1)$, the function $\lambda$ defined on $\left[0, \varepsilon_{0}\right]$ by $(\mathrm{O} .2)$ can be written in the form

$$
\lambda(\varepsilon)=\varepsilon\left(\frac{1}{2}+\varepsilon \psi(\varepsilon)+\varepsilon^{2} \eta(\varepsilon)\right),
$$

for some fixed function $\psi \in C^{1}(\mathbb{R})$ where

$$
\begin{aligned}
|\eta(\varepsilon)|+|\kappa(\varepsilon)| & \leq K, \\
\left|\eta\left(\varepsilon_{1}\right)-\eta\left(\varepsilon_{2}\right)\right|+\left|\kappa\left(\varepsilon_{1}\right)-\kappa\left(\varepsilon_{2}\right)\right| & \leq K\left|\varepsilon_{1}-\varepsilon_{2}\right|, \quad \varepsilon, \varepsilon_{1}, \varepsilon_{2} \in\left[0, \varepsilon_{0}\right],
\end{aligned}
$$

$\varepsilon_{0} \leq(1 / 2 K)^{1 / 4}$ and $K \in \mathbb{R}$ is such that $|\psi|+\left|\psi^{\prime}\right| \leq K$. To estimate the measure of the set of $\varepsilon \in\left[0, \varepsilon_{0}\right]$ for which (O.3) holds note that if $m>n^{2}$,

$$
\left|\left(1-\lambda(\varepsilon)^{2}\right) n^{2}-m+\varepsilon^{4} \kappa(\varepsilon)\right| \geq 1-\varepsilon^{4} \kappa(\varepsilon)>1 /\left(2 n^{2}\right)
$$

since $\varepsilon<\varepsilon_{0} \leq(1 / 2 K)^{1 / 4}$. Therefore it suffices to replace $m$ by $n^{2}-m$ and to study instead the set

$$
\left\{\varepsilon \in\left[0, \varepsilon_{0}\right]:|d(m, n, \varepsilon)| \geq(2 n)^{-4} \text { for all } m, n \in \mathbb{N}\right\}
$$

where

$$
d(m, n, \varepsilon)=\lambda(\varepsilon)^{2}-\frac{m}{n^{2}}-\frac{\varepsilon^{4} \kappa(\varepsilon)}{n^{2}}, \quad m, n \in \mathbb{N}, \quad \varepsilon \in \mathcal{E}_{0}
$$


Lemma O.1. If (O.5) is satisfied, $\varepsilon_{0} \in(0,1)$ can be chosen (independent of $\psi$, $\kappa$ and $\eta)$ such that

$$
\begin{gathered}
\frac{1}{4}\left(\varepsilon_{1}-\varepsilon_{2}\right) \leq \lambda\left(\varepsilon_{1}\right)-\lambda\left(\varepsilon_{2}\right) \leq \varepsilon_{1}-\varepsilon_{2}, \\
\varepsilon_{1}\left(\varepsilon_{1}-\varepsilon_{2}\right) \geq d\left(m, n, \varepsilon_{1}\right)-d\left(m, n, \varepsilon_{2}\right) \geq \frac{1}{3}\left(\varepsilon_{1}-\varepsilon_{2}\right) \varepsilon_{2},
\end{gathered}
$$

for all $m, n \in \mathbb{N}$, and for all $\varepsilon_{1}, \varepsilon_{2} \in\left[0, \varepsilon_{0}\right]$ with $\varepsilon_{1} \geq \varepsilon_{2}$.

Proof. That $\varepsilon_{0}$ can be chosen in this way is immediate from (O.5).

Since $d(m, n, 0)=-m / n^{2}<0$, Lemma O.1 shows that, for $(m, n)$ fixed,

$$
\left\{\varepsilon:|d(m, n, \varepsilon)|<\frac{1}{2 n^{4}}, 0<\varepsilon<\varepsilon_{0}\right\}=\left(\varepsilon^{-}(m, n), \varepsilon^{+}(m, n)\right) \cap\left(0, \varepsilon_{0}\right),
$$

where $\varepsilon^{ \pm}(m, n)$ are the roots of the equations

$$
d\left(m, n, \varepsilon^{ \pm}\right)= \pm \frac{1}{2 n^{4}} .
$$

Let $I(m, n)=\left(\varepsilon^{-}(m, n), \varepsilon^{+}(m, n)\right)$.

Lemma O.2. If $\left[\varepsilon^{-}(m, n), \varepsilon^{+}(m, n)\right] \cap[0, r] \neq \emptyset$ and $r \leq \varepsilon_{0}$, then

$$
\begin{gathered}
\frac{\sqrt{m}}{n} \leq \varepsilon^{-}(m, n) \leq \varepsilon^{+}(m, n) \leq 3 \frac{\sqrt{m}}{n}, \\
\frac{1}{3} \frac{1}{n^{3} \sqrt{m}} \leq \varepsilon^{+}(m, n)-\varepsilon^{-}(m, n) \leq \frac{3}{n^{3} \sqrt{m}}, \\
m \leq r^{2} n^{2} \text { which implies that } n \geq \frac{1}{r}>1 .
\end{gathered}
$$

Proof. From Lemma O.1, the graph $\left\{(\varepsilon, d(m, n, \varepsilon)): \varepsilon \in\left[0, \varepsilon_{0}\right]\right\}$ lies between two parabolae, $\left\{\left(\varepsilon, \varepsilon^{2} / 6-m / n^{2}\right): \varepsilon \in\left[0, \varepsilon_{0}\right]\right\}$ and $\left\{\left(\varepsilon, \varepsilon^{2} / 2-m / n^{2}\right): \varepsilon \in\left[0, \varepsilon_{0}\right]\right\}$. Now $\left[\varepsilon^{-}, \varepsilon^{+}\right] \cap[0, r] \neq \emptyset$ implies that $r \geq \varepsilon^{-} \geq \sqrt{m} / n$ and that (O.11) holds. It also implies (O.9), and (O.7), (O.8) and (O.9) lead to (O.10).

Corollary O.3. The set $\mathcal{E}(\nu)$ of $\varepsilon$ for which (O.3) holds is dense at 0 because meas $\{\varepsilon \in[0, r]:(\mathrm{O} .3)$ is false $\} \leq 6 r^{2}(1+r)$. 
Proof. Simply note from (O.10) and (O.11) that

$$
\begin{aligned}
\operatorname{meas}\{\varepsilon \in[0, r] & :(\mathrm{O} .3) \text { is false }\} \\
& \leq \sum_{\substack{m \neq n^{2} \\
I(m, n) \cap[0, r] \neq \emptyset}} \text { meas } I(m, n) \\
& \leq \sum_{m \leq n^{2} r^{2}} \frac{3}{n^{3} \sqrt{m}} \leq \sum_{n \geq 1 / r} \frac{3}{n^{3}} \sum_{m=1}^{n^{2} r^{2}} \frac{1}{\sqrt{m}} \\
& \leq \sum_{n \geq 1 / r} \frac{3}{n^{3}}\left(\int_{0}^{n^{2} r^{2}} \frac{d a}{\sqrt{s}}\right) \leq \sum_{n \geq 1 / r} \frac{6 r}{n^{2}} \\
& \leq 6 r\left(r^{2}+\int_{1 / r}^{\infty} \frac{d s}{s^{2}}\right)=6 r^{2}(1+r) .
\end{aligned}
$$

In the abstract setting of Theorem N.2 suppose that

$$
\left.\begin{array}{c}
\text { (N.1h) in hypothesis (D) is satisfied when } \\
\mathcal{E}(\nu)=\{\varepsilon \in \mathcal{E}: \varepsilon \text { satisfies (O.3) }
\end{array}\right\} .
$$

Remark. Corollary O.3 shows that if (O.12) holds then hypothesis (D) holds. That (O.12) holds in the context of the standing-wave equation (4.10) with $\Lambda$ given by (6.3) is proved in Theorem 8.5.

With the definition of $\mathcal{E}(\nu)$ in (O.12), hypothesis (E) in Appendix $\mathrm{N}$ can be verified using similar ideas to those in the proofs of Lemmas O.1, O.2 and Corollary O.3. But to do so we need a technical result on how Lipschitz continuous functions on closed subsets of $\left[0, \varepsilon_{0}\right]$ can be extended to $\left[0, \varepsilon_{0}\right]$.

\section{O.2 An extension}

Consider a collection of functions $\chi_{j}: \mathcal{E}_{j} \mapsto \mathbb{R}, j \in\{0, \cdots, k\}$, where $\mathcal{E}_{j}$ is compact, such that $\mathcal{E}_{0}=\left[0, \varepsilon_{0}\right], \quad \mathcal{E}_{j+1} \subset \mathcal{E}_{j}$, and suppose that

$$
\begin{aligned}
\left|\chi_{j}(x)-\chi_{j}(y)\right| & \leq K|x-y| \text { for } x, y \in \mathcal{E}_{j}, \quad j \in\{0, \cdots, k\}, \\
\left|\chi_{j}(x)-\chi_{j-1}(x)\right| & \leq \delta_{j} \text { for } x \in \mathcal{E}_{j}, j \in\{1, \cdots, k\} .
\end{aligned}
$$

Lemma O.4. Under the above assumptions there are functions $\bar{\chi}_{j}: \mathcal{E}_{0} \mapsto \mathbb{R}$, such that

$$
\begin{aligned}
\bar{\chi}_{j}(x) & =\chi_{j}(x) \text { for } x \in \mathcal{E}_{j}, \quad j \in\{0, \cdots, k\}, \\
\left|\bar{\chi}_{j}(x)-\bar{\chi}_{j}(y)\right| & \leq K|x-y| \text { for } x, y \in \mathcal{E}_{0}, \quad j \in\{0, \cdots, k\}, \\
\left|\bar{\chi}_{j}(x)-\bar{\chi}_{j-1}(x)\right| & \leq \delta_{j} \text { for } x \in \mathcal{E}_{0}, \quad j \in\{1, \cdots, k\} .
\end{aligned}
$$


Proof. The proof depends on the following simple construction. For any interval $[\alpha, \beta]$ and for any function $\chi:[\alpha, \beta] \mapsto \mathbb{R}$ which satisfies

$$
|\chi(x)-\chi(y)| \leq K|x-y| \text { for each } x \in[\alpha, \beta],
$$

let $g_{\alpha}, g_{\beta} \in \mathbb{R}$ be such that

$$
\left|g_{\alpha}-\chi(\alpha)\right| \leq \delta, \quad\left|g_{\beta}-\chi(\beta)\right| \leq \delta, \quad\left|g_{\alpha}-g_{\beta}\right| \leq K(\beta-\alpha) .
$$

Let

$$
h(x)=g_{\alpha}+\left(\frac{g_{\beta}-g_{\alpha}}{\beta-\alpha}\right)(x-\alpha) \text { and } k(x)=\min \{h(x), \chi(x)+\delta\} .
$$

It is now easy to see that the function

$$
g(x)=\max \{k(x), \chi(x)-\delta\}
$$

has the following properties:

$$
\begin{gathered}
|g(x)-g(y)| \leq K|x-y| \text { for each } x, y \in[\alpha, \beta], \\
|g(x)-\chi(x)| \leq \delta \text { for each } x \in[\alpha, \beta], \\
g(\alpha)=g_{\alpha}, \quad g(\beta)=g_{\beta} .
\end{gathered}
$$

Now suppose that we have defined the functions $\bar{\chi}_{i}$ for $i \leq j<k$. Set $\bar{\chi}_{j+1}(x)=$ $\chi_{j+1}(x)$ for $x \in \mathcal{E}_{j+1}$. Next note that the set $\mathcal{E}_{0} \backslash \mathcal{E}_{j+1}$ is a union of a countable family of disjoint open intervals $(\alpha, \beta)$ and, possibly, one or both of the halfopen intervals $[0, \beta)$ and $\left(\alpha, \varepsilon_{0}\right]$ where $\alpha, \beta \in \mathcal{E}_{j+1}$. In the above construction let $(\alpha, \beta)$ be one of these open intervals. Set $\chi(x)=\bar{\chi}_{j}(x)$ for $x \in[\alpha, \beta]$, $g_{\alpha}=\bar{\chi}_{j+1}(\alpha)$ and $g_{\beta}=\bar{\chi}_{j+1}(\beta)$. Then we can define $\bar{\chi}_{j+1}(x)=g(x)$ for $x \in[\alpha, \beta]$, where $g$ is given above with $\delta=\delta_{j+1}$. To extend $\chi_{j+1}$ to an interval of the form $[0, \beta)$ where $\beta \in \mathcal{E}_{j+1}$ but $0 \notin \mathcal{E}_{j+1}$ let

$$
\bar{\chi}_{j+1}(x)=\bar{\chi}_{j}(x)+\chi_{j+1}(\beta)-\bar{\chi}_{j}(\beta), \quad x \in[0, \beta) .
$$

Since $\left|\chi_{j+1}(\beta)-\bar{\chi}_{j}(\beta)\right|<\delta_{j+1}$, this extension has the required properties. The extension to intervals of the form $\left(\alpha, \varepsilon_{0}\right]$ is similar.

\section{O.3 Hypothesis (E)}

Suppose that (O.12) holds, $\nu_{0}: \mathcal{E}_{0} \rightarrow B \cap E_{r+l}$ and mappings $\nu_{k}: \cap_{i=0}^{k-1} \mathcal{E}\left(\nu_{i}\right) \rightarrow$ $B \cap E_{r+l}$ satisfy, for a constant $C$ independent of $k \in \mathbb{N}$ sufficiently large,

$$
\begin{gathered}
\left\|\nu_{k}\left(\varepsilon_{1}\right)-\nu_{k}\left(\varepsilon_{2}\right)\right\|_{r} \leq C\left|\varepsilon_{1}-\varepsilon_{2}\right|, \quad \varepsilon_{1}, \varepsilon_{2} \in \cap_{j=0}^{k-1} \mathcal{E}\left(\nu_{j}\right), \\
\left\|\nu_{k+1}(\varepsilon)-\nu_{k}(\varepsilon)\right\|_{r} \leq \frac{1}{2^{k}}, \quad \varepsilon \in \cap_{j=0}^{k} \mathcal{E}\left(\nu_{j}\right) .
\end{gathered}
$$

Thanks to Corollary O.3, for each $k, \mathcal{E}\left(\nu_{k}\right)$ is dense at zero and so also is the intersection of a finite collection of sets $\mathcal{E}\left(\nu_{k}\right)$. Our goal is to show that the 
intersection of all the sets $\mathcal{E}\left(\nu_{k}\right)$ is also dense at 0 . For $k \in \mathbb{N}$ the coefficients $\hat{\beta}_{k}(\varepsilon):=\hat{\beta}\left(\nu_{k}(\varepsilon), \varepsilon\right)$ and $\hat{\kappa}_{k}(\varepsilon):=\hat{\kappa}\left(\nu_{k}(\varepsilon), \varepsilon\right)$ are defined by (O.1) on the set $\mathcal{E}\left(\nu_{k-1}\right)$, and the corresponding $\lambda=\lambda_{k}, \eta=\eta_{k}$ and $\kappa=\kappa_{k}$ (defined in terms of $\hat{\beta}_{k}$ and $\left.\hat{\kappa}_{k}\right)$ satisfy, for $\varepsilon, \varepsilon_{1}, \varepsilon_{2} \in \mathcal{E}\left(\nu_{k-1}\right)$, for $k$ sufficiently large,

$$
\begin{aligned}
\left|\eta_{k}(\varepsilon)\right|+\left|\kappa_{k}(\varepsilon)\right| & \leq K, \\
\left|\eta_{k}\left(\varepsilon_{1}\right)-\eta_{k}\left(\varepsilon_{2}\right)\right|+\left|\kappa_{k}\left(\varepsilon_{1}\right)-\kappa_{k}\left(\varepsilon_{2}\right)\right| & \leq K\left|\varepsilon_{1}-\varepsilon_{2}\right|, \\
\left|\eta_{k}(\varepsilon)-\eta_{k-1}(\varepsilon)\right|+\left|\kappa_{k}(\varepsilon)-\kappa_{k-1}(\varepsilon)\right| & \leq \frac{1}{2^{k}},
\end{aligned}
$$

with $K$ independent of $k$. Our analysis of hypothesis (E) is based on the characterisation of $\mathcal{E}\left(\nu_{k}\right)$ in (O.12). We will show that the set of $\varepsilon>0$ for which (O.3), with $\lambda=\lambda_{k}$ (defined in terms of $\psi$ and $\eta_{k}$ by (O.4)) and $\kappa=\kappa_{k}$ holds for all $k \geq 0$ is dense at 0 . We do so by estimating from above the size of the set where (O.3) fails for at least one $k$, for some $\varepsilon \in \cap_{j=0}^{k-1} \mathcal{E}\left(\nu_{j}\right)$. It therefore suffices to find such an upper estimate when $\kappa_{k}$ and $\eta_{k}$ are replaced by their extensions (see Lemma O.5) as functions which satisfy (O.13) on $\mathcal{E}_{0}$. Suppose therefore that $(\mathrm{O} .13)$ hold on $\mathcal{E}_{0}$ for all $k$. By $(\mathrm{O} .13 \mathrm{c})$ there exists $\kappa_{\infty}$ and $\eta_{\infty}$ which satisfy (O.13a) and (O.13b), and

$$
\left|\eta_{k}(\varepsilon)-\eta_{\infty}(\varepsilon)\right|+\left|\kappa_{k}(\varepsilon)-\kappa_{\infty}(\varepsilon)\right| \leq \frac{1}{2^{k}}, \quad \varepsilon \in \mathcal{E}_{0} .
$$

For $k \in\{\infty\} \cup \mathbb{N}_{0}$ let

$$
d_{k}(m, n, \varepsilon)=\lambda_{k}(\varepsilon)^{2}-\frac{m}{n^{2}}-\frac{\varepsilon^{4} \kappa_{k}(\varepsilon)}{n^{2}}, m, n \in \mathbb{N}, \quad \varepsilon \in \mathcal{E}_{0} .
$$

With subscripts $k$ denoting $k$-dependence, let $I_{k}(m, n)=\left(\varepsilon_{k}^{-}(m, n), \varepsilon_{k}^{+}(m, n)\right)$.

Lemma O.5. If $I_{j}(m, n) \cap[0, r] \neq \emptyset$ and $r \in\left(0, \varepsilon_{0}\right)$, then

$$
\left|\varepsilon_{j+1}^{ \pm}(m, n)-\varepsilon_{j}^{ \pm}(m, n)\right| \leq \frac{3 A}{2^{j+1}} \frac{m^{3 / 2}}{n^{3}} \quad \text { where } A=2 \times 3^{4} .
$$

Proof. To begin we estimate $\left|\varepsilon_{j+1}^{ \pm}-\varepsilon_{j}^{ \pm}\right|$. Since $d_{j+1}\left(m, n, \varepsilon_{j+1}^{ \pm}\right)=d_{j}\left(m, n, \varepsilon_{j}^{ \pm}\right)$,

$$
\left|d_{j+1}\left(m, n, \varepsilon_{j+1}^{ \pm}\right)-d_{j+1}\left(m, n, \varepsilon_{j}^{ \pm}\right)\right|=\left|d_{j+1}\left(m, n, \varepsilon_{j}^{ \pm}\right)-d_{j}\left(m, n, \varepsilon_{j}^{ \pm}\right)\right| .
$$

Hence, by (O.7) and (O.9),

$$
\frac{\sqrt{m}}{3 n}\left|\varepsilon_{j+1}^{ \pm}-\varepsilon_{j}^{ \pm}\right| \leq\left|d_{j+1}\left(m, n, \varepsilon_{j}^{ \pm}\right)-d_{j}\left(m, n, \varepsilon_{j}^{ \pm}\right)\right| .
$$

On the other hand,

$$
\begin{aligned}
\mid d_{j+1}( & \left.m, n, \varepsilon_{j}^{ \pm}\right)-d_{j}\left(m, n, \varepsilon_{j}^{ \pm}\right) \mid \\
= & \mid \varepsilon_{j}^{ \pm}\left(\eta_{j+1}\left(\varepsilon_{j}^{ \pm}\right)-\eta_{j}\left(\varepsilon_{j}^{ \pm}\right)\right)\left(\lambda_{j+1}\left(\varepsilon_{j}^{ \pm}\right)+\lambda_{j}\left(\varepsilon_{j}^{ \pm}\right)\right) \\
& -\frac{\varepsilon_{j}^{ \pm}}{n^{2}}\left(\kappa_{j+1}\left(\varepsilon_{j}^{ \pm}\right)-\kappa_{j}\left(\varepsilon_{j}^{ \pm}\right)\right) \mid .
\end{aligned}
$$


Now (O.6) and (O.9) imply that $\left|\lambda_{j+1}\left(\varepsilon_{j}^{ \pm}\right)\right|+\left|\lambda_{j}\left(\varepsilon_{j}^{ \pm}\right)\right| \leq 2 \varepsilon_{j}^{ \pm} \leq 6 \sqrt{m} / n$ and so

$$
\begin{aligned}
\mid d_{j+1}\left(m, n, \varepsilon_{j}^{ \pm}\right) & -d_{j}\left(m, n, \varepsilon_{j}^{ \pm}\right) \mid \\
& \leq\left(2 \times 3^{4}\right) \frac{m^{2}}{n^{4}}\left(\left|\eta_{j+1}\left(\varepsilon_{j}^{ \pm}\right)-\eta_{j}\left(\varepsilon_{j}^{ \pm}\right)\right|+\left|\kappa_{j+1}\left(\varepsilon_{j}^{ \pm}\right)-\kappa_{j}\left(\varepsilon_{j}^{ \pm}\right)\right|\right) \\
& \leq \frac{A m^{2}}{2^{j+1} n^{4}}, \text { by }(\mathrm{O} .13 \mathrm{c}) \text { where } A=2 \times 3^{4} .
\end{aligned}
$$

After a substitution into (O.15), this observation and (O.11) shows (O.14).

It follows from the triangle inequality that for all $k$,

$$
\left|\varepsilon_{\infty}^{ \pm}(m, n)-\varepsilon_{k}^{ \pm}(m, n)\right| \leq \frac{3 A}{2^{k}} \frac{m^{3 / 2}}{n^{3}} .
$$

Lemma O.6. If $A m^{2} / 2^{k} \leq 1$, then, $I_{k}(m, n) \subset J_{\infty}(m, n)$, where $J_{\infty}(m, n)$ is an interval with the same centre as $I_{\infty}(m, n)$ and three times the length. Hence

$$
\operatorname{meas}\left(\bigcup_{\substack{k \in \mathbb{N} \\ m \leq n^{2} r^{2} \\ A m^{2} \leq 2^{k}}} I_{j}(m, n)\right) \leq 18 r^{2}(1+r) .
$$

Proof. That $I_{k}(m, n) \subset J_{\infty}(m, n)$ when $A m^{2} \leq 2^{k}$ is immediate from (O.10), with subscripts $k$ added, and (O.16). The result follows from Corollary O.3.

Lemma O.7. For an absolute constant,

$$
\operatorname{meas}\left(\bigcup_{\substack{k \in \mathbb{N} \\ m \leq r^{2} n^{2} \\ A m^{2} \geq 2^{k}}} I_{k}(m, n)\right) \leq \text { const. } r^{2} .
$$

Proof. Note from (O.10) and (O.11) that

$$
\begin{aligned}
\sum_{\substack{k \in \mathbb{N} \\
m \leq n^{2} r^{2} \\
A m^{2} \geq 2^{k}}} I_{k}(m, n) \leq \sum_{\substack{k \in \mathbb{N} \\
m \leq r^{2} n^{2} \\
A m^{2} \geq 2^{k}}} \frac{3}{n^{3} \sqrt{m}} \leq \sum_{\substack{k \in \mathbb{N} \\
\left\{n: A n^{4} r^{4} \geq 2^{k}\right\}}} \frac{6 r}{n^{2}} \\
\leq 12 A^{1 / 4} r^{2} \sum_{k \in \mathbb{N}} \frac{1}{2^{k / 4}}=\text { const. } r^{2} .
\end{aligned}
$$

Theorem O.8. The set $\cap_{k \in \mathbb{N}_{0}} \mathcal{E}_{k}$ is dense at zero. In other words (E) is satisfied.

Proof. This is immediate from the preceding two lemmas. 


\section{References}

[1] C. J. Amick and J. F. Toland, The semi-analytic theory of standing waves, Proc. Roy. Soc. Lond. A 411 (1987), 123-138.

[2] V. I. Arnol'd, Small denominators and problems of stability of motion in classical and celestial mechanics, Russian Math. Surv. 18 (6) (1963), 85-191.

[3] D. Bambusi and S. Paleari. Families of periodic soluitions of resonant PDEs, J. Nonlinear Sci. 11 (2001), 69-87.

[4] T. B. Benjamin and P. J. Olver, Hamiltonian structures, symmetries and conservation laws for water waves, J. Fluid Mech. 125 (1982), 137-187.

[5] M. Berti and P. Bolle, Periodic solutions of nonlinear wave equations with general nonlinearities, Comm. Math. Phys. 243 (2) (2003), 315-328.

[6] Multiplicity of periodic solutions of nonlinear wave equations, Nonlinear Analysis TMA, 56 (7) (2004), 1011-1046.

[7] J. Boussinesq, Essai sur la théorie des eaux courantes, Mémoires présentés par divers savants à l'Académie des Sciences, Paris 23 (1), (1877), 1-660

[8] H. Brezis, Periodic solutions of nonlinear vibrating strings and duality principles, Bull. A.M.S. 8 (3), (1983), 409-426.

[9] W. Craig and C. E. Wayne, Newton's method and periodic solutions of nonlinear wave equations, Comm. Pure Appl. Math. 46 (1993), 1409-1498.

[10] A. D. Craik, The origins of water wave theory. Annual review of Fluid Mechanics Vol. 36,(2004) 1-28.

[11] A. I. Dyachenko, E. A. Kuznetsov, M. D. Spector, and V. E. Zakharov, Analytic description of the free surface dynamics of an ideal fluid (canonical formalism and conformal mapping), Physics Letters A 221 (1996), 73-79.

[12] L. Hörmander, The boundary problems of physical geodesy, Arch. Rational Mech. Anal. 62 (1976), 1-52.

[13] G. Iooss, The semi-analytic theory of standing waves, for several dominant modes, Proc. Roy. Soc. Lond. A455 (1999), 3513-3526.

[14] 4 (2002), 155-185.

[15] G. Iooss, P Plotnikov and J. Toland, Standing waves on infinite depth, Comptes Rendus Acad. Sci. Paris, 338 (5), (2004), 425-431.

[16] G. Iooss and J. F. Toland, Riemann-Hilbert and variational structure for standing waves. Preprint, to appear. 
[17] T. Kato. Perturbation Theory for Linear Operators. Springer-Verlag, NewYork 1966.

[18] C. B. Morrey. Multiple Integrals in the Calculus of Variations. SpringerVerlag, Berlin, 1966.

[19] J. Moser, A rapidly convergent iteration method and non-linear partial differential equations I \& II, Ann. Scuola Norm. Sup. Pisa, Sci. Fiz. Mat., III. Ser. 20, 265-315 \& 499-535 (1966).

[20] M. Okamura, Standing waves of large amplitude in deep water, Wave Motion, 37 (2), (2003), 17-182.

[21] G. W. Penney and A. T. Price, Finite periodic stationary gravity waves in a perfect fluid, Phil. Trans. Roy. Soc. A 244 (1952), 254-284.

[22] P. I. Plotnikov and J. F. Toland, Nash-Moser theory for standing waves, Arch. Rational Mech. Anal. 159 (2001), 1-83.

[23] S. D. Poisson, Mémoire sur la théorie des ondes (1816). Mém. Acad. R. Sci. Inst. France, 2nd Series 1:70-186, 1818.

[24] P. S. Laplace, Suite des recherches sur plusieurs points du système du monde. (XXV-XXVII). Mém. présentés par divers savants à l'Acad. des Sciences, Paris, Sur les ondes 542-552.

[25] P. H. Rabinowitz, Free vibrations for a semilinear wave equation, Comm. Pure Appl. Math. 31 (1978), 31-68.

[26] Lord Rayleigh, Deep water waves, progressive or stationary, to the third order of approximation, Proc. R. Soc. London A 91 (1915), 345-353.

[27] L. W. Schwartz and A. K. Whitney, A semi-analytic solution for nonlinear standing waves in deep water, J. Fluid Mechanics 107 (1981), 147-171.

[28] Ya. I. Sekerzh-Zenkovich, On the theory of standing waves of finite amplitude, Doklady AN USSR 58 (1947), 551-553.

[29] G. G. Stokes. On the theory of oscillatory waves, Trans. Camb. Phil. Soc.,8, (1847), 441-455.

[30] Considerations relative to the greatest height of oscillatory irrotational waves which can be propagated without change of form, Mathematical and Physical Papers, vol. I, Cambridge, 1880, pp. 225-228.

[31] I. Tadjbaksh and J. B. Keller, Standing surface waves of finite amplitude, J. Fluid Mech. 8 (1960), 442-451.

[32] G. I. Taylor, An experimental study of standing waves, Proc. Roy. Soc. Lond. A. (1953), 44-59. 
[33] C. E. Wayne, Periodic and quasi-periodic solutions of nonlinear wave equations via KAM theory, Comm. Math. Phys. 127 (1990), 479-528.

[34] _ Periodic solutions of nonlinear partial differential equations, Notices of Amer. Math. Soc. (Sept., 1997), 895-901.

[35] J. Wehausen, Free-surface flows, in Research Frontiers in Fluid Dynamics, Ed. R. J. Seeger \& G. Temple, Interscience, New York, 1965.

[36] V. E. Zakharov, Stability of periodic waves of finite amplitude on the surface of deep fluid, J. Appl. Mech. Tech. Phys. 2, (1968), 190-194.

[37] A. Zygmund, Trigonometric Series I \& II. corrected reprint (1968) of 2nd. ed., Cambridge University Press, Cambridge, 1959. 